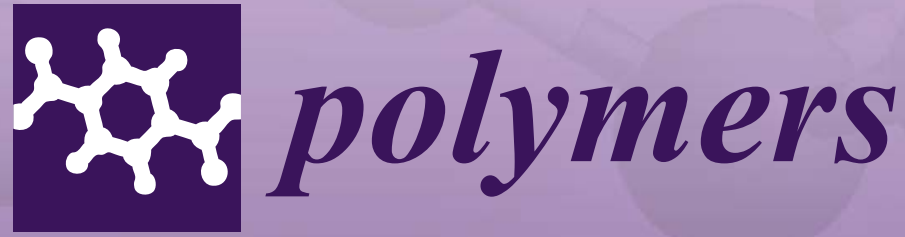

\title{
Siloxane-Based Polymers
}

Edited by Ignazio Blanco Printed Edition of the Special Issue Published in Polymers 
Siloxane-Based Polymers 



\section{Siloxane-Based Polymers}

Special Issue Editor

Ignazio Blanco

MDPI • Basel • Beijing • Wuhan • Barcelona • Belgrade

\section{MDPI}


Special Issue Editor

Ignazio Blanco

University of Catania

Italy

\section{Editorial Office}

MDPI

St. Alban-Anlage 66

Basel, Switzerland

This is a reprint of articles from the Special Issue published online in the open access journal Polymers (ISSN 2073-4360) from 2017 to 2018 (available at: http:/ / www.mdpi.com/journal/polymers/special_ issues/siloxane_based_polymers)

For citation purposes, cite each article independently as indicated on the article page online and as indicated below:

LastName, A.A.; LastName, B.B.; LastName, C.C. Article Title. Journal Name Year, Article Number, Page Range.

\section{ISBN 978-3-03897-125-2 (Pbk)}

ISBN 978-3-03897-126-9 (PDF)

Cover image courtesy of Ignazio Blanco.

Articles in this volume are Open Access and distributed under the Creative Commons Attribution (CC BY) license, which allows users to download, copy and build upon published articles even for commercial purposes, as long as the author and publisher are properly credited, which ensures maximum dissemination and a wider impact of our publications. The book taken as a whole is (C) 2018 MDPI, Basel, Switzerland, distributed under the terms and conditions of the Creative Commons license CC BY-NC-ND (http:/ / creativecommons.org/licenses/by-nc-nd/4.0/). 


\section{Contents}

About the Special Issue Editor $\ldots \ldots \ldots \ldots \ldots \ldots \ldots \ldots$ vii

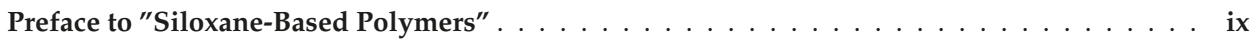

Ignazio Blanco

Polysiloxanes in Theranostics and Drug Delivery: A Review

Reprinted from: Polymers 2018, 10, 755, doi: 10.3390/polym10070755 . . . . . . . . . . 1

José González-Rivera, Rossella Iglio, Giuseppe Barillaro, Celia Duce and Maria Rosaria Tinè Structural and Thermoanalytical Characterization of 3D Porous PDMS Foam Materials: The Effect of Impurities Derived from a Sugar Templating Process

Reprinted from: Polymers 2018, 10, 616, doi: 10.3390/polym10060616 . . . . . . . . . . . . .

Michelina Catauro, Elisabetta Tranquillo, Roberta Risoluti and Stefano Vecchio Ciprioti

Sol-Gel Synthesis, Spectroscopic and Thermal Behavior Study of $\mathrm{SiO}_{2} /$ PEG Composites

Containing Different Amount of Chlorogenic Acid

Reprinted from: Polymers 2018, 10, 682, doi: 10.3390/polym10060682 . . . . . . . . . . . 25

Jun-Min Kim, Changkyun Im and Woo Ram Lee

Plateau-Shaped Flexible Polymer Microelectrode Array for Neural Recording

Reprinted from: Polymers 2017, 9, 690, doi: 10.3390/polym9120690 _ . . . . . . . . . . . . . 37

Xin Chen, Yancong Feng, Xiao Wang, En Li, Yao Wang, Lingling Shui, Hao Li, Nan Li and Guofu Zhou

Quartz Microcrystal-Hybridized Organosilicone Encapsulant with Enhanced Optical and Thermal Performances

Reprinted from: Polymers 2018, 10, 84, doi: 10.3390/polym10010084 . . . . . . . . . . . . .

Iryna Protsak, Evgeniy Pakhlov, Valentyn Tertykh, Zi-Chun Le and Wen Dong

A New Route for Preparation of Hydrophobic Silica Nanoparticles Using a Mixture of Poly(dimethylsiloxane) and Diethyl Carbonate

Reprinted from: Polymers 2018, 10, 116, doi: 10.3390/polym10020116 . . . . . . . . . . . 64

Peihong Li, Xiaoman Hou, Lijie Qu, Xueyan Dai and Chunling Zhang

PNIPAM-MAPOSS Hybrid Hydrogels with Excellent Swelling Behavior and Enhanced

Mechanical Performance: Preparation and Drug Release of 5-Fluorouracil

Reprinted from: Polymers 2018, 10, 137, doi: 10.3390/polym10020137 . . . . . . . . . . . 77

Yizhi Liu, Xiaorong Wu, Yi Sun and Weili Xie

POSS Dental Nanocomposite Resin: Synthesis, Shrinkage, Double Bond Conversion, Hardness, and Resistance Properties

Reprinted from: Polymers 2018, 10, 369, doi: 10.3390/polym10040369

Jianhua Guo, Xuming Chen and Yong Zhang

Improving the Mechanical and Electrical Properties of Ceramizable Silicone Rubber/Halloysite

Composites and Their Ceramic Residues by Incorporation of Different Borates

Reprinted from: Polymers 2018, 10, 388, doi: 10.3390/polym10040388 . . . . . . . . . . . . . 100

Danuta Barnat-Hunek, Grzegorz Łagód, Stanisław Fic and Monika Jarosz-Hadam

Effect of Polysiloxanes on Roughness and Durability of Basalt Fibres-Reinforced

Cement Mortar

Reprinted from: Polymers 2018, 10, 420, doi: 10.3390/polym10040420 . . . . . . . . . . . . 112 
Guillaume Gillet, Fabrice Azemar, Fabienne Faÿ, Karine Réhel and Isabelle Linossier

Non-Leachable Hydrophilic Additives for Amphiphilic Coatings

Reprinted from: Polymers 2018, 10, 445, doi: 10.3390/polym10040445

Jerzy J. Chruściel, Marzena Fejdyś and Witold Fortuniak

Synthesis, Characterization and Microstructure of New Liquid Poly(methylhydrosiloxanes)

Containing Branching Units $\mathrm{SiO}_{4 / 2}$

Reprinted from: Polymers 2018, 10, 484, doi: 10.3390/polym10050484 . . . . . . . . . . . . . . . 144

Ning Liu, Jianyi Yu, Yaoyong Meng and Yuzhou Liu

Hyperbranched Polysiloxanes Based on Polyhedral Oligomeric Silsesquioxane Cages with

Ultra-High Molecular Weight and Structural Tuneability

Reprinted from: Polymers 2018, 10,496, doi: 10.3390/polym10050496 . . . . . . . . . . . . . . 162 


\section{About the Special Issue Editor}

Ignazio Blanco is the Associate Professor of "Fundamentals of Chemicals Technology" at the Department of Civil Engineering and Architecture of the University of Catania. Prof. Blanco research activities are focused on the following themes: synthesis and characterization of toughened thermoset blends; process technology of polymeric fiber-reinforced composites; comparative kinetic study of the thermal degradation of model polymers and nanocomposites; thermal characterization of films used in food packaging application; synthesis and characterization of POSS nanoparticles; life-time predictions of polymeric materials. He is a member of the Editorial Board of the Journal of Composites Science and of the Board of the Italian Interdivisional Calorimetry and Thermal Analysis Group and acts as a Guest Editor for the journals Polymers and Materials and as a reviewer for several international journals. 



\section{Preface to "Siloxane-Based Polymers"}

Since the birth of chemistry, inorganic and organic compounds have been considered two separate worlds owing to their completely different properties and structure. As a result of the scientific progress in this field, it is possible today to obtain hybrid inorganic-organic materials, displaying the properties of both types, which are different from those achievable by their simple blend at a macroscopic level. In this frame, the synthesis of siloxane-based polymers represents at the same time a challenge and a fascinating field of research and offers the opportunity of obtaining compounds with new unique properties. This book contains original research and review articles that report the synthesis and characterization of these hybrid materials and attest the enhancement of their chemical, physical, electrical, and mechanical properties with respect to polymer matrix composites and other traditional materials. Both well-established and new chemical processes contributing to the preparation of such materials are reported. Their applications, also discussed in articles included in this book, are widespread from the medical to the electronic field, including cultural heritage and buildings, demonstrating the versatility of polysiloxane-based materials.

Ignazio Blanco

Special Issue Editor 



\title{
Polysiloxanes in Theranostics and Drug Delivery: A Review
}

\author{
Ignazio Blanco ${ }^{1,2}$ \\ 1 Department of Civil Engineering and Architecture, University of Catania, Viale Andrea Doria 6, \\ 95125 Catania, Italy; iblanco@unict.it \\ 2 UdR-Catania Consorzio INSTM, Viale Andrea Doria 6, 95125 Catania, Italy
}

Received: 3 June 2018; Accepted: 5 July 2018; Published: 9 July 2018

\begin{abstract}
One of the historical problems of medicine is that often, diagnosis and therapy do not interface, at best. Moreover, especially in some areas, such as oncology, the stress for the organism during the two phases (diagnosis and therapy) can become excessive, and be fatal to the success of the treatment. The extraordinary progress of nanotechnology in the last 25 years has offered the opportunity to build a nanoplatform able to ferry drugs, and loads onto them both imaging and therapeutic functions, thus creating nanosystems capable of diagnosis, drug delivery, and monitoring of therapeutic response. The purpose of this unusual, and up to recent times, unimaginable, marriage between diagnosis and therapeutics is the reaching of protocols more specific to individuals. The dual use of particles/device lead to a personalized medicine. Due to their biocompatibility, versatility, physical and chemical resistance, and ability to be functionalized, silica nanoparticles and polysiloxanes are the heart and the shield of this nanoplatform, respectively. In this short review, I analyze the applications of these silicon-based materials in the field of controlled drug delivery.
\end{abstract}

Keywords: polysiloxanes; theranostics; drug delivery; nanomedicine; PDMS; silicon

\section{Introduction}

When we talk about silicon-based compounds, our mind flies towards the Californian valley, that has made the term silicon famous to non-chemistry people. However silicon, before becoming the most widely used element in the manufacture (sometimes at high temperature and pressure) of siloxane-based semiconductors, was the chemical base of the simplest siloxane, $\left(\mathrm{SiO}_{2}\right)_{n}$, that is possible to find in nature in many organisms, such as structured shells, spines, fibers, and granules in many protists, diatoms, sponges, mollusks, and higher plants [1,2]. By taking inspiration from these biologically nanoscale architectures, chemistry and chemical-engineering scientists proposed, in the last 50 years, the replacing of these materials for medical and pharmaceutical applications.

Today, polymers are probably the greater and most important category of organic compounds that have changed our lifestyle, but already since the 1970s, polysiloxanes, because of their intrinsic biological nature, were used in the medical field. Huge opportunities in the design, synthesis, and modification of their physical and chemical properties have made them the most rapidly growing group of polymers, having great importance and possibilities for applications in pharmacy, medicine, and cosmetology [3].

We are writing about a very delicate field of application, perhaps the most delicate, where the keyword is compatibility, in the most severe sense of the term. From the clinical point of view, biomaterial compatibility requires many factors, among which the most important are the absence of thrombogenic, toxic, allergic or inflammatory reactions; no destruction of formed elements; no change in plasma proteins or enzymes; no immunological reactions; no carcinogenic effects; and no deterioration of adjacent tissues [4]. Coupling the biocompatibility aspects, which were written above, 
to their versatility (depending on the size of the molecule and on the shape of the skeleton, they can be liquids of varied viscosity, resins of varied consistence, rubbers, or elastomers), polysiloxanes are confirmed today, more than 50 years from their first applications, as one of the most used polymer categories in a wide range of biomedical applications.

The unique properties of polysiloxanes are due to the presence of both polar inorganic backbone and organic nonpolar functional groups, and as reported in many literature references [5-8], this hybrid nature is reflected in a close relationship between the structure of the polymer molecule and its physicochemical properties. The five principal structures of polysiloxanes are linear, cyclic ring polymer, branched, crosslinked, and crosslinked easily transformed into a three-dimensional network [3]. For all types of structures of polysiloxanes, four types of monomers (units) are used: monofunctional $(\mathrm{M})$, difunctional $(\mathrm{D})$, trifunctional $(\mathrm{T})$, and tetrafunctional $(\mathrm{Q})$.

Professor Pieńkowska, in her interesting review about the Pharmaceutical Applications of Polysiloxanes [3], divided these materials in four groups: non-volatile silicone fluids (linear straight chain, e.g., linear polydimethylsiloxanes, PDMS) for pharmaceutical use as an active pharmaceutical ingredient (API), and excipient, or for cosmetic use; volatile silicone fluids (typically cyclic volatile methylsiloxane, VMS), for cosmetic use; silicone elastomers intended for medical use on skin (e.g., transdermal delivery system, patches, medical use); and silicone elastomers for scar and keloid treatment.

Polydimethylsiloxane (PDMS) is an important example of this class of polymers, and as a result of their physiological inertness, good blood compatibility, low toxicity, good thermal and oxidative stability, low modulus and antiadhesive properties, good solubility in common organic solvents, good film-forming ability, fair adhesion to various substrates, and excellent resistance to chemical and irradiation degradation, we find them in a field of applications so broad that it embraces all sectors of medicine [4]. The $\mathrm{Si}-\mathrm{O}$ backbone of this class of polymers, coupled with the organic nature of the functional groups, endows it with a variety of intriguing properties. The $\mathrm{Si}-\mathrm{O}$ bond length is $1.64 \AA$, significantly longer than that of the $\mathrm{C}-\mathrm{C}$ bond $(1.53 \AA)$, and the $\mathrm{Si}-\mathrm{O}-\mathrm{Si}$ bond angle is approximately $143^{\circ}$, much larger than the usual tetrahedral value $\left(110^{\circ}\right)$. All these aspects translate into low barrier to rotation, low rotation energy $(\sim 0)$ and a torsional potential significantly lower than that about $\mathrm{C}-\mathrm{C}$ bonds, thus making the PDMS chain one of the most flexible known [3,9]. Furthermore, the nonpolar organic substituents (e.g., methyl group), present shorter $\mathrm{Si}-\mathrm{C}$ bond, no steric hindrance, ease of reorientation, weak intermolecular forces, and opportunity to substitute other functional groups [3].

The long list of PDMS applications includes both its use as an active substance in medical devices (blood pumps, cardiac pacemaker leads, mammary prostheses, drainage implants in glaucoma, artificial skin, maxillofacial reconstruction, replacement esophagus, contact lenses, oxygenators, medical adhesives, finger joints, coatings for cochlear implants, catheters, drug delivery systems, and denture liners) [4], as well as excipient (skin adhesive patches, pressure-sensitive silicone adhesives, again controlled drug delivery systems, skin care emollients, ointments and lotions, and various auxiliary materials like contact lenses, various implants) [3]. This review aims to focus on one of the most widespread applications of this important class of polymers, drug delivery, by highlighting, where necessary, also, the possible improvements related to the interaction with the human body, because despite its bio-inertness, some problems can be recorded in this regard.

\section{Polysiloxanes in Theranostics and Drug Delivery}

\subsection{Brief Summary of the History of Polymer Applications in the Theranostic Field}

In the perspective of minimizing toxicity to the human body and increasing the drug efficiency, theranostics tries to perform diagnosis and therapy at the same time, identifying the individual tumor particles [10-12]. The key role is entrusted to the nanoparticles accompanied in the place of interest, inside the body, by an external magnetic field. 
Regarding the diagnosis, nanoparticles can be used as agents in magnetic resonance imaging (MRI), to increase the contrast of the image and allow better detection of tumors. As for the therapy, they are used for magnetic hyperthermia, a technique based on heating by applying a weak alternating magnetic field with an appropriate frequency. In this way, the cancer cells, being more sensitive to heat than healthy ones, are destroyed, once a local temperature between 41 and $46^{\circ} \mathrm{C}$ is reached [13].

In this regard, different nanoparticles have been developed in the field of theranostics $[14,15]$ aiming to exhibit long circulation in body fluids (ensuring greater accumulation in tumor tissues due to their active or passive targeting properties); to be rapidly eliminated through the renal route to ensure a sufficient difference in concentration between healthy and diseased zones; and to display therapeutic potential and contrast properties.

In both cases, multifunctional nanoparticles must be coated with a polymer, which possesses a series of particular characteristics, in order to protect the cores and to provide functionality (e.g., biological recognition functions, thermoresponsivity, and catalytic properties) [16]. Surface modification of nanoparticles with coupling agents can be employed to offer them better compatibility with dispersing media, to prevent nanoparticles from aggregation, as well as to render them with chemical reactivity [17-19]. This also avoids homogeneity and compatibility problems between the two phases, and thus, designing materials with well-adjusted properties makes their specific use possible.

3-Glycidoxypropyltrimethoxysilane (GLYMO), 3-aminopropyltriethoxysilane (APTES), and 3-methacryloxypropyltrimethoxysilane (MEMO) have been largely used as precursors for organic-inorganic hybrid sol-gel materials [14,15]. GLYMO, APTES, and MEMO have the ability to form, simultaneously, an inorganic $=\mathrm{SieOeSi}=$ network through hydrolysis and condensation reactions of alkoxy groups, and covalent network with polymer through the polymerization of the corresponding epoxy, amine, and acrylate groups [20]. Silane coupling agents, that form a durable bond between organic and inorganic materials, typically show two classes of functionality.

- Hydrolyzable group, typically alkoxy, acyloxy, halogen or amine. Following hydrolysis, a reactive silanol group is formed, which can condense with other silanol groups to form siloxane linkages. - Nonhydrolyzable organic radical that may possess a functionality that imparts desired characteristics.

Most of the widely used organosilanes have one organic substituent and three hydrolyzable substituents (Figure 1).

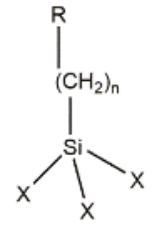

Trialkoxysilane

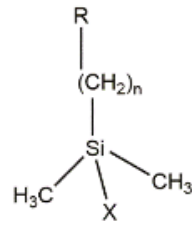

Monoalkoxysilane

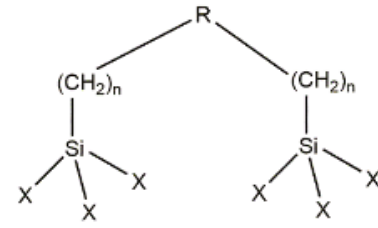

Dipodal Silane

Figure 1. Chemical formula of silane coupling agents.

Relying on the literature [17-19], the most promising strategies deal with the elaboration of silica-based structures that incorporate different functional entities, such as dyes for fluorescence imaging, magnetic complexes for magnetic resonance imaging (MRI), radioactive elements for scintigraphy or curietherapy, heavy elements for interacting with X- or g-rays, neutron absorbers for neutron therapy, or sensitizers for photodynamic therapy [21].

Another therapeutic application, probably the most used today, is controlled and targeted drug delivery. Also in this case, a suitable polymeric coating allows for combination of the particle with 
chemotherapeutic drugs that, once they reach the tumor area, can be released over time, thus allowing the administration of a lower dose and a more targeted action compared to the classic treatments. Like an airplane parachuting troops in a war zone, the polymer used to coat the actual drug plays an important role within the body.

\subsection{Biological Applications}

Molecular imprinting is one of the biological applications of the siloxanes. This specific area has developed a method for the rapid fabrication of organic polymeric and inorganic network-structured materials that act as artificial antibodies (selectively binding a template molecule). The field has progressed rapidly, and various molecular imprints selective for protein ligands have been successfully reported. For inorganic imprints, silicon-based oxides are commonly utilized (Figure 2).

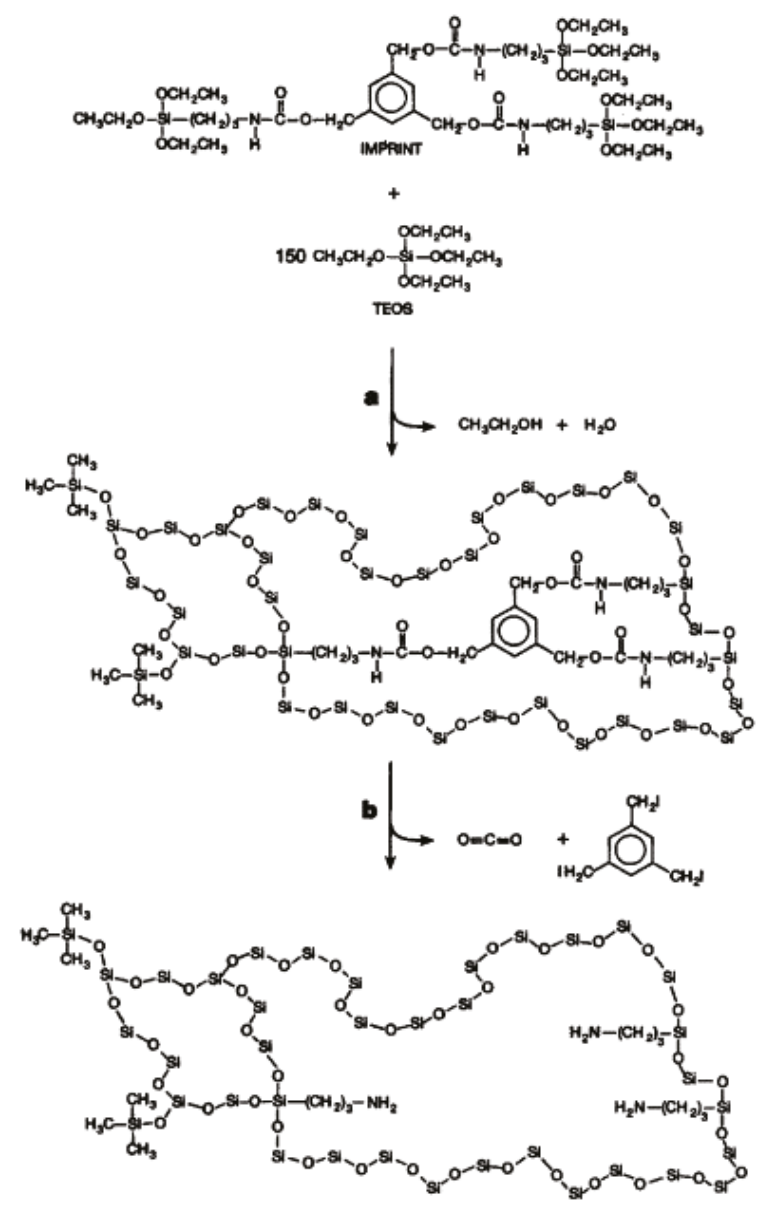

Figure 2. The formation of a covalent inorganic molecular imprint. (a) Sol-gel hydrolysis and condensation. (b) Removal of the covalently bound template. Reprinted from [22], (c) 2007 with permission from Elsevier.

Silane derivatives are allowed to hydrolyze, typically under mildly acidic conditions, in the presence of the template to form a network sol-gel structure. Usually, a mixture of tetraethoxysilane (TEOS) and functionalized organic-inorganic "hybrid" alkoxysilanes, such as 3-aminopropyltriethoxysilane 
(APTES), is employed [22]. Hydrolysis of the TEOS generates the bulk of the rigid silica framework, and the organic functionality in the hybrid silane provides the specific interactions with the template. Intense activity elaboration of fluorescent nanoparticles for replacing the organic dyes, commonly used in biological material labelling, has been devoted by the research group of Professor Tillement. To achieve efficient protection of the $\mathrm{Gd}_{2} \mathrm{O}_{3}: \mathrm{Tb}$ core and covalent linkage of biotargeting groups, encapsulation by a polysiloxane shell containing amino groups appeared to be the most appropriate strategy, due to the chemical affinity between the core and the shell, which ensures a strong interaction among both components [23]. The protective coating was prepared by sequential hydrolysis of a mixture of APTES and TEOS. The latter was chosen as polysiloxane shell precursor for introduction at the outer surface of the particle amino groups, which should act as an anchoring site for biomolecules and for reticulating the oxopolymer network. They synthesized, by a two-step route, multifunctional $\mathrm{Gd}_{2} \mathrm{O}_{3}$ nanoparticles doped by $\mathrm{Tb}^{3+}$ ions $\left(\mathrm{Gd}_{2} \mathrm{O}_{3}: \mathrm{Tb}\right)$ and protected by a silica shell. Terbium-doped gadolinium oxide nanoparticles were obtained by applying a modified "polyol" protocol [24]. Afterward, polysiloxane shell growth was induced by hydrolysis-condensation of convenient siloxane precursors in the presence of the nanoparticles [25].

\subsection{Common Formulations in Cancer Diagnosis and Therapy}

Tan and Pfister pointed out the silicon adhesives as a critical component in transdermal drug delivery (TDD) devices, drug-loaded adhesive patches which, when applied to the skin, deliver the therapeutic agent, at a controlled rate, through the skin to the systemic circulation, and to the target organs [26]. Compared to oral or injectable dosage form counterparts, TDD presents important advantages, such as the avoidance of the first-pass metabolism by the liver and digestive system, due to the improved bioavailability of the active ingredients; a constant and monitored drug delivery; a reduced comparable dose frequency; longer therapeutic action from a single application, and reversible action (patches can be removed to reverse any adverse effects that may be caused by overdosing) [26].

Particular requirements are essential in the choice of the material for functional adhesive, which are good biocompatibility with the skin, chemical compatibility with the drug, and effective delivery of the drug. The formulation used by Tan and Pfister was based on two major components dissolved together in a nonpolar hydrocarbon solvent: a high molecular weight PDMS containing residual silanol functionality $(\mathrm{SiOH})$ on the ends of the polymer chains, and a silicate resin constituted by a three-dimensional trimethylsiloxy and hydroxyl end-blocked structure (Figure 3).

\section{Silicate resin Dimethylsiloxane polymer}

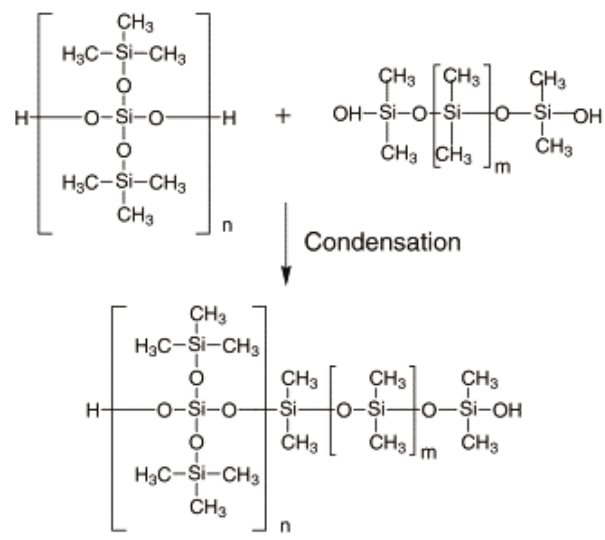

Figure 3. The chemistry of silicone pressure-sensitive adhesives (PSAs). Reprinted from [26], (c 1999 with permission from Elsevier. 
They explained their choice with the unique PDMS hybrid molecular structure and the low $T_{\mathrm{g}}$ of about $-130^{\circ} \mathrm{C}$, resulting in highly flexible and extremely open macromolecular architecture with a high void volume. This structure enables silicone adhesive to have a high permeability to vapor, gases, and a wide variety of therapeutic molecules [27].

To improve cohesive strength, Bhatt and Raul proposed the incorporation of reinforcing fillers, such as finely divided silica [28], whilst to enhance the release of drugs, they managed the degree of crosslinking in the silicone polymer matrix, thus improving cohesive strength with corresponding changes in tack, adhesion, and drug release properties.

Carelli et al. used water-soluble additives, such as ethylene glycol, glycerine, and polyethylene glycols to control the water sorption into the PDMS matrix, and to enhance the release of active agents [29].

By studying the dynamics and regulation of amino acid transportation in living organisms, Barboiu and collaborators tested whether fixed-site heteropolysiloxane membranes containing grafted macrocyclic receptors (Figure 4) can separate a mixture of amino acids having an intermediate configuration between liquid membranes (selective complexation by a specific carrier) and solid membranes (charge interactions) [30].

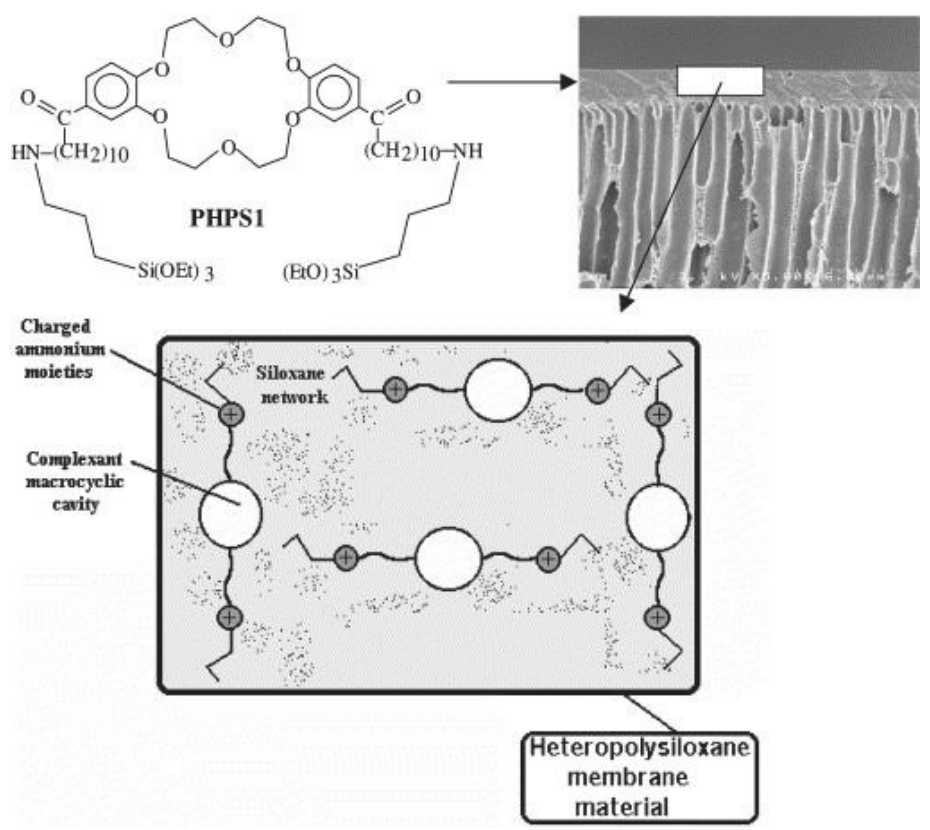

Figure 4. Scheme of fixed-site macrocyclic complexant membranes used in transport experiments. Reprinted from [30], (c) 2000 with permission from Elsevier.

They obtained high selectivities for lipophilic acidic amino acids, although they, as well as the fluxes, decreased when the $\mathrm{pH}$ of the feed phase increased. The proposed membranes presented a long lifetime, which overcomes the instability of liquid membranes, resulting in a suitable alternative to the usual chromatographic methods employed for the separation of amino acids in a cascade membrane system-type.

Underhill et al. synthesized oil-filled nanocapsules, with the aim to produce a system for drug detoxification therapy, using the oil droplets of an oil-in-water $(\mathrm{O} / \mathrm{W})$ microemulsion as templates obtaining a polysiloxane/silicate shell (Figure 5), imparting stability against coalescence, at the surface of the oil droplet by crosslinking n-octadecyltrimethoxysilane and tetramethoxysiloxane [31]. 


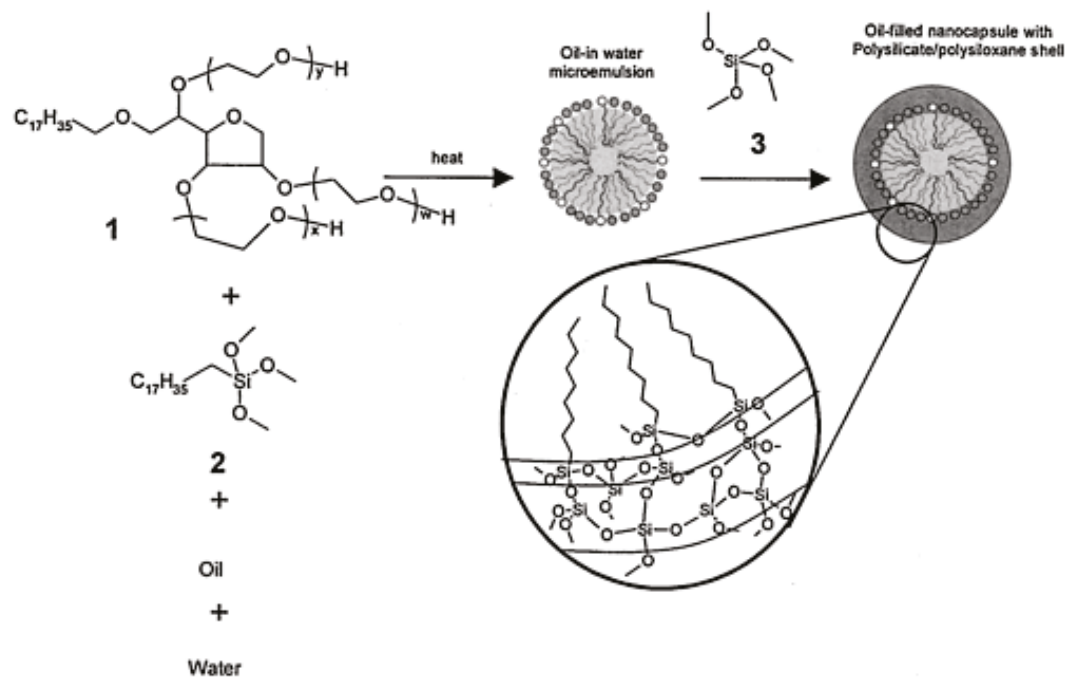

Figure 5. Formation of a mixed surfactant oil-in-water $(\mathrm{O} / \mathrm{W})$ microemulsion using Brij 97 ((1), filled circles, $\mathrm{w}+\mathrm{x}+\mathrm{y}=10)$; and $n$-octadecyltrimethoxysilane ((2), open circles), followed by the formation of a stable shell through condensation of tetramethoxysiloxane (3) around the microemulsion droplet. The inset illustrates the polysiloxane/silicate network forming the shell. Reprinted from [31], (c) 2002 with permission from ACS Publications.

They successfully tested the nanocapsules to sequester hydrophobic compounds from saline, quinoline, and free bupivacaine. The spherical shell fortifies the microemulsion droplets against coagulation or rupture, and the dispersions have a trimodal size distribution of micelles, microemulsion droplets, and nanocapsules.

Sahay et al. in their review suggested that polymers and nanomaterials can also influence intracellular transport [32], and subsequently, Nishikawa and his colleagues showed the selectively action with respect to the caveolae in human aortic endothelial cells of amphiphilic self-assembled poly(3-aminopropyl)siloxane (PAPS) of nanometric dimensions, modified with stearic acid residues and galactose [33]. They found that polysiloxane nanoparticles, endocytosed in human aortic endothelial cells, enhanced the nitric oxide release followed by the cellular uptake of the nanoparticles. The Japanese research group also confirmed that endothelial nitric oxide synthase (eNOS) was activated during cellular uptake of the nanoparticles, thus suggesting that delivery of the polymeric nanoparticles to endothelial cells can lead to the induction of nitric oxide release (Figure 6).

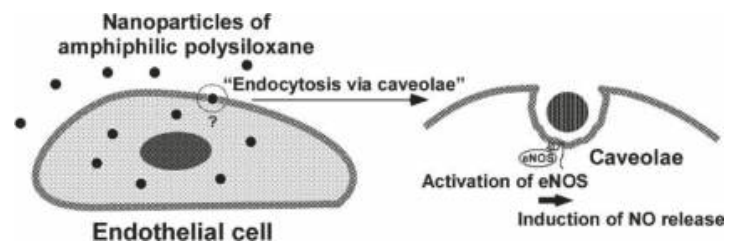

Figure 6. Scheme of nitric oxide release mechanisms by endocytosis via caveolae. Reprinted from [33], (C) 2002 with permission from ACS Publications.

In the last years, an important contribution to the theranostics development was given by the group of Lyon, coordinated by the Professor Perriat and Tillement. French researchers used 
a polysiloxane shell for encapsulate nanometric hybrid gadolinium oxide particles (Gado-6Si-NP) for diagnostic and therapeutic applications. Quantitative biodistribution using gamma-counting of each sampled organ confirmed that these nanoparticles circulated freely in the blood pool, and were rapidly cleared by renal excretion without accumulation in liver and reticuloendothelial system (RES) uptake, enabling them to be developed as multimodal agents for in vivo imaging and theranostics in oncological applications [34]. Professor Perriat and his collaborators proposed then a top-down method consisting in the fragmentation of nanometric structures consisting of core (gadolinium oxide)-shell (polysiloxane) particles with several features and functionalities, but too large in size to escape hepatic clearance [34]. The starting structures displayed an average core size of $3.5 \mathrm{~nm}$ and a shell thickness of $0.5 \mathrm{~nm}$. The fluorophore-encapsulated shell was rendered functionally active by modified 1,4,7,10-tetraazacyclododecane-1,4,7,10-tetraacetic acid (DOTA) ligands which are able to chelate core gadolinium ions (Figure 7). In aqueous solutions, the ligands strongly accelerate the core dissolution leading to a hollow polysiloxane sphere. This latter structure collapses and fragments into small and rigid platforms (SRPs) of polysiloxane that possess all the properties of the initial structure, bearing on their surface DOTA molecules that are partly chelated to dissolved gadolinium cations [19].

These SRP nanoparticles are excreted in a still significant proportion by the hepatic route (feces) and present an unexpectedly long circulation in the blood stream and completely evade uptake by the reticuloendothelial system. In vivo experiments with rats showed that the particles can accumulate in tumors by the electron paramagnetic resonance (EPR) effect, allowing for efficient radiotherapy guided by magnetic resonance imaging (MRI) [35,36], confirming that SRPs constitute an advance for more selective therapy and personalized medicine.
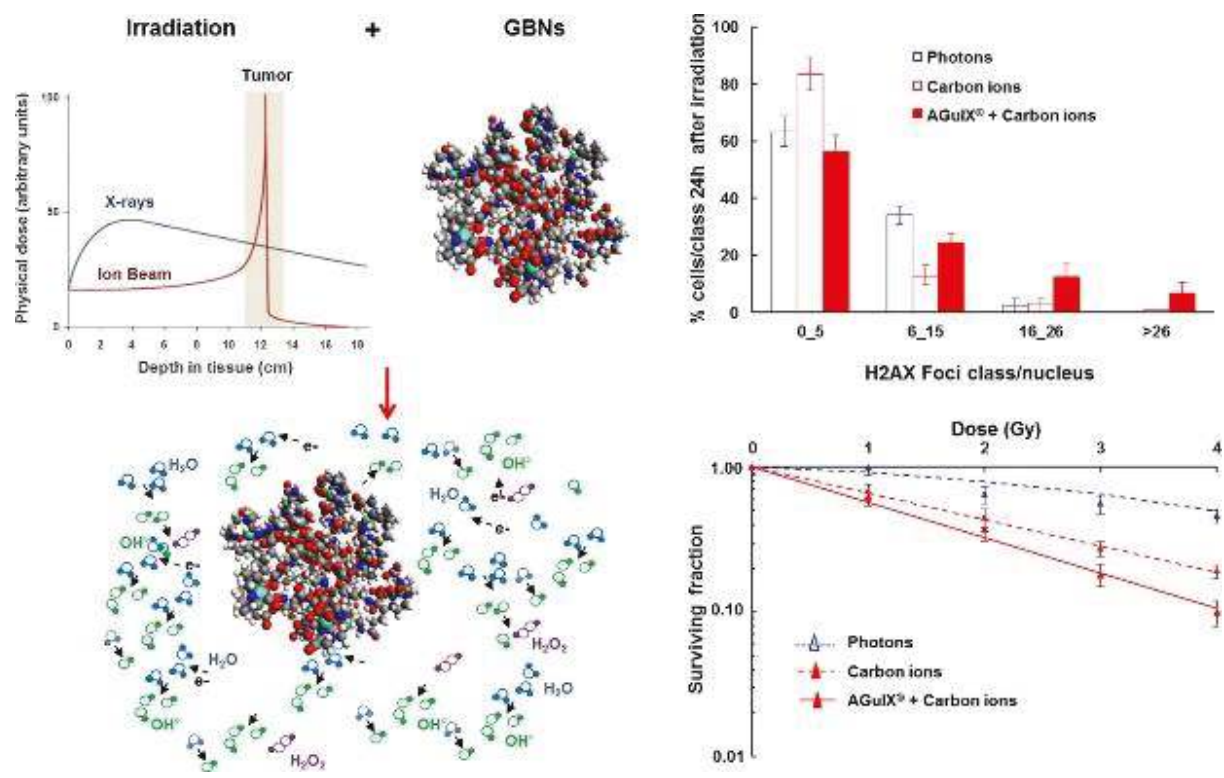

Figure 7. Representation of the AGuIX nanoparticle. Gadolinium-based nanoparticles AGuIX ${ }^{\circledR}$ combined with photon or carbon ions exposure increase irreversible DNA double-strand breaks, leading to cell death in HNSCC cells, thus representing a promising alternative in hadrontherapy. Reprinted from [37], (c) 2017 with permission from Elsevier. 


\section{Conclusions}

Theranostics has represented, for some years, the new frontier of oncology. In this short review, it has been seen how silica nanoparticles coated with siloxane polymers can be exploited both for the early detection of tumor cells and for their destruction, simultaneously performing diagnosis and therapy, as multifunctional entities. These nanoparticles allow the detection of the tumor when it has been developed only at the level of a few cells, which is impossible with simple diagnostics. It has been highlighted, both the importance of the nanoparticles' nature and the importance of an appropriate polymer coating, which allows the drug to be anchored to the particle, which can then be released gradually over time without deteriorating its therapeutic abilities. Dual use particles/device lead to a personalized medicine, thus changing the entire healthcare scene.

Author Contributions: Conceptualization, data curation, writing—original draft preparation, review and editing were performed by I.B.

Conflicts of Interest: No conflict of interest.

\section{References}

1. Voronkov, M.G.; Zelchan, G.I.; Lukevits, E.J. Silicon and Life, 2nd ed.; Zinatne Publishing: Vilnius, Lithuania, 1977.

2. Simon, T.L.; Volcani, B. Silicon and Siliceous Structures in Biological Systems; Springer: New York, NY, USA, 1981.

3. Mojsiewicz-Pieńkowska, K. Review of Current Pharmaceutical Applications of Polysiloxanes (Silicones). In Handbook of Polymers for Pharmaceutical Technologies; Kumar Thakur, V., Kumari Thakur, M., Eds.; Scrivener Publishing LLC: Beverly, MA, USA, 2015; Volume 2, pp. 363-382. [CrossRef]

4. Abbasi, F.; Mirzadeh, H.; Katbab, A.-A. Modification of polysiloxane polymers for biomedical applications: A review. Polym. Int. 2001, 50, 1279-1287. [CrossRef]

5. Blanco, I.; Bottino, F.A.; Cicala, G.; Latteri, A.; Recca, A. A kinetic study of the thermal and thermal oxidative degradations of new bridged POSS/PS nanocomposites. Polym. Degrad. Stabil. 2013, 98, 2564-2570. [CrossRef]

6. Blanco, I.; Bottino, F.A.; Abate, L. Influence of $n$-alkyl substituents on the thermal behaviour of Polyhedral Oligomeric Silsesquioxanes (POSSs) with different cage's periphery. Thermochim. Acta 2016, 623, 50-57. [CrossRef]

7. Pichaimani, P.; Krishnan, S.; Song, J.-K.; Muthukaruppan, A. Bio-silicon reinforced siloxane core polyimide green nanocomposite with multifunctional behaviour. High Perform. Polym. 2018, 30, 549-560. [CrossRef]

8. Lazzara, G.; Cavallaro, G.; Panchal, A.; Fakhrullin, R.; Stavitskaya, A.; Vinokurov, V.; Lvov, Y. An assembly of organic-inorganic composites using halloysite clay nanotubes. Curr. Opin. Colloid Interface Sci. 2018, 35, 42-50. [CrossRef]

9. Mark, J.E. Some Interesting Things about Polysiloxanes. Acc. Chem. Res. 2004, 37, 946-953. [CrossRef] [PubMed]

10. Kevadiya, B.D.; Woldstad, C.; Ottemann, B.M.; Dash, P.; Sajja, B.R.; Lamberty, B.; Morsey, B.; Kocher, T.; Dutta, R.; Bade, A.N.; et al. Multimodal Theranostic Nanoformulations Permit Magnetic Resonance Bioimaging of Antiretroviral Drug Particle Tissue-Cell Biodistribution. Theranostics 2018, 8, 256-276. [CrossRef] [PubMed]

11. Stafford, S.; Serrano Garcia, R.; Gun'ko, Y.K. Multimodal Magnetic-Plasmonic Nanoparticles for Biomedical Applications. Appl. Sci. 2018, 8, 97. [CrossRef]

12. Xu, Z.; Ma, X.; Gao, Y.-E.; Hou, M.; Xue, P.; Li, C.M.; Kang, Y. Multifunctional silica nanoparticles as a promising theranostic platform for biomedical applications. Mater. Chem. Front. 2017, 1, 1257-1272. [CrossRef]

13. Storm, F.K.; Morton, D.L. Localized Hyperthermia in the Treatment of Cancer. CA Cancer J. Clin. 1983, 33, 44-56. [CrossRef] [PubMed]

14. Riviere, C.; Roux, S.; Tillement, O.; Billotey, C.; Perriat, P. Nanosystems for medical applications: Biological detection, drug delivery, diagnosis and therapy. Ann. Chim. Sci. Mater. 2006, 31, 351-367. [CrossRef] 
15. Godin, B.; Sakamoto, J.H.; Serda, R.E.; Grattoni, A.; Bouamrani, A.; Ferrari, M. Emerging applications of nanomedicine for the diagnosis and treatment of cardiovascular diseases. Trends Pharmacol. Sci. 2010, 31, 199-205. [CrossRef] [PubMed]

16. Mrówczynski, R.; Turcu, R.; Leostean, C.; Scheidt, H.A.; Liebscher, J. New versatile polydopamine coated functionalized magnetic nanoparticles. Mat. Chem. Phys. 2013, 138, 295-302. [CrossRef]

17. Xie, J.; Chen, K.; Huang, J.; Lee, S.; Whang, J.; Gao, J.; Li, X.; Chen, X. PET/NIRF/MRI triple functional iron oxide nanoparticles. Biomaterials 2010, 31, 3016-3022. [CrossRef] [PubMed]

18. Rieter, W.J.; Kim, J.S.; Taylor, K.M.L.; An, H.; Lin, W.; Tarrant, T.; Lin, W. Hybrid silica nanoparticles for multimodal imaging. Angew. Chem. Int. Ed. 2007, 46, 3680-3682. [CrossRef] [PubMed]

19. Bridot, J.L.; Faure, A.C.; Laurent, S.; Riviere, C.; Billotey, B.; Hiba, C.; Janier, M.; Josserand, V.; Coll, J.L.; Vander Elst, L.; et al. Hybrid gadolinium oxide nanoparticles: Multimodal contrast agents for in vivo imaging. J. Am. Chem. Soc. 2007, 129, 5076-5084. [CrossRef] [PubMed]

20. Jenaa, K.K.; Narayanb, R.; Alhassana, S.M. Highly branched graphene siloxane-polyurethane-urea (PU-urea) hybrid coatings. Prog. Org. Coat. 2017, 111, 343-353. [CrossRef]

21. Lux, F.; Mignot, A.; Mowat, P.; Louis, C.; Dufort, S.; Bernhard, C.; Denat, F.; Boschetti, F.; Brunet, C.; Antoine, R.; et al. Ultrasmall Rigid Particles as Multimodal Probes for Medical Applications. Angew. Chem. Int. Ed. 2011, 50, 12299-12303. [CrossRef] [PubMed]

22. Hansen, D.E. Recent developments in the molecular imprinting of proteins. Biomaterials 2007, 28, 4178-4191. [CrossRef] [PubMed]

23. Sanchez, C.; Soler-Illia, G.J.A.A.; Ribot, F.; Lalot, T.; Mayer, C.R.; Cabuil, V. Designed Hybrid Organic-Inorganic Nanocomposites from Functional Nanobuilding Blocks. Chem. Mater. 2001, 13, 3061-3083. [CrossRef]

24. Bazzi, R.; Flores, M.A.; Louis, C.; Lebbou, K.; Zhang, W.; Dujardin, C.; Roux, S.; Mercier, B.; Ledoux, G.; Bernstein, E.; et al. Synthesis and properties of europium-based phosphors on the nanometer scale: $\mathrm{Eu}_{2} \mathrm{O}_{3}$, $\mathrm{Gd}_{2} \mathrm{O}_{3}: \mathrm{Eu}$, and $\mathrm{Y}_{2} \mathrm{O}_{3}:$ Eu. Colloid Interface Sci. 2004, 273, 191-197. [CrossRef] [PubMed]

25. Louis, C.; Bazzi, R.; Marquette, C.A.; Bridot, J.-L.; Roux, S.; Ledoux, G.; Mercier, B.; Blum, L.; Perriat, P.; Tillement, O. Nanosized Hybrid Particles with Double Luminescence for Biological Labeling. Chem. Mater. 2005, 17, 1673-1682. [CrossRef]

26. Tan, H.S.; Pfister, W.R. Pressure-sensitive adhesives for transdermal drug delivery systems. Pharm. Sci. Technol. Today 1999, 2, 60-69. [CrossRef]

27. Ulman, K.L.; Thomas, X. Advances in Pressure Sensitive Adhesive Technology—2; Satas, D., Ed.; Stanford Libraries: Warwick, NY, USA, 1995; pp. 133-157. ISBN 978-0963799326.

28. Bhatt, P.P.; Raul, V.A. Method of Controlling Release of an Active or Drug from a Silicone Rubber Matrix. U.S. Patent 5,597,584, 28 January 1997.

29. Carelli, V.; Di Colo, G. Effect of Different Water-Soluble Additives on Water Sorption into Silicone Rubber. J. Pharm. Sci. 1983, 73, 316-317. [CrossRef]

30. Barboiu, M.; Guizard, C.; Hovnanian, N.; Palmeri, J.; Reibel, C.; Cot, I.; Luca, C. Facilitated transport of organics of biological interest I. A new alternative for the separation of amino acids by fixed-site crown-ether polysiloxane membranes. J. Membr. Sci. 2000, 172, 91-103. [CrossRef]

31. Underhill, R.S.; Jovanovic, A.V.; Carino, S.R.; Varshney, M.; Shah, D.O.; Dennis, D.M.; Morey, T.E.; Duran, R.S. Oil-Filled Silica Nanocapsules for Lipophilic Drug Uptake: Implications for Drug Detoxification Therapy. Chem. Mater. 2002, 14, 4919-4925. [CrossRef]

32. Sahay, G.; Alakhova, D.Y.; Kabanov, A.V. Endocytosis of nanomedicines. J. Controlled Release 2010, 145, 182-195. [CrossRef] [PubMed]

33. Nishikawa, T.; Iwakiri, N.; Kaneko, Y.; Taguchi, A.; Fukushima, K.; Mori, H.; Morone, N.; Kadokawa, J.-I. Nitric oxide release in human aortic endothelial cells mediated by delivery of amphiphilic polysiloxane nanoparticles to caveolae. Biomacromolecules 2009, 10, 2074-2085. [CrossRef] [PubMed]

34. Kryza, D.; Taleb, J.; Janier, M.; Marmuse, L.; Miladi, I.; Bonazza, P.; Louis, C.; Perriat, P.; Roux, S.; Tillement, O.; et al. Biodistribution Study of Nanometric Hybrid Gadolinium Oxide Particles as a Multimodal SPECT/MR/Optical Imaging and Theragnostic Agent. Bioconjugate Chem. 2011, 22, 1145-1152. [CrossRef] [PubMed] 
35. Mignot, A.; Truillet, C.; Lux, F.; Sancey, L.; Louis, C.; Denat, F.; Boschetti, F.; Bocher, L.; Gloter, A.; Stéphan, O.; et al. A top-down synthesis route to ultrasmall multifunctional Gd-based silica nanoparticles for theranostic applications. Chem. Eur. J. 2013, 19, 6122-6136. [CrossRef] [PubMed]

36. Sancey, L.; Lux, F.; Kotb, S.; Roux, S.; Dufort, S.; Bianchi, A.; Crémillieux, Y.; Fries, P.; Coll, J.-L.; Rodriguez-Lafrasse, C.; et al. The use of theranostic gadolinium-based nanoprobes to improve radiotherapy efficacy. Br. J. Radiol. 2014, 87, 20140134. [CrossRef] [PubMed]

37. Wozny, A.S.; Aloy, M.T.; Alphonse, G.; Magné, N.; Janier, M.; Tillement, O.; Lux, F.; Beuve, M.; Rodriguez-Lafrasse, C. Gadolinium-based nanoparticles as sensitizing agents to carbon ions in head and neck tumor cells. Nanomed. Nanotechnol. Biol. Med. 2017, 13, 2655-2660. [CrossRef] [PubMed]

(C) 2018 by the author. Licensee MDPI, Basel, Switzerland. This article is an open access article distributed under the terms and conditions of the Creative Commons Attribution (CC BY) license (http://creativecommons.org/licenses/by/4.0/). 
Article

\title{
Structural and Thermoanalytical Characterization of 3D Porous PDMS Foam Materials: The Effect of Impurities Derived from a Sugar Templating Process
}

\author{
José González-Rivera ${ }^{1}$, Rossella Iglio ${ }^{1}$, Giuseppe Barillaro ${ }^{1, *}$, Celia Duce ${ }^{2}$ and \\ Maria Rosaria Tinè ${ }^{2}$ \\ 1 Department of Information Engineering, University of Pisa, via G. Caruso 16, 56122 Pisa, Italy; \\ jose.gonzalezrivera@for.unipi.it (J.G.-R.); rossella.iglio@ing.unipi.it (R.I.) \\ 2 Department of Chemistry and Industrial Chemistry, University of Pisa, Via Moruzzi 3, 56124 Pisa, Italy; \\ mariarosaria.tine@unipi.it \\ * Correspondence: g.barillaro@iet.unipi.it (G.B.); celia.duce@unipi.it (C.D.); \\ Tel.: +39-050-2217-601 (G.B.); +39-050-2219-311 (C.D.)
}

Received: 15 May 2018; Accepted: 1 June 2018; Published: 5 June 2018

\begin{abstract}
Polydimethylsiloxane (PDMS) polymers are extensively used in a wide range of research and industrial fields, due to their highly versatile chemical, physical, and biological properties. Besides the different two-dimensional PDMS formulations available, three-dimensional PDMS foams have attracted increased attention. However, as-prepared PDMS foams contain residual unreacted low molecular weight species that need to be removed in order to obtain a standard and chemically stable material for use as a scaffold for different decorating agents. We propose a cleaning procedure for PDMS foams obtained using a sugar templating process, based on the use of two different solvents (hexane and ethanol) as cleaning agents. Thermogravimetry coupled with Fourier Transform Infrared Spectroscopy (TG-FTIR) for the analysis of the evolved gasses was used to characterize the thermal stability and decomposition pathway of the PDMS foams, before and after the cleaning procedure. The results were compared with those obtained on non-porous PDMS bulk as a reference. Micro-CT microtomography and scanning electron microscopy (SEM) analyses were employed to study the morphology of the PDMS foam. The thermogravimetric analysis (TGA) revealed a different thermal behaviour and crosslinking pathway between bulk PDMS and porous PDMS foam, which was also influenced by the washing process. This information was not apparent from spectroscopic or morphological studies and it would be very useful for planning the use of such complex and very reactive systems.
\end{abstract}

Keywords: PDMS; sugar templating process; 3D porous network; thermal stability; TG-FTIR; X-ray (Micro-CT) microtomography

\section{Introduction}

Polydimethylsiloxanes are organosilicon polymers commonly used in a wide range of industrial, biomedical, and medicinal or pharmaceutical applications, either in pure form or as formulations. Their structural features ( $\mathrm{Si}-\mathrm{O}-\mathrm{Si}$ angles; $\mathrm{Si}-\mathrm{O}$ bond length, dissociation energy, and freedom of rotation; weak intermolecular forces) make them very flexible polymers with unique physical and chemical characteristics $[1,2]$. They exhibit low glass transition temperatures $\left(T_{\mathrm{g}}\right)$, good resistance to thermal and oxidative degradation, good permeability to gas, and good dielectric properties. Their low surface tension makes them excellent surface active agents. They are also biocompatible, with low toxicity, and therefore suitable for many physiological and biomedical purposes [2].

Different types of PDMSs are used for various applications, such as silicone oils, bulk PDMS, and porous PDMS. 
Commercially available silicone oils are typically used as protective coatings for industrial substrates, thanks to their chemical and physical properties as well as their ability to form thin films. They can be easily modified to form hybrid, nano-composite materials suitable for use as anticorrosives [3], ice-retarding [4], self-cleaning/antireflective [5], and, flame/heat/fire retardants [6], or in enzyme immobilization to obtain biocatalytic paints with antifouling/antibiofouling properties [7].

Bulk PDMS is widely used in biomedical applications, thanks to its good blood compatibility, low toxicity, and good thermal and oxidative stability. Medical devices made in PDMS include mammary prostheses [8], cell bioreactors [9], contact lenses [10], and microfluidic devices [11]. In addition, the relatively low Young's modulus of bulk PDMS (0.4 MPa) has led to the successful use of this soft polymer in the replica molding technique, for the fabrication of patterns with features on a micro and nano-scale $[12,13]$.

More recently, three-dimensional (3D) PDMS foams, namely, porous PDMS, have attracted attention in areas such as medicine, chemistry, materials science, and engineering. Due to its unique properties and easy fabrication, porous PDMS has been used in many applications, such as oil/water separation [14-17], cellular scaffolds [18], microfluidic pumps [19-21], and stress strain sensors [22,23].

3D PDMS foams with either ordered or random porous skeletons have been reported. For instance, Duan et al. used a 3D printing technique to prepare regular, porous polylactic acid scaffolds which were used as a template to create 3D ordered porous PDMS foams. The 3D PDMS was then integrated with a carbon nanotube/graphene network to obtain a stretchable strain sensor [23]. 3D PDMS foams with a random porous skeleton are easily fabricated by replicating the structure of a different kind of random, porous sacrificial template. For instance, Chen et al., used a nickel foam as a 3D template. They replicated its architecture by impregnation method using diluted PDMS (as a solution), achieving a highly porous framework with continuous macropores [24].

Several materials, such as common sugar cubes or grains, solid particles of citric acid monohydrate (CAM), and salts (like $\mathrm{NaCl}$ ) can be used for the easy, low cost, and eco-friendly preparation of porous PDMS $[14,15,17,18,21,22]$. These preparation methods should meet two chief criteria: (1) solvent wettability to PDMS and, (2) template solubility in the solvent [17]. The most commonly used solvents are ethanol and water.

A blend of PDMS prepolymer and sacrificial material is usually prepared, which is casted and polymerized on a suitable template, and the sacrificial material is then dissolved. The time required to remove the sacrificial materials can be quite long, especially when its concentration is not high enough to ensure the formation of a connected domain within the polymer. For instance, Yu et al., prepared a porous PDMS sponge for oil/water separation by directly mixing CAM particles with PDMS prepolymer. After polymerization, the samples were immersed in ethanol for $6 \mathrm{~h}$ to remove the CAM particles [17]. The authors thus obtained a PDMS sponge with an excellent 3D interconnected porous structure and high oil/water separation efficiency. Li et al., obtained PDMS-based 3-D scaffolds containing interconnected micro- and macro-pores for tissue engineering applications, by blending a dispersion of ethanol and $\mathrm{NaCl}$ particles with a PDMS prepolymer. To remove the $\mathrm{NaCl}$ particles, the samples were immersed for three days in water [18]. Zhang et al., prepared a PDMS oil absorbent using a sugar template method, directly mixing the sugar particles with PDMS prepolymer and $\mathrm{p}$-Xylene. After polymerization, the sugar particles were dissolved with warm water and the p-Xylene was removed with ethanol [16].

The use of pre-formed sacrificial templates, such as sugar cubes, facilitates the infiltration of pure PDMS prepolymer completely within the template and, in turn, a faster template dissolution after polymerization, for example using warm deionized water $[15,16,21,22]$. In fact, the pre-formed sacrificial template ensures a connected path for the sacrificial material, which speeds up sugar dissolution.

The aim of this work was to develop a cleaning procedure for 3D porous PDMS foam obtained using a sugar templating process in order to achieve a standard and chemically stable material 
to be used as a scaffold for different decorating agents. In fact, according to our experimental experience, the treatment of PDMS foam with hot water is often not enough to obtain a chemically stable PDMS. Residues of sugar, unreacted base and curing agents, low molecular weight oligomers may be introduced in the foam which then lead to a continuous mass loss of the foam during the deposition of various decorating agents by drop-casting from hexane or ethanol.

We propose two solvents as cleaning agents: hexane and ethanol. The cleaning level of the foam was assessed by analyzing the composition of the waste washing solvents by Attenuated total reflectance-Fourier transform infrared (ATR-FTIR) spectroscopy. The morphology of the PDMS foam was characterized by SEM analysis and X-ray micro-CT microtomography. The thermal stability and thermal decomposition pathway of the foam were assessed by thermogravimetry.

The study of thermal decomposition behaviour has been mainly carried out in the literature for bulk PDMS with a different level and type of cross-linker agents [25], different molecular weights [26], or catalyst free PDMS [27].

The thermal degradation of PDMS has been mostly investigated under inert atmosphere, which results in depolymerization, through the $\mathrm{Si}-\mathrm{O}$ bond scission, leading to the formation of a mixture of different cyclic oligomers as degradation products. In air, $\mathrm{CO}_{2}$ and water are also present [28]. Camino et al., showed that the products of thermal degradation of PDMS are influenced by the temperature and heating rate [29]. At high temperatures, a radical mechanism occurs, through homolytic $\mathrm{Si}-\mathrm{CH}_{3}$ scission. The formation of macro-radicals leads to cross-linking with the formation of ceramic silicon-oxicarbide.

On the other hand, the thermal stability of PDMS is also influenced by the presence of impurities (even at a trace level). If traces of oxygen, moisture, or terminal hydroxyl groups are present in the PDMS scaffold, thermal depolymerization under inert atmosphere occurs at different lower temperatures and produces various decomposition products [27].

There is therefore a need to assess the thermal degradation behaviour and products of decomposition of 3D porous PDMS under different conditions.

In our work, we used thermogravimetry coupled with FTIR (TG-FTIR) for the analysis of the evolved gasses in order to characterize the thermal stability and decomposition pathway of porous PDMS foam before and after the washing procedure. The same cleaning procedure was also applied to PDMS bulk samples as a reference, and the results on a porous and non-porous material were compared.

\section{Materials and Methods}

\subsection{Materials}

Common sugar cubes (Dietor vantaggio), PDMS, Sylgard 184 base and thermal curing agent (containing a Pt-based catalyst) were purchased from Dow Corning Corporation (Wiesbaden, Germany). Hexane (>95\%) and ethanol (99.8\%) were purchased from Sigma-Aldrich (Milan, Italy) and used as received.

\subsection{Preparation of Porous PDMS Foam}

The 3D porous PDMS framework was prepared according to the methodology reported in $[22,30]$. Briefly: a sugar cube was placed in a Petri dish containing a mixture of PDMS prepolymer using a 10:1 ratio of base: curing components by weight. The Petri dish was then put in a vacuum chamber for $2 \mathrm{~h}$ to allow for complete PDMS capillary infiltration into the sugar template and to remove trapped air bubbles. Sugar cubes infiltrated with the PDMS prepolymer mixture were placed in a convection oven for $4 \mathrm{~h}$ at $65^{\circ} \mathrm{C}$ to ensure full PDMS polymerization through thermal curing. After the in situ polymerization, the PDMS-sugar templating scaffold composite was then placed in a freezer for $3 \mathrm{~min}$ to enable PDMS detachment from the scaffold. Sugar scaffold templates were dissolved by rinsing in 
deionized water at $60^{\circ}$ for $1 \mathrm{~h}$. The resulting 3D porous PDMS foam was dried under a chemical hood for $1 \mathrm{~h}$ at room temperature.

The purification steps on both bulk PDMS and 3D porous PDMS were performed as follows: the samples were soaked and stirred in the solvent (ethanol or hexane) for $72 \mathrm{~h}$ and the free solvent removed by filtration. The swollen polymers were weighed and the swelling capacity determined according to: $\%$ wt. $=100 \times\left[\right.$ (weight of PDMS swollen - weight of $\left.\mathrm{PDMS}_{\text {dried }}\right) /$ weight of $\left.\mathrm{PDMS}_{\text {swollen }}\right]$. The polymers were then dried at $70{ }^{\circ} \mathrm{C}$ for at least $4 \mathrm{~h}$. A second cleaning step was then performed, soaking and stirring the polymeric materials with fresh solvent for $12 \mathrm{~h}$. The swelling capacity was recorded at each step of the soaking/swelling/drying purification process and a mean value was reported. Both the waste solvents and the dried PDMS-based polymers, were analysed by ATR-FTIR spectroscopy (Agilent Technologies, Milan, Italy).

\subsection{Morphological Characterization}

The cross-sections of PDMS foam were investigated using a scanning electron microscope (SEM, JEOL JSM-6390, Milan, Italy) at an accelerating voltage of $5 \mathrm{kV} . \mu-\mathrm{CT}$ three-dimensional reconstruction was performed using a SkyScan 1174 system (Skyscan, Aartselaar, Kontich, Belgium) with a resolution of $6.5 \mu \mathrm{m} \cdot$ pixel $^{-1}, 180^{\circ}$ rotation.

\subsection{Thermogravimetry}

A TA Instruments Thermobalance model Q5000IR equipped with an FTIR (Agilent Technologies, Milan, Italy) spectrophotometer Cary 640 model for evolved gas analysis (EGA) was used. TG-FTIR measurements were performed at a rate of $20{ }^{\circ} \mathrm{C} / \mathrm{min}$, from 30 to $900{ }^{\circ} \mathrm{C}$ under nitrogen flow (70 mL/min) using Pt crucibles, from 600 to $4000 \mathrm{~cm}^{-1}$ with a $4 \mathrm{~cm}^{-1}$ width slit. A background spectrum was taken before each analysis in order to zero the signal in the gas cell and to eliminate the contribution due to the amount of ambient water and carbon dioxide. Mass calibration was performed using certified mass standards, supplied by TA Instruments, in the range from 0 to $100 \mathrm{mg}$. The amount of sample in each experiment varied between 10 and $12 \mathrm{mg}$. Temperature calibration was based on the Curie point of paramagnetic metals. A multipoint calibration with five Curie points from reference materials (Alumel, Ni, Ni83\%Co17\%, Ni63\%Co37\%, Ni37\%Co63\%) was performed.

\subsection{ATR-FTIR Analysis}

Infrared spectra were recorded using an FT-IR Agilent Technologies Spectrophotometer model Cary 640 (Agilent Technologies, Milan, Italy), equipped with a universal attenuated total reflectance accessory (ATRU). A few micrograms of sample powder were used, and for the liquid samples, $20 \mu \mathrm{L}$ were dripped onto the ATR accessory with the following spectrometer parameters; resolution: $4 \mathrm{~cm}^{-1}$, spectral range: $600-4000 \mathrm{~cm}^{-1}$, number of scans: 128 . Agilent spectrum software (Agilent Technologies, Milan, Italy) was used to process the FTIR spectra. The FTIR spectra of the impurities obtained in the washing solvents were recorded after solvent evaporation.

\section{Results and Discussion}

\subsection{PDMS Crosslinking Polycondensation Reaction}

The Pt-catalysed crosslinking polycondensation reaction occurring between silane terminations $(\mathrm{Si}-\mathrm{H})$, present in the PDMS curing and, vinyl groups in the PDMS base precursor (Scheme 1) was used to prepare two different PDMS-based materials: (i) a highly porous PDMS foam with a 3D interconnected macropore framework promoted by the sugar templating process and, (ii) a non-porous crosslinked bulk PDMS.

The FTIR spectra of the curing agent, PDMS base precursor, and the two PDMS polymers (i.e., porous and non porous) materials prepared in this work are shown in Figure S1. The fingerprint of silicone (-Si-O-Si- and $-\mathrm{Si}-\mathrm{CH}_{3}$ signals, see Table 1 and Figure $\mathrm{S} 1$ ) are evident in the FTIR spectra 
of bulk PDMS and 3D porous PDMS foam. The main signals corresponding to $\mathrm{Si}-\mathrm{H}\left(2158 \mathrm{~cm}^{-1}\right.$ stretching, see Table 1 and Figure S1) present in the PDMS curing agent were not detected for either of the PDMS polymer materials, thus confirming that the crosslinking polymerization reaction was successfully carried out.

However, a small signal at $910 \mathrm{~cm}^{-1}$ was detected (see Figure S1) for both bulk PDMS and 3D porous PDMS foam. This peak can be ascribed to a small amount of residual non-cross-linked curing agent entrapped in the framework. In fact, the as-prepared materials can contain residual unreacted low molecular weight species [31] that need to be removed to obtain a standard and stable, either porous or non-porous PDMS, which can be used as a scaffold of different decorating agents.

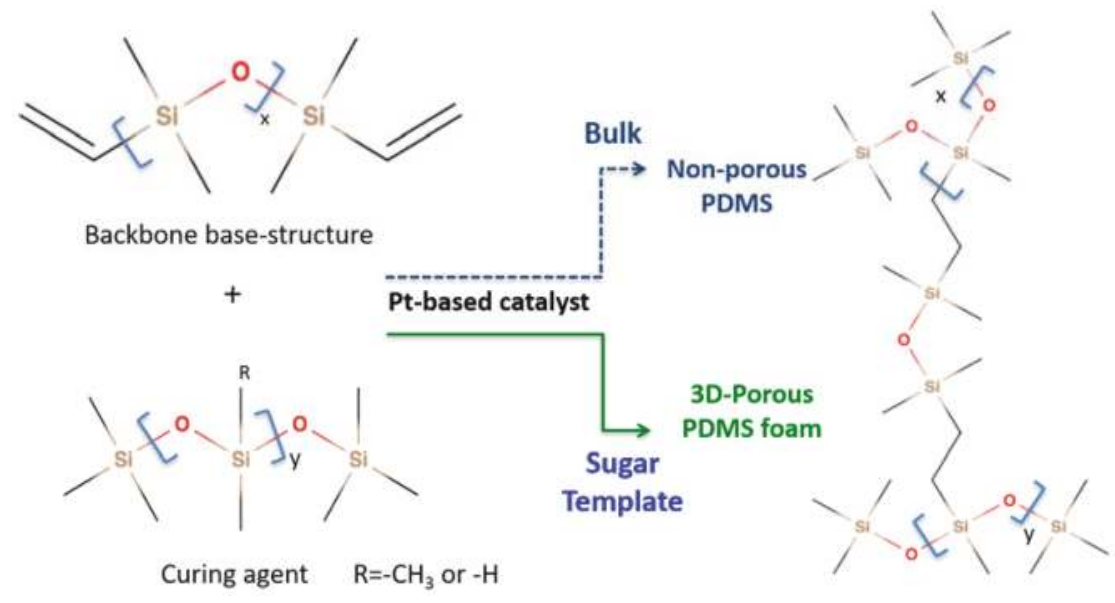

Scheme 1. The PDMS crosslinking polymerization reaction.

\subsection{Surface and Morphological Characterization of 3D Porous PDMS Foam}

The porous PDMS foam obtained by the sugar templating approach has very complex interconnected pore channels with a widely polydisperse volume along the different dimensions and an optical picture of the as prepared materials is shown in Figure 1a. The PDMS was infiltrated around the sugar grains and after the sugar templating removal, the SEM analysis highlighted that the $3 \mathrm{D}$ porous structure had a smooth internal surface area with a macro-pore size in the range of $500 \pm 300 \mu \mathrm{m}$ (Figure 1b).

The porosity of this material has recently been reported (about 77\%) and the pore sizes were in agreement with the size of the SEM analysis and those of the sugar grains used as the template [22]. Useful morphological information about different polymeric materials has reported by SEM imaging [32,33].The SEM analysis is however limited to two-dimensional views of a material, which provide insights into the surface textural properties and morphology.

The analysis of the pore size and pore size distribution of materials with a 3D interconnected channel framework, such as the 3D porous PDMS foam fabricated here, is complex and it is thus more appropriate to refer to a pore volume density. The 3D reconstruction of porous PDMS foam performed by the micro CT $x$ ray scanning is shown in Figure 1c. The analysis of cross-sectional slides (see Figure $1 \mathrm{~d}-\mathrm{f}$ ), here shown as an example of the interconnected pores at different heights: $\mathrm{z}_{\mathrm{b}}=0.86 \mathrm{~mm}, \mathrm{z}_{\mathrm{c}}=4.12 \mathrm{~mm}$ and $\mathrm{z}_{\mathrm{d}}=8.60 \mathrm{~mm}$ ) and the video (showing the whole volume and pore volume evolution along the 3D directions of the porous PDMS foam) obtained by the reconstruction of 475 cross-sectional slides along the Z dimension (see VS1) highlighted that the pore framework in the $3 \mathrm{D}$ porous PDMS foam, is an interconnected three-dimensional channel system with a pore volume density gradient that is widely distributed along the various directions. Micro CT X ray scanning is 
thus a very useful tool for morphological studies of these complex matrixes, as it gives clear visual information on their entire internal structure.

(a)

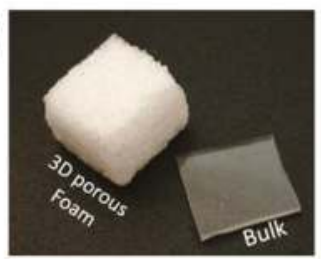

(d)

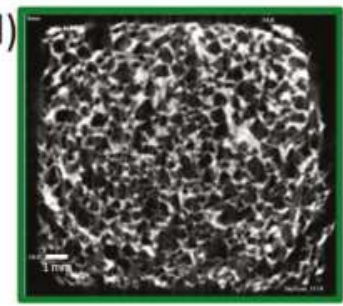

(b)
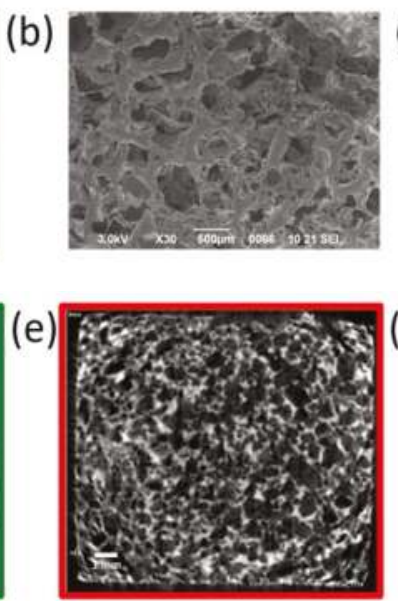

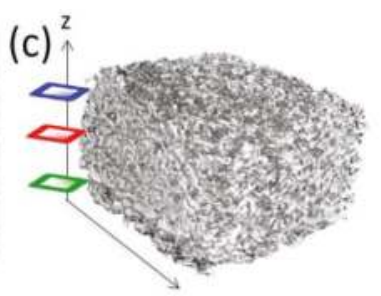

(f)

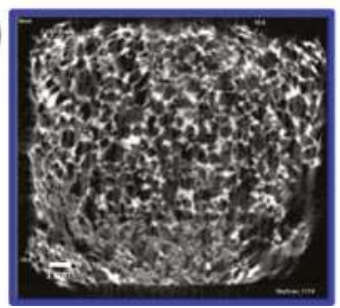

Figure 1. Morphological characterization of the 3D porous PDMS foam obtained by the sugar templating process: (a) Optical picture, (b) SEM image and, (c) 3D volume reconstruction and (d-f) cross-sectional profiles at different heights $\left(\mathrm{z}_{\mathrm{b}}=0.86 \mathrm{~mm}, \mathrm{z}_{\mathrm{c}}=4.12 \mathrm{~mm}\right.$ and $\left.\mathrm{z}_{\mathrm{d}}=8.60 \mathrm{~mm}, \mathrm{z}_{\mathrm{max}}=9.0 \mathrm{~mm}\right)$ obtained by micro CT analysis.

\subsection{The ATR-FTIR Spectra of the Waste Washing Solvents}

Hexane and ethanol were selected as cleaning agents to remove unreacted species. The cleaning level of the materials was assessed by monitoring the presence of waste compounds in the washing solvents by FTIR.

Figure 2a shows the picture of a 3D-porous PDMS foam as-prepared and after it had been swollen in ethanol and hexane. The swelling ratio (or solvent absorption capacity) is as high as $600 \mathrm{wt} \%$ in hexane and $300 \mathrm{wt} \%$ in ethanol (compared to the weight of the dried 3D-porous PDMS foam). This is significantly higher than that of the bulk PDMS prepared in this work, which was up to $90 \mathrm{wt} \%$ in hexane and $11 \mathrm{wt} \%$ in ethanol. The improved swelling capacity of 3D-porous PDMS foams with respect to bulk PDMS, can be attributed to the porosity of the material and is in agreement with data for similar PDMS-based materials [17].

The ATR-FTIR spectra of the washing solvents showed that small oligomers from the PDMS base precursor $\left(-\mathrm{Si}-\mathrm{CH}_{3},-\mathrm{Si}-\mathrm{O}-\mathrm{Si}-\right.$, see Table 1 for FTIR assignments) and curing agent $\left(-\mathrm{Si}-\mathrm{CH}_{3},-\mathrm{Si}-\mathrm{H}\right.$, -Si-O-Si-, see Table 1 for FTIR assignments) were completely removed from both porous and bulk PDMS after $72 \mathrm{~h}$ of soaking either in hexane or in ethanol (first purification step, see Figure $2 \mathrm{~b}, \mathrm{c}$ ). Mass losses of $4.3 \% \pm 0.1 \%$ (hexane) and $3.7 \% \pm 0.3 \%$ (ethanol) for bulk PDMS and of $3.8 \% \pm 0.3 \%$ (hexane) and $3.4 \% \pm 0.8 \%$ (ethanol) for 3D-porous PDMS foam were recorded after the first purification step, with no significant differences for the two solvents.

No significant impurity signals were observed in the FTIR spectra of either the porous or bulk PDMS after a further $12 \mathrm{~h}$ soaking in hexane and ethanol (see Figure $2 \mathrm{~b}, \mathrm{c}$ ). In this case, the PDMS mass losses were less than $0.1 \%$ for both the solvents.

Interestingly, ATR-FTIR spectra of both bulk PDMS and 3D porous PDMS foam acquired before and after soaking and washing in both solvents were unchanged, suggesting that the cleaning procedure does not impact on the PDMS chemical structure (see Figure S2). The FTIR spectra were characterized by $-\mathrm{Si}-\mathrm{O}-\mathrm{Si}$ and $-\mathrm{Si}-\mathrm{CH}_{3}$ typical absorptions, although $\mathrm{Si}-\mathrm{H}$ absorption was also visible, suggesting the presence of silane terminal groups in the polymer. 
(a)

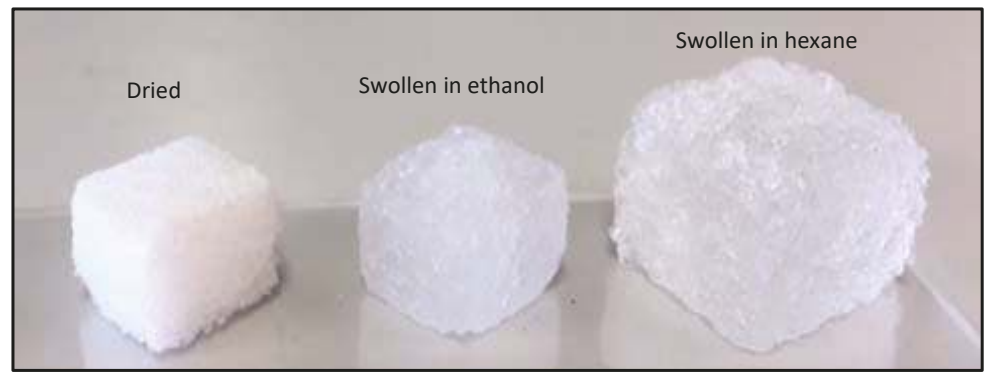

(b)
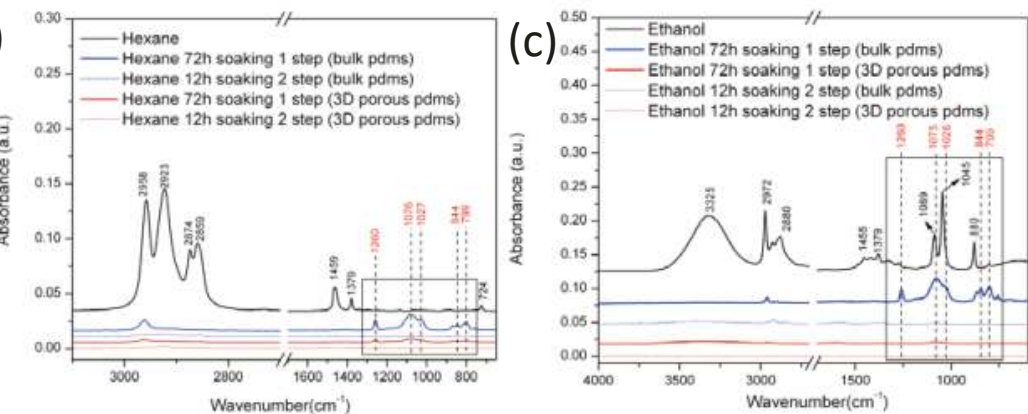

Figure 2. (a) A 3D-porous PDMS foam obtained through a sugar-based template approach and its swelling behaviour in ethanol and hexane. ATR-FTIR spectra of hexane (b) and ethanol (c) cleaning solvents, acquired during the cleaning procedure setup for both bulk PDMS and 3D-porous PDMS foam.

Table 1. Assignments of the main FTIR absorption bands of silicon-based materials.

\begin{tabular}{|c|c|c|}
\hline Wavenumber $\left(\mathrm{cm}^{-1}\right)$ & Assignment & Reference \\
\hline \multicolumn{3}{|c|}{$\mathrm{Si}-\mathrm{CH}_{3}, \mathrm{Si}-\mathrm{CH}_{2}-$} \\
\hline 690,790 & Si-C stretching & [34] \\
\hline 843 & $\mathrm{C}-\mathrm{H}\left(-\mathrm{CH}_{2}\right)$ rocking & [25] \\
\hline 1260 & symmetric $\mathrm{C}-\mathrm{H}$ bending & [34] \\
\hline 1414 & asymmetric $\mathrm{C}-\mathrm{H}$ bending & [35] \\
\hline 2965 & asymmetric $\mathrm{C}-\mathrm{H}$ stretching & [35] \\
\hline 2905 & symmetric $\mathrm{C}-\mathrm{H}$ stretching & [35] \\
\hline \multicolumn{3}{|c|}{$\mathrm{Si}-\mathrm{H}$} \\
\hline 2158 & Si-H stretching & [35] \\
\hline 912 & Si-H bending & [Oנ] \\
\hline \multicolumn{3}{|c|}{$\mathrm{Si}-\mathrm{O}-\mathrm{Si}$} \\
\hline 1023 & asymmetric in-Si-O-Si stretching & [25] \\
\hline
\end{tabular}

\subsection{Thermal Behaviour by TG-FTIR Analysis}

Thermogravimetric analyses under nitrogen revealed a different thermal behaviour of bulk PDMS and 3D porous PDMS foam, also highlighting various changes induced by the washing process, which were not apparent from the FTIR analysis.

Figure 3 shows the TG and DTG curves of bulk PDMS (Figure 3a,b) and 3D porous PDMS foam (Figure $3 \mathrm{c}, \mathrm{d}$ ) under $\mathrm{N}_{2}$, before and after washing in ethanol. 
(a)

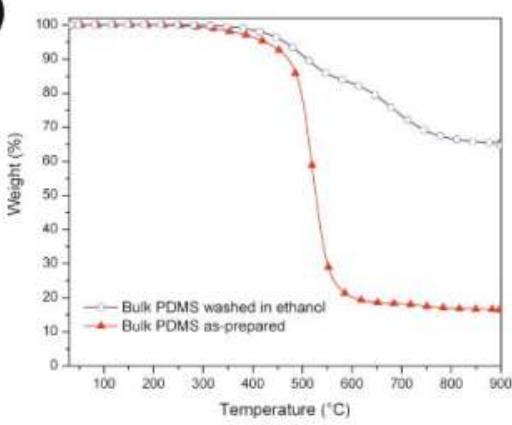

(c)

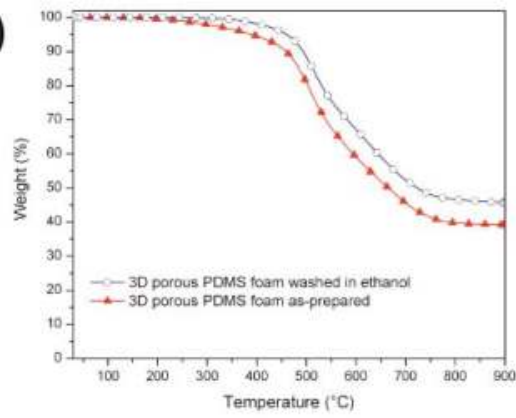

(b)

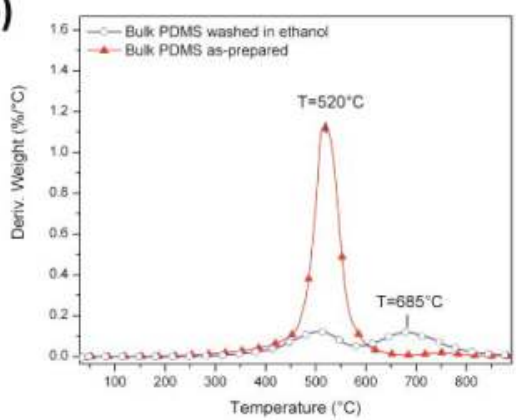

(d)

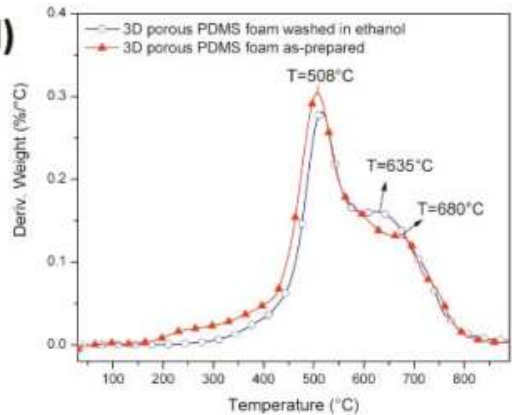

Figure 3. TG and DTG curves under $\mathrm{N}_{2}$ of bulk PDMS $(\mathbf{a}, \mathbf{b})$ and 3D porous PDMS foam $(\mathbf{c}, \mathbf{d})$ before and after washing in ethanol.

The as-prepared bulk PDMS showed the typical thermal degradation curve of PDMS [28], with a single sharp mass loss of almost $80 \%$ in the temperature range $400-600{ }^{\circ} \mathrm{C}$ and a maximum DTG at $520^{\circ} \mathrm{C}$ (Figure 3a,b). The as-prepared 3D porous PDMS foam showed a broader mass loss in the temperature range $300-750{ }^{\circ} \mathrm{C}$, with two overlapping steps with maxima at almost 510 and $680^{\circ} \mathrm{C}$.

Washing (both in ethanol and in hexane) produced an increase in the thermal stability of both bulk PDMS and 3D porous PDMS foam, but affected the thermal degradation behaviour of bulk PDMS more than that of 3D porous PDMS foam. The starting temperature of the thermal degradation of both bulk PDMS and 3D porous PDMS foam increased from 300 to $400{ }^{\circ} \mathrm{C}$ after washing, confirming the removal of low molecular weight oligomers and unreacted reagents from the materials. Above $400{ }^{\circ} \mathrm{C}$, the thermal degradation curves of 3D porous PDMS foam before and after washing were very similar, in contrast to those of bulk PDMS that are significantly different, with the curve measured after washing becoming more similar to that of $3 \mathrm{D}$ porous PDMS foam.

The mass loss spread over the temperature range $400-750^{\circ} \mathrm{C}$, revealing two overlapping steps with a maximum at almost 510 and $680^{\circ} \mathrm{C}$, respectively. The mass loss percentage and residual mass of bulk PDMS after washing presented a high variability (around 15\%) ascribed to the intrinsic bulk polymerization approach (yielding a more nonhomogeneous material). The PDMS thermal degradation mechanism proceeds via a depolymerization pathway occurring with two different mechanisms: "unzip degradation" and "rearrangement degradation" [28,29,36]. Unzip degradation generates cyclic siloxanes of different dimensions and occurs at about $400-500{ }^{\circ} \mathrm{C}$. Rearrangement degradation occurs at above $500{ }^{\circ} \mathrm{C}$ by heterolytic cleavage and the rearrangement of the $\mathrm{Si}-\mathrm{O}-\mathrm{Si}$ bond in the main chain and generates low molecular weight species and cyclic siloxanes. At $800^{\circ} \mathrm{C}$ under inert atmosphere, a black residue ascribed to silicon carbide or silicon oxycarbide is formed [28]. Methane $\left(\mathrm{CH}_{4}\right)$ is the second main byproduct generated by unzip thermal degradation through 
the homolytic $\mathrm{Si}-\mathrm{CH}_{3}$ bond scission followed by hydrogen abstraction $[29,37]$. The production of cyclic siloxanes and the corresponding residues are strongly affected by the cross-linking degree of the starting material and by the further cross-linking reaction occurring in the material during the thermogravimetric measurements $[28,29,36]$.

To further investigate the thermal decomposition mechanism of both the bulk and porous PDMS materials, the gaseous species evolved during their thermal degradation were analysed by evolved gas analysis (EGA) with FTIR spectroscopy (TG/ FTIR). As an example, Figure 4 shows the FTIR spectra of the gases evolved recorded at 505 and $685^{\circ} \mathrm{C}$ for bulk PDMS and at 515 and $635^{\circ} \mathrm{C}$ for the 3D porous PDMS foam after cleaning with ethanol. The FTIR spectra of the main degradation step recorded for the as-prepared bulk PDMS and the as-prepared 3D porous PDMS foam are reported in Figure S3. The main compounds identified in the evolved gas during the thermal decomposition of all the tested PDMS-based materials were linear and cyclic siloxane oligomers. The cyclic siloxane oligomers showed two main chemical groups: (1) $-\mathrm{Si}-\mathrm{CH}_{3}$ (FTIR wavenumber assignments $\mathrm{cm}^{-1}$ : 813 (Si-C stretching), 1265 (1260 symmetric $\mathrm{C}-\mathrm{H}$ bending [34]), 1412 (1414 asymmetric $\mathrm{C}-\mathrm{H}$ bending, [35]), 2967 (2965 asymmetric C-H stretching, [35]), 2904 (2905 symmetric C-H stretching, [35])) and, (2) -Si-O-Si- (FTIR wavenumber assignments $\mathrm{cm}^{-1}$ : 1027, 1085 due to the asymmetric $-\mathrm{Si}-\mathrm{O}-\mathrm{Si}$ stretching, [35]). Methane was the second chemical species detected (-C-H with FTIR wavenumber assignments of 1303 and $\left.3015 \mathrm{~cm}^{-1},[29,36]\right)$.
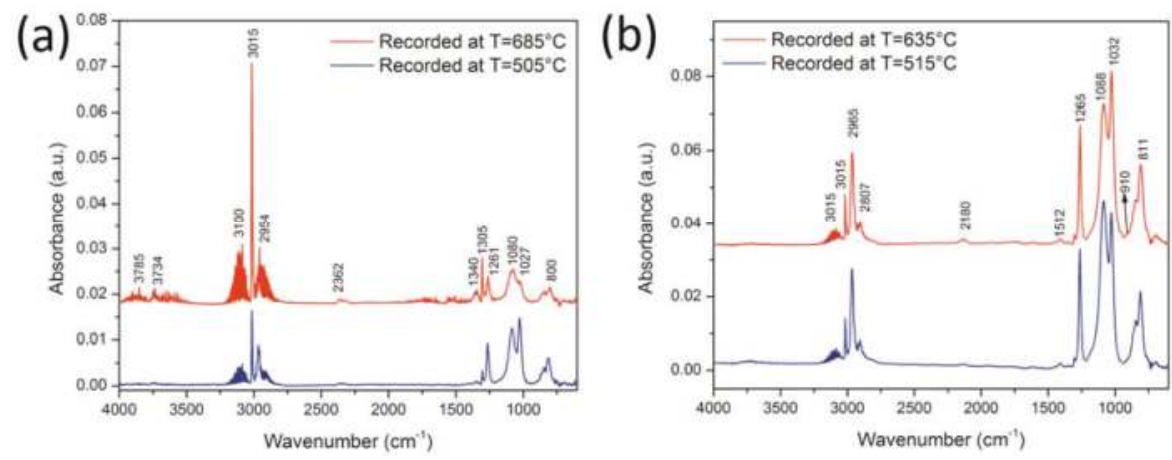

Figure 4. FTIR spectra of evolved gas from (a) bulk PDMS and (b) 3D porous PDMS foam recorded under $\mathrm{N}_{2}$ flow at the two main thermal decomposition steps. Bulk PDMS and 3D porous PDMS foam samples correspond to the ethanol cleaning samples.

Figure 5 shows the evolution profiles with the temperature of the main gaseous products (cyclic siloxanes and methane) obtained by monitoring their strongest IR bands for porous PDMS foam before (a) and after (b) washing with ethanol. The curves revealed in both cases two evolution bands of cyclic siloxane compounds, one below $500{ }^{\circ} \mathrm{C}$ and the other above $500{ }^{\circ} \mathrm{C}$ accounting for both the unzip and rearrangement polymer degradation (see Figure 5). The evolution profiles of cyclic siloxanes for bulk PDMS highlighted a single evolution band, at a temperature below $500{ }^{\circ} \mathrm{C}$, of these compounds for unwashed PDMS and two evolutions after washing. This suggests that the "rearrangement degradation" mechanism started to take place mainly after washing bulk PDMS, see Figure 6.

The $\mathrm{CH}_{4}$ evolution was present throughout the degradation time of the tested PDMS materials, though it was predominant at the end of the depolymerization, as the last event of the silicon carbide formation.

In summary, thermogravimetric data and FTIR analyses of the evolved gases suggested that porous PDMS foam is more homogeneous in terms of cross linking and more chemically stable than PDMS bulk. 


\section{(a) 3D porous PDMS foam as-prepared}

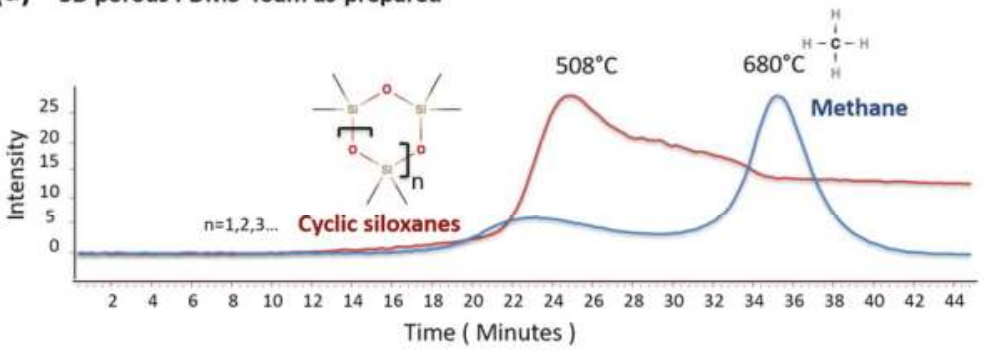

(b) 3D porous PDMS foam washed in ethanol

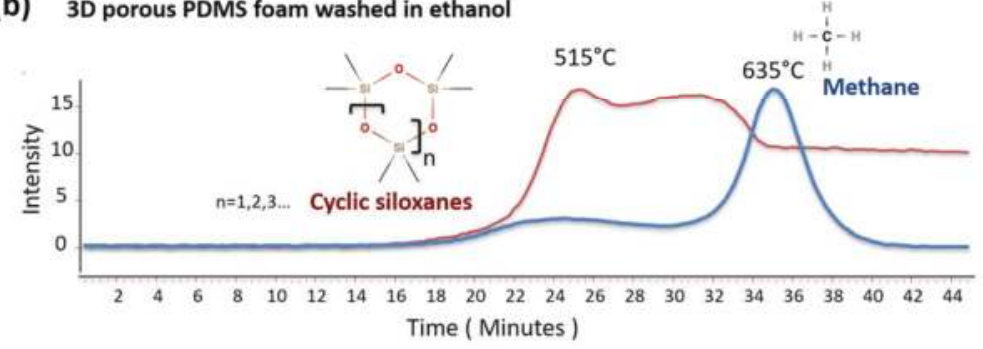

Figure 5. Evolution profiles of $\mathrm{CH}_{4}$ and cyclic siloxane compounds from the TG/FTIR of 3D porous PDMS foam thermal decomposition at $20^{\circ} \mathrm{C}$ min under $\mathrm{N}_{2}$.

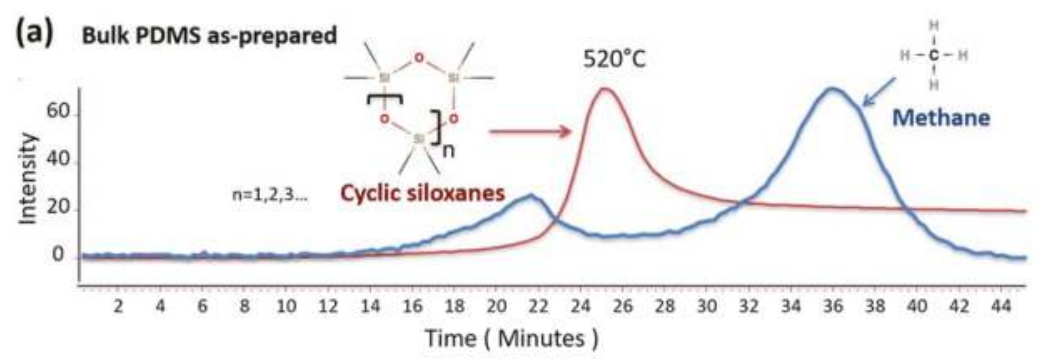

\section{(b) Bulk PDMS washed in ethanol}

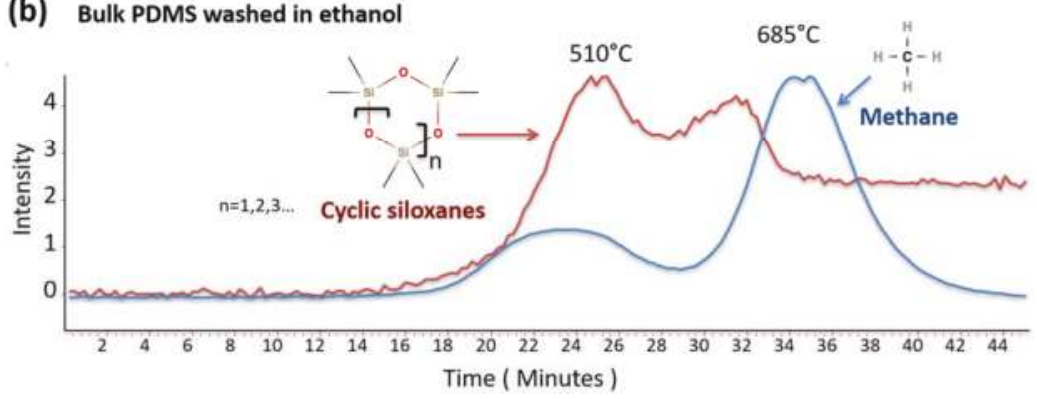

Figure 6. Evolution profiles of $\mathrm{CH}_{4}$ and cyclic siloxane compounds from the TG/FTIR of bulk PDMS thermal decomposition at $20^{\circ} \mathrm{C}$ min under $\mathrm{N}_{2}$. 


\section{Conclusions}

Given the increasing interest in porous PDMS foam in the field of sensors, we investigated the chemical composition and thermal degradation of a macroporous PDMS foam obtained using a sugar templating fabrication approach and to find a cleaning procedure based on PDMS washing with solvents to achieve a homogeneous porous PDMS material both in terms of chemical and physical properties. The ATR-FTIR spectra of the washing solvents showed that small oligomers of a PDMS base precursor and curing agent were completely removed from both porous and bulk PDMS. The latter was used as reference, after $72 \mathrm{~h}$ of soaking both in hexane and ethanol. Mass losses of $4.3 \% \pm 0.1 \%$ (hexane) and 3.7\% $\pm 0.3 \%$ (ethanol) for PDMS bulk and of 3.8\% $\pm 0.3 \%$ (hexane) and $3.4 \% \pm 0.8 \%$ (ethanol) for PDMS foam were registered after the first purification step, and no significant differences between the two solvents (i.e., hexane or ethanol) were observed.

The structural analysis by ATR-FTIR spectroscopy of both bulk PDMS and porous PDMS foam at the different steps in the cleaning procedure highlighted that no significant structural changes, with respect to the as-prepared materials, were induced by soaking and washing the PDMS in the tested solvents.

Interestingly, the thermogravimetric analyses under nitrogen gas revealed a different thermal behaviour between bulk PDMS and porous PDMS foam, which was also influenced by the washing process, especially for bulk PDMS. An increase in the thermal stability of both bulk PDMS and porous PDMS foam was apparent after the washing process, which also caused a modification of the thermal degradation behaviour of both the PDMS materials. In fact, the starting temperature of the thermal degradation of both bulk PDMS and 3D porous PDMS foam increased from 300 to $400{ }^{\circ} \mathrm{C}$, confirming the removal by washing of low molecular weight oligomers and unreacted reagents from the materials. Above $400{ }^{\circ} \mathrm{C}$, the thermal degradation curve of bulk PDMS changed significantly, becoming more similar to that of porous PDMS foam. The evolution profiles of the main gaseous products (cyclic siloxane and methane) obtained by monitoring their strongest IR bands over time for porous PDMS foam, revealed two evolutions of cyclic siloxane compounds. These consisted of one below $500{ }^{\circ} \mathrm{C}$ and the other that accounted for both the unzip and rearrangement polymer degradation.

In summary, thermogravimetric data highlighted a modification in the reactive pathway of PDMS materials due to a cleaning procedure. This information, which was not apparent from spectroscopic or morphological studies, would be very useful for planning the use of such complex and very reactive systems.

Supplementary Materials: The following are available online at http://www.mdpi.com/2073-4360/10/6/616/s1, Figure S1: ATR-FTIR spectra of bulk PDMS, 3D-porous PDMS foam, PDMS-curing agent and PDMS-base backbone precursors, Figure S2: ATR-FTIR spectra of bulk PDMS (a) and, 3D porous PDMS foam (b) after the materials were submitted to several steps of soaking and washing in hexane and ethanol. ATR-FTIR spectra were recorded after the materials were dried at $70{ }^{\circ} \mathrm{C}$ per $4 \mathrm{~h}$, Figure S3: FTIR spectra of evolved gas from (a) bulk PDMS and (b) 3D porous PDMS foam recorded at $T=520^{\circ} \mathrm{C}$ and $T=508^{\circ} \mathrm{C}$ under $\mathrm{N}_{2}$ flow at the main thermal decomposition step, respectively. Bulk PDMS and 3D porous PDMS foam samples correspond to as made samples, Video S1: 3D porous PDMS foam microCT $X$ ray reconstruction.

Author Contributions: Conceptualization: G.B., C.D. and M.R.T.; Sample preparation: R.I.; Data curation: J.G.-R. and C.D.; Funding acquisition: G.B. and M.R.T.; Investigation: J.G.-R., R.I., G.B., C.D. and M.R.T.; Supervision: M.R.T.; Writing—original draft: J.G.-R., R.I. and C.D.; Writing—review \& editing: G.B., C.D. and M.R.T.

Funding: This research was funded by University of Pisa: grant number PRA_2017_17.

Conflicts of Interest: The authors declare no conflict of interest.

\section{References}

1. Somasundaran, P.; Mehta, S.C.; Purohit, P. Silicone emulsions. Adv. Colloid Interface Sci. 2006, 128-130, 103-109. [CrossRef] [PubMed]

2. Eduok, U.; Faye, O.; Szpunar, J. Recent developments and applications of protective silicone coatings: A review of PDMS functional materials. Prog. Org. Coat. 2017, 111, 124-163. [CrossRef] 
3. Ammar, S.; Ramesh, K.; Vengadaesvaran, B.; Ramesh, S.; Arof, A.K. Amelioration of anticorrosion and hydrophobic properties of epoxy/PDMS composite coatings containing nano ZnO particles. Prog. Org. Coat. 2016, 92, 54-65. [CrossRef]

4. Li, J.; Zhao, Y.; Hu, J.; Shu, L.; Shi, X. Anti-icing performance of a superhydrophobic PDMS/modified nano-silica hybrid coating for insulators. J. Adhes. Sci. Technol. 2012, 26, 665-679. [CrossRef]

5. Kapridaki, C.; Maravelaki-Kalaitzaki, P. $\mathrm{TiO}_{2}-\mathrm{SiO}_{2}-\mathrm{PDMS}$ nano-composite hydrophobic coating with self-cleaning properties for marble protection. Prog. Org. Coat. 2013, 76, 400-410. [CrossRef]

6. Jagdale, P.; Salimpour, S.; Islam, M.H.; Cuttica, F.; Hernandez, F.C.R.; Tagliaferro, A.; Frache, A. Flame Retardant Effect of Nano Fillers on Polydimethylsiloxane Composites. J. Nanosci. Nanotechnol. 2018, 18, 1468-1473. [CrossRef] [PubMed]

7. Kim, Y.D.; Dordick, J.S.; Clark, D.S. Siloxane-based biocatalytic films and paints for use as reactive coatings. Biotechnol. Bioeng. 2001, 72, 475-482. [CrossRef]

8. Dunn, K.W.; Hall, P.N.; Khoo, C.T.K. Breast implant materials: Sense and safety. Br. J. Plast. Surg. 1992, 45, 315-321. [CrossRef]

9. Mattei, G.; Magliaro, C.; Giusti, S.; Ramachandran, S.D.; Heinz, S.; Braspenning, J.; Ahluwalia, A. On the adhesion-cohesion balance and oxygen consumption characteristics of liver organoids. PLoS ONE 2017, 12, e0173206. [CrossRef] [PubMed]

10. Chen, J.; Liu, T.; Tsou, H.; Ting, Y.; Tseng, Y. Biopolymer brushes grown on PDMS contact lenses by in situ atmospheric plasma-induced polymerization. J. Polym. Res. 2017, 24, 69. [CrossRef]

11. Srisa-Art, M.; Noblitt, S.D.; Krummel, A.T.; Henry, C.S. IR-Compatible PDMS microfluidic devices for monitoring of enzyme kinetics. Anal. Chim. Acta 2018, 1021, 95-102. [CrossRef] [PubMed]

12. Xia, Y.; McClelland, J.J.; Gupta, R.; Qin, D.; Zhao, X.M.; Sohn, L.L.; Celotta, R.J.; Whitesides, G.M. Replica molding using polymeric materials: A practical step toward nanomanufacturing. Adv. Mater. 1997, 9, 147-149. [CrossRef]

13. Surdo, S.; Diaspro, A.; Duocastella, M. Microlens fabrication by replica molding of frozen laser-printed droplets. Appl. Surf. Sci. 2017, 418, 554-558. [CrossRef]

14. Zhao, X.; Li, L.; Li, B.; Zhang, J.; Wang, A. Durable superhydrophobic/superoleophilic PDMS sponges and their applications in selective oil absorption and in plugging oil leakages. J. Mater. Chem. A 2014, 2, 18281-18287. [CrossRef]

15. Choi, S.J.; Kwon, T.H.; Im, H.; Moon, D.I.; Baek, D.J.; Seol, M.L.; Duarte, J.P.; Choi, Y.K. A polydimethylsiloxane (PDMS) sponge for the selective absorption of oil from water. ACS Appl. Mater. Interfaces 2011, 3, 4552-4556. [CrossRef] [PubMed]

16. Zhang, A.; Chen, M.; Du, C.; Guo, H.; Bai, H.; Li, L. Poly(dimethylsiloxane) oil absorbent with a three-dimensionally interconnected porous structure and swellable skeleton. ACS Appl. Mater. Interfaces 2013, 5, 10201-10206. [CrossRef] [PubMed]

17. Yu, C.; Yu, C.; Cui, L.; Song, Z.; Zhao, X.; Ma, Y.; Jiang, L. Facile Preparation of the Porous PDMS Oil-Absorbent for Oil/Water Separation. Adv. Mater. Interfaces 2017, 4, 1600862. [CrossRef]

18. Li, J.; Liu, X.; Crook, J.M.; Wallace, G.G. Development of a porous 3D graphene-PDMS scaffold for improved osseointegration. Colloids Surf. B Biointerfaces 2017, 159, 386-393. [CrossRef] [PubMed]

19. Zhou, T.; Yang, J.; Zhu, D.; Zheng, J.; Handschuh-Wang, S.; Zhou, X.; Zhang, J.; Liu, Y.; Liu, Z.; He, C.; et al. Hydrophilic Sponges for Leaf-Inspired Continuous Pumping of Liquids. Adv. Sci. 2017, 4, 1700028. [CrossRef] [PubMed]

20. Yang, W.; Nam, Y.G.; Lee, B.K.; Han, K.; Kwon, T.H.; Kim, D.S. Fabrication of a hydrophilic poly (dimethylsiloxane) microporous structure and its application to portable microfluidic pump. Jpn. J. Appl. Phys. 2010, 49, 06GM01. [CrossRef]

21. Cha, K.J.; Kim, D.S. A portable pressure pump for microfluidic lab-on-a-chip systems using a porous polydimethylsiloxane (PDMS) sponge. Biomed. Microdevices 2011, 13, 877-883. [CrossRef] [PubMed]

22. Iglio, R.; Mariani, S.; Robbiano, V.; Strambini, L.; Barillaro, G. Flexible Polydimethylsiloxane Foams Decorated with Multiwalled Carbon Nanotubes Enable Unprecedented Detection of Ultralow Strain and Pressure Coupled with a Large Working Range. ACS Appl. Mater. Interfaces 2018, 10, 13877-13885. [CrossRef] [PubMed] 
23. Duan, S.; Yang, K.; Wang, Z.; Chen, M.; Zhang, L.; Zhang, H.; Li, C. Fabrication of Highly Stretchable Conductors Based on 3D Printed Porous Poly(dimethylsiloxane) and Conductive Carbon Nanotubes/ Graphene Network. ACS Appl. Mater. Interfaces 2016, 8, 2187-2192. [CrossRef] [PubMed]

24. Chen, M.; Zhang, L.; Duan, S.; Jing, S.; Jiang, H.; Li, C. Highly stretchable conductors integrated with a conductive carbon nanotube/graphene network and 3D porous poly (dimethylsiloxane). Adv. Funct. Mater. 2014, 24, 7548-7556. [CrossRef]

25. Silva, F.A.; Chagas-Silva, F.A.; Florenzano, F.H.; Pissetti, F.L. Poly(dimethylsiloxane) and Poly [vinyltrimethoxysilane-co-2-(dimethylamino) ethyl methacrylate] Based Cross-Linked Organic-Inorganic Hybrid Adsorbent for Copper(II) Removal from Aqueous Solutions. J. Braz. Chem. Soc. 2016, 27, 2181-2191. [CrossRef]

26. Krajnc, M.; Šebenik, U. Kinetic Study of Thermal Degradation of Polydimethylsiloxane: The Effect of Molecular Weight on Thermal Stability in Inert Atmosphere. Polym. Sci. 2017, 3, 6-11. [CrossRef]

27. Thomas, T.H.; Kendrick, T.C. Thermal analysis of polydimethylsiloxanes. I. Thermal degradation in controlled atmospheres. J. Polym. Sci. Part B Polym. Phys. 1969, 7, 537-549. [CrossRef]

28. Camino, C.; Lomakin, S.M.; Lazzari, M. Polydimethylsiloxane thermal degradation Part 1. Kinetic aspects, Polymer (Guildf.) 2001, 42, 2395-2402. [CrossRef]

29. Camino, G.; Lomakin, S.M.; Lageard, M. Thermal polydimethylsiloxane degradation. Part 2. The degradation mechanisms. Polymer (Guildf.) 2002, 43, 2011-2015. [CrossRef]

30. Iglio, R.; Mariani, S.; Strambini, L.M.; Baribaro, G. Macroporous PDMS foam decorated with carbon nanotubes for conductometric pressure and strain sensors. In Proceedings of the IEEE SENSORS, Glasgow, UK, 29 October-1 November 2017; pp. 1-3. [CrossRef]

31. Herz, J.; Munch, J.P.; Candau, S. Experimental investigation of the role of trapped entanglements in swollen polydimethylsiloxane networks. J. Macromol. Sci. Part B 1980, 18, 267-279. [CrossRef]

32. Rana, D.; Kim, H.L.; Kwag, H.; Choe, S. Hybrid blends of similar ethylene 1-octene copolymers. Polymer (Guildf.) 2000, 41, 7067-7082. [CrossRef]

33. Rana, D.; Kim, H.L.; Kwag, H.; Rhee, J.; Cho, K.; Woo, T.; Lee, B.H.; Choe, S. Blends of Ethylene 1-Octene Copolymer Synthesized by Ziegler-Natta and Metallocene Catalysts. I. Thermal and Mechanical Properties. J. Appl. Polym. Sci. 2000, 76, 1950-1964. [CrossRef]

34. Johnson, L.M.; Gao, L.; Shields, C.W., IV; Smith, M.; Efimenko, K.; Cushing, K.; Genzer, J.; López, G.P. Elastomeric microparticles for acoustic mediated bioseparations. J. Nanobiotechnol. 2013, 11, 22. [CrossRef] [PubMed]

35. Gaboury, S.R.; Urban, M.W. Quantitative analysis of the Si-H groups formed on poly(dimethylsiloxane) surfaces: An ATR FTi.r. approach. Polymer (Guildf.) 1992, 33, 5085-5089. [CrossRef]

36. Sun, J.T.; Huang, Y.D.; Cao, H.L.; Gong, G.F. Effects of ambient-temperature curing agents on the thermal stability of poly (methylphenylsiloxane). Polym. Degrad. Stab. 2004, 85, 725-731. [CrossRef]

37. Fang, W.; Zeng, X.; Lai, X.; Li, H.; Chen, W.; Zhang, Y. Thermal degradation mechanism of addition-cure liquid silicone rubber with urea-containing silane. Thermochim. Acta 2015, 605, 28-36. [CrossRef]

(c) 2018 by the authors. Licensee MDPI, Basel, Switzerland. This article is an open access article distributed under the terms and conditions of the Creative Commons Attribution (CC BY) license (http:/ / creativecommons.org/licenses/by/4.0/). 
Article

\title{
Sol-Gel Synthesis, Spectroscopic and Thermal Behavior Study of $\mathrm{SiO}_{2} / \mathrm{PEG}$ Composites Containing Different Amount of Chlorogenic Acid
}

\author{
Michelina Catauro ${ }^{1, *}$, Elisabetta Tranquillo ${ }^{1,2}$, Roberta Risoluti ${ }^{3}$ and Stefano Vecchio Ciprioti ${ }^{4}$ \\ 1 Department of Engineering, University of Campania “Luigi Vanvitelli”, Via Roma 29, I-81031 Aversa, Italy; \\ elisabetta.tranquillo@unicampania.it \\ 2 Department of Biochemistry, Biophysics and General Pathology, Medical School, University of Campania \\ "Luigi Vanvitelli", Via L. De Crecchio 7, 80138 Naples, Italy \\ 3 Department of Chemistry_-“Sapienza”, University of Rome, p.le A.Moro 5, 00185 Rome, Italy; \\ roberta.risoluti@uniroma1.it \\ 4 Department S.B.A.I., Sapienza University of Rome, Via Del Castro Laurenziano 7, 00185 Rome, Italy; \\ stefano.vecchio@uniroma1.it \\ * Correspondence: michelina.catauro@unicampania.it
}

Received: 31 May 2018; Accepted: 16 June 2018; Published: 19 June 2018

\begin{abstract}
In this work, new phenol-based materials have been synthesized by the sol-gel method, in which different amounts of the phenolic antioxidant chlorogenic acid (CGA) (from $5 \mathrm{wt} \%$ to $20 \mathrm{wt} \%$ ) were embedded in two different silica matrices: pure silica and silica-based hybrids materials, containing $50 \mathrm{wt} \%$ of polyethylene glycol (PEG). The incorporation of CGA in different sol-gel matrices might protect them from degradation, which could cause the loss of their properties. The two series of materials were chemically characterized by Fourier transform infrared (FTIR) spectroscopy. In addition, the thermal behavior of both series of materials containing CGA was studied by thermogravimetry under both air and inert $\mathrm{N}_{2}$ flowing gas atmosphere. The bioactivity was evaluated by soaking the synthesized hybrids in a simulated body fluid, showing that the bioactivity of the silica matrix is not modified by the presence of PEG and CGA.
\end{abstract}

Keywords: sol-gel; hybrids; chlorogenic acid; bioactivity; FTIR; TG

\section{Introduction}

Biomaterial implants are clinically used in a variety of applications, and their interaction with cells or tissue is important for the patient health and quality of life. They should act as artificial replacements, since the primary requirement of any material is its biocompatibility inside the human body [1]. Immediately following implantation, proteins and other biomolecules that are present in the blood plasma and biological fluids rapidly adsorb on the surface of biomaterials, leading to structural changes and different biological response [2]. Adverse host responses to implanted biomedical devices could induce a cascade of biological events that cause implant failure [3], which is due to many factors, with infection and inflammations among the most important of them. Although inflammation recruits native cells for remodeling and regenerating the damaged tissue, persistent and inflammatory stimuli significantly interfere with the implant function, and often result in device failure [4]. During the inflammatory processes, the production of high amounts of reactive oxygen species (ROS) and $\mathrm{H}_{2} \mathrm{O}_{2}$ occurs. These inflammatory events of implant integration are necessary at the early stages for efficient wound healing, but a persistent oxidative stress might be the main cause of failure in orthopedic implants [5]. To overcome this problem, orally administered drugs have typically been used to control chronic tissue inflammation, but may not achieve adequate local concentrations. Therefore, controlled 
local release systems may provide the desired constant drug concentrations at the delivery site and a potential reduction of deleterious side effects $[4,6]$.

In this work, new silica and silica/polyethylene glycol composites containing different percentages of chlorogenic acid, which is widely found in fruits and vegetables, were synthesized, characterized, and proposed as controlled drug delivery systems.

The synthesis procedure adopted was the sol-gel process, which is a technique widely used to make glasses and ceramics at low temperature. It is an interesting approach to prepare hybrid materials, because it takes place in solution, introducing easily the organic phases in an inorganic material, while preventing its degradation [7]. The reactions occurring during the sol-gel process are: (i) hydrolysis of alkoxide precursors to form a solution (sol phase) and (ii) condensation of the intermediate species that causes the formation of a 3D network (gel phase) [8]. This technique has been widely employed in the preparation of hybrid materials, composed by intimately distributed organic and inorganic phases.

It is reported in the literature that the sol-gel materials, in particular those with a silica matrix, have been investigated as carriers for different drugs $[9,10]$. In this work, two different organic compounds are incorporated in a silica matrix: polyethylene glycol (PEG) and chlorogenic acid (CGA). PEG is a versatile, biocompatible polymer that is mainly used in polymer-based materials for drug delivery [11]. It is recognized in many studies [12-14] that some of the most valuable biological properties of drugs, such as reducing toxicity and extending the circulation time of many drug nanocarriers, were improved by the addition of PEG into the material.

Chlorogenic acid is a member of the group of polyphenolic compounds that has many biological properties, including antibacterial, antihypertensive, antitumor, anti-inflammatory, and osteogenic potential [15-20]. The main purpose of incorporating CGA in the silica matrix is due to protecting the natural compound from adverse environmental conditions, such as the undesirable effects of light and oxygen, increasing the shelf life of the drug, and promoting a controlled delivery [21]. In particular, it is possible to protect the drug from degradation that causes the loss of its properties [22]. In fact, the decomposition products of CGA are widely recognized as undesirable compounds [23-27]. So, the knowledge of the decomposition behavior of CGA is extremely important in biomedical fields.

The aim of this study is the synthesis and characterization of $\mathrm{SiO}_{2} / \mathrm{CGA}$ and $\mathrm{SiO}_{2} / \mathrm{PEG} / \mathrm{CGA}$ hybrid materials. Fourier transform infrared (FTIR) spectroscopy was used, similar to what has been made in recent studies for silica-based materials [28,29], to evaluate the interactions among different components incorporated in the hybrid materials. Furthermore, the hybrids were soaked in a simulated body fluid (SBF) to evaluate their ability to stimulate the hydroxyapatite nucleation by FTIR.

Thermal analysis is recognized as a powerful tool to analyze the thermooxidative behavior of polymer composites [30-33]. The thermal behavior of silica/CGA and silica/PEG/CGA materials under both air and inert $\mathrm{N}_{2}$ flowing gas atmosphere was studied by thermogravimetry under both inert and oxidative flowing gas atmosphere to analyze the different thermal behavior due to the inert or oxidative environments.

\section{Materials and Methods}

\subsection{Sol-Gel Synthesis}

$\mathrm{SiO}_{2} / \mathrm{CGA}$ and $\mathrm{SiO}_{2} / \mathrm{PEG} / \mathrm{CGA}$ organic-inorganic hybrids materials were synthesized by the sol-gel method according to a procedure reported in a recent study [34]. A solution of tetraethyl orthosilicate (TEOS, reagent grade, 98\%, Sigma Aldrich, Steinheim am Albuch, Germany) was used as a precursor of the $\mathrm{SiO}_{2}$ inorganic matrix. TEOS, ethanol 99\% (Sigma Aldrich, Steinheim am Albuch, Germany), nitric acid (solution 65\%, Sigma Aldrich, Steinheim am Albuch, Germany), and water were added in a solution under stirring; nitric acid was used to promote the kinetics of hydrolysis and condensation reactions. The molar ratios among the reagents in the obtained solution are: $\mathrm{EtOH} / \mathrm{TEOS}=6.2, \mathrm{TEOS} / \mathrm{HNO}_{3}=1.7, \mathrm{H}_{2} \mathrm{O} / \mathrm{TEOS}=6$. 
The hybrid materials were obtained using ethanol solutions of CGA at different percentages ( 5 wt \%, 10 wt \%, 15 wt \%, 20 wt \%), while a high amount of polyethylene glycol (MW = 400, Sigma Aldrich) (PEG $50 \mathrm{wt} \%$ ) dissolved in ethanol was added to the pure silica matrix (to reach $50 \mathrm{wt} \%$ ) before preparing the $\mathrm{SiO}_{2} / \mathrm{PEG} / \mathrm{CGA}$ materials. The different prepared solutions were put at room temperature until the gel was obtained, and then left into an oven at $40^{\circ} \mathrm{C}$ to allow for the removal of the residue solvent, thus avoiding the thermal degradation of the drug. The flow chart of the sol-gel process is shown in Figure 1.

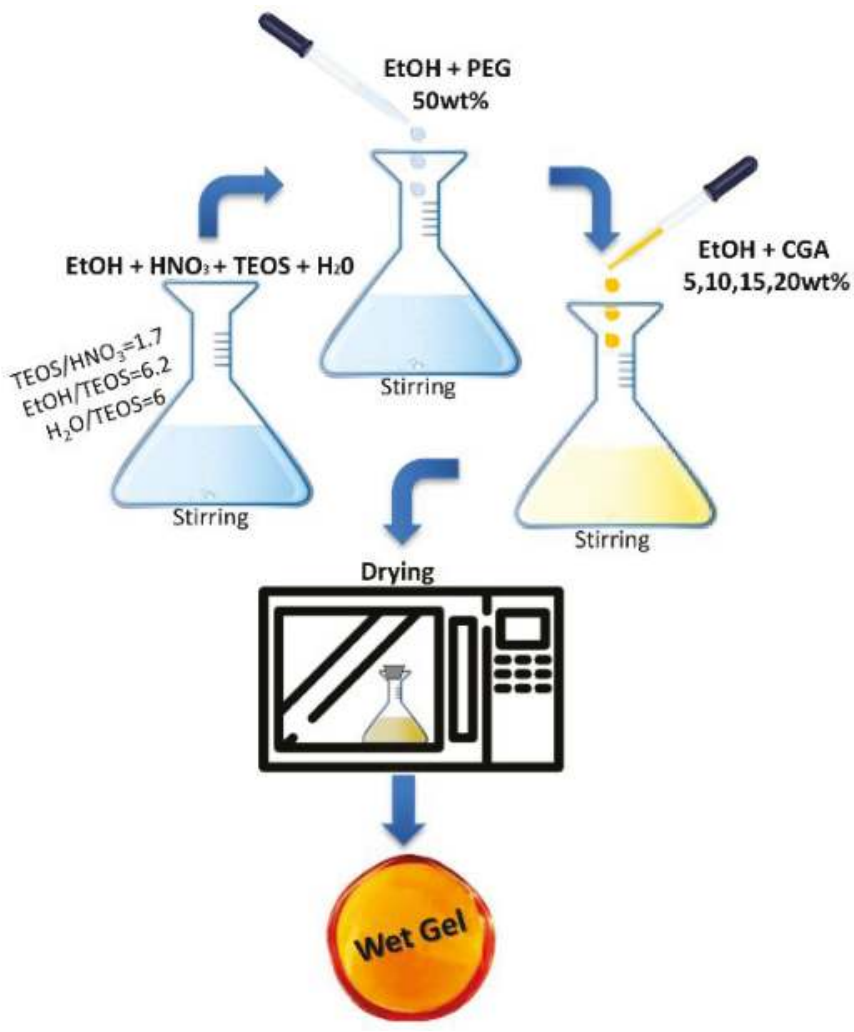

Figure 1. Flow chart of the sol-gel hybrid synthesis and the molar ratios between the reagents achieved in the sol.

\subsection{Materials Characterization}

FTIR spectroscopy was used to investigate the chemical composition of the materials and the interactions among their components. A Prestige 21 (Shimadzu, Kyoto, Japan) system, equipped with a DTGS KBr (deuterated triglycine sulfate with potassium bromide windows) detector allowed us to record transmittance spectra in the $400-4000 \mathrm{~cm}^{-1}$ region, with a resolution of $2 \mathrm{~cm}^{-1}$ (45 scans). Then, $2 \mathrm{mg}$ of sample powder, mixed with $198 \mathrm{mg}$ of $\mathrm{KBr}$, was compacted into discs under a pressure of 7 tons by using a hydraulic press (Specac, Orpington, UK). The FTIR spectra were elaborated by the Prestige software (IR solution).

Thermogravimetric (TG) analysis of all of the investigated samples was performed by a TGA7 Thermobalance (PerkinElmer, Waltham, MA, USA). Samples were placed into a platinum crucible, and the temperature was measured using a thermocouple directly connected to the crucible. 
The temperature was raised from $20{ }^{\circ} \mathrm{C}$ to $800{ }^{\circ} \mathrm{C}$ at a heating rate of $10{ }^{\circ} \mathrm{C} \mathrm{min}-1$, with the best resolution rate permitting to differentiate the contribution of all of the components. The TG analyses were performed both under an inert gas flux of nitrogen and oxidant atmosphere of air at $100 \mathrm{~mL}$ $\mathrm{min}^{-1}$. Temperature calibration was performed using the Curie-point transition of standard metals, as specified by the equipment recommendations. Each sample was analyzed in triplicate, and a high reproducibility of the resulting curves was observed.

\subsection{Bioactivity Test}

The in vitro apatite-forming ability test [35] was used to evaluate the bioactivity of the hybrid materials.

All of the hybrid materials were crushed to a powder using an agate mortar and soaked for seven, 14 and 21 days in a simulated body fluid (SBF) with ion concentrations nearly equal to those found in human blood plasma. In order to maintain the SBF solution temperature fixed at $37^{\circ} \mathrm{C}$, the samples were placed in polystyrene bottles in a water bath. As the ratio between the total surface area of the material exposed to SBF and its volume influences the reaction of hydroxyapatite nucleation, a constant ratio was maintained, as reported in the literature. Moreover, the SBF solution, in which the samples were soaked, was exchanged every two days to avoid depletion of the ionic species in the SBF due to the formation of biominerals. After each soaking period, the samples were removed from the SBF and air-dried in a desiccator. After $24 \mathrm{~h}$, they were subjected to FTIR analysis in order to evaluate the ability to form an apatite layer on their surfaces.

\section{Results and Discussion}

\subsection{FTIR Structural Characterization}

In order to evaluate the chemical structure of $\mathrm{SiO}_{2} / \mathrm{PEG} / \mathrm{CGA}$ hybrid materials, their FTIR spectra are displayed in Figure 2. The hybrids' spectra of materials were compared with pure PEG and pure silica to identify their interaction. In the $\mathrm{SiO}_{2}$ spectrum (curve f), all of the typical peaks of the silica sol-gel materials [36,37] are visible. In particular, the asymmetric and symmetric Si-O stretching vibrations are recorded at $1080 \mathrm{~cm}^{-1}$ with shoulders at $1200 \mathrm{~cm}^{-1}$ and at $800 \mathrm{~cm}^{-1}$. The peaks at $460 \mathrm{~cm}^{-1}$ and $960 \mathrm{~cm}^{-1}$ were attributed to the bending vibrations of $\mathrm{Si}-\mathrm{O}-\mathrm{Si}$ bonds and $\mathrm{Si}-\mathrm{OH}$ bond vibrations, respectively [38]. Furthermore, in the pure silica spectra, it is possible to observe the low intensity band at $580 \mathrm{~cm}^{-1}$ due to residual four-membered siloxane rings in the silica network $[36,37,39,40]$ and the sharp $\mathrm{N}-\mathrm{O}$ stretching band of residual nitrate anions at $1382 \mathrm{~cm}^{-1}$ [41] caused by the presence of residual $\mathrm{HNO}_{3}$, which was used as a catalyst in the synthesis procedure. The position and shape of the bands at $3445 \mathrm{~cm}^{-1}$ and $1640 \mathrm{~cm}^{-1}$ attributed to $-\mathrm{OH}$ stretching and bending vibrations in the hydration water suggest the presence of $\mathrm{H}$-bonded solvent molecules $\left(\mathrm{H}_{2} \mathrm{O}\right)$ and hydrogen bonded -OH groups attached to the $\mathrm{Si}$ atoms. The addition of high amounts of PEG $(50 \%)$ in the silica matrix allows observing, in the hybrid spectra (curve b-e), some peaks typical of the polymer. The bands at $2930-2870 \mathrm{~cm}^{-1}$ and $1454 \mathrm{~cm}^{-1}$ are due to PEG methylene $\mathrm{C}-\mathrm{H}$ stretching and bending, respectively [42]. The typical signals of the $C-C$ groups at $948 \mathrm{~cm}^{-1}$ are clearly visible, as well as the characteristic $\mathrm{C}-\mathrm{O}$ stretching band of alcohols at $1250 \mathrm{~cm}^{-1}[43,44]$. In the hybrid spectra, the change in the shape of the broad band at about $3400 \mathrm{~cm}^{-1}$, as well as the displacement of the $\mathrm{Si}-\mathrm{OH}$ band and the peak at $580 \mathrm{~cm}^{-1}$ at a lower wavenumber, may be due to the formation of hydrogen bonds [45] between the $-\mathrm{OH}$ groups of the inorganic phases and ethereal oxygen atoms (H-bond donors) or terminal -OH in the PEG chains. These results suggest that the presence of chlorogenic acid does not change the structure and interactions of $\mathrm{SiO}_{2} / \mathrm{PEG}$ hybrid materials.

Figure 3 shows the FTIR spectra of the hybrids with different percentages of $\left.\mathrm{CGA}_{(5,10,15,20 \mathrm{wt}} \%\right)$ compared with pure CGA (curve a), $\mathrm{SiO}_{2} / \mathrm{CGA}_{5 \mathrm{wt}} \%$, and $\mathrm{SiO}_{2} / \mathrm{CGA}_{20} \mathrm{wt} \%$ (curves b, g). The spectrum of pure CGA shows the stretching of $\mathrm{OH}$ groups at $3468 \mathrm{~cm}^{-1}$ and $3344 \mathrm{~cm}^{-1}$, whereas the $\mathrm{OH}$ bending of the phenol function is visible at $1382 \mathrm{~cm}^{-1}$ [34]. The latter band in the spectrum of 
$\mathrm{SiO}_{2} / \mathrm{PEG}_{50} \mathrm{wt} \% / \mathrm{CGA}_{5} \mathrm{wt} \%$ hybrid (curve c) is more intense than that related to the other hybrids containing the polymer. In particular, this peak $\left(1382 \mathrm{~cm}^{-1}\right)$ is visible also in the spectrum of $\mathrm{SiO}_{2} / \mathrm{CGA}_{5 \mathrm{wt}} \%$ (curve b), which is probably due to an interaction between the silica matrix and CGA: increasing the drug concentration caused a decrease of the peak intensity, because the drug at low concentrations is more embedded and linked within it by the formation of $-\mathrm{H}$ bonds. On the other hand, when a higher amount of CGA is added in pure silica (curve g) and in the silica/PEG matrix (curves d, e, f), a part of CGA cannot form H-bonds, because all of the hydroxyl groups of silica are already involved in $\mathrm{H}$-bonds.

The stretching $\mathrm{C}=\mathrm{O}$ vibration band is observed at $1726 \mathrm{~cm}^{-1}$ in the spectrum of pure CGA; instead, its displacement occurs in all of the hybrids' spectra, this effect can be explained by the establishment of $\mathrm{H}$-bonds with the $\mathrm{SiO}_{2}$ inorganic matrix [34]. The different shape and a broadening of the $\mathrm{SiO}_{2}$ strong band at $1080 \mathrm{~cm}^{-1}$, as well as a marked increase of the intensity of the shoulder at $1200 \mathrm{~cm}^{-1}$, is due to the presence in this spectral region of intense signals of the phenyl ring and $\mathrm{C}-\mathrm{O}-\mathrm{C}$ bonds in CGA (see curve a), and also to interactions with the polymer.

Therefore, all of the acquired spectra suggest that chlorogenic acid was embedded in the silica/PEG matrix, and all of the components are linked to each other by hydrogen bonds, which play a crucial role in the structure of hybrids.

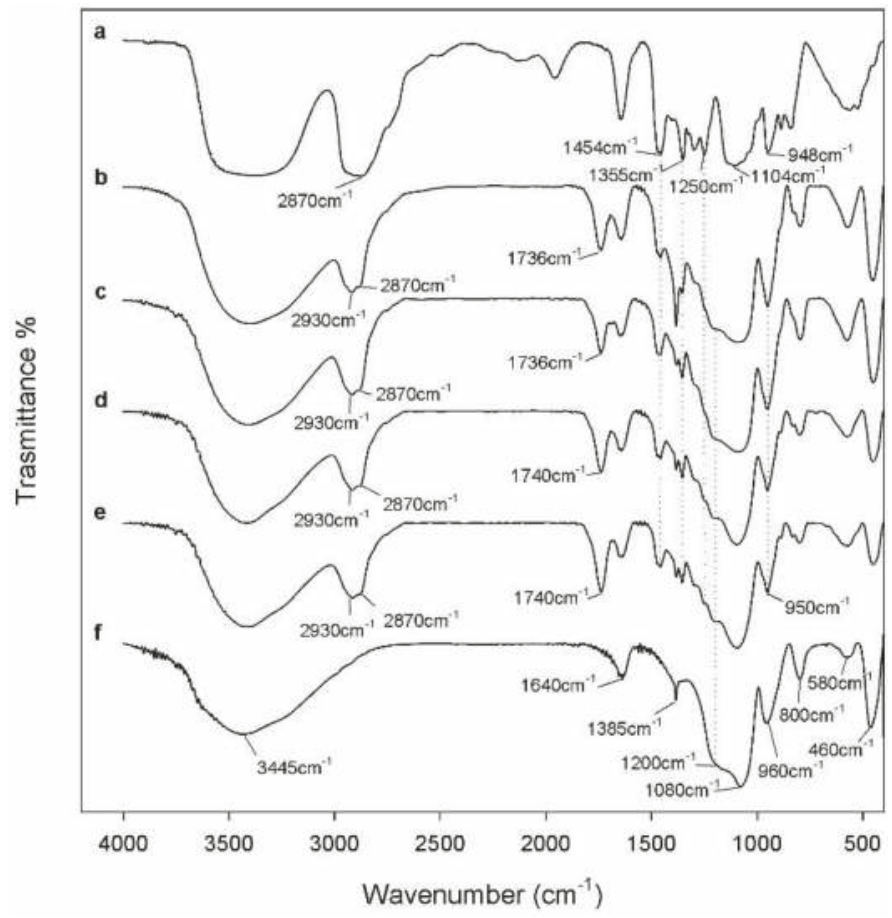

Figure 2. Representative Fourier transform infrared (FTIR) spectra of: (a) pure polyethylene glycol (PEG); (b) $\mathrm{SiO}_{2} / \mathrm{PEG}_{50 \mathrm{wt}} \% /$ chlorogenic acid (CGA) $5 \mathrm{wt} \%$; (c) $\mathrm{SiO}_{2} / \mathrm{PEG}_{50} \mathrm{wt} \% / \mathrm{CGA}_{10} \mathrm{wt} \%$;

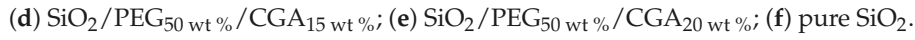




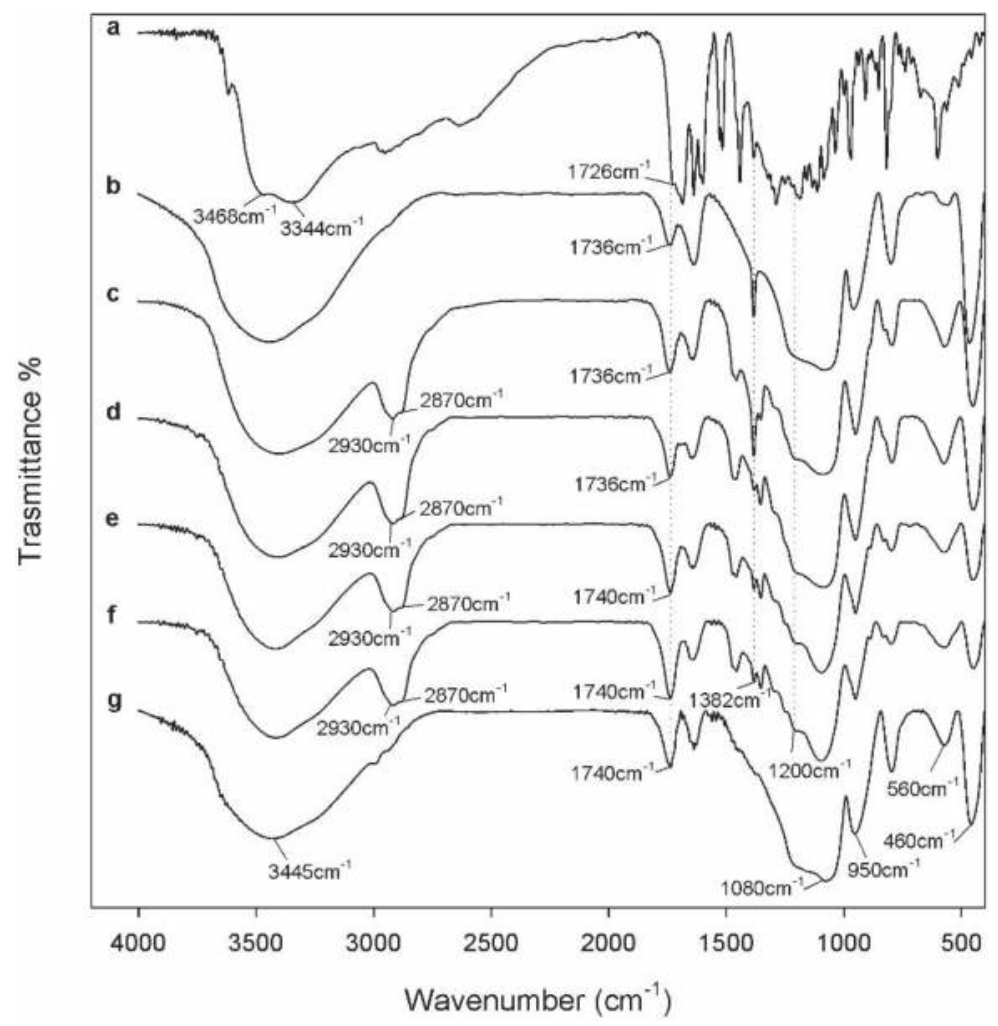

Figure 3. Representative FTIR spectra of: (a) pure CGA; (b) $\mathrm{SiO}_{2} / \mathrm{CGA}_{5 \mathrm{wt}} \%$; (c) $\mathrm{SiO}_{2} / \mathrm{PEG}_{50 \mathrm{wt}} \% / \mathrm{CGA}_{5 \mathrm{wt}} \%$; (d) $\mathrm{SiO}_{2} / \mathrm{PEG}_{50 \mathrm{wt}} \% / \mathrm{CGA}_{10 \mathrm{wt}} \%$; (e) $\mathrm{SiO}_{2} / \mathrm{PEG}_{50} \mathrm{wt} \% / \mathrm{CGA}_{15} \mathrm{wt} \%$; (f) $\mathrm{SiO}_{2} / \mathrm{PEG}_{50} \mathrm{wt} \% / \mathrm{CGA}_{20} \mathrm{wt} \%$; (g) $\mathrm{SiO}_{2} / \mathrm{CGA}_{20} \mathrm{wt} \%$.

\subsection{Thermal Behavior Study}

The thermal behavior of the two classes of hybrids under inert and oxidative atmosphere has been studied by recording TG experiments in flowing nitrogen and air at $10{ }^{\circ} \mathrm{C} \mathrm{min}-1$ (see the Materials and Methods Section). The corresponding TG curves of the $\mathrm{SiO}_{2} / \mathrm{CGA}$ and $\mathrm{SiO}_{2} / \mathrm{PEG}_{50} \mathrm{wt} \% / \mathrm{CGA}$ materials (with increasing content of CGA from $5 \mathrm{wt} \%$ to $20 \mathrm{wt} \%$ ) are reported in Figures 4 and 5, respectively. For comparison purposes, the TG curves of both pure silica and $\mathrm{SiO}_{2} / \mathrm{PEG}_{50} \mathrm{wt} \%$ carried out under a stream of Ar and recently published [46] are also reported in both plots $a$ of Figures 4 and 5 , respectively. 

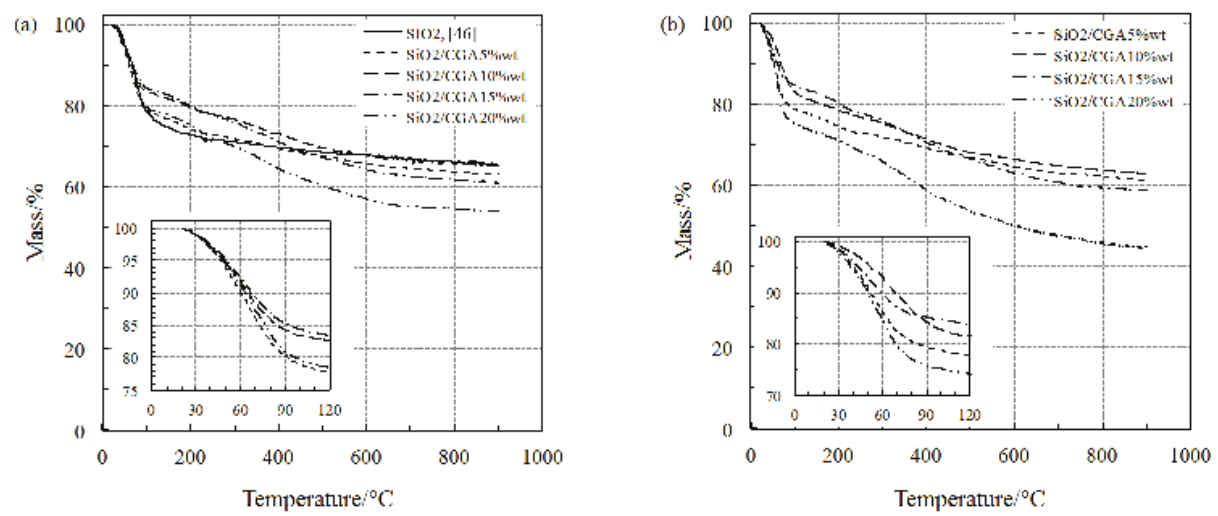

Figure 4. Thermogravimetric (TG) curves of pure $\mathrm{SiO}_{2}$ and $\mathrm{SiO}_{2} / \mathrm{CGA}$ hybrids at $10{ }^{\circ} \mathrm{C} \mathrm{min}-1$ under a flowing atmosphere of: nitrogen (a); air (b).
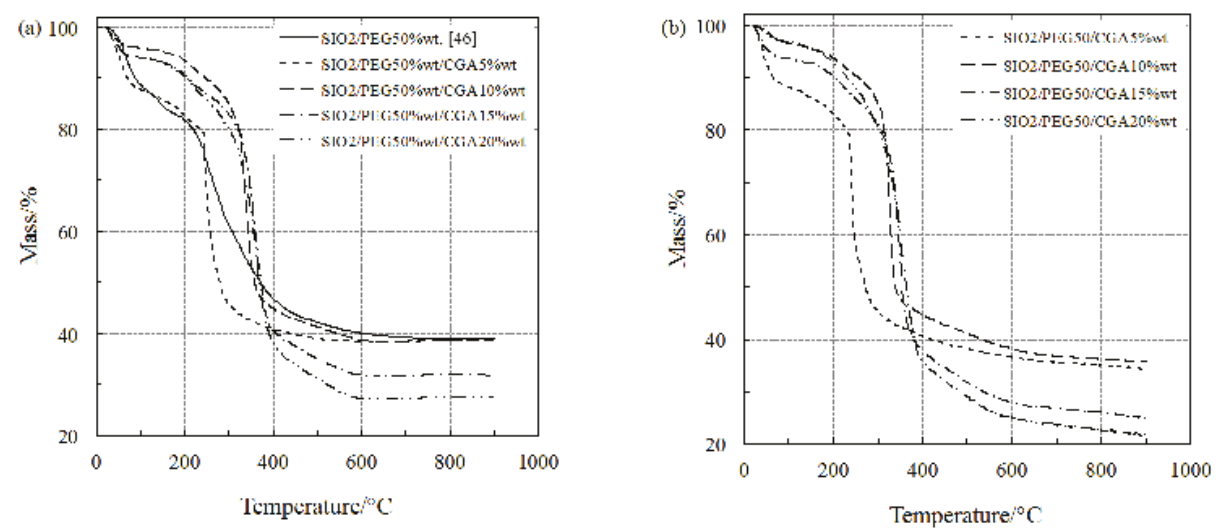

Figure 5. TG curves of $\mathrm{SiO}_{2} / \mathrm{PEG}$ and $\mathrm{SiO}_{2} / \mathrm{PEG} / \mathrm{CGA}$ hybrids at $10^{\circ} \mathrm{C} \mathrm{min}^{-1}$ under a flowing atmosphere of: nitrogen (a); air (b).

Under inert atmosphere, the $\mathrm{SiO}_{2} / \mathrm{CGA}$ hybrids undergo a first mass loss of up to $120{ }^{\circ} \mathrm{C}$ (Figure 4a) ascribed to dehydration, although the loss of residual ethanol used for the synthesis cannot be excluded. The thermal behavior of these materials in this temperature range is identical to that of pure silica [46], as is evident by the perfect superimposition of the TG curves. The water released by the CGA-poorest and CGA-richest hybrids $\left(\mathrm{SiO}_{2} / \mathrm{CGA}_{5 \mathrm{wt}} \%\right.$ and $\mathrm{SiO}_{2} / \mathrm{CGA}_{20} \mathrm{wt} \%$, respectively) when this process reaches its completion from $100{ }^{\circ} \mathrm{C}$ to $120^{\circ} \mathrm{C}$ (see the inner plot of Figure 4a) is about $9 \%$ higher than that of the other two. A high amount of weakly bonded water is removed from $\mathrm{SiO}_{2} / \mathrm{CGA}_{5 \mathrm{wt}} \%$, since only a limited number of $\mathrm{H}$-bonds is formed because of the poor content of CGA. On the other hand, $\mathrm{SiO}_{2} / \mathrm{CGA}_{20} \mathrm{wt} \%$ may retain a remarkable content of water and/or ethanol (removed by dehydration in the first step of the TG curve), because the higher the amount of CGA in the hybrid, the higher the number of formed H-bonds. Slight differences in the percentages of mass loss due to dehydration were observed when the same process takes place in air (Figure $4 \mathrm{~b}$ ), where the temperature range for dehydration shifts toward lower values as the amount of water increases (inner plot in Figure 4b). 
Starting from about $160{ }^{\circ} \mathrm{C}$, a second step of mass loss occurred in a wide temperature range in both inert and air atmosphere for all of the $\mathrm{SiO}_{2}$ /CGA hybrids. This loss was probably ascribable to the thermal degradation (pyrolysis) of CGA (plot of both Figure 4a,b, respectively) while pure $\mathrm{SiO}_{2}$ undergoes dehydroxylation [46]. It also corresponds to the slow elimination of water in a wide temperature range, which is caused by the condensation of surface hydroxyl groups.

As far as the multi-step thermal degradation of CGA is concerned, higher percentages of mass were recorded for materials treated in air with respect to those in inert atmosphere, even though the shapes of the curves seem to be quite similar. This different thermal behavior of the materials on the basis of the different atmosphere of nitrogen and air is substantially the same as that observed in a recent study concerning the multi-step thermal degradation of pure CGA [15]. The thermal behavior of all of the $\mathrm{SiO}_{2}$ /PEG/CGA materials under nitrogen atmosphere is shown in Figure 5a. The poor-CGA material $\left(\mathrm{SiO}_{2} / \mathrm{PEG}_{50} \mathrm{wt} \% / \mathrm{CGA}_{5 \mathrm{wt}} \%\right)$ shows a TG profile similar to that of $\mathrm{SiO}_{2} / \mathrm{PEG}_{50} \mathrm{wt} \%$ [46]: the water released is remarkably higher than those of the other $\mathrm{SiO}_{2} / \mathrm{PEG} / \mathrm{CGA}$ materials, but is comparable with that of $\mathrm{SiO}_{2} / \mathrm{PEG}_{50} \mathrm{wt} \%$, while the dehydration temperature is slightly shifted toward lower values (by a few degrees). The other $\mathrm{SiO}_{2} / \mathrm{PEG} / \mathrm{CGA}$ materials (with different amounts of CGA ranging from $10 \mathrm{wt} \%$ to $20 \mathrm{wt} \%$ ) showed a loss of water between $3 \%$ and $6 \%$. At higher temperatures, they undergo consecutive decomposition processes of CGA and PEG up to $600{ }^{\circ} \mathrm{C}$. In particular, the thermal decomposition of CGA seems to take place around $200{ }^{\circ} \mathrm{C}$ for $\mathrm{SiO}_{2} / \mathrm{PEG}_{50} \mathrm{wt} \% / \mathrm{CGA}_{5} \mathrm{wt} \%$, followed by that of PEG, which is sharper than that for $\mathrm{SiO}_{2} / \mathrm{PEG}_{50} \mathrm{wt} \%$ [46] (see Figure 5). It can be concluded that the presence of CGA does not significantly affect the thermal behavior of materials containing both $\mathrm{SiO}_{2}$ and PEG.

Unexpectedly, the oxidation environment seems not to influence the thermal behavior of these materials with respect to the inert one (air with respect to nitrogen, respectively), as it is clearly observed by comparing the TG curves in Figure 5b with those in Figure 5a (they are almost superimposable).

\subsection{Bioactivity Test}

The bioactivity of hybrid materials was evaluated by a Kokubo test [35]. FTIR analysis was used to detect the nucleation of hydroxyapatite on the surfaces of all of the samples after 21 days in SBF. Figure 6 shows the FTIR spectra of the hybrids, in which a new band at $630 \mathrm{~cm}^{-1}$ (inner plot in Figure 6) and the split of that at $570 \mathrm{~cm}^{-1}$ in two new ones at $575 \mathrm{~cm}^{-1}$ and $560 \mathrm{~cm}^{-1}$, respectively, were observed. These bands are due to the stretching of the $-\mathrm{OH}$ groups of hydroxyapatite and the vibrations of the $\mathrm{PO}_{4}^{3-}$ groups caused by the formation of the hydroxyapatite precipitate. Furthermore, the interaction of the hydroxyapatite layer with the $-\mathrm{OH}$ groups of the silica matrix was suggested by the displacement of $\mathrm{Si}-\mathrm{OH}$ band, from $955 \mathrm{~cm}^{-1}$ to $960 \mathrm{~cm}^{-1}$. The presence of $\mathrm{Si}-\mathrm{OH}$ groups on the surface of the hybrids stimulates hydroxyapatite nucleation, because these groups attract the $\mathrm{Ca}^{2+}$ ions present in the fluid, thus leading to an increase of the positive surface charge. The $\mathrm{Ca}^{2+}$ ions combine with the negative charge of the phosphate ions to form amorphous phosphate, which spontaneously transforms into hydroxyapatite $\left[\mathrm{Ca}_{10}\left(\mathrm{PO}_{4}\right)_{6}(\mathrm{OH})_{2}\right][47]$. 


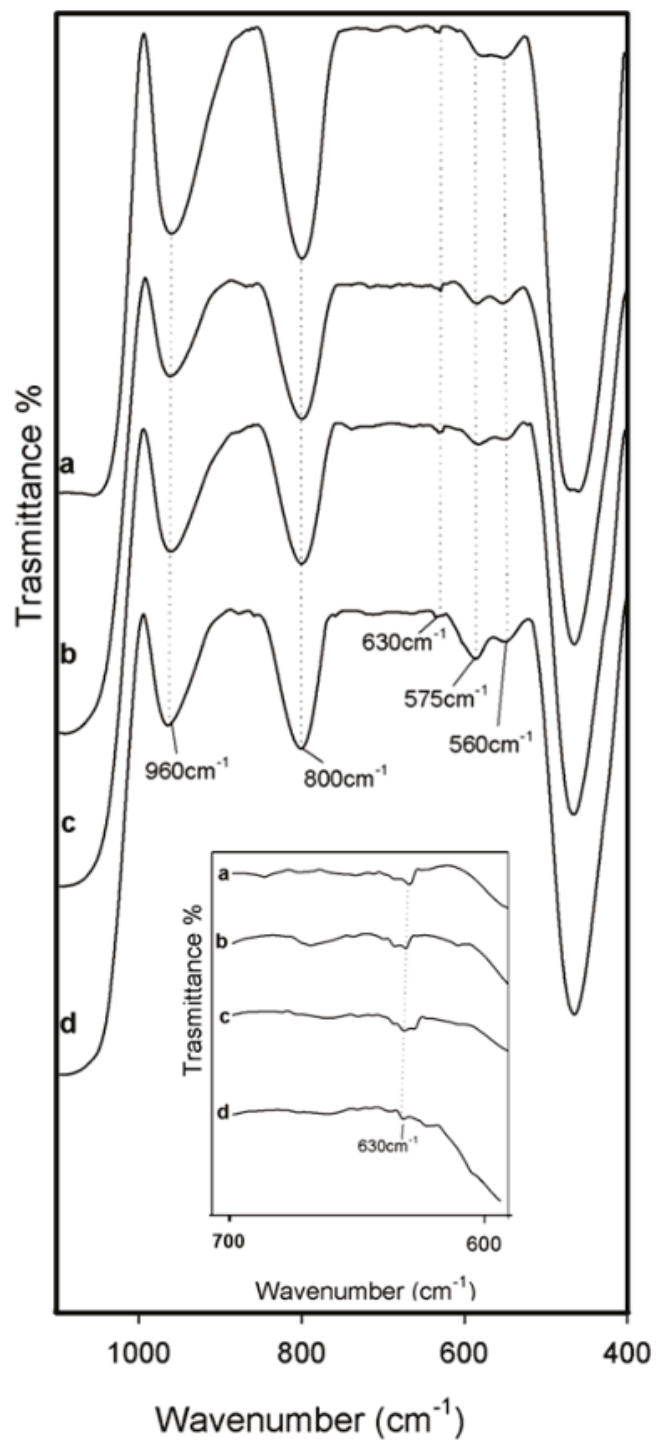

Figure 6. Representative FTIR spectra of (a) $\mathrm{SiO}_{2} / \mathrm{PEG}_{50 \mathrm{wt} \%} / \mathrm{CGA}_{5 \mathrm{wt} \%}$; (b) $\mathrm{SiO}_{2} / \mathrm{PEG}_{50} \mathrm{wt} \% / \mathrm{CGA}_{10} \mathrm{wt} \%$; (c) $\mathrm{SiO}_{2} / \mathrm{PEG}_{50} \mathrm{wt} \% / \mathrm{CGA}_{15} \mathrm{wt} \%$; (d) $\mathrm{SiO}_{2} / \mathrm{PEG}_{50} \mathrm{wt} \% / \mathrm{CGA}_{20} \mathrm{wt} \%$ after 21 days of exposure to SBF.

\section{Conclusions}

In this study, new hybrid materials with different percentages of CGA and PEG were prepared by sol-gel synthesis. FTIR analysis demonstrated that hydrogen bonds occurred in all of the materials between the inorganic silica matrix and the organic components. This result suggests that both the polymer and CGA are embedded in the matrix and interact with it.

The thermal behavior of the two different hybrid materials has been studied in both inert and oxidative atmosphere. The TG curves show that at higher temperatures, the materials undergo 
consecutive decomposition processes of CGA and polymer up to $600{ }^{\circ} \mathrm{C}$. The oxidation environment seems not to influence the thermal behavior of these materials with respect to the inert one.

Furthermore, the materials were soaked in a simulated body fluid to evaluate their bioactivity. The FTIR spectra showed also the formation of a hydroxyapatite precipitate on the sample surfaces, thus proving that the presence of both organic components does not affect the bioactivity of the silica matrix nor the thermal behavior.

Author Contributions: Formal analysis, E.T. and R.R.; Investigation, E.T. and R.R.; Methodology, S.V.C.; Project administration, M.C.; Supervision, M.C.; Writing—original draft, S.V.C.; Writing—review \& editing, M.C.

Funding: This research received no external funding.

Acknowledgments: The authors do not want to acknowledge any support.

Conflicts of Interest: The authors declare no conflict of interest.

\section{References}

1. Singh, D.; Singh, R.; Boparai, K.; Farina, I.; Feo, L.; Verma, A.K. In-vitro studies of ss 3161 biomedical implants prepared by fdm, vapor smoothing and investment casting. Compos. Part B Eng. 2018, 132, 107-114. [CrossRef]

2. Xu, L.-C.; Siedlecki, C.A. Effects of surface wettability and contact time on protein adhesion to biomaterial surfaces. Biomaterials 2007, 28, 3273-3283. [CrossRef] [PubMed]

3. Yang, S.Y.; Yu, H.; Gong, W.; Wu, B.; Mayton, L.; Costello, R.; Wooley, P.H. Murine model of prosthesis failure for the long-term study of aseptic loosening. J. Orthop. Res. 2007, 25, 603-611. [CrossRef] [PubMed]

4. Bridges, A.W.; García, A.J. Anti-inflammatory polymeric coatings for implantable biomaterials and devices. J. Diabetes Sci. Technol. 2008, 2, 984-994. [CrossRef] [PubMed]

5. Tsaryk, R.; Kalbacova, M.; Hempel, U.; Scharnweber, D.; Unger, R.E.; Dieter, P.; Kirkpatrick, C.J.; Peters, K. Response of human endothelial cells to oxidative stress on ti6al4v alloy. Biomaterials 2007, 28, 806-813. [CrossRef] [PubMed]

6. Danhof, M.; de Lange, E.C.; Della Pasqua, O.E.; Ploeger, B.A.; Voskuyl, R.A. Mechanism-based pharmacokinetic-pharmacodynamic (pk-pd) modeling in translational drug research. Trends Pharmacol. Sci. 2008, 29, 186-191. [CrossRef] [PubMed]

7. Zadeh, M.A.; Van Der Zwaag, S.; Garcia, S. Routes to extrinsic and intrinsic self-healing corrosion protective sol-gel coatings: A review. Self-Heal. Mater. 2013, 1, 1-18. [CrossRef]

8. Brinker, C.; Scherer, G. Sol-Gel Science: The Physics and Chemistry of Sol-Gel Processing; Academic Press: San Diego, CA, USA, 1989.

9. Barbe, C.; Bartlett, J.; Kong, L.; Finnie, K.; Lin, H.Q.; Larkin, M.; Calleja, S.; Bush, A.; Calleja, G. Silica particles: A novel drug-delivery system. Adv. Mater. 2004, 16, 1959-1966. [CrossRef]

10. Hakeem, A.; Zahid, F.; Zhan, G.; Yi, P.; Yang, H.; Gan, L.; Yang, X. Polyaspartic acid-anchored mesoporous silica nanoparticles for ph-responsive doxorubicin release. Int. J. Nanomed. 2018, 13, 1029. [CrossRef] [PubMed]

11. Banerjee, S.S.; Aher, N.; Patil, R.; Khandare, J. Poly(ethylene glycol)-prodrug conjugates: Concept, design, and applications. J. Drug Deliv. 2012, 2012. [CrossRef] [PubMed]

12. Moghimi, S.M.; Szebeni, J. Stealth liposomes and long circulating nanoparticles: Critical issues in pharmacokinetics, opsonization and protein-binding properties. Prog. Lipid Res. 2003, 42, 463-478. [CrossRef]

13. Rabanel, J.-M.; Hildgen, P.; Banquy, X. Assessment of peg on polymeric particles surface, a key step in drug carrier translation. J. Control. Release 2014, 185, 71-87. [CrossRef] [PubMed]

14. Vonarbourg, A.; Passirani, C.; Saulnier, P.; Benoit, J.-P. Parameters influencing the stealthiness of colloidal drug delivery systems. Biomaterials 2006, 27, 4356-4373. [CrossRef] [PubMed]

15. Owusu-Ware, S.K.; Chowdhry, B.Z.; Leharne, S.A.; Antonijević, M.D. Quantitative analysis of overlapping processes in the non-isothermal decomposition of chlorogenic acid by peak fitting. Thermochim. Acta 2013, 565, 27-33. [CrossRef]

16. Yutani, R.; Kikuchi, T.; Teraoka, R.; Kitagawa, S. Efficient delivery and distribution in skin of chlorogenic acid and resveratrol induced by microemulsion using sucrose laurate. Chem. Pharm. Bull. 2014, 62, 274-280. [CrossRef] [PubMed] 
17. Lou, Z.; Wang, H.; Zhu, S.; Ma, C.; Wang, Z. Antibacterial activity and mechanism of action of chlorogenic acid. J. Food Sci. 2011, 76. [CrossRef] [PubMed]

18. Granado-Serrano, A.B.; Angeles Martín, M.; Izquierdo-Pulido, M.; Goya, L.; Bravo, L.; Ramos, S. Molecular mechanisms of (-)-epicatechin and chlorogenic acid on the regulation of the apoptotic and survival/proliferation pathways in a human hepatoma cell line. J. Agric. Food Chem. 2007, 55, 2020-2027. [CrossRef] [PubMed]

19. Shin, H.S.; Satsu, H.; Bae, M.-J.; Zhao, Z.; Ogiwara, H.; Totsuka, M.; Shimizu, M. Anti-inflammatory effect of chlorogenic acid on the il-8 production in caco-2 cells and the dextran sulphate sodium-induced colitis symptoms in c57bl/6 mice. Food Chem. 2015, 168, 167-175. [CrossRef] [PubMed]

20. Bin, H.-S.; Jeong, J.-H.; Choi, U.-K. Chlorogenic acid promotes osteoblastogenesis in human adipose tissue-derived mesenchymal stem cells. Food Sci. Biotechnol. 2013, 22, 107-112. [CrossRef]

21. Shahidi, F.; Han, X.Q. Encapsulation of food ingredients. Crit. Rev. Food Sci. Nutr. 1993, 33, 501-547. [CrossRef] [PubMed]

22. Fang, Z.; Bhandari, B. Encapsulation of polyphenols-A review. Trends Food Sci. Technol. 2010, 21, 510-523. [CrossRef]

23. Li, Z.; Huang, D.; Tang, Z.; Deng, C.; Zhang, X. Fast determination of chlorogenic acid in tobacco residues using microwave-assisted extraction and capillary zone electrophoresis technique. Talanta 2010, 82, 1181-1185. [CrossRef] [PubMed]

24. Marques, V.; Farah, A. Chlorogenic acids and related compounds in medicinal plants and infusions. Food Chem. 2009, 113, 1370-1376. [CrossRef]

25. Perrone, D.; Farah, A.; Donangelo, C.M.; de Paulis, T.; Martin, P.R. Comprehensive analysis of major and minor chlorogenic acids and lactones in economically relevant brazilian coffee cultivars. Food Chem. 2008, 106, 859-867. [CrossRef]

26. Duarte, G.S.; Pereira, A.A.; Farah, A. Chlorogenic acids and other relevant compounds in brazilian coffees processed by semi-dry and wet post-harvesting methods. Food Chem. 2010, 118, 851-855. [CrossRef]

27. Kaur, N.; Lacasse, M.; Fürtös, A.; Waldron, K.C.; Morin, A. Sequential fractionation with concurrent chemical and toxicological characterization of the combustion products of chlorogenic acid. J. Chromatogr. A 2009, 1216, 4703-4712. [CrossRef] [PubMed]

28. Catauro, M.; Bollino, F.; Dell'Era, A.; Vecchio Ciprioti, S. Pure $\mathrm{Al}_{2} \mathrm{O}_{3} \cdot 2 \mathrm{SiO}_{2}$ synthesized via a sol-gel technique as a raw material to replace metakaolin: Chemical and structural characterization and thermal behavior. Ceram. Int. 2016, 42, 16303-16309. [CrossRef]

29. Catauro, M.; Dell'Era, A.; Vecchio Ciprioti, S. Synthesis, structural, spectroscopic and thermoanalytical study of sol-gel derived sio $_{2}-\mathrm{cao}-\mathrm{p}_{2} \mathrm{O}_{5}$ gel and ceramic materials. Thermochim. Acta 2016, 625, 20-27. [CrossRef]

30. Arrigo, R.; Teresi, R.; Gambarotti, C.; Parisi, F.; Lazzara, G.; Dintcheva, N.T. Sonication-induced modification of carbon nanotubes: Effect on the rheological and thermo-oxidative behaviour of polymer-based nanocomposites. Materials 2018, 11, 383. [CrossRef] [PubMed]

31. Nguyen, K.; Garcia, A.; Sani, M.A.; Diaz, D.; Dubey, V.; Clayton, D.; Dal Poggetto, G.; Cornelius, F.; Payne, R.J.; Separovic, F.; et al. Interaction of n-terminal peptide analogues of the $\mathrm{Na}^{+}, \mathrm{K}^{+}$-atpase with membranes. Biochim. Biophys. Acta Biomembr. 2018, 1860, 1282-1291. [CrossRef] [PubMed]

32. Blanco, I.; Bottino, F.A.; Cicala, G.; Latteri, A.; Recca, A. A kinetic study of the thermal and thermal oxidative degradations of new bridged poss/ps nanocomposites. Polym. Degrad. Stab. 2013, 98, 2564-2570. [CrossRef]

33. Blanco, I.; Bottino, F.A.; Cicala, G.; Latteri, A.; Recca, A. Synthesis and characterization of differently substituted phenyl hepta isobutyl-polyhedral oligomeric silsesquioxane/polystyrene nanocomposites. Polym. Compos. 2014, 35, 151-157. [CrossRef]

34. Catauro, M.; Pacifico, S. Synthesis of bioactive chlorogenic acid-silica hybrid materials via the sol-gel route and evaluation of their biocompatibility. Materials 2017, 10, 840. [CrossRef] [PubMed]

35. Kokubo, T.; Takadama, H. How useful is sbf in predicting in vivo bone bioactivity? Biomaterials 2006, 27, 2907-2915. [CrossRef] [PubMed]

36. Adeogun, M.; Fairclough, J.; Hay, J.; Ryan, A. Structure control in sol-gel silica synthesis using ionene polymers-Evidence from X-ray scattering. J. Sol-Gel Sci. Technol. 1998, 13, 27-30. [CrossRef]

37. Innocenzi, P. Infrared spectroscopy of sol-gel derived silica-based films: A spectra-microstructure overview. J. Non-Cryst. Solids 2003, 316, 309-319. [CrossRef] 
38. Simon, V.; Eniu, D.; Gritco, A.; Simon, S. Thermal and spectroscopic investigation of sol-gel derived aluminosilicate bioglass matrices. J. Optoelectron. Adv. Mater. 2007, 9, 3368-3371.

39. Nedelec, J.M.; Hench, L.L. Ab initio molecular orbital calculations on silica rings. J. Non-Cryst. Solids 1999, 255, 163-170. [CrossRef]

40. Yoshino, H.; Kamiya, K.; Nasu, H. Ir study on the structural evolution of sol-gel derived $\mathrm{SiO}_{2}$ gels in the early stage of conversion to glasses. J. Non-Cryst. Solids 1990, 126, 68-78. [CrossRef]

41. Silverstein, R.M.; Bassler, G.C.; Morrill, T.C. Spectroscopic Identification of Organic Compounds; John Wiley \& Sons: New York, NY, USA, 1981.

42. Catauro, M.; Bollino, F.; Nocera, P.; Piccolella, S.; Pacifico, S. Entrapping quercetin in silica/polyethylene glycol hybrid materials: Chemical characterization and biocompatibility. Mater. Sci. Eng. C 2016, 68, $205-212$. [CrossRef] [PubMed]

43. Catauro, M.; Bollino, F.; Papale, F.; Gallicchio, M.; Pacifico, S. Influence of the polymer amount on bioactivity and biocompatibility of $\mathrm{sio}_{2}$ /peg hybrid materials synthesized by sol-gel technique. Mater. Sci. Eng. C 2015, 48, 548-555. [CrossRef] [PubMed]

44. Catauro, M.; Renella, R.; Papale, F.; Vecchio Ciprioti, S. Investigation of bioactivity, biocompatibility and thermal behavior of sol-gel silica glass containing a high peg percentage. Mater. Sci. Eng. C 2016, 61, 51-55. [CrossRef] [PubMed]

45. Coates, J. Interpretation of infrared spectra, a practical approach. Encycl. Anal. Chem. 2000. [CrossRef]

46. Vecchio Ciprioti, S.; Catauro, M.; Bollino, F.; Tuffi, R. Thermal behavior and dehydration kinetic study of $\mathrm{SiO}_{2} /$ PEG hybrid gel glasses. Polym. Eng. Sci. 2017, 57, 606-612. [CrossRef]

47. Ohtsuki, C.; Kokubo, T.; Yamamuro, T. Mechanism of apatite formation on caosio ${ }_{2} \mathrm{p}_{2} \mathrm{O}_{5}$ glasses in a simulated body fluid. J. Non-Cryst. Solids 1992, 143, 84-92. [CrossRef]

(C) 2018 by the authors. Licensee MDPI, Basel, Switzerland. This article is an open access article distributed under the terms and conditions of the Creative Commons Attribution (CC BY) license (http:/ / creativecommons.org/licenses/by/4.0/). 
Article

\title{
Plateau-Shaped Flexible Polymer Microelectrode Array for Neural Recording
}

\author{
Jun-Min Kim ${ }^{1, *}$, Changkyun $\operatorname{Im}^{2}$ and Woo Ram Lee ${ }^{1}$ \\ 1 Medical Device Research Center Medical \& Health Device Division, Korea Testing Laboratory, \\ Seoul 52852, Korea; wool001@snu.ac.kr \\ 2 Dental Research Institute, Seoul National University School of Dentistry, Seoul 03080, Korea; \\ oingah@nate.com \\ * Correspondence: junmin83@gmail.com; Tel.: +82-10-9037-7297
}

Received: 6 November 2017; Accepted: 5 December 2017; Published: 8 December 2017

\begin{abstract}
Conventional polymer multielectrode arrays (MEAs) have limitations resulting from a high Young's modulus, including low conformability and gaps between the electrodes and neurons. These gaps are not a problem in soft tissues such as the brain, due to the repopulation phenomenon. However, gaps can result in signal degradation when recording from a fiber bundle, such as the spinal cord. Methods: We propose a method for fabricating flexible polydimethylsiloxane (PDMS)-based MEAs featuring plateau-shaped microelectrodes. The proposed fabrication technique enables the electrodes on the surface of MEAs to make a tight connection to the neurons, because the wire of the MEA is fabricated to be plateau-shaped, as the Young's modulus of PDMS is similar to soft tissues and PDMS follows the curvature of the neural tissue due to its high conformability compared to the other polymers. Injury caused by the movement of the MEAs can therefore be minimized. Each electrode has a diameter of $130 \mu \mathrm{m}$ and the 8-channel array has a center-to-center electrode spacing of $300 \mu \mathrm{m}$. The signal-to-noise ratio of the plateau-shaped electrodes was larger than that of recessed electrodes because there was no space between the electrode and neural cell. Reliable neural recordings were possible by adjusting the position of the electrode during the experiment without trapping air under the electrodes. Simultaneous multi-channel neural recordings were successfully achieved from the spinal cord of rodents. We describe the fabrication technique, electrode 3D profile, electrode impedance, and MEA performance in in vivo experiments in rodents.
\end{abstract}

Keywords: fabrication; multielectrode array (MEA); PDMS; PDMS etching; plateau-shaped electrode; recessed electrode; spinal cord signal recording; underexposure

\section{Introduction}

A common goal of physiologists and bioengineers is to interface with and elucidate the properties of excitable cells within intact tissues [1]. For example, a brain-computer interface (BCI) couples the nervous system to a device that can either stimulate the tissue or record neural activity, or perform both in a closed-loop system [2]. A BCI typically includes a computer for analyzing signals and an electrode for stimulating or recording a neural signal [2,3]. The electrode is a transducer that converts the activity of an ionic current into an electrical potential, so it directly affects the quality and reliability of the BCI.

Electrodes can be divided into two types: Non-invasive and invasive. Although non-invasive techniques have the advantage of being safe, the spatial resolution is generally lower than that of invasive methods $[4,5]$. The signal acquired from a non-invasive electrode includes the effect of the activity signal of neuroglia surrounding the stimulated neurons, rather than directly the activity of the neurons being stimulated. However, invasive electrode technologies have higher spatial resolution, and with the rapid growth of computing power, can process a large amount of information simultaneously 
through a multielectrode array (MEA) [6,7]. MEAs can be classified as either probe-type or planar-type. The probe type is commonly made of rigid materials, such as glass, silicon, and metal, in order to penetrate the tissue surface $[7,8]$. Inevitably, during the insertion process, a wound occurs and, after insertion, damage to the electrode surrounding the tissue occurs due to the friction caused by the micro-movement of the electrode. Due to the immune rejection and inflammatory response, long-term experiments by using a probe-type electrode are difficult $[9,10]$.

To overcome this drawback, rigid planar MEAs have been developed to interface with the surface of tissues [11]. Glass or silicon wafers are mainly used as substrate materials; particularly, glass wafers are widely used with transmission microscopy. If the signals from the deep part of the neural tissue are to be recorded or the deep part is to be stimulated, a probe-type MEA is required. If the region of interest is close to the surface of the tissue, a rigid planar MEA can be a good alternative in terms of biocompatibility. However, a rigid planar MEA cannot closely follow the curvature of the tissue, which is a disadvantage.

In order to improve the ability of MEA to interface closely and effectively with the tissue surface, MEAs have been fabricated on flexible substrates such as polyimide, parylene, and silk [12-15]. The rigid planar MEA is commonly used in vitro, while the flexible planar MEA is used widely in vivo because it can cover the complex surface of tissue well. However, as parylene and polyimide are more rigid than neural tissues, micro-movement of flexible planar MEAs can cause tissue injury. In the case of silk electrodes, the electrode location cannot be adjusted after the initial positioning [15].

The PDMS polymer has been used to solve these problems [16-20]. As the Young's modulus of PDMS is similar to that of neural tissues (approximately 1.0 MPa) $[13,17,20]$, tissue damage is reduced compared to other polymers. In addition, the location of PDMS-based MEAs can be adjusted easily during the experiment, due to good conformability $[17,19,21,22]$. In spite of these merits, PDMS has not been widely used as a substrate material because it is difficult to apply existing semiconductor processing techniques. The PDMS surface has low surface energy, so it is difficult to deposit metal on it. As it is chemically stable, wet/dry etching is difficult [22,23].

So far, the all-flexible planar MEAs that have been developed have a similar structure, regardless of the type of substrate. First, metal wires are fabricated on a flexible substrate, and after encapsulating the sample using an insulator, electrodes are manufactured by etching the insulator above the wire $[12,16,21]$. These manufacturing processes always generate a recessed structure over the electrode, which is surrounded by the insulator. As this recessed structure increases the distance between the electrode and the neuron, recording and stimulating performances are degraded. Air trapping phenomena can also occur in this area, disturbing the electrode-neuron interface. In order to prevent air trapping, oxygen plasma treatment has been commonly used [18], but with time, the surface characteristics of PDMS change from hydrophilic to hydrophobic. Therefore, this method is not a permanent solution, and new technology is needed.

In this paper, we propose a new fabrication process to manufacture a PDMS-based planar MEA that does not generate a recessed structure, and instead produces a plateau-shaped electrode. Moreover, we measure the physical characteristic and impedance of the developed MEA, and evaluate air trapping. Finally, we evaluate the performance of the proposed MEA by recording neural activity in vivo in the spinal cord of an anesthetized rodent, stimulated by touching the limb and pelvic regions.

\section{Methods}

\subsection{Electrode Structure and Fabrication}

Figure 1a shows a 3D structural view of the PDMS-based plateau MEA. A plateau MEA has a total of 8 electrodes and requires an additional interface for connecting MEA and recording equipment. The method of connecting electrodes to external equipment using epoxy glue and soldering was common, but gold thin films on PDMS can be easily broken by external force. The zero-insertion force (ZIF) connector eliminated the need to use epoxy glue or soldering. The ZIF connector (FH12-16S-0.5SH, 
HIROSE, Tokyo, Japan) was used a device of 16-pin with $0.5 \mathrm{~mm}$ pitch. The developed MEA has 8 pads, and we designed the pad size that one pad can use two ZIF pins. We designed the interface pad with a width of $700 \mu \mathrm{m}$ and a pitch of $1 \mathrm{~mm}$. The electrodes, wires, and interface pads of the zero-insertion force (ZIF) connector are made of gold, and the base material of the electrode is made of PDMS. Figure 1b shows the electrode cross-section. The thickness of the MEA is approximately $150 \mu \mathrm{m}$, and thickness can be controlled easily through the PDMS spin-coating speed. The thickness of the substrate inserted into the ZIF connector should be at least $300 \mu \mathrm{m}$. Thus, a plastic plate having a thickness of $200 \mu \mathrm{m}$ was placed behind the MEA and used. The electrode, pads, and wires are parts of a single body, and only the electrode connecting to the neurons and the pad computer interface are exposed to air, as shown in Figure $1 \mathrm{~b}$. The plateau electrode structure not only enables the electrode to make solid contact with the neurons, but also improves electrode performance by preventing air from becoming trapped between the electrode and tissue. Based on these characteristics, after attaching the MEA on a brain or spinal cord, we could adjust the location of MEA to optimize the biological signal recorded.

(a)

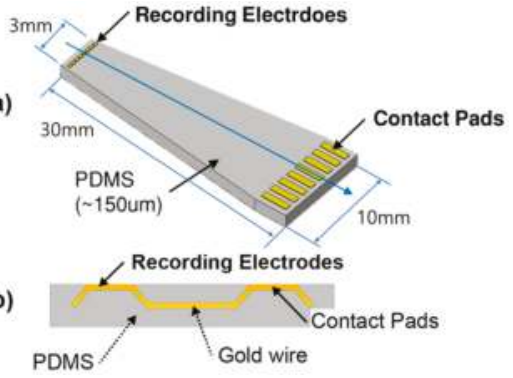

Figure 1. 3D schematic of the electrode array. (a) Schematic of a polydimethylsiloxane (PDMS)-based multielectrode array. (b) Cross-section along the blue arrowed line in (a).

The sequential processes for fabricating the MEA are shown schematically in Figure 2, and described in detail below. First, a cleaned silicon wafer was coated with parylene (Parylene-C, CNC Chemical, San Clemente, CA, USA) to obtain weak bonding between the silicon wafer and PDMS film. This prevented the metal from joining with the silicon wafer, and helped to strip the MEA from the wafer in the final process. A parylene layer of $3 \mu \mathrm{m}$ was deposited using parylene deposition equipment (PDS2010, SCS Inc., Glendale, CA, USA), as shown in Figure 2a. A sacrificial post was subsequently built on the sample via photolithography, as shown in Figure $2 \mathrm{~b}$. The negative photoresist (DNRL300-40, DONGJIN SEMICAM Co., Seoul, Korea) used in this research was spin-coated at $1300 \mathrm{rpm}$ for $20 \mathrm{~s}$, and a thickness of $7 \mu \mathrm{m}$ was achieved. Next, a double-coating scheme was used to achieve a photoresist layer thickness of $20 \mu \mathrm{m}$. The sample obtained after the second coating was exposed at $25 \mathrm{~mW} / \mathrm{cm}^{2}$ for $18 \mathrm{~s}$ using exposure equipment (MA6 Aligner, SUSS MicroTec Group, Corona, CA, USA). Subsequently, in order to adjust the inclination of the sacrificial post, we placed a $160-\mu \mathrm{m}$ transparent polyethylene terephthalate (PET) film between the sample and mask. The inclination of the post could be controlled by regulating the thickness of the PET film and the exposure time. The sample was subsequently treated with oxygen plasma by using reactive ion etching (RIE) (RIE 80 Plus, Oxford Instruments, Abingdon, UK) in order to increase the adhesion force between parylene and PDMS. PDMS (Sylgard R184, Dow Corning, Co., Midland, MI, USA) was spin-coated onto the sample at $1500 \mathrm{rpm}$ for $60 \mathrm{~s}$, as shown in Figure 2c. The sample was subsequently cured in an $80{ }^{\circ} \mathrm{C}$ oven for $2 \mathrm{~h}$. After curing, the PDMS layer was wet-etched using a mixture of tetrabutylammonium fluoride (TBAF, Tokyo Chemical Industry, Tokyo, Japan) and n-methylpyrrolidone (NMP, Tokyo Chemical Industry, Tokyo, Japan) until the sacrificial post was revealed. The PDMS etchant consisted of TBAF and NMP mixed at a ratio of 1:10 $(v / v)$ [24]. After PDMS etching, the photoresist residue 
on the electrodes and pads was cleaned using NMP. The sample was subsequently cleaned using de-ionized (DI) water, and sonication was performed for $5 \mathrm{~min}$ in DI water. Before depositing gold onto the PDMS in the hole regions, 2-hydroxyethyl methacrylate (HEMA) was polymerized on the PDMS and parylene film by using oxygen plasma to increase metal adhesion. The polymerization method consisted of three steps: The first step consisted of increasing the surface energy of the polymer via oxygen plasma, which helps HEMA combine with the PDMS and parylene [25]. The parameters of the RIE equipment for the oxygen plasma treatment were as follows: a pressure of 75 mTorr, oxygen flow rate of $20 \mathrm{sccm}$, and power of $100 \mathrm{~W}$; these parameters were the same in all steps.
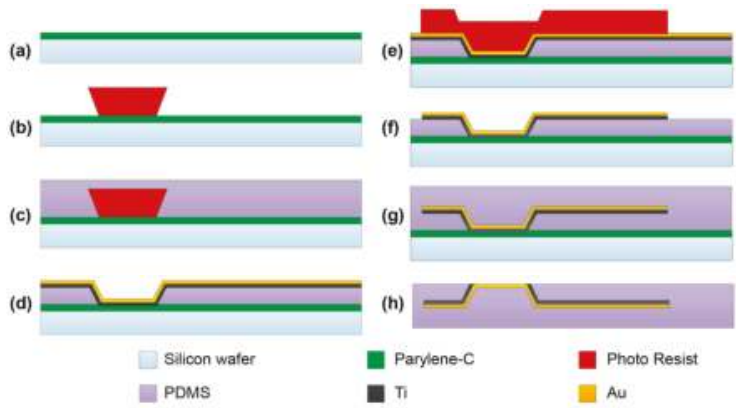

Figure 2. Schematic of fabrication process for a PDMS-based plateau electrode array.

In the second step, HEMA was spin-coated onto the sample at $1500 \mathrm{rpm}$ for $30 \mathrm{~s}$. The oxygen plasma treatment was repeated in the third step. Thus, we could graft an amphiphilic HEMA film onto the PDMS surface. After surface treatment, titanium with a thickness of $300 \AA$ was deposited on the sample using an electron gun evaporator (ZZS550, MAESTECH, Pyeongtaek, Korea) to increase the adhesion between the gold and PDMS. Subsequently, a gold layer with a thickness of $3000 \AA$ was deposited. Although gold was used as the electrode material in this study, according to recent trends in the manufacture of electrodes, $\mathrm{Pt}, \mathrm{IrO} 2$, or TiN can also be used. Titanium was deposited at $3 \AA / \mathrm{s}$ until a thickness of $300 \AA$ was obtained, and gold was deposited at $2 \AA$ /s until a thickness of $3000 \AA$ was obtained, as shown in Figure $2 \mathrm{~d}$.

In order to pattern the gold layer, photoresist was coated under the same conditions as described earlier, and exposed for $20 \mathrm{~s}$ without the PET film, as shown in Figure 2e. The gold layer was etched by a solution of iodine and potassium iodide at room temperature. After titanium was revealed, it was etched using a buffered oxide etcher (BOE), as shown in Figure 2f. PDMS was spin-coated for strong bonding after treating the PDMS surface by oxygen plasma. The encapsulating PDMS layer was manufactured by spin-coating at $700 \mathrm{rpm}$ for $60 \mathrm{~s}$, as shown in Figure $2 \mathrm{~g}$. The thickness of the PDMS encapsulation layer was approximately $100 \mu \mathrm{m}$, depending on the spin-coating speed. The outline of the MEA was cut by a Nd:YVO 4 laser (Series 3500 UV Laser System, DPSS Lasers, Inc., Santa Clara, CA, USA), and the process conditions were as follows: power of $2.4 \mathrm{~W}$, speed of $2 \mathrm{~mm} / \mathrm{s}$, wavelength of $10 \mu \mathrm{m}$, and frequency of $20 \mathrm{kHz}$. The sample was soaked in $70 \%$ ethanol solution for $30 \mathrm{~min}$ to weaken adhesion between the parylene and silicon wafer. The parylene layer above the gold electrode was easily etched using the RIE system for $10 \mathrm{~min}$ under an oxygen flow rate of $20 \mathrm{sccm}$, pressure of 350 mTorr, and power of $65 \mathrm{~W}$, as shown in Figure 2h. After removing the parylene, the Ti layer was etched using BOE, and the sample placed in DI water for 1 day to remove contaminants. 


\subsection{Device Characterization}

\subsubsection{Electrode Profile}

The most important aspect of the electrode manufacturing was ensuring that the holes in the PDMS film had a gentle slope. The reasons to fabricate holes with a gentle slope can be explained as follows: As the electrode of the RIE system is commonly oriented perpendicular to the wafer, the plasma has a vertical direction. If the wall of the PDMS hole is vertical to the wafer, plasma processing does not perform as well. There are many ways to deposit the metal, and categorized in order of step-coverage criteria, sputtering, electron-gun, and thermal evaporation methods all exhibited excellent coverage characteristics. If we were to deposit metal on a vertical wall using a thermal evaporator, the metal would not uniformly cover the wall, but rather accumulate in the corners. In order to solve these problems, holes with a gentle slope are necessary. Such holes facilitate the surface treatment of PDMS, and enable various metal depositions on the sample.

A negative photoresist film of thickness $20 \mu \mathrm{m}$ was manufactured to produce a variety of inclined PDMS holes. While adjusting the contact conditions of the photomask and exposure energy, we measured the slope angle of the sacrificial post. The photomask contact conditions were approximate, soft, and hard, and the exposure time was tested at intervals of 2 s over 12-20 s. We manufactured sacrificial posts using the photolithographic conditions obtained from the above experiment, and coated the PDMS film such that it was thicker than the height of the post. Bare, fluorocarbon (FC), and HEMA-coated wafer were prepared to obtain a PDMS film of proper thickness. Uncured PDMS was diluted in tert-butyl alcohol (TBA), and 50\% and 25\% solutions (w/w) were prepared in order to determine which characteristics depended on concentration [26]. We spin-coated PDMS on the prepared wafer for $60 \mathrm{~s}$ at 500,1000, 2000, and $4000 \mathrm{rpm}$, and measured the film thickness using Alpha-Step (Nanospec AFT/200, KLA-TENCOR, Milpitas, CA, USA). The alpha-step equipment was developed for measuring level differences on the sample surface. When the stylus is scanned the sample surface, it senses the pressure caused by a level difference, and measures the height of the level. The stylus was a diamond needle, which had a diameter of $10 \mu \mathrm{m}$, stylus force of $17.3 \mathrm{mg}$, scan speed of $20 \mu \mathrm{m} / \mathrm{s}$, sampling rate of $50 \mathrm{~Hz}$, and vertical resolution of $25 \AA$.

TBAF solutions of four types $(6,9,16,25 \%(v / v))$ were prepared for measuring the etching rate of PDMS. After an aluminum tape was attached to the PDMS sample, it was soaked in TBAF solution for $20 \mathrm{~min}$. Subsequently, the tape was removed from the PDMS sample, and the etching rate of PDMS was evaluated using alpha-step.

The 3D profiler $\mu$ Surf (NanoFocus, Oberhausen, Germany) was used to analyze the 3D structure of the electrode. The 3D profiler could measure the 3D profile without contact using confocal multi-pinhole (CMP) technology; however, due to the measurement mechanism using reflected visible light, we could not measure transparent materials such as glass and thin PDMS. In order to verify the 3D hole profile of the PDMS film, we measured the electrode region after gold deposition.

\subsubsection{Surface Treatment}

PDMS exhibits poor adhesion to metal, and the hydrophobic surface had to be modified to a hydrophilic surface by using an oxygen plasma treatment. However, this method is not permanent because the hydrophilic PDMS surface gradually turns hydrophobic over time. If the metal deposition and plasma treatments occur more than $24 \mathrm{~h}$ apart, it would be difficult to use an oxygen plasma process. Instead, in this study, we used a three-step surface modification process to treat the PDMS surface. The first step included modifying the PDMS surface using an RIE system, which enables the HEMA to spread out stably. The second step consisted of applying the HEMA coating, and the third step consisted of grafting the HEMA polymer using oxygen plasma. Each sample was stored $1 \mathrm{~h}$ and $48 \mathrm{~h}$ at $25^{\circ} \mathrm{C}$ before gold was subsequently deposited. Furthermore, HEMA-treated samples were maintained each 1 day and 7 days at $25^{\circ} \mathrm{C}$ before gold was deposited. The Scotch tape test was used to test the adhesion of the gold film to PDMS [27]. The experiments were conducted with two types 
of tapes that have different adhesive forces-one having an adhesion force of $2.5 \mathrm{~N} / 100 \mathrm{~mm}(810$, $3 \mathrm{M}$ Inc., Maplewood, MN, USA) was denoted as tape 1, and the other having an adhesion force of 14 N/100 mm (483, 3M Inc., Maplewood) was denoted as tape 2. Pieces of adhesive tape were placed on the gold film and a pressure of $64.5 \mathrm{gf} / \mathrm{cm}^{2}$ was applied for $5 \mathrm{~min}$. To apply uniform pressure on the adhesive tapes, a slide glass of weight $500 \mathrm{~g}$ was placed on the tape. When the adhesive tapes were released from the PDMS, a segment of the gold film could be detached from the PDMS along with the tape, depending on the adhesive force between the gold film and PDMS.

\subsubsection{Electrode Impedance Measurement}

The electrical properties of the electrodes were evaluated using impedance spectroscopy. The recessed electrode structure was placed $12 \mu \mathrm{m}$ below the surface of the MEA, and the other electrode was mounted superficially on the surface of the MEA. In the case of the recessed electrode, due to the hydrophobic surface of PDMS, oxygen plasma modification should be performed to prevent air trapping. We used the method and setup reported in Ref. [28] to measure the electrode impedance. One MEA that we used has eight electrodes of the same size, and ten MEAs have a total of 80 electrodes. By measuring the impedance five times from 80 electrodes, a total of 400 values can be obtained. The impedance result was obtained by averaging these values. The impedance values of recording sites (each site having dimensions of $130 \mu \mathrm{m} \times 130 \mu \mathrm{m}$ ) were determined in a phosphate-buffered saline solution (PBS solution, Gibco \#10010, Invitrogen Life Technologies, Waltham, MA, USA) at room temperature using an impedance analyzer (IM6e, Zahner-Elektrik GmbH \& Co., Kronach, Germany). A three-electrode arrangement was used, with an $\mathrm{Ag} / \mathrm{AgCl}$ reference electrode (K0260, ATFrontier, Anyang, Korea) and Pt counter electrode (RDE0021, ATFrontier, Anyang, Korea). A $5 \mathrm{mV}$ RMS sine wave was applied to the electrodes with the frequency ranging logarithmically from $100 \mathrm{~Hz}$ to $100 \mathrm{kHz}$.

\subsubsection{Surface Contact Performance}

A surface contact experiment was performed to examine whether the two types of electrode were properly attached to the nervous system in solution [18]. After the MEAs were immersed in DI water in a petri dish, a cover glass $(24 \mathrm{~mm} \times 40 \mathrm{~mm} \times 0.2 \mathrm{~mm})$ was attached to the electrode surface, and subsequently removed. After attaching the two types of MEA to the cover glass in the same manner, the electrode was inspected using an optical microscope.

\subsection{In Vivo Experiments}

Female rats (Sprague-Dawley, Taconic, Köln, Germany) were used for the experiment (Protocol number: Hallym2016-58). The animals were wild-type and had no history of surgical treatment or disability. Furthermore, they were bred in isolation from external environment by automated equipment, and water and food were provided every $12 \mathrm{~h}$. They were anesthetized using an intramuscular injection with Zoletil (Virbac SA, Carros, France) $0.2 \mathrm{~mL}$ and Rompun (Bayer Pharmaceuticals, Berlin, Germany) $0.1 \mathrm{~mL}$. After inserting the catheter into the urinary tract of the anesthetized rats and emptying the bladder, a laminectomy was performed from L2 to L1 of the spinal cord. After disinfecting from the 13th thoracic vertebrae to the 3rd lumbar, the spinal cord was exposed by removing the bump via laminectomy. The dura mater of spinal cord was removed, and the spinal cord was fixed using stereotaxic equipment (Model 1040, David Kopf Instruments, Tujunga, CA, USA). First, the neural signal was recorded by an 8-channel tungsten wire electrode. The sampling rate of the recorder (Micro1401-3, CED, Salford, UK) was set to $25 \mathrm{kHz}$, the gain was $1 \mathrm{k}$, and frequency range of the band-pass filter was 0.3 to $1000 \mathrm{~Hz}$. We searched for places where the neural signal was generated by pressure stimulation using a tungsten electrode. After selecting a location where the firing rate was high, the neural signal was recorded. Subsequently, using the proposed MEA with 8-channel, we recorded the signal in the same location under the same conditions. The pad area of the proposed MEA is connected to the recorder equipment using the ZIF connector. The 
recorder consists of amplifier and data acquisition device. The recorder connected to the computer and records the signal in real time using USB interface. A constant-pressure stimulus was applied to the pelvic and limb regions using a custom-built touch-test sensory evaluator. A stimulation sequence was composed of $30 \mathrm{~s}$ of rest, $30 \mathrm{~s}$ of stimulus, and $30 \mathrm{~s}$ of rest, and the experiment was repeated 2-3 times. We recorded both single-unit signals and the local field potential using the PDMS electrode.

\section{Results and Disscussion}

The overall appearance and the electrode region of the fabricated PDMS-based plateau MEA are shown in Figure 3. The dimensions of the gold electrodes were $130 \mu \mathrm{m} \times 130 \mu \mathrm{m}$, and the electrodes were mounted superficially on the PDMS surface. The experiments characterized the electrode 3D profile, electrode impedance, contact performance, and in vivo performance. The following sections describe these experimental results.
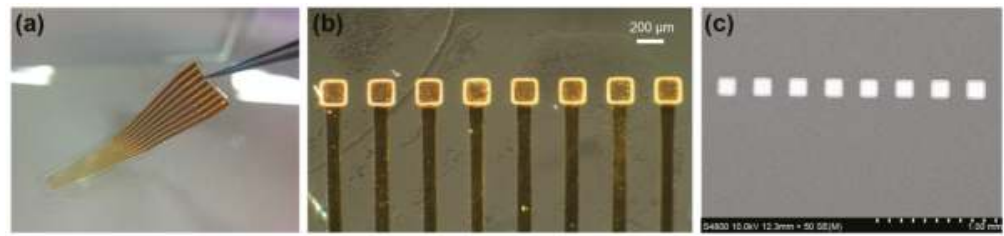

Figure 3. Images of fabricated PDMS-based plateau MEA. (a) The overall appearance of the MEA. The sacrificial photoresist post of this sample was spin-coated twice at $1300 \mathrm{rpm}$ for $20 \mathrm{~s}$. The thickness was $20 \mu \mathrm{m}$ and the depth of the PDMS hole was $11 \mu \mathrm{m}$. (b) Optical microscope image of the plateau microelectrodes. The bright region around the electrode is the gold film on the wall of the PDMS hole. (c) SEM image of the plateau microelectrodes. As the gold wires are inside the PDMS, we could not observe them using SEM. The electrodes were deposited with dimensions of $100 \mu \mathrm{m} \times 100 \mu \mathrm{m}$, but the SEM measurements show dimensions of $130 \mu \mathrm{m} \times 130 \mu \mathrm{m}$. The increase in electrode size is caused by the addition of the $160 \mu \mathrm{m}$ PET film and the shrinking and etching of PDMS as a result of the NMP and TBAF solutions.

\subsection{Electrode Profile}

The proposed plateau MEA features microelectrodes with a plateau structure. While manufacturing these electrodes, the most important aspect was the manufacture of the sacrificial post, which has an inclined wall, as the shape of the cured PDMS was determined by the shape of the sacrificial posts. Furthermore, both the spin-coating speed of the uncured PDMS and the etching time of the cured PDMS affected the 3D profile of the electrode. While manufacturing the sacrificial posts, the slope of the wall depended on two factors - the gap between wafer and photomask, and the exposure energy. Based on the gap between wafer and photomask, photolithography conditions were classified as approximate, soft-contact, hard-contact, or vacuum-contact. The larger the gap between sample and photomask, the larger the effect of optical diffraction; therefore, the gap was a factor that controlled the inclination angle of the post wall. Sufficient time was required when the UV light reached the lower part of the photoresist film, and we could control the amount of UV light absorption in the upper and lower sides by adjusting the exposure time. In the case of a negative photoresist, if the exposure time is reduced, the upper part of the photoresist film absorbs more energy than the lower part. Consequently, the lower region of photoresist is more developed than the upper region. Figure 4 shows scanning electron microscope (SEM) images of the inclined sacrificial post according to the exposure conditions. We demonstrated that the sidewall slope of the sacrificial posts could be controlled by applying the PET film and adjusting the UV light exposure time, as shown in Figure 4. The photomask employed in this study had a circular pattern of diameter $50 \mu \mathrm{m}$. By comparing Figure $4 a$,b, or Figure $4 c, d$, it can be observed that the longer the exposure time, the larger the slope 
angle. Comparing Figure $4 a, b$, we could observe that the PET film results in a decrease of slope angle. Through the above results, it can be observed that a larger gap between wafer and photomask or a shorter exposure time can both reduce the slope angle.
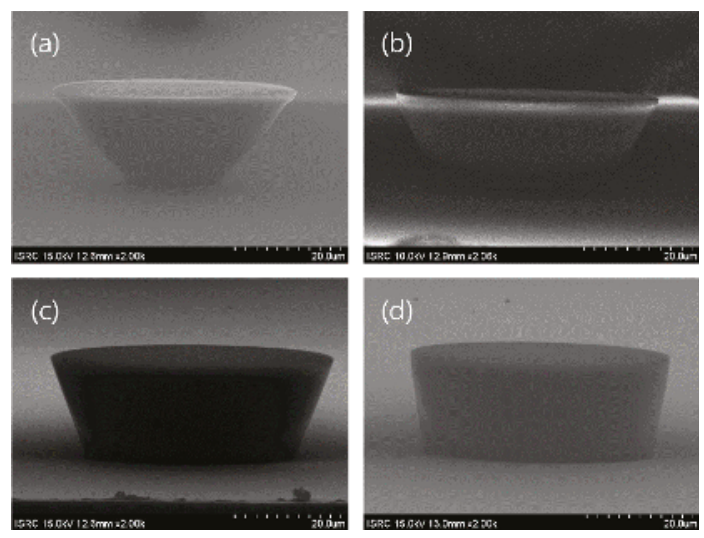

Figure 4. SEM images of the inclined sacrificial post under different exposure conditions. The height of the photoresist post was $20 \mu \mathrm{m}$, and the pattern of the photomask was a circle with a diameter of $50 \mu \mathrm{m}$. (a) A $160 \mu \mathrm{m}$ PET film was placed between the wafer and photomask, and the exposure time was $14 \mathrm{~s}$ at $25 \mathrm{~mW} / \mathrm{cm}^{2}$. The angle of the resulting post was $50^{\circ}$, obtained by averaging 9 individual posts from five rounds of fabrication. (b) The same PET film was used, with an exposure time of $14 \mathrm{~s}$. The measured angle was $63^{\circ}$. (c) Soft contact was used, the exposure time was $14 \mathrm{~s}$, and the angle was $72^{\circ}$. (d) Hard contact was used, the exposure time was $14 \mathrm{~s}$, and the angle was $83^{\circ}$.

We measured how the thickness of the PDMS film depended on the spin-coating speed, viscosity, and type of substrate. The film thickness of cured PDMS depended on the spin-coating speed and viscosity, as shown in Figure 5a. In previous studies, the PDMS film was manufactured to be as thin as possible by spin-coating on posts with a height of $20 \mu \mathrm{m}$ [21]. This method had a low yield, because PDMS residue was formed on the sacrificial posts, as shown in Figure 5b. We attempted to remove the PDMS residue on the posts by using a wet-etching process; however, the non-uniform thin PDMS film was torn frequently, as shown in Figure 5d. This problem could be solved by applying uncured PDMS thicker than the photoresist posts, as shown in Figure $5 c$. Based on the results of Figure $5 \mathrm{a}$, uncured PDMS was spin-coated on the inclined post with a thickness of $40 \mu \mathrm{m}$ to obtain good uniformity over $20-\mu \mathrm{m}$ posts. After etching the cured PDMS and removing the photoresist posts using NMP, a PDMS film with holes could be obtained; however, if the uniformity of the PDMS film was poor, the setup of the etching process was difficult, as shown in Figure 5b,d. The PDMS film with holes could be obtained using a uniform PDMS film, as shown in Figure 5e. Methods for etching the PDMS include dry and wet methods. As dry-etching was very slow $(0.1 \mu \mathrm{m} / \mathrm{min})$ [24], we used the wet-etching method because tens of micrometers of PDMS had to be removed. The wet-etching solution was a mixture of TBAF and NMP, and the PDMS etching rates at room temperature are shown in Table 1. The $25 \%$ TBAF solution exhibited a high etching rate, but PDMS slurry leftover after etching was a drawback [23]. While the $6 \%$ solution exhibited a low etching rate, the etching time and exposure time to NMP were prolonged, which could adversely affect the PDMS film [29].

Table 1. PDMS wet-etching rates using TBAF solution.

\begin{tabular}{ccccc}
\hline NMP:TBAF $(v / v)$ & $3: 1(25 \%)$ & $5: 1(12 \%)$ & $10: 1(9 \%)$ & $15: 1(6 \%)$ \\
\hline Etching rate $(\mu \mathrm{m} / \mathrm{min})$ & 3.7 & 2.8 & 2 & 1.3 \\
\hline
\end{tabular}


(a)
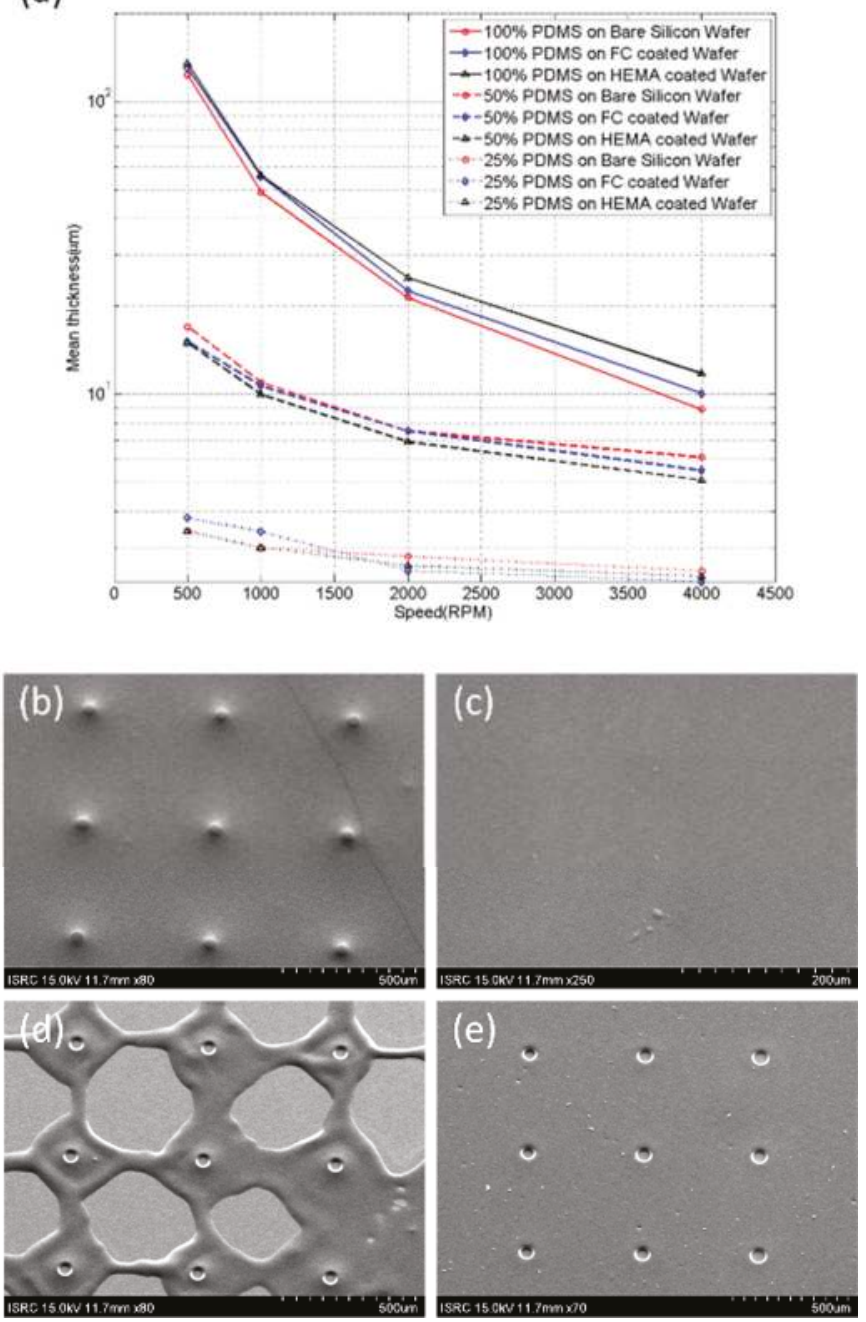

Figure 5. Characterization of PDMS spin-coating. (a) The thickness of PDMS film depended on spin-coating speed. The spin-coating speeds were 500, 1000, 2000, and $4000 \mathrm{rpm}$ for $60 \mathrm{~s}$. Each point on the plot corresponds to measurements on 25 samples from 5 rounds of fabrication. (b-e) SEM images measuring the uniformity of the PDMS film. (b) Uncured PDMS spin-coated with a thickness of $10 \mu \mathrm{m}$ on a photoresist post with a height of $20 \mu \mathrm{m}$. (c) Uncured PDMS spin-coated with a thickness of 40 $\mu \mathrm{m}$ on a photoresist post with a height of $20 \mu \mathrm{m}$. The photoresist posts are not revealed on the surface due to the thick PDMS film. (d) Cured PDMS of (b) etched by 9\% TBAF solution for $4 \mathrm{~min}$. The PDMS in the nearby and far posts were thick and thin, respectively. Hence, we could not obtain a uniform PDMS film. (e) Cured PDMS of (b) etched by $9 \%$ TBAF solution for $10 \mathrm{~min}$. Uniform PDMS film with holes could be obtained.

The PDMS etching method has been developed in various ways $[17,18,21,23,24]$. A method that uses sacrificial posts should be employed in order to adjust the inclination of the PDMS hole [18]. Therefore, we manufactured sacrificial posts with various inclinations using underexposure and 
approximate exposure techniques. After obtaining sacrificial posts, the standard method to obtain a thin PDMS film with holes would be to spin-coat PDMS onto the sample at very high speed. However, if this method was used, the PDMS film would be thick near the sacrificial posts and thin far from the posts; thus, the uniformity of film would be degraded. Additionally, PDMS residues on the sacrificial pillar caused problems [23]. To solve these two problems simultaneously, after coating the PDMS to be thicker than the sacrificial posts, the overall PDMS film was wet-etched until the posts were revealed.

Figure 6 shows the 3D profile measurements of the electrode. In order to determine and measure the key fabrication parameters of the plateau electrode structure, an analysis of the electrode profile was necessary. The 3D profiler could not detect a transparent structure due to the use of white visible light; thus, the electrode profile was measured after gold deposition. Figure 6a illustrates the rear region of electrode, and shows a gray rectangular band of inclined wall. The red rectangle in Figure 6a is separated into full sheets (Figure $6 \mathrm{~b}$ in order to view the 3D structure of the electrode intuitively. Figure $6 \mathrm{c}$ illustrates a cross-section image of the red line in Figure 6a, and shows a 2D profile of the electrode area. The depth of the electrode was approximately $11 \mu \mathrm{m}$, and the length of the base was approximately $16 \mu \mathrm{m}$; thus, we could calculate the angle of sloped wall to be approximately $40^{\circ}$. The depth of the electrode was determined by the etching time of PDMS and the initial height of the sacrificial posts. The mask pattern size for producing an electrode was $100 \mu \mathrm{m}$, but the measured size of the electrode was approximately $130 \mu \mathrm{m}$, which was extended by $30 \%$. These errors could be caused by underexposure, or approximate exposure, in photolithography, or the PDMS film could also be shrunk by NMP exposure during the PDMS etching. Therefore, in order to obtain an electrode of diameter $100 \mu \mathrm{m}$, a photomask of diameter $80 \mu \mathrm{m}$ should be used.
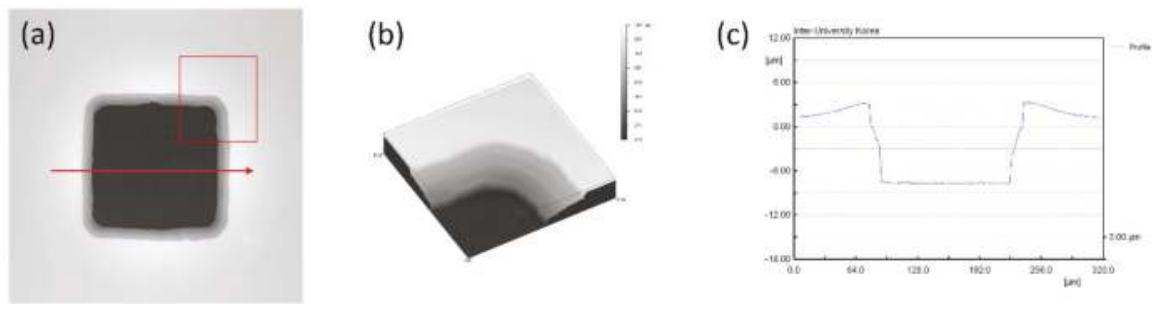

Figure 6. 3D rear profile images of plateau electrodes. (a,b) Wide field optical microscope images of an electrode. (a) The gray rectangle band shows the slope of the electrode. (b) An enlarged 3D projection of the region in the red rectangle of the image in (a). We can observe the gently sloping PDMS wall, which has an inclination angle of $40^{\circ}$. (c) 2D profile of the electrode obtained along the red line in (a). One side of the electrode is approximately $130 \mu \mathrm{m}$, and the depth of the electrode is approximately $11 \mu \mathrm{m}$.

Using this structure, a gold film could be stably deposited on the wall of PDMS holes via electron-gun equipment. Figure 7 shows enlarged images of the two types of electrode using an optical microscope and SEM. The gold electrode of the conventional recessed electrode was placed under the PDMS film in Figure 7a, and an image of the inclined PDMS hole is shown in Figure 7c. The gold was deposited along the gently sloped PDMS surface and the electrode was connected to the wire, as shown in Figure 7c. Moreover, we could observe a gray band on the edge of the electrode. When performing oxygen plasma treatment to increase the adhesion force between parylene with PDMS, parylene was slightly etched, resulting in the gray band was observed in SEM. This problem could be mitigated by decreasing the plasma processing time. 

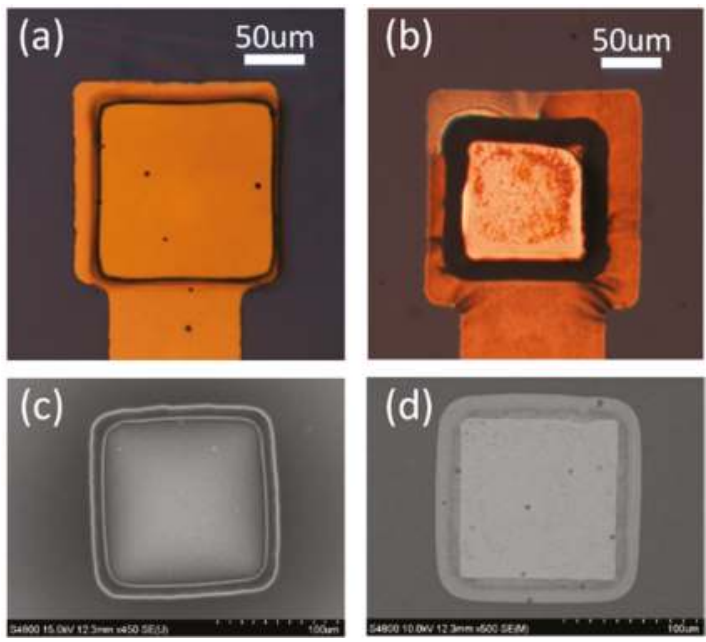

Figure 7. Optical and SEM images of a recessed electrode and a plateau electrode. (a) Enlarged image of the recessed electrode obtained using an optical microscope. Metal wires can be seen to pass under the PDMS film. (b) Plateau electrode. The dark band shows the PDMS slope connecting the electrode and wire. (c) SEM image of recessed electrode. The inclined holes can be observed on the PDMS film. (d) SEM image of plateau electrode.

\subsection{Surface Treatment}

The surface of PDMS was modified using oxygen plasma treatment and HEMA-grafting in an RIE system. HEMA was coated on oxygen-treated PDMS to facilitate the attachment of HEMA on the PDMS surface. The oxygen plasma-treated films demonstrated a very high degree of hydrophobic recovery, whereas HEMA coated onto oxygen-treated PDMS showed only a small decree of reconstruction [25]. To assess these properties, a gold thin film was deposited on the PDMS and the adhesion between the thin gold film and pretreated PDMS was tested by an adhesion test. The results from two different adhesive tapes are shown in Table 2.

Table 2. Results of adhesion test with tapes 1 and 2.

\begin{tabular}{ccccc}
\hline Method & $\begin{array}{c}\text { Time after } \\
\text { treatment (h) }\end{array}$ & $\begin{array}{c}\text { Adhesion test } \\
\text { with tape 1 }\end{array}$ & $\begin{array}{c}\text { Adhesion test } \\
\text { with tape 2 }\end{array}$ & $\begin{array}{c}\text { Contact angle } \\
\text { (degrees) }\end{array}$ \\
\hline No treatment & - & $\mathrm{X}$ & $\mathrm{X}$ & $\sim 105$ \\
\hline \multirow{2}{*}{ Oxygen plasma } & 1 & $0 \%$ & $0 \%$ & $\sim 10$ \\
& 24 & $60 \%$ & $80 \%$ & $\sim 92$ \\
\hline \multirow{2}{*}{ HEMA } & 1 & $0 \%$ & $0 \%$ & $\sim 12$ \\
& 48 & $0 \%$ & $0 \%$ & $\sim 20$ \\
& 120 & $0 \%$ & $0 \%$ & $\sim 35$ \\
\hline
\end{tabular}

After the oxygen plasma-treated PDMS was stored for $24 \mathrm{~h}$ at room temperature, both tests failed due to the reoccurrence of hydrophobicity. However, samples stored for only $1 \mathrm{~h}$ passed both tests. HEMA-treated PDMS samples were stored for $1 \mathrm{~h}, 24 \mathrm{~h}$, and 7 days in room temperature. In this case, all samples passed the gold adhesion test. Thus, HEMA processing maintained the hydrophilic surface of the PDMS for a long time, and facilitated the follow-up processes. 


\subsection{Electrode Impedance Measurement}

The equivalent circuit model of electrode and electrolyte interface consists of solution resistance, a constant phase element and the charge transfer resistance [30]. The constant phase element and the charge transfer resistance are connected in parallel and the solution resistance is connected in series. These factors are the theoretical values typically used to represent the electrode-electrolyte impedance [31]. The charge transfer resistance and constant phase element are factors influenced by the size and structure of the electrode, and the solution resistance is the resistance due to the solution between the working electrode and the counter electrode. It can be assumed that solution resistance and the constant phase element are the same because the fabricated electrodes have been exposed to the same process.

Figure 8 shows the average electrode impedance curves corresponding to the two types of electrodes. The average impedance values at $1 \mathrm{kHz}$ were $150 \mathrm{k} \Omega$ for the plateau type and $220 \mathrm{k} \Omega$ for the recessed type. This was close to the required impedance range for a single-unit recording neural probe [27,32]. The electrode size of the recessed type was $108 \mu \mathrm{m} \times 108 \mu \mathrm{m}$, and that of the plateau type was $130 \mu \mathrm{m} \times 130 \mu \mathrm{m}$. When the electrode is in contact with a living tissue composed of the electrolyte, an electrochemical double layer is formed between the electrode and the electrolyte, and this phenomenon can be quantified using impedance [8,9]. Therefore, the impedance largely depends on the area of the electrode; ignoring the material and deposition condition of the electrode, the impedance of the electrode is inversely proportional to the electrode area. The area of the plateau electrode was approximately 1.45 times larger than that of the recessed electrode, and the difference in the impedance values increased because the electrodes were located at a depth of $12 \mu \mathrm{m}$ from the surface. Assuming that the thickness and roughness of the gold were identical, the impedance difference between the two electrodes may be considered to be caused by the area and depth of the electrodes. If the area of recessed type electrode is converted into the same area to plateau electrode, the impedance of recessed electrode can be about $151.7 \mathrm{k} \Omega$. Therefore, it can be seen that an additional impedance of about $1.7 \mathrm{k} \Omega$ occurs due to the depth difference of about $12 \mu \mathrm{m}$. Because the impedance values are similar, the conductivity of the two MEA types can be considered to be similar to each other. However, in order to support this opinion, additional studies are required through the same electrode size and various recessed depths.

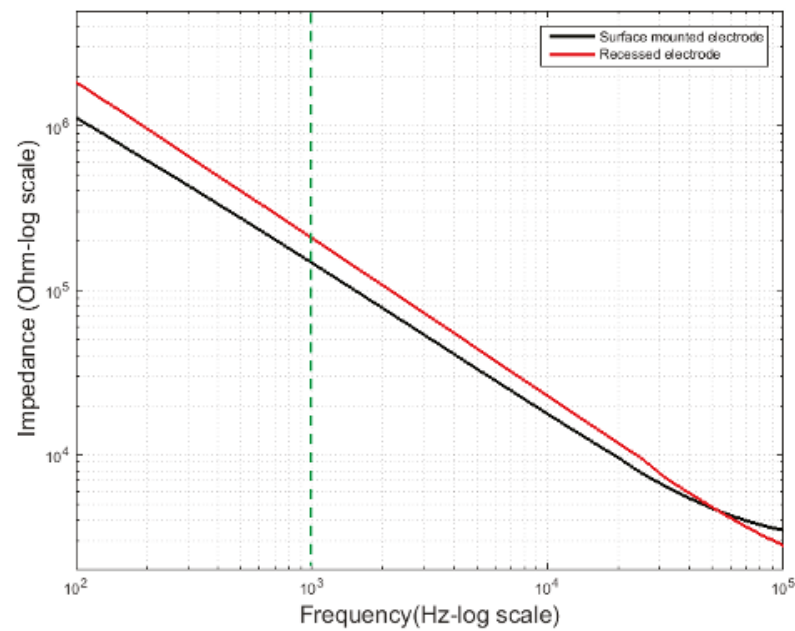

Figure 8. Electrode impedance measurement. Five measurements of electrode impedance on the eight individual electrodes of 10 MEAs are considered. The average electrode impedance curves rely on the electrode area and depth. 


\subsection{Surface Contact Performance}

We can observe the contact performance of the plateau electrode through the quality of interface between the electrode and a thin slide glass, as shown in Figure 9. An example of an air bubble trapped between the recessed electrode and a slide glass is shown in Figure 9b. Conventionally, the oxygen plasma treatment has been used to alleviate this trapped-air phenomenon. However, as previously described, surface modification via oxygen plasma is only a transient solution, and a new permanent scheme is necessary. There is no space to trap air in the case of the plateau electrode structure, as shown in Figure 9b. Thus, and it can not only be a permanent solution, but also improve the contact ability and signal recording performance. In addition, as the PDMS material exhibits high conformability and elasticity, so the performances of MEA can be further improved.
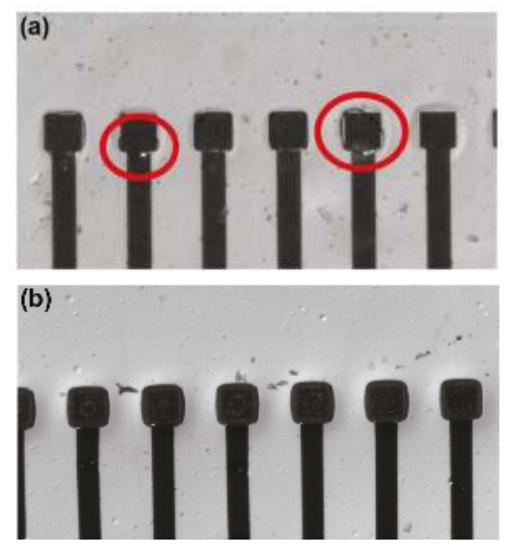

Figure 9. Optical microscope images for surface contact performance of plateau electrode. (a) An air bubble is trapped in the recessed electrode due to the hydrophobicity of the PDMS surface. (b) As there is no gap between the slide glass and electrodes, air bubbles are not seen on plateau electrodes.

\subsection{In Vivo Experiments}

The PDMS-based MEA was easily attached to the spinal cord, as shown in Figure 10a. Somatosensory evoked potentials (SSEPs) were observed as negative-first, biphasic responses after the stimulus on several channels in Figure 10c-e. Significant neural responses were recorded within the stimulus period. When using a recording electrode, the repopulation phenomenon is a very important factor in determining whether a biological signal can be recorded. In the spinal cord, which is a region without repopulation, we could not record a biological signal using recessed electrodes.

However, the plateau electrode could successfully record spinal cord signals. Figure 10a presents the recorded waveforms for $90 \mathrm{~s}$ from channel 7, and the red dotted line indicates the start and end of stimulus.

These experiments were conducted as proof-of-concept for the proposed fabrication. Future work can be to expand the fabrication method to various planar recording electrodes. 
(a)
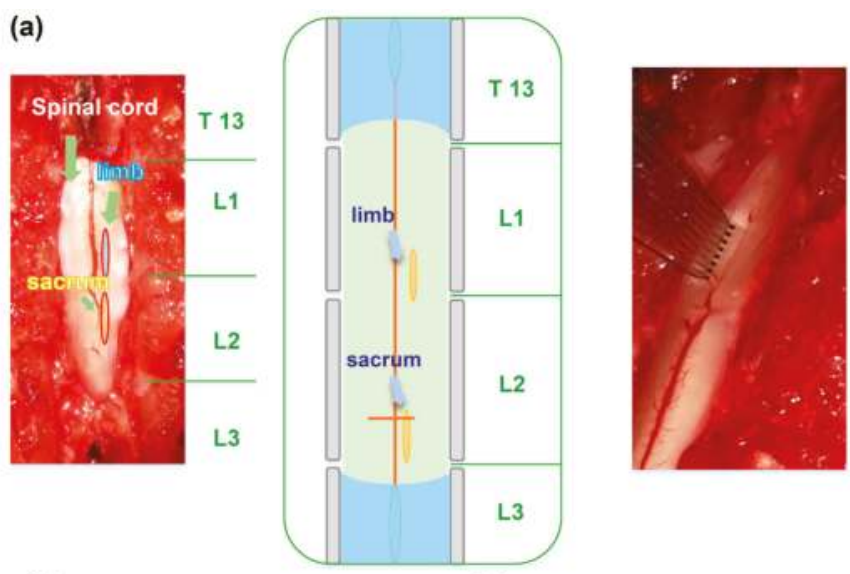

(b)

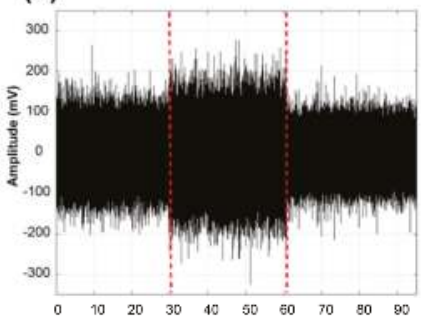

(c)
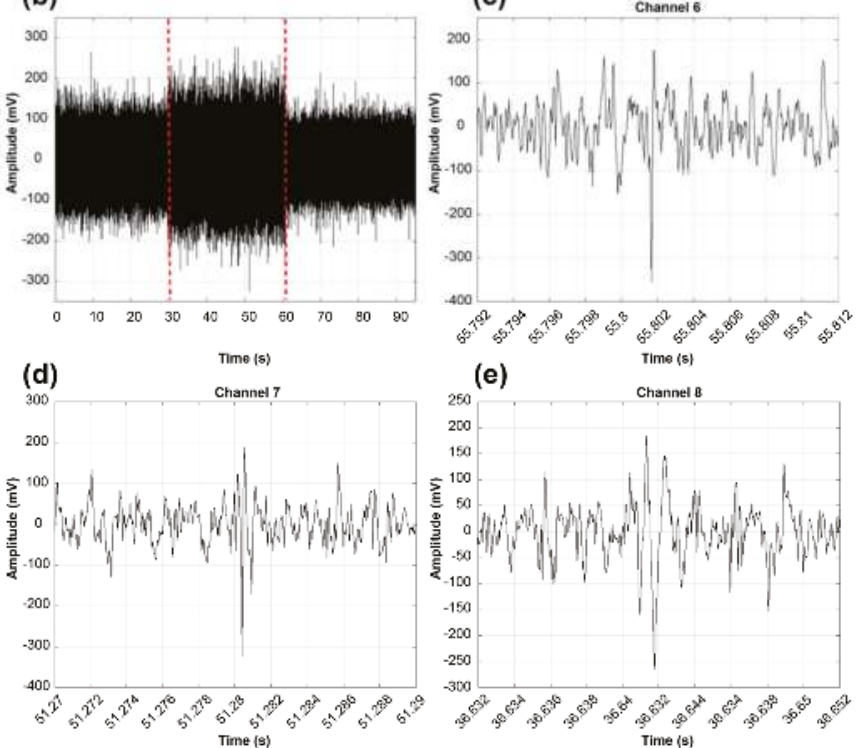

Figure 10. In vivo experiment. (a) In vivo placement of a PDMS-based MEA on a rat spinal cord. (b) Raw data from Channel 7. The period of the stimulus is indicated by the red dotted-lines. (c) An SSEPR from Channel 6. (d) An SSEPR from Channel 7. (e) An SSEPR from Channel 8.

\section{Conclusions}

A PDMS-based plateau MEA has been successfully fabricated and its mechanical and electrical characteristics have been demonstrated. The PDMS material is flexible, chemically inert, and has a similar Young's modulus as body tissues. It also satisfies properties that are necessary in the neural prosthesis field [18]. Moreover, the conformability and elasticity of PDMS are excellent compared to other polymers such as polyimide, parylene, and liquid crystal polymers (LCPs) $[18,19]$. Tests for cytotoxicity, extraction, and chronic toxicity have validated the biocompatibility of PDMS-based MEAs $[11,16]$. Recently, there is research to embed conductive materials such as Ag nanowires instead of depositing metal on PDMS, which have low surface energy and low bonding strength with other materials [33]. There is also research that utilizes the characteristics of PDMS with high elasticity, 
and use it as a touch sensor in a place with high movement [34]. A study has been done to fabricate transparent electrodes using the transparent characteristics of PDMS [35]. In this study we developed a method to fabricate electrode with surface-mounting structure by the molding technique. If we use this technique, a multilayer PDMS electrode can be developed, and the integration degree of the electrode can be increased. Therefore, we expect the PDMS-based plateau MEA to be an attractive alternative to conventional MEAs.

Two methods have been used widely for patterning metal films-wet-etching [12,17,20], and lift-off $[14,18]$. We have chosen the wet-etching method for its high resolution, stability, and yield of gold patterning. During a preliminary study of the lift-off method, we found that the photoresist film cracked during bake time due to a difference in the thermal expansion coefficients of PDMS and the photoresist. Photoresist cracking could be prevented by ensuring a slow temperature change of the sample, but in this case, a long period of time would be required to ensure that a reliable photoresist film is generated on the PDMS. To solve these problems, we performed photolithography on the sample after depositing metal on the PDMS layer. Thus, the metal deposited on the PDMS canceled out the thermal expansion of the PDMS and enabled patterning of the photoresist without cracks. While developing the photoresist, if the temperature of the developer is too low, the metal film could also become cracked when the photoresist cracks. Therefore, the developer (AZ MIF 300, AZ Electronic Material Corp., Branchburg, NJ, USA) should be used at approximately $30^{\circ}$. Furthermore, the adhesion between PDMS and metal was an important factor in achieving high resolution pattering. If the adhesion is low, the micro-pattern can be removed. By grafting HEMA onto PDMS, both the hydrophilicity retention time and adhesion force with the metal were improved.

The straight metal line on the PDMS substrate was easily damaged by tensile force, whereas a thin PDMS film can easily stretch. Serpentine-shaped metal patterns have been used to prevent metal line fractures caused by deformations and to provide stretchability upon tension or compression. Validation of the usage of serpentine metal lines was already performed successfully [32], and the results can be applied to the plateau electrode in order to solve the problem caused by tensile forces.

The impedance of the proposed plateau MEA was $150 \mathrm{k} \Omega$ at a frequency of $1 \mathrm{kHz}$. The proposed MEA was conformably placed over the spinal cord of a rat and neural activity was recorded. The conventional recessed MEAs could not record the neural signal due to the trapped air in the electrode. The proposed plateau electrode could record the neural signals reliably because the design prevents the air trapping phenomenon.

Acknowledgments: This work was supported by the National Research Foundation of Korea (NRF) grant funded by the Korean government (Ministry of Education) (No. NRF-2017R1D1A1B03028351).

Author Contributions: Conceptualization: Jun-Min Kim; Data curation: Jun-Min Kim; Formal analysis: Jun-Min Kim; Funding acquisition: Jun-Min Kim; Fabrication: Jun-Min Kim, Woo Ram Lee; Methodology: Jun-Min Kim; Project administration: Jun-Min Kim; Resources: Jun-Min Kim; Animal Experiment: Changkyun Im; Supervision: Jun-Min Kim; Validation: Jun-Min Kim; Visualization: Jun-Min Kim; Writing—original draft: Jun-Min Kim; and Writing-review \& editing: Jun-Min Kim.

Conflicts of Interest: The authors declare no conflict of interest.

\section{References}

1. Aravanis, A.M.; Wang, L.P.; Zhang, F.; Meltzer, L.A.; Mogri, M.Z.; Schneider, M.B.; Deisseroth, K. An optical neural interface: In vivo control of rodent motor cortex with integrated fiberoptic and optogenetic technology. J. Neural Eng. 2007, 4, S143-S156. [CrossRef] [PubMed]

2. Donoghue, J.P.; Nurmikko, A.; Black, M.; Hochberg, L.R. Assistive technology and robotic control using motor cortex ensemble-based neural interface systems in humans with tetraplegia. J. Physiol. 2007, 579, 603-611. [CrossRef] [PubMed]

3. Donoghue, J.P. Bridging the Brain to the World: A Perspective on Neural Interface Systems. Neuron 2008, 60, 511-521. [CrossRef] [PubMed] 
4. Disselhorst-Klug, C.; Bahm, J.; Ramaekers, V.; Trachterna, A.; Rau, G. Non-invasive approach of motor unit recording during muscle contractions in humans. Eur. J. Appl. Physiol. 2000, 83, 144-150. [CrossRef] [PubMed]

5. Reucher, H.; Rau, G.; Silny, J. Spatial Filtering of Noninvasive Multielectrode EMG: Part I-Introduction to Measuring Technique and Applications. IEEE Trans. Biomed. Eng. 1987, BME-34, 98-105. [CrossRef]

6. Rubehn, B.; Bosman, C.; Oostenveld, R.; Fries, P.; Stieglitz, T. A MEMS-based flexible multichannel ECoG-electrode array. J. Neural Eng. 2009, 6, 036003. [CrossRef] [PubMed]

7. Wagenaar, D.A.; Potter, S.M. Real-time multi-channel stimulus artifact suppression by local curve fitting. J. Neurosci. Meth. 2002, 120, 113-120. [CrossRef]

8. Wise, K.D.; Angell, J.B. A Low-Capacitance Multielectrode Probe for Use in Extracellular Neurophysiology. IEEE Trans. Biomed. Eng. 1975, BME-22, 212-219. [CrossRef]

9. Wise, K.D.; Angell, J.B.; Starr, A. An Integrated-Circuit Approach to Extracellular Microelectrodes. IEEE Trans. Biomed. Eng. 1970, BME-17, 238-247. [CrossRef]

10. He, W.; McConnell, G.C.; Schneider, T.M.; Bellamkonda, R.V. A Novel Anti-inflammatory Surface for Neural Electrodes. Adv. Mater. 2007, 19, 3529-3533. [CrossRef]

11. Berdondini, L.; Chiappalone, M.; van der Wal, P.D.; Imfeld, K.; de Rooij, N.F.; Koudelka-Hep, M.; Tedesco, M.; Martinoia, S.; van Pelt, J.; Le Masson, G.; et al. A microelectrode array (MEA) integrated with clustering structures for investigating in vitro neurodynamics in confined interconnected sub-populations of neurons. Sens. Actuators B 2006, 114, 530-541. [CrossRef]

12. Boppart, S.A.; Wheeler, B.C.; Wallace, C.S. A flexible perforated microelectrode array for extended neural recordings. IEEE Trans. Biomed. Eng. 1992, 39, 37-42. [CrossRef] [PubMed]

13. Cheung, K.C.; Renaud, P.; Tanila, H.; Djupsund, K. Flexible polyimide microelectrode array for in vivo recordings and current source density analysis. Biosens. Bioelectron. 2007, 22, 1783-1790. [CrossRef] [PubMed]

14. Rodger, D.C.; Fong, A.J.; Li, W.; Ameri, H.; Ahuja, A.K.; Gutierrez, C.; Lavrov, I.; Zhong, H.; Menon, P.R.; Meng, E.; et al. Flexible parylene-based multielectrode array technology for high-density neural stimulation and recording. Sens. Actuators B 2008, 132, 449-460. [CrossRef]

15. Kim, D.-H.; Viventi, J.; Amsden, J.J.; Xiao, J.; Vigeland, L.; Kim, Y.-S.; Blanco, J.A.; Panilaitis, B.; Frechette, E.S.; Contreras, D.; et al. Dissolvable films of silk fibroin for ultrathin conformal bio-integrated electronics. Nat. Mater. 2010, 9, 511-517. [CrossRef] [PubMed]

16. Chou, N.; Yoo, S.; Kim, S. A Largely Deformable Surface Type Neural Electrode Array Based on PDMS. IEEE Trans Neural Syst. Rehabil. 2013, 21, 544-553. [CrossRef] [PubMed]

17. Meacham, K.W.; Giuly, R.J.; Guo, L.; Hochman, S.; DeWeerth, S.P. A lithographically-patterned, elastic multi-electrode array for surface stimulation of the spinal cord. Biomed. Microdevices 2007, 10, 259-269. [CrossRef] [PubMed]

18. Guo, L.; Meacham, K.W.; Hochman, S.; DeWeerth, S.P. A PDMS-Based Conical-Well Microelectrode Array for Surface Stimulation and Recording of Neural Tissues. IEEE Trans. Biomed. Eng. 2010, 57, 2485-2494. [PubMed]

19. McClain, M.A.; Clements, I.P.; Shafer, R.H.; Bellamkonda, R.V.; LaPlaca, M.C.; Allen, M.G. Highly-compliant, microcable neuroelectrodes fabricated from thin-film gold and PDMS. Biomed. Microdevices 2011, 13, 361-373. [CrossRef] [PubMed]

20. Adrega, T.; Lacour, S.P. Stretchable gold conductors embedded in PDMS and patterned by photolithography: Fabrication and electromechanical characterization. J. Micromech. Microeng. 2010, 20, 055025. [CrossRef]

21. Kim, J.M.; Oh, D.R.; Sanchez, J.; Kim, S.H.; Seo, J.M. Fabrication of polydimethylsiloxane (PDMS)—Based multielectrode array for neural interface. In Proceedings of the 2013 35th Annual International Conference of the IEEE Engineering in Medicine and Biology Society (EMBC), Osaka, Japan, 3-7 July 2013; pp. 1716-1719.

22. Kim, J.M.; Seo, J.M. Fabrication of Polydimethylsiloxane (PDMS)-Based Flexible Electrode Array for Improving Tissue Contact. In Proceedings of the 6th European Conference of the International Federation for Medical and Biological Engineering, Dubrovnik, Croatia, 7-11 September 2014; Lacković, I., Vasic, D., Eds.; Springer International Publishing: Cham, Switzerland, 2015; pp. 341-344.

23. Schuettler, M.; Henle, C.; Ordonez, J.; Suaning, G.J.; Lovell, N.H.; Stieglitz, T. Patterning of Silicone Rubber for Micro-Electrode Array Fabrication. In Proceedings of the 3rd International IEEE/EMBS Conference on Neural Engineering (CNE '07), Kohala Coast, HI, USA, 2-5 May 2007; pp. 53-56. 
24. Balakrisnan, B.; Patil, S.; Smela, E. Patterning PDMS using a combination of wet and dry etching. J. Micromech. Microeng. 2009, 19, 047002. [CrossRef]

25. Bodas, D.S.; Khan-Malek, C. Fabrication of long-term hydrophilic surfaces of poly(dimethyl siloxane) using 2-hydroxy ethyl methacrylate. Sens. Actuators B 2007, 120, 719-723. [CrossRef]

26. Koschwanez, J.H.; Carlson, R.H.; Meldrum, D.R. Thin PDMS Films Using Long Spin Times or Tert-Butyl Alcohol as a Solvent. PLoS ONE 2009, 4, e4572. [CrossRef] [PubMed]

27. Byun, I.; Coleman, A.W.; Kim, B. Transfer of thin Au films to polydimethylsiloxane (PDMS) with reliable bonding using (3-mercaptopropyl)trimethoxysilane (MPTMS) as a molecular adhesive. J. Micromech. Microeng. 2013, 23, 085016. [CrossRef]

28. Lee, S.E.; Jun, S.B.; Lee, H.J.; Kim, J.; Lee, S.W.; Im, C.; Shin, H.C.; Chang, J.W.; Kim, S.J. A Flexible Depth Probe Using Liquid Crystal Polymer. IEEE Trans Biomed. Eng. 2012, 59, 2085-2094. [PubMed]

29. Lee, J.N.; Park, C.; Whitesides, G.M. Solvent Compatibility of Poly(dimethylsiloxane)-Based Microfluidic Devices. Anal. Chem. 2003, 75, 6544-6554. [CrossRef] [PubMed]

30. Franks, W.; Heer, F.; McKay, I.; Taschini, S.; Sunier, R.; Hagleitner, C.; Hierlemann, A.; Baltes, H. CMOS monolithic microelectrode array for stimulation and recording of natural neural networks. In Proceedings of the12th International Conference on Transducers, Solid-State Sensors, Actuators and Microsystems, Boston, MA, USA, 8-12 June 2003.

31. Grahame, D.C. Mathematical theory of the faradaic admittance. J. Electrochem. Soc. 1952, 99, 370C-385C. [CrossRef]

32. Jeong, J.; Hyun Bae, S.; Min, K.S.; Seo, J.-M.; Chung, H.; June Kim, S. A Miniaturized, Eye-Conformable, and Long-Term Reliable Retinal Prosthesis Using Monolithic Fabrication of Liquid Crystal Polymer (LCP). IEEE Trans. Biomed. Eng. 2015, 62, 982-989. [CrossRef] [PubMed]

33. Yao, S.; Myers, A.; Malhotra, A.; Lin, F.; Bozkurt, A.; Muth, J.; Zhu, Y. Wearable Hydration Sensor with Conformal Nanowire Electrodes. Adv. Healthc. Mater. 2017, 6, 1601159. [CrossRef] [PubMed]

34. Chen, X.; Song, U.; Chen, H.; Zhang, J.; Zhang, H. An ultrathin stretchable triboelectric nanogenerator with coplanar electrode for energy harvesting and gesture sensing. J. Mater. Chem. A 2017, 5, 12361-12368. [CrossRef]

35. Liu, H.S.; Pan, B.C.; Liou, G.S. Highly transparent AgNW/PDMS stretchable electrodes for elastomeric electrochromic devices. Nanoscale 2017, 9, 2633-2699. [CrossRef] [PubMed]

(C) 2017 by the authors. Licensee MDPI, Basel, Switzerland. This article is an open access article distributed under the terms and conditions of the Creative Commons Attribution (CC BY) license (http:/ / creativecommons.org/licenses/by/4.0/). 
Article

\title{
Quartz Microcrystal-Hybridized Organosilicone Encapsulant with Enhanced Optical and Thermal Performances
}

\author{
Xin Chen ${ }^{1,2, \dagger}$, Yancong Feng ${ }^{1,2,+}$, Xiao Wang ${ }^{1,2}$, En Li ${ }^{1,2}$, Yao Wang ${ }^{1,2}$, Lingling Shui ${ }^{1,2}$, \\ Hao Li ${ }^{1,2, *}$, Nan $\mathrm{Li}^{3,4}$ and Guofu Zhou ${ }^{1,2,3,4, *}$ \\ 1 Guangdong Provincial Key Laboratory of Optical Information Materials and Technology \& Institute of \\ Electronic Paper Displays, South China Academy of Advanced Optoelectronics, \\ South China Normal University, Guangzhou 510006, China; chenxinchauncey@163.com (X.C.); \\ fengyancong@m.scnu.edu.cn (Y.F.); wang2009xiao@163.com (X.W.); lien1016@163.com (E.L.); \\ wangyao@m.scnu.edu.cn (Y.W.); shuill@m.scnu.edu.cn (L.S.) \\ 2 National Center for International Research on Green Optoelectronics, South China Normal University, \\ Guangzhou 510006, China \\ 3 Shenzhen Guohua Optoelectronics Tech. Co. Ltd., Shenzhen 518110, China; nan.li@guohua-oet.com \\ 4 Academy of Shenzhen Guohua Optoelectronics, Shenzhen 518110, China \\ * Correspondence: haoli@scnu.edu.cn (H.L.); guofu.zhou@m.scnu.edu.cn (G.Z.); Tel.: +86-20-3931-4813 (H.L.) \\ $\dagger$ These authors contributed equally to this work.
}

Received: 30 November 2017; Accepted: 13 January 2018; Published: 16 January 2018

\begin{abstract}
Encapsulant is one determining factor underpinning the device lifetimes of organic optoelectronics. However, encapsulant seriously needs improvement in optical, thermal, and mechanical properties, especially to develop organic light emitting diodes. In this study, we prepared an in situ crosslinked organosilane composite containing benzyloxy and glycidyl-modified quartz microcrystal (mQMC) as high performance encapsulant. In the present work, methylphenylsilanediol (MPSD) was introduced as a novel crosslinker to impart appropriate structural strength. Along with increasing mQMC fillers, this organosilane system shows improved properties, such as refractive index, thermal stability, and storage modulus. Specifically, these hybridized mQMCs in the organosilane framework may facilitate an approximate two-fold increase $(0.238 \mathrm{~W} /(\mathrm{m} \cdot \mathrm{K}))$ in overall thermal conductivity at the determined concentration.
\end{abstract}

Keywords: organosilane; quartz microcrystal; encapsulant; refractive index; thermal conductivity

\section{Introduction}

In recent years, organic electronics have attracted more and more interest in a range of fields, e.g., display, sensor array, lighting panel, photovoltaic module and so on. As the first commercial application, organic light emitting diodes (OLEDs) reduce energy consumption and decrease device complexity compared to conventional liquid crystal displays [1]. However, their extremely sensitive components remarkably weaken device reliability. Naturally, encapsulant becomes one determining factor to provide hermetic protection for OLEDs. In this process, optical, thermal, mechanical, water, and oxygen barriers are involved in the specific demands of encapsulant performance [2].

As a frame material, epoxy is widely used as encapsulant due to its good adhesion, strong mechanical strength and rapid curing processing, but is still limited by poor moisture resistance, weak thermal stability, and a low refractive index [3-7]. On the contrary, organosilicone resin possesses a relatively high refractive index, strong moisture resistance, and good thermal stability, as well as good compatibility with glass substrate [8-12]. Therefore, organosilicone has gradually become a more suitable encapsulant candidate with high performance. 
However, the modification or formula regulation of frame material falls short of the demands for an excellent OLED encapsulant. Typically, the introduction of high polarization groups or atoms to the matrix [13-16]—such as phenyl group [8,17-22], sulfur [15], fluorine [12,17], phosphor [18], and nitrogen [18] — can effectively heighten the refractive index. On the other hand, some nanoparticles—e.g., $\mathrm{TiO}_{2}[19,23]$ and $\mathrm{ZrO}_{2}[3] —$ can be also introduced to achieve a desirable refractive index of encapsulant by hybridization. At the same time, the addition of similar nanoparticles-such as boron nitride nanosheets [7,24,25], graphene nanosheets [9,10], black phosphorus [26], ZnO [27], silicon carbide [28], core-shell $\mathrm{Ag@SiO} 2$ [29], and carbon nanotube [30]—will contribute significantly to the thermal conductivity of encapsulant materials.

In this work, we chose cheap and compatible quartz microcrystal (QMC) as filler and crosslinkers, and chose novel methylphenylsilanediol (MPSD) as molecular crosslinker, to construct a highly in situ crosslinked organosilicone network. Hereinto, surface modification of QMC will not only bring abundant phenyl groups to this organosilicone system for high refractive index, but also increase crosslinking density for good mechanical strength and thermal stability. Especially, high thermal conductivity of QMC [8 W/(m.K)] also facilitates overall thermal diffusion in the system. Moreover, MPSD will favor appropriate structural rigidity of organosilicone encapsulant.

\section{Materials and Methods}

\subsection{Materials}

Dichloromethylphenylsilane (98\%), 3-glycidyloxypropyl trimethoxysilane (97\%, GMS), vinyltrimethoxysilane ( $>98 \%$, VTMS), and methyldiethoxysilane ( $>98 \%$, MDES) were all provided by J\&K Chemical Technology (Beijing, China). Barium hydroxide monohydrate $\left(97 \%, \mathrm{Ba}(\mathrm{OH})_{2} \cdot \mathrm{H}_{2} \mathrm{O}\right)$ was purchased from HWRK Chemical (Beijing, China). Quartz microcrystal (mean size: $\sim 5 \mu \mathrm{m}$ ) was bought from Lianyungang Donghai Silica Powder Co., Ltd. (Jiangsu, China). Both Pt Karstedt's catalyst (Platinum(0)-1,3-divinyl-1,1,3,3-tetramethyldisiloxane complex solution in $p$-xylene $(\sim 2 \% \mathrm{Pt})$, Sigma-Aldrich, St. Louis, MO, USA) and Amberlite IRC76 (Alfa Aesar, Haverhill, MA, USA) were directly used. All other reagents and organic solvents are of analytic reagent (AR) without further treatment prior to use.

\subsection{Synthesis of MPSD}

MPSD was obtained by hydrolysis reaction (see Figure 1A) [31]. Typically, dichloromethylphenylsilane was dropwise added to the mixture of triethylamine, acetone, ultrapure water, and ether for $1 \mathrm{~h}$ while stirring at $0{ }^{\circ} \mathrm{C}$. Then, the redundant triethylamine hydrochloride was removed by vacuum filtration. Subsequently, the resulting filtrate was concentrated to one-tenth of the initial volume and then precipitated in excess cold $n$-hexane. After freezing for $24 \mathrm{~h}$, the final MPSD was separated out and collected by vacuum filtration.

\subsection{Modification of Quartz Microcrystal}

Both a determined amount of QMC and excess benzyl alcohol were added into a stainless vessel and then heated up to $240^{\circ} \mathrm{C}$ for $96 \mathrm{~h}$. Afterwards, benzyloxy-modified QMCs were obtained by high speed centrifugation for $10 \mathrm{~min}$. Next, these QMCs were mixed fully with GMS in tetrahydrofuran under sonication for $2 \mathrm{~h}$. Finally, all the products, benzyloxy, and glycidyl-modified QMC (mQMC) were concentrated to $5 \mathrm{~mL}$ in dry argon flow (see Figure 1B) [3].

\subsection{Synthesis of Vinylmethylphenylsiloxane Resin (VMPS)}

VMPS was obtained by sol-gel condensation of VTMS and MPSD (molar ratio: 1:1) in $p$-xylene (10 wt. \%) at $80^{\circ} \mathrm{C}$ while stirring for $4 \mathrm{~h}$, using $\mathrm{Ba}(\mathrm{OH})_{2} \cdot \mathrm{H}_{2} \mathrm{O}(0.1 \mathrm{~mol} \%)$ as catalyst (see Figure 1C) [32]. After the reaction proceeded for $2 \mathrm{~h}$, the catalyst was filtered out with a $10 \mu \mathrm{m}$ syringe filter. 
The dropwise addition of $\mathrm{mQMC}$ into the reacting mixture followed immediately. Finally, all the volatile components were removed in vacuum.

\subsection{Synthesis of Methylphenylsiloxane Resin (MPS)}

Similarly, MPS was synthesized by sol-gel condensation of MDES and MPSD (molar ratio: 1:1.5) at $100{ }^{\circ} \mathrm{C}$ while stirring for $12 \mathrm{~h}$, using Amberlite IRC76 (0.1 mol \%) as acidic catalyst (see Figure 1C) [32]. Once the reaction proceeded for $2 \mathrm{~h}$, the catalyst was filtered out with a $10 \mu \mathrm{m}$ syringe filter. Then the dropwise addition of $\mathrm{mQMC}$ into the reacting mixture followed immediately. Finally, all the volatile components were removed in vacuum.

\subsection{Fabrication of Quartz-Organosilicone Composite}

The quartz-organosilicone composite was synthesized by hydrosilylation of VMPS and MPS using Pt Karstedt's catalyst while stirring for $2 \mathrm{~h}$ (see Figure 1D) [32]. Afterwards, the mixture was homogenized by sonication for $1 \mathrm{~h}$ and then cast into a customized stainless steel mold. Finally, the sample was cured at $90^{\circ} \mathrm{C}$ for $1 \mathrm{~h}$, followed by heating at $150{ }^{\circ} \mathrm{C}$ for $2 \mathrm{~h}$ in air.

\subsection{Chemical Characterizations}

The chemical structure of MPSD was tested by electron ionization mass spectrometer (EI-MS) using a NexION 300 supplied by PerkinElmer (Waltham, MA, USA). The surface modification of QMC was identified by Fourier transform infrared spectrometer (FTIR) using a Vertex 70 supplied by Bruker (Karlsruhe, Germany) in a spectral range of $4000-400 \mathrm{~cm}^{-1}$ with the resolution of $2 \mathrm{~cm}^{-1}$. Samples were prepared using $\mathrm{KBr}$ plate method.

\subsection{Optical Measurements}

The transmittances of the final quartz-organosilicone composite films were measured by ultraviolet-visible-near infrared spectrophotometer (UV-vis-NIR) using a LAMBDA 950 supplied by PerkinElmer in the wavelength range of 400-700 nm. All the thicknesses of the samples were $1 \mathrm{~mm}$. The measurements of refractive index were performed by refractometer using a DR-2 supplied by ATAGO (Tokyo, Japan) at $589 \mathrm{~nm}$ and $25^{\circ} \mathrm{C}$.

\subsection{Thermal Analysis}

The thermal stability of the samples was examined by thermal gravimetric analysis (TGA) using a TGA 2 supplied by METTLER TOLEDO (Greifensee, Switzerland) in the temperature range from $25^{\circ} \mathrm{C}$ to $1000{ }^{\circ} \mathrm{C}$ with a heating rate of $10^{\circ} \mathrm{C} / \mathrm{min}$ in nitrogen atmosphere (flow rate: $50 \mathrm{~mL} / \mathrm{min}$ ).

The thermal conductivity was determined by laser thermal conductivity analysis using a LFA447 supplied by NETZSCH (Selb, Germany) at $25^{\circ} \mathrm{C}$. All the sizes of samples were $10 \times 10 \mathrm{~mm}^{2}$ with the thickness of $1 \mathrm{~mm}$.

\subsection{Mechanical Test}

The mechanical performance was tested by dynamic mechanical thermal analysis (DMA) using a DMA 1 supplied by METTLER TOLEDO (Greifensee, Switzerland) in a temperature range from $50^{\circ} \mathrm{C}$ to $260{ }^{\circ} \mathrm{C}$ with a heating rate of $5^{\circ} \mathrm{C} / \mathrm{min}$ at $1 \mathrm{~Hz}$ in the shear mode.

\section{Results and Discussion}

As the best candidate for encapsulant, organosilicone still needs to be improved in optical and thermal performances. In the present work, both MPSD crosslinker and QMC additive were introduced into the siloxane matrix by four-step fabrication (see Figure 1). The related descriptions of fabrication are presented in the Materials and Methods section and Supporting Information in detail. MPSD was obtained by hydrolysis reaction as shown in Figure 1A. Hereinto MPSD is so active that reacts to form 
a series of different oligomers. As shown in Figure 2, there are a dimer peak at $288 \mathrm{~m} / \mathrm{z}$, and a trimer peak at $424 \mathrm{~m} / \mathrm{z}$, much stronger than the monomer peak at $154 \mathrm{~m} / \mathrm{z}$. Although the obtained product is a mixture of MPSD and its oligomers, their good solubility in two silane monomers-VTMS and MDES-contribute greatly to the following coupling reactions.

A<smiles>C[Si](Cl)(Cl)c1ccccc1</smiles>

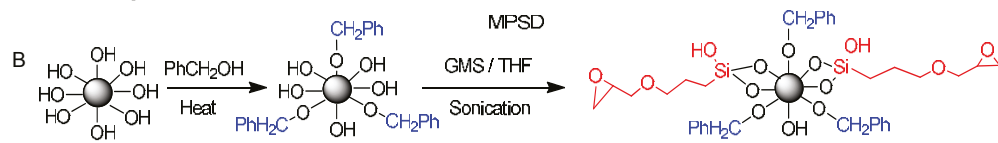

C
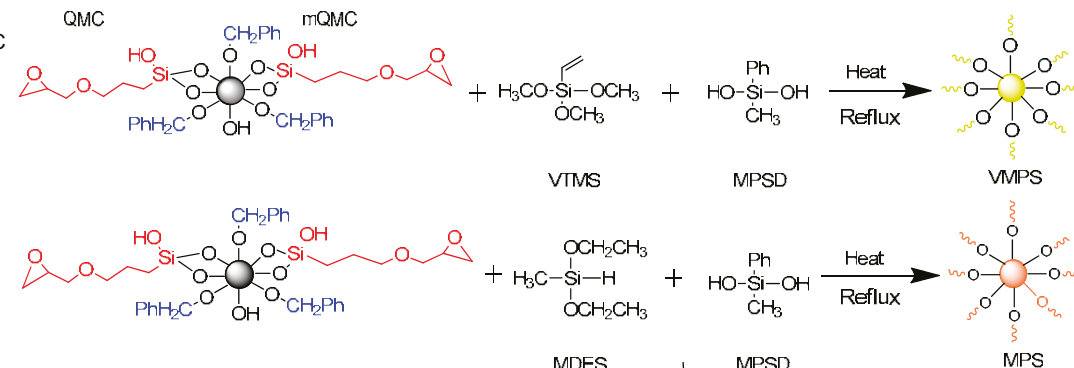

$\mathrm{D}$
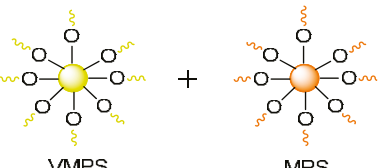

MDES

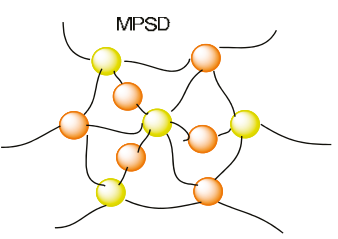

Figure 1. Synthesis routes of (A) MPSD; (B) surface-modified QMC; (C) two intermediate resins_VMPS and MPS; and (D) final quartz-organosilane composite.

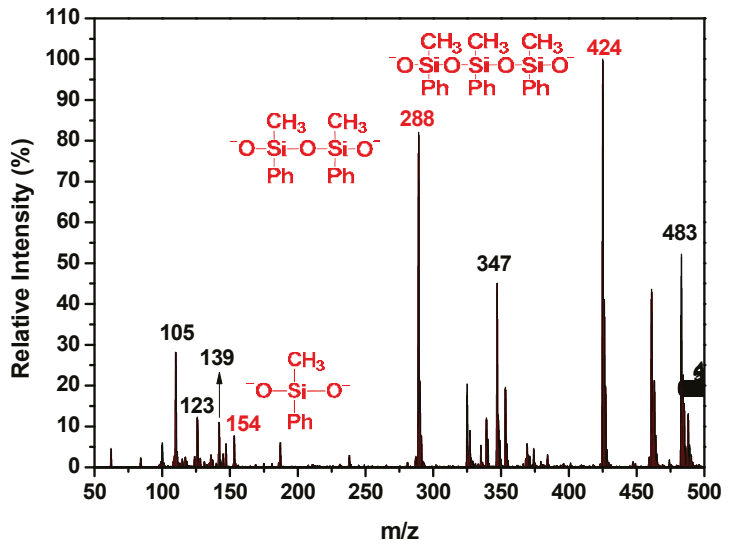

Figure 2. Mass spectrum of MPSD. The related peaks list below: $105(m / z, \mathrm{SiPh}), 123(\mathrm{~m} / z, \mathrm{SiOPh})$, $139\left(m / z, \mathrm{Si}(\mathrm{OH})_{2} \mathrm{Ph}\right), 154\left(m / z, \mathrm{SiO}_{2} \mathrm{PhCH}_{3}\right), 288\left(m / z, \mathrm{Si}_{2} \mathrm{O}_{3} \mathrm{Ph}_{2} \mathrm{C}_{2} \mathrm{H}_{6}\right), 347\left(m / z, \mathrm{Si}_{3} \mathrm{O}_{4} \mathrm{Ph}_{2} \mathrm{C}_{3} \mathrm{H}_{9}\right)$, $424\left(m / z, \mathrm{Si}_{3} \mathrm{O}_{4} \mathrm{Ph}_{3} \mathrm{C}_{3} \mathrm{H}_{9}\right), 483\left(m / z, \mathrm{Si}_{4} \mathrm{O}_{5} \mathrm{Ph}_{3} \mathrm{C}_{4} \mathrm{H}_{12}\right)$. 
In fact, benzyloxy- and glycidyl-modified QMC (mQMC) (see Figure 1B) also act as another kind of crosslinker with extremely high crosslinking intensity. Figure 3 obviously displays the absorption bands of benzyl groups at $1400-1600 \mathrm{~cm}^{-1}$ [3]. This proves that the benzyl group is successfully grafted to the surface of QMC. It may effectively enhance the compatibility of QMC with the organosilicone frameworks, owing to similar chemical groups.

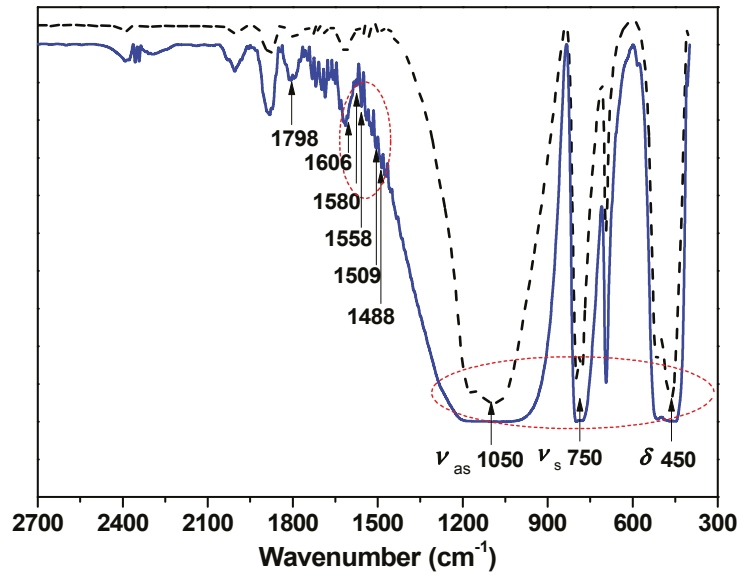

Figure 3. FTIR spectra of quartzes before and after surface modification with benzyl alcohol. The dash black line is quartz without modification and the solid blue line is the modified quartz. The related peaks list below: $450 \mathrm{~cm}^{-1}\left(\delta,-\mathrm{Si}-\mathrm{O}\right.$ linked with quartz microcrystal), $750 \mathrm{~cm}^{-1}\left(v_{\mathrm{s}},-\mathrm{Si}-\mathrm{O}\right.$ linked with quartz microcrystal), $1050 \mathrm{~cm}^{-1}$ ( $v_{\mathrm{as}},-\mathrm{Si}-\mathrm{O}$ linked with quartz microcrystal), $1488 \mathrm{~cm}^{-1}, 1509 \mathrm{~cm}^{-1}$, $1558 \mathrm{~cm}^{-1}, 1580 \mathrm{~cm}^{-1}, 1606 \mathrm{~cm}^{-1}\left(-\mathrm{C}_{6} \mathrm{H}_{5}\right.$ linked with benzyl alcohol), $1798 \mathrm{~cm}^{-1}$ (solvent THF).

At the same time, abundant glycidyl groups on the surface of mQMC-joined together with $\mathrm{Si}-\mathrm{H}$ functional bonds, vinyl groups, and MPSD—constructed a highly crosslinked network. Naturally, this predominant organosilicone nature will assign good optical properties to the quartz composites. As shown in Figure 4, transmittance of the neat organosilicone reaches nearly $90 \%$ in the visible range from $450 \mathrm{~nm}$ to $700 \mathrm{~nm}$. However, the high refractive index of mQMC is adverse to transparent blank matrix. Thus, along with increasing mQMC content, the composite exhibits a decreasing transmittance in the range of visible light.

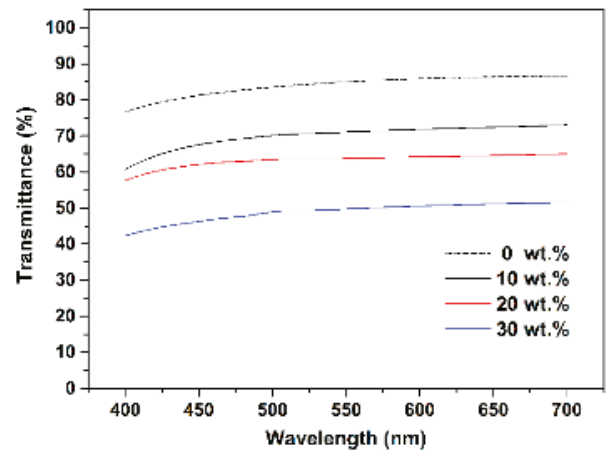

Figure 4. Transmittance curves of quartz-organosilicone composites with different mQMC contents in the visible spectrum. 
Contrary to the falling transmittance, the refractive index of quartz-organosilicone composite rises from initial 1.613 to 1.623 at the loading of $30 \mathrm{wt}$. \%. Surprisingly, the refractive index of the neat sample reaches a very high level of over 1.6, which is much larger than that of siloxane-hybridized materials with high refractive index in the previously reported works $[8,20]$. This performance may mainly attribute to the crosslinker we selected. According to the classic electromagnetic theory, the refractive index is derived from Lorentz-Lorenz equation [33]

$$
P=\frac{n^{2}-1}{n^{2}+1} \times \frac{M}{\rho}
$$

where $P$ is the molar polarization, $n$ is the refractive index of quartz-organosilicone, $M$ is the molecular weight, and $\rho$ is the density. Obviously, the refractive index has a positive correlation with the material polarization. As the key crosslinker, MPSD gives this framework plenty of benzyl groups to improve material polarization, which explains the extremely high refractive index of blank sample. On the other hand, as the content of filler increases, more and more benzyl groups of mQMC are introduced into the composite. Of course, high refractive index of mQMCs themselves is also a favorable factor. As revealed in Figure 5, the refractive index shows a good liner relation with the content of mQMC. It agrees well with the idea that the chemical structure is a critical factor in enhancing the refractive index of materials from the formula above [33].

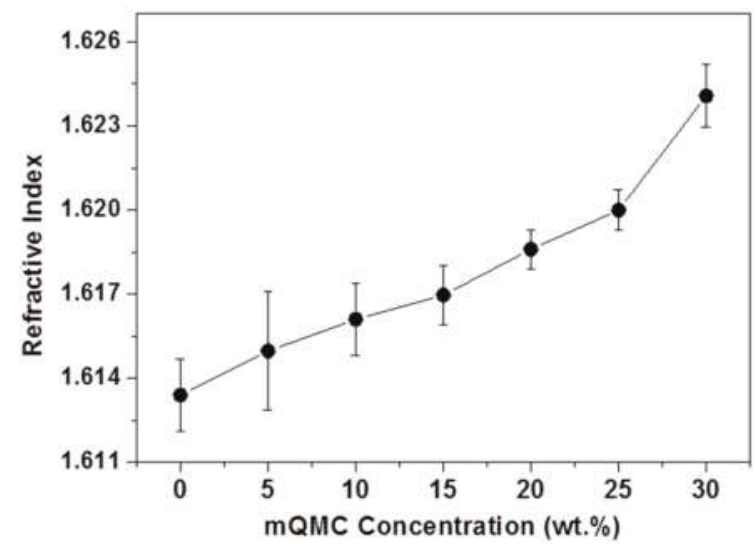

Figure 5. Refractive indexes of quartz-organosilicone composites with different mQMC contents.

Afterwards, the thermal performances of this highly crosslinked organosilicone network, including thermal stability and conductivity, are evaluated in detail. Figure 6 firstly exhibits high thermal stability of quartz-organosilicone composites. The derivatives of thermal spectra are presented in Figure S1. In Figure 6, for blank sample, 5\% weight loss temperature is $356{ }^{\circ} \mathrm{C}$, much higher than the common encapsulants [6]. As the content of mQMC filler rises above $20 \mathrm{wt}$. \%, a 5\% weight loss temperature develops from $356{ }^{\circ} \mathrm{C}\left(0 \mathrm{wt}\right.$. \%) to $361{ }^{\circ} \mathrm{C}(25 \mathrm{wt}$. \% and $30 \mathrm{wt}$. \%). This is different from the $\mathrm{ZrO}_{2}$ particle which is able to disturb the crosslinking network [3]. 


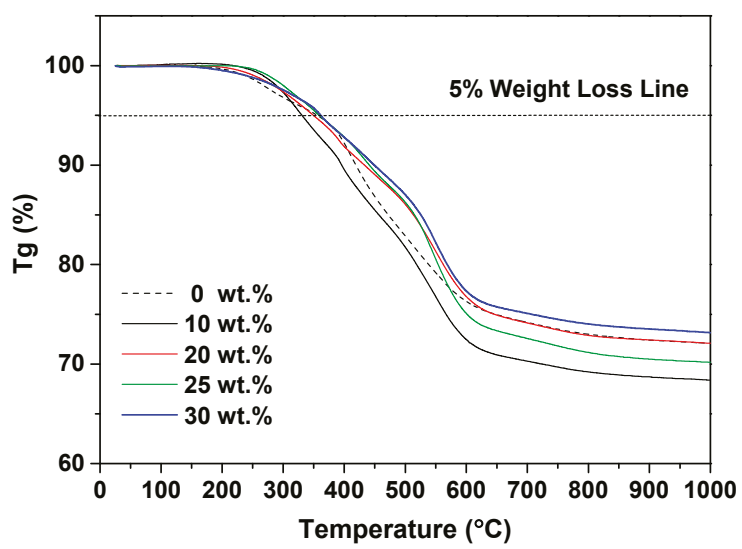

Figure 6. Thermogravimetric curves of quartz-organosilicone composites with different mQMC contents.

Except thermal stability, heat diffusion is a serious problem for encapsulant which influences the performance of electronic devices even the lifetime. For improving thermal conductivity of encapsulant, QMC (about $8 \mathrm{~W} /(\mathrm{m} \cdot \mathrm{K})$ ) is specifically chosen as the critical filler in this composite. Figure 7 illustrates that, the thermal conductivity of quartz-organosilicone composite reaches to the peak at the mQMC content of $25 \mathrm{wt}$. \%. It may be ascribed to the distribution of highly thermoconductive QMC in the organosilicone network. At the low concentration, mQMCs are well-distributed in the matrix to make the heat flux homogeneous. Here, the enhancement of thermal conductivity is obvious. However, once the mass ratio of $\mathrm{mQMC}$ filler achieves $30 \mathrm{wt}$ \%, the aggregation of particles emerges, leading to a decrease of thermal conductivity.

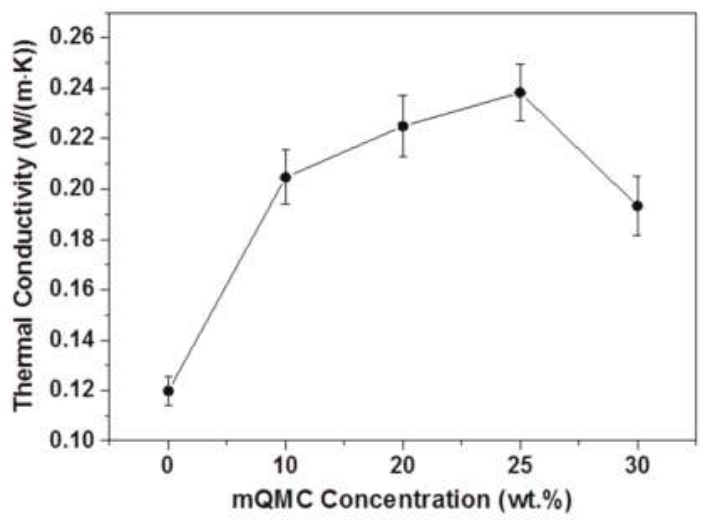

Figure 7. Thermal conductivities of quartz-organosilicone composites with different mQMC contents.

The last key parameter, the mechanical performances of quartz-organosilicone encapsulants, are represented in Figure 8. Remarkably, the highest storage modulus is obtained at the mQMC content of $30 \mathrm{wt}$. \%. As the mQMC content below $30 \mathrm{wt}$. \%, the addition of fillers does not bring any obvious variation of storage modulus. Once the content of filler reaches $30 \mathrm{wt}$. \%, the crosslinking network has formed and thus a sharp increment of storage modulus emerges, presenting an obvious reinforcing effect. Meanwhile, the TGA curves in Figure 6 also indicate that, the $30 \mathrm{wt}$. \% loading is enough to form a dense crosslinking network. Furthermore, the high storage modulus reveals that 
this kind of crosslinking quartz-organosilicone composite is strong enough to resist outer pressure during encapsulation.

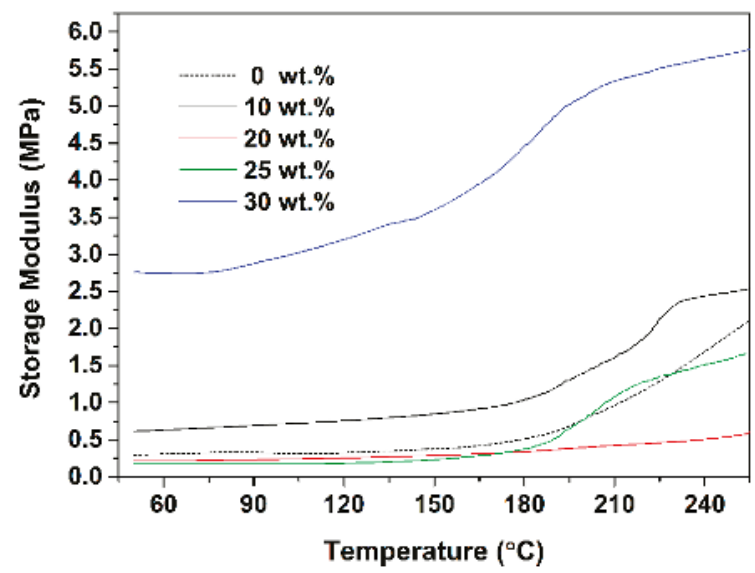

Figure 8. Dynamic mechanical thermal analyses of quartz-organosilicone composites with different mQMC contents in shear mode.

\section{Conclusions}

In this study, both compatible QMC and novel crosslinker MPSD were introduced into one in situ crosslinked organosilane matrix as high performance encapsulant. In this case, surface modified QMC as high density crosslinks can greatly increase the crosslinking degree of the organosilane composite to improve thermal stability and mechanical performance. Their high thermal conductivity also facilitates the overall thermal diffusion in the system with about double the coefficient of heat conductivity $(0.238 \mathrm{~W} /(\mathrm{m} \cdot \mathrm{K}))$. The only drawback is the falling transmittance along with increasing mQMC feeds. Moreover, MPSD possesses a more appropriate molecular rigidity compared with common diphenyl silanediol and dimethyl silanediol, so that it can integrate numerous phenyls on the surface of QMC into the organosilane encapsulant to create a very high refractive index ( 1.623) and greatly enhance storage modulus. In view of cost, performance, and system compatibility, this highly crosslinked quartz-organosilane composite is anticipated to be a good encapsulant candidate for improving reliability of OLEDs.

Supplementary Materials: The following are available online at www.mdpi.com/2073-4360/10/1/84/s1, Figure S1: Thermogravimetric curves of quartz-organosilicone composites with different mQMC contents. (a) 0 wt.\%, (b) 10 wt. \%, (c) 20 wt.\%, (d) 25 wt.\% and (e) 30 wt.\%.

Acknowledgments: This work was financially supported by the National Key Research and Development Program of China (No. 2016YFB0401501), National Natural Science Foundation of China (51561135014, U1501244), Guangdong Innovative Research Team Program (No. 2013C102), Guangdong Province Major Science and Technology Projects (No. 2015B090913004), Special Fund on Public Interest Research and Capacity Building in Guangdong Province (No. 2016A010101023), Key Cultivation Project of Basic Research of Guangdong Natural Science Foundation (No. 2014A030308013), Guangdong Provincial Key Laboratory of Optical Information Materials and Technology (No. 2017B030301007), MOE International Laboratory for Optical Information Technologies, and the 111 Project.

Author Contributions: Hao Li conceived and designed the experiments; Xin Chen, Xiao Wang, and En Li performed the experiments; Yancong Feng analyzed the data and made the figures; Lingling Shui, Yao Wang, and Nan Li provided some suggestions for this project; Hao Li, Yancong Feng, and Xin Chen wrote the manuscript; Guofu Zhou helped revise the manuscript. All authors critically reviewed the manuscript.

Conflicts of Interest: The authors declare no conflict of interest. 


\section{References}

1. De Mello, J.; Anthony, J.; Lee, S. Organic electronics: Recent developments. Chemphyschem 2015, 16, 1099-1100. [CrossRef] [PubMed]

2. Thejo Kalyani, N.; Dhoble, S.J. Novel materials for fabrication and encapsulation of OLEDs. Renew. Sustain. Energy Rev. 2015, 44, 319-347. [CrossRef]

3. Tao, P.; Li, Y.; Siegel, R.W.; Schadler, L.S. Transparent dispensible high-refractive index $\mathrm{ZrO}_{2} /$ epoxy nanocomposites for LED encapsulation. J. Appl. Polym. Sci. 2013, 130, 3785-3793. [CrossRef]

4. Li, T.; Zhang, J.; Wang, H.; Hu, Z.; Yu, Y. High-performance light-emitting diodes encapsulated with silica-filled epoxy materials. ACS Appl. Mater. Interfaces 2013, 5, 8968-8981. [CrossRef] [PubMed]

5. Yang, S.; Kwak, S.-Y.; Jin, J.; Kim, J.-S.; Choi, Y.; Paik, K.-W.; Bae, B.-S. Thermally resistant UV-curable epoxy-siloxane hybrid materials for light emitting diode (LED) encapsulation. J. Mater. Chem. 2012, 22, 8874-8880. [CrossRef]

6. Chen, Z.; Liu, Z.; Shen, G.; Wen, R.; Lv, J.; Huo, J.; Yu, Y. Effect of chain flexibility of epoxy encapsulants on the performance and reliability of light-emitting diodes. Ind. Eng. Chem. Res. 2016, 55, 7635-7645. [CrossRef]

7. Chen, J.; Huang, X.; Zhu, Y.; Jiang, P. Cellulose nanofiber supported 3D interconnected BN nanosheets for epoxy nanocomposites with ultrahigh thermal management capability. Adv. Funct. Mater. 2017, 27, 1604754. [CrossRef]

8. Kim, J.-S.; Yang, S.; Bae, B.-S. Thermally stable transparent sol-gel based siloxane hybrid material with high refractive index for light emitting diode (LED) encapsulation. Chem. Mater. 2010, 22, 3549-3555. [CrossRef]

9. Lee, S.; Hong, J.Y.; Jang, J. Multifunctional graphene sheets embedded in silicone encapsulant for superior performance of light-emitting diodes. ACS Nano 2013, 7, 5784-5790. [CrossRef] [PubMed]

10. Varenik, M.; Nadiv, R.; Levy, I.; Vasilyev, G.; Regev, O. Breaking through the solid/liquid processability barrier: Thermal conductivity and rheology in hybrid graphene-graphite polymer composites. ACS Appl. Mater. Interfaces 2017, 9, 7556-7564. [CrossRef] [PubMed]

11. Bartlett, M.D.; Kazem, N.; Powell-Palm, M.J.; Huang, X.; Sun, W.; Malen, J.A.; Majidi, C. High thermal conductivity in soft elastomers with elongated liquid metal inclusions. Proc. Natl. Acad. Sci. USA 2017, 114, 2143-2148. [CrossRef] [PubMed]

12. Bae, J.-Y.; Kim, H.-Y.; Lim, Y.-W.; Kim, Y.-H.; Bae, B.-S. Optically recoverable, deep ultraviolet (UV) stable and transparent sol-gel fluoro siloxane hybrid material for a UV LED encapsulant. RSC Adv. 2016, 6, 26826-26834. [CrossRef]

13. Chen, M.; Zhang, G.Y.; Liang, X.; Zhang, W.S.; Zhou, L.; He, B.F.; Song, P.; Yuan, X.; Zhang, C.H.; Zhang, L.Y.; et al. Thermally stable transparent sol-gel based active siloxane-oligomer materials with tunable high refractive index and dual reactive groups. RSC Adv. 2016, 6, 70825-70831. [CrossRef]

14. Kleine, T.S.; Nguyen, N.A.; Anderson, L.E.; Namnabat, S.; LaVilla, E.A.; Showghi, S.A.; Dirlam, P.T.; Arrington, C.B.; Manchester, M.S.; Schwiegerling, J.; et al. High refractive index copolymers with improved thermomechanical properties via the inverse vulcanization of sulfur and 1,3,5-triisopropenylbenzene. ACS Macro Lett. 2016, 5, 1152-1156. [CrossRef]

15. Sun, Z.; Huang, H.; Li, L.; Liu, L.; Chen, Y. Polythioamides of high refractive index by direct polymerization of aliphatic primary diamines in the presence of elemental sulfur. Macromolecules 2017, 50, 8505-8511. [CrossRef]

16. Kim, Y.H.; Kim, H.; Yeo, H.; Goh, M.; Ku, B.-C.; Hahn, J.R.; You, N.-H. Preparation of UV-curable acryl resin for high refractive index based on 1,5-bis(2-acryloylenethyl)-3,4-ethylenedithiothiophene. Eur. Polym. J. 2016, 75, 303-309. [CrossRef]

17. Nakabayashi, K.; Imai, T.; Fu, M.-C.; Ando, S.; Higashihara, T.; Ueda, M. Poly(phenylene thioether)s with fluorene-based cardo structure toward high transparency, high refractive index, and low birefringence. Macromolecules 2016, 49, 5849-5856. [CrossRef]

18. Hsu, C.-Y.; Han, W.-G.; Chiang, S.-J.; Su, W.-C.; Liu, Y.-L. Multi-functional branched polysiloxanes polymers for high refractive index and flame retardant LED encapsulants. RSC Adv. 2016, 6, 4377-4381. [CrossRef]

19. Liu, Y.; Lin, Z.Y.; Moon, K.-S.; Yoo, S.; Choi, J.; Wong, P. High refractive index and transparency nanocomposites as encapsulant for high brightness LED packing. Electron. Compon. Technol. Conf. 2013, 4, 553-556. 
20. Macdonald, E.K.; Lacey, J.C.; Ogura, I.; Shaver, M.P. Aromatic polyphosphonates as high refractive index polymers. Eur. Polym. J. 2017, 87, 14-23. [CrossRef]

21. Chung, P.T.; Chiou, S.H.; Tseng, C.Y.; Chiang, A.S. Preparation and evaluation of a zirconia/oligosiloxane nanocomposite for LED encapsulation. ACS Appl. Mater. Interfaces 2016, 8, 9986-9993. [CrossRef] [PubMed]

22. Kim, Y.H.; Lim, Y.-W.; Lee, D.; Kim, Y.H.; Bae, B.-S. A highly adhesive siloxane LED encapsulant optimized for high thermal stability and optical efficiency. J. Mater. Chem. C 2016, 4, 10791-10796. [CrossRef]

23. Ireni, N.G.; Karuppaiah, M.; Narayan, R.; Raju, K.V.S.N.; Basak, P. $\mathrm{TiO}_{2}$ / poly(thiourethane-urethane)-urea nanocomposites: Anticorrosion materials with NIR-reflectivity and high refractive index. Polymer 2017, 119, 142-151. [CrossRef]

24. Wang, F.; Zeng, X.; Yao, Y.; Sun, R.; Xu, J.; Wong, C.P. Silver nanoparticle-deposited boron nitride nanosheets as fillers for polymeric composites with high thermal conductivity. Sci. Rep. 2016, 6, 19394. [CrossRef] [PubMed]

25. Wang, F.; Zeng, X.; Yao, Y.; Gong, Z.; Wang, F.; Sun, R.; Xu, J.; Wong, C.P. Ice-templated assembly strategy to construct 3D boron nitride nanosheet networks in polymer composites for thermal conductivity improvement. Small 2015, 11, 6205-6213.

26. Zhu, J.; Park, H.; Chen, J.-Y.; Gu, X.; Zhang, H.; Karthikeyan, S.; Wendel, N.; Campbell, S.A.; Dawber, M.; $\mathrm{Du}, \mathrm{X}$; et al. Revealing the origins of $3 \mathrm{D}$ anisotropic thermal conductivities of black phosphorus. Adv. Electron. Mater. 2016, 2, 1600040. [CrossRef]

27. Wang, Z.; Lu, Z.; Mahoney, C.; Yan, J.; Ferebee, R.; Luo, D.; Matyjaszewski, K.; Bockstaller, M.R. Transparent and high refractive index thermoplastic polymer glasses using evaporative ligand exchange of hybrid particle fillers. ACS Appl. Mater. Interfaces 2017, 9, 7515-7522. [CrossRef] [PubMed]

28. Yao, Y.; Zeng, X.; Pan, G.; Sun, J.; Hu, J.; Huang, Y.; Sun, R.; Xu, J.B.; Wong, C.P. Interfacial engineering of silicon carbide nanowire/cellulose microcrystal paper toward high thermal conductivity. ACS Appl. Mater. Interfaces 2016, 8, 31248-31255. [CrossRef] [PubMed]

29. Zhou, Y.; Wang, L.; Zhang, H.; Bai, Y.; Niu, Y.; Wang, H. Enhanced high thermal conductivity and low permittivity of polyimide based composites by core-shell Ag@SiO ${ }_{2}$ nanoparticle fillers. Appl. Phys. Lett. 2012, 101, 012903. [CrossRef]

30. Peters, J.E.; Papavassiliou, D.V.; Grady, B.P. Unique thermal conductivity behavior of single-walled carbon nanotube-polystyrene composites. Macromolecules 2008, 41, 7274-7277. [CrossRef]

31. James, A.C.; John, C.C. Procedures for preparation of silanols. J. Organomet. Chem. 1994, 480, $23-26$.

32. Kim, Y.H.; Bae, J.Y.; Jin, J.; Bae, B.S. Sol-gel derived transparent zirconium-phenyl siloxane hybrid for robust high refractive index LED encapsulant. ACS Appl. Mater. Interfaces 2014, 6, 3115-3121. [CrossRef] [PubMed]

33. Chen, $\mathrm{S}$. Molecular composites comprising $\mathrm{TiO}_{2}$ and their optical properties. Macromolecules 2008, 41, 4838-4844.

(C) 2018 by the authors. Licensee MDPI, Basel, Switzerland. This article is an open access article distributed under the terms and conditions of the Creative Commons Attribution (CC BY) license (http:/ / creativecommons.org/licenses/by/4.0/). 
Article

\title{
A New Route for Preparation of Hydrophobic Silica Nanoparticles Using a Mixture of Poly(dimethylsiloxane) and Diethyl Carbonate
}

\author{
Iryna Protsak ${ }^{1,2,3}$, Evgeniy Pakhlov ${ }^{2}$, Valentyn Tertykh ${ }^{2}$, Zi-Chun Le ${ }^{1, *}$ and Wen Dong ${ }^{3}$ \\ 1 College of Science, Zhejiang University of Technology, Hangzhou 310023, China; ips@zjut.edu.cn \\ 2 Chuiko Institute of Surface Chemistry of National Academy of Sciences of Ukraine, 03164 Kyiv, Ukraine; \\ pakhlov.e.m@gmail.com (E.P.); tertykh@voliacable.com (V.T.) \\ 3 College of Environment, Zhejiang University of Technology, Hangzhou 310023, China; \\ dongwen@zjut.edu.cn \\ * Correspondence: lzc@zjut.edu.cn; Tel.: +86-571-8529-0552
}

Received: 18 January 2018; Accepted: 24 January 2018; Published: 26 January 2018

\begin{abstract}
Organosilicon layers chemically anchored on silica surfaces show high carbon content, good thermal and chemical stability and find numerous applications as fillers in polymer systems, thickeners in dispersing media, and as the stationary phases and carriers in chromatography. Methyl-terminated poly(dimethylsiloxanes) (PDMSs) are typically considered to be inert and not suitable for surface modification because of the absence of readily hydrolyzable groups. Therefore, in this paper, we report a new approach for surface modification of silica $\left(\mathrm{SiO}_{2}\right)$ nanoparticles with poly(dimethylsiloxanes) with different lengths of polymer chains (PDMS-20, PDMS-50, PDMS-100) in the presence of diethyl carbonate (DEC) as initiator of siloxane bond splitting. Infrared spectroscopy (IR), elemental analysis (CHN), transmission electron microscopy (TEM), atomic force microscopy (AFM), rotational viscosity and contact angle of wetting were employed for the characterization of the raw fumed silica and modified silica nanoparticles. Elemental analysis data revealed that the carbon content in the grafted layer is higher than $8 \mathrm{wt} \%$ for all modified silicas, but it decreases significantly after sample treatment in polar media for silicas which were modified using neat PDMS. The IR spectroscopy data indicated full involvement of free silanol groups in the chemisorption process at a relatively low temperature $\left(220^{\circ} \mathrm{C}\right)$ for all resulting samples. The contact angle studies confirmed hydrophobic surface properties of the obtained materials. The rheology results illustrated that fumed silica modified with mixtures of PDMS-x/DEC exhibited thixotropic behavior in industrial oil (I-40A), and exhibited a fully reversible nanostructure and shorter structure recovery time than nanosilicas modified with neat PDMS. The obtained results from AFM and TEM analysis revealed that the modification of fumed silica with mixtures of PDMS-20/DEC allows obtaining narrow particle size distribution with uniform dispersity and an average particle size of $15-17 \mathrm{~nm}$. The fumed silica nanoparticles chemically modified with mixtures of PDMS-x/DEC have potential applications such as nanofillers of various polymeric systems, thickeners in dispersing media, and additives in coatings.
\end{abstract}

Keywords: poly(dimethylsiloxanes); surface modification; nanosilica; diethyl carbonate; carbon content; morphology; coatings; fillers

\section{Introduction}

The hydrophobic nanodispersed silicas are widely used as thickeners in complex polar liquids based on epoxy, polyurethane, and vinylester resins. Additionally, such silicas improve the water resistance of moisture-sensitive formulations such as cosmetic preparations and flowability of powders. Also, chemically modified silicas are widely used as an anti-settling and anti-sagging agent of pigments, 
and in epoxy coatings, respectively. Organophilization of silica surfaces can be performed using various traditional kinds of modifying agents such as alkoxy-, halo-, aminosilanes and organosilazanes [1-8]. However, due to high reactivity and moisture sensitivity of above-mentioned modifying agents, purification is often critical for these hydrolyzable precursors. Poly(dimethylsiloxanes) provide a viable and environmentally benign alternative to the chemical functionalization of oxides as they are characterized by high carbon content, hydrophobic properties, thermal stability, chemical inertness, and they are noncorrosive reagents, and generate only water as a byproduct [9-21]. Despite this fact, the chemisorption of the simplest methylsiloxanes-hexamethyldisiloxane $\left(\mathrm{H}_{3} \mathrm{C}\right)_{3} \mathrm{SiOSi}\left(\mathrm{CH}_{3}\right)_{3}$ on fumed silica surfaces requires the use of very high temperatures (>350 $\left.{ }^{\circ} \mathrm{C}\right)[19,22-24]$. Additionally, the use of poly(dimethylsiloxanes), with high molecular weight for chemical modification of silica surfaces requires an even higher expenditure of energy $\left(\geq 400{ }^{\circ} \mathrm{C}\right)$. One of the probable ways to increase the reactivity of an organosilicon polymer is partial depolymerization of high molecular poly(dimethylsiloxanes) followed by grafting formed oligomers (with terminated alkoxy groups) on silica surfaces. Partial depolymerization can be realized by different means: by thermal degradation (300-400 ${ }^{\circ} \mathrm{C}$ ) or by treatment with toxic agents such as alkalis, sulfuric acids, thionyl chloride, amines, and mixtures of alkali ( $\mathrm{NaOH}, \mathrm{KOH}$ ) with alcohols (methanol, ethanol) [25-30]. It should be noted, however, that the use of these catalysts is technologically complicated because of potential electrolyte presence in the obtained products, which are not desirable especially when using modified silicas as fillers in silicone cable rubbers and thickeners in insulating electrical greases.

In our opinion, new, more favorable conditions come up when using poly(dimethylsiloxanes) for the chemical modification of silica surface in the presence of dialkyl carbonates (DMC or DEC) as initiators of siloxane bond splitting. Dialkyl carbonates are environmentally friendly reagents that meet all the requirements of green chemistry [31,32]. Japanese researchers showed that dimethyl or diethyl carbonate methanol solutions with the addition of alkali metal halogenides are effective agents in reactions of siloxane bond cleavage in the poly(dimethylsiloxanes) [33,34]. For the results of such reactions, alkoxysilanes (dimethyldimethoxysilane and trimethylmethoxysilane, see Scheme 1) were formed [34]:

$$
\mathrm{Me}_{3} \mathrm{Si}-\left[\mathrm{OSiMe}_{2}\right]_{n}-\mathrm{Me}+n(\mathrm{MeO})_{2} \mathrm{CO} \rightarrow(n-1) \mathrm{Me}_{2} \mathrm{Si}(\mathrm{OMe})_{2}+2 \mathrm{Me}_{3} \mathrm{SiOMe}+n \mathrm{CO}_{2}
$$

A recent theoretical study [35] describes the role of the DMC in the Si-O bond cleavage in PDMSs as an activator of $\mathrm{Si}-\mathrm{O}$ bond by coordination $\left[\mathrm{O}=\mathrm{C} \cdots \mathrm{O}-\mathrm{Si}\right.$ and $\left.\mathrm{H}_{3} \mathrm{CO} \cdots \mathrm{Si}-\mathrm{O}\right]$, which largely decreases the electron density at the silicon center, promoting it easer cleavage.

In previous work [36], we determined that DMC without any addition of toxic reagents is an effective agent (initiator) in reactions of siloxane bond splitting in poly(dimethylsiloxanes) and on the silica surface sites. The chemisorption of depolymerized siloxane oligomers on silica surfaces occurred at a relatively low temperature $\left(220^{\circ} \mathrm{C}\right)$. Apart from the obvious technological advantages associated with the use of lower temperatures, this approach allowed us to obtain a high concentration of grafted organic groups in modified products (more than $10 \mathrm{wt} \%$ ). Therefore, it is of interest to examine the effect of diethyl carbonate as initiator of siloxane bond cleavage in organosiloxanes and on surface silica sites when modifying the surface of $\mathrm{SiO}_{2}$ nanoparticles with the purpose to obtain hydrophobic products with uniform dispersity and high carbon content.

Therefore, the aim of the present study was the investigation of the features of DEC chemisorption on $\mathrm{SiO}_{2}$ surface and the chemisorption peculiarities of poly(dimethylsiloxanes) in the presence of DEC on silica surface. The main focus in this paper is lies on (1) the investigation of the reaction conditions for the covalent functionalization of fumed silicas using mixtures of PDMS-x/DEC, (2) the investigation of hydrophobicity of the obtained modified products and the study of their morphology and particle size (3) examining the influence of DEC presence in the modifier mixture on rheological behavior of modified silicas in industrial oil. All samples modified with mixtures of PDMS-x/DEC were compared with hydrophobic silicas modified with neat PDMS. 


\section{Materials and Methods}

\subsection{The Strategy of Silica Surface Modification}

The strategy of the modification process of $\mathrm{SiO}_{2}$ nanoparticles with neat PDMS and PDMS-x/DEC mixtures (Scheme 1) was adopted from our previous work [36]. The chemisorption of PDMS on the fumed silica surface was performed at $220^{\circ} \mathrm{C}$ for $2 \mathrm{~h}$ with or without addition of DEC (the volume ratio of PDMS-x/DEC was 1:1). Overall, the PDMS amount was $17 \%$ of silica weight. The modification of silica was carried out in a glass reactor with a glass stirrer (at rotational speed from 20 to $300 \mathrm{rpm}$ ). After loading with fumed silica, all the air volume in the reactor was replaced with nitrogen and the reactor was heated up to $220^{\circ} \mathrm{C}$. Further, the reactor filled with nitrogen was stopped and the modifying agent was added by spraying its aerosol through the nozzle. After heating, the mixture was cooled to room temperature. The removal of the physically adsorbed reactants was performed in a Soxhlet apparatus with $n$-hexane as a solvent at $68^{\circ} \mathrm{C}$ for $1 \mathrm{~h}$. Then, the washed samples were dried in the muffle furnace (ThermoLab SNOL 7,2/1100, Kyiv, Ukraine) at $80^{\circ} \mathrm{C}$ for $2 \mathrm{~h}$.

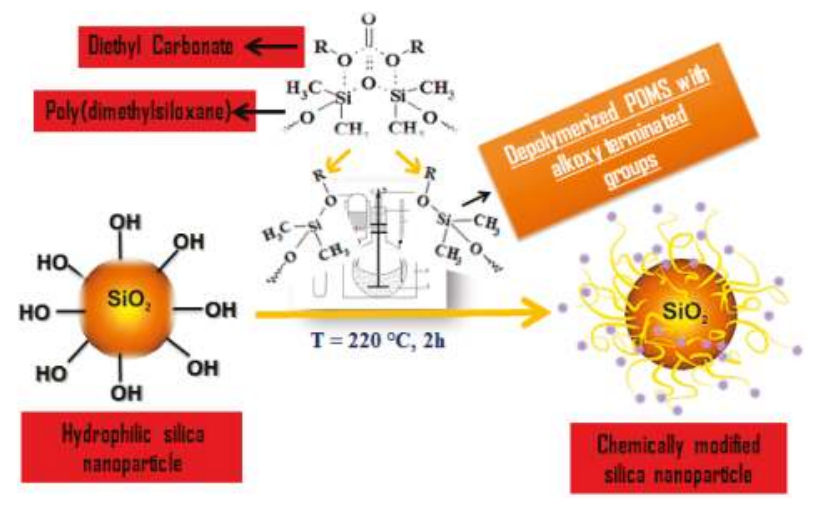

Scheme 1. Synthesis of high disperse silicas with a high content of the grafted organic groups.

\subsection{Reagents}

Fumed silica (purity 99.87\%, S=260 $\mathrm{m}^{2} / \mathrm{g}$ ) were supplied by the Pilot Plant of the Chuiko Institute of Surface Chemistry (Kalush, Ukraine). Commercial poly(dimethylsiloxanes) (code name: PDMS-100, linear, $-\mathrm{CH}_{3}$ terminated, viscosity $95-105 \mathrm{~mm}^{2} / \mathrm{s}$ molecular weight $\sim 6000 \mathrm{Da}$ and degree of polymerization 35-65), (code name: PDMS-50, linear, $-\mathrm{CH}_{3}$ terminated, viscosity $45-55 \mathrm{~mm}^{2} / \mathrm{s}$, molecular weight $\sim 3500 \mathrm{Da}$ and degree of polymerization 23-28) and poly(dimethylsiloxane) (code name: PDMS-20, linear, $-\mathrm{CH}_{3}$ terminated, viscosity $18-22 \mathrm{~mm}^{2} / \mathrm{s}$, molecular weight $2000 \mathrm{Da}$ and degree of polymerization 13-15). Diethyl carbonate containing $\geq 99.0 \mathrm{wt} \%$ of $\left(\mathrm{C}_{2} \mathrm{H}_{5} \mathrm{O}\right)_{2} \mathrm{CO}$, $n$-hexane containing $\geq 99.0 \mathrm{wt} \%$ of $\mathrm{C}_{6} \mathrm{H}_{6}$ and hexamethyldisilazane (HMDS) was obtained by Sigma Aldrich (Hamburg, Germany).

\subsection{Infrared Spectroscopy}

In order to control the flow of surface reactions, IR spectra were recorded using a Specord M-80 spectrophotometer (Carl Zeiss, Jena, Germany) in the $4000-200 \mathrm{~cm}^{-1}$ wavenumber range. The silica samples were pressed into rectangular $28 \times 8 \mathrm{~mm}$ plates of $25 \mathrm{mg}$ weight.

The spectral investigations of the chemisorption processes of DEC on the silica surface were conducted in the quartz cuvette, which allowed us to perform thermal processing of the samples in vacuum conditions. The quartz cuvette contained infrared transparent glass made of fluorite. In the first stage, the sample of fumed silica was heated at $600{ }^{\circ} \mathrm{C}$ and pumped at a pressure of $10^{-2} \mathrm{~mm} \cdot \mathrm{Hg}$ 
in order to remove the interfacial and adsorbed water from the silica surface. At the second stage, the degassing of diethyl carbonate was performed by means of the freeze-pump-thaw degassing method. At the third stage, the prepared sample of dehydrated silica was brought into contact with saturated vapors of diethyl carbonate at different temperatures $\left(200,300\right.$ and $\left.350{ }^{\circ} \mathrm{C}\right)$.

\subsection{Contact Angles}

Contact angle measurements were performed using a commercial Contact Angle Meter (GBX Scientific Instruments, Romans sur Isere, France) equipped with a temperature and humidity controlled measuring chamber and a digital camera $\left(20{ }^{\circ} \mathrm{C}\right.$, relatively humidity is $\left.50 \%\right)$. Firstly, hydrophobic powders were pressed into thin pellets (180 bars for $15 \mathrm{~min}$ ). Then, a drop of deionized water was placed onto the surface and then plate was checked to determine the distribution of water on the surface. The contact angle was calculated using a computer program from a measurement of the width and height of the droplet. To obtain the averaged values, the measurements were performed for 6 water droplets put on each sample.

\subsection{Elemental Analysis}

The content of grafted organic groups in the synthesized samples was measured by Perkin-Elmer $2400 \mathrm{CHN}$-analyzer (Waltham, MA, USA). The anchored layer was oxidized to produce $\mathrm{H}_{2} \mathrm{O}$ and $\mathrm{CO}_{2}$ during the samples heating in the oxygen flow at $750{ }^{\circ} \mathrm{C}$.

\subsection{TEM and AFM}

Transmission electron microscopy (TEM) images were recorded using FEI Tecnai G2 T20 X-TWIN, Hillssboro, OR, USA. The powder samples were added to acetone (chromatographic grade) and sonicated. Then, a drop of the suspension was deposited onto a copper grid with a thin carbon film. After acetone evaporation, sample particles that remained on the film were studied with TEM. Additionally, the surface morphology and grain size distribution was analyzed using atomic force microscopy (AFM, Nanoscope V Digital Instruments, Boston, MA, USA, with a Tapping Mode technique). AFM data processing was performed using the SPIP program (version 5.0.6, Hørsholm, Denmark).

\subsection{Rheology of Modified Silica Nanoparticles}

Modified silica suspensions were prepared at concentrations of solid phase $5 \mathrm{wt} \%$ in industrial oil (I-40A) at room temperature $\left(25^{\circ} \mathrm{C}\right)$. Rheological properties of the silica suspensions were studied with a rotational viscosimeter Reotest RV2.1 ("Mettingen" firm, Mettingen, Germany,) equipped with a cylindrical system at the shear rates $(\gamma)$ from 9 to $1312.2 \mathrm{~s}^{-1}$. The industrial oil was employed as a dispersion media as hydrophobic silica has a wide range of applications as thickeners for nonpolar fluids.

\section{Results and Discussion}

\subsection{Infrared Spectroscopy Analysis Results}

We have already shown [36] that chemical interaction of dimethyl carbonate with sites of the dehydrated silica surface takes place at a temperature of $200{ }^{\circ} \mathrm{C}$ and higher. Chemisorption processes involve both structural silanol groups and siloxane bridges on the surface. In this paper, we investigated the peculiarities of diethyl carbonate chemisorption on the dehydrated silica surface. At the first stage, both features of the interaction of diethyl carbonate with structural silanol groups on the silica surface (see Scheme 2I) and possibilities of the cleavage of siloxane bonds located directly on the dehydrated silica surface were investigated (see Scheme 2II). 


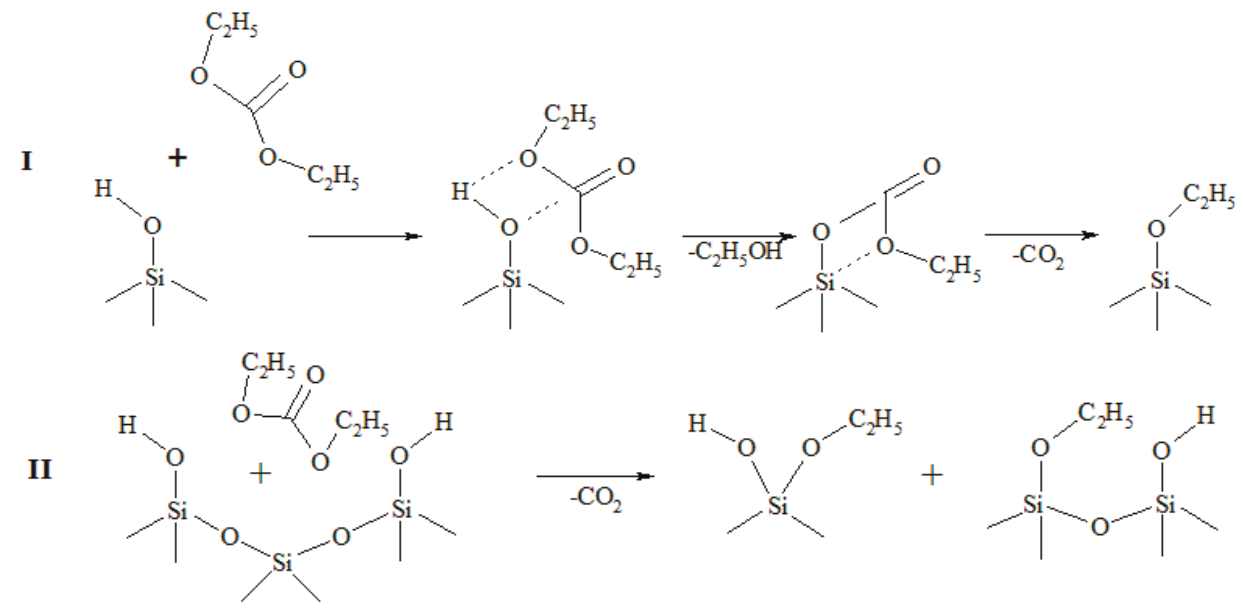

Scheme 2. (I) Attack by diethyl carbonate (DEC) of silica silanol group; (II) attack by DEC of silica $\mathrm{Si}-\mathrm{O}$ bond.

It was found that the chemical interaction of DEC $\left(\left(\mathrm{H}_{5} \mathrm{C}_{2} \mathrm{O}\right)_{2} \mathrm{CO}\right)$ with the surface sites of dehydrated silica takes place when the temperature increases up to $200{ }^{\circ} \mathrm{C}$ (Figure 1a).

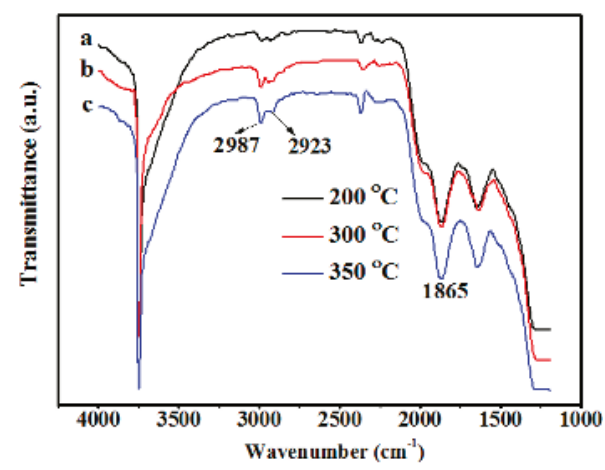

Figure 1. IR spectra of dehydrated silica after interaction with saturated vapors of diethyl carbonate at (a) $200{ }^{\circ} \mathrm{C}$; (b) $300{ }^{\circ} \mathrm{C}$; (c) $350{ }^{\circ} \mathrm{C}$ and subsequent vacuum treatment of the samples at the same temperatures.

When the temperature rises further, the increase in the intensity of bands corresponding to the stretching vibrations C-H at 2987 and $2923 \mathrm{~cm}^{-1}$ in grafted ethoxy groups are observed. It should be noted that the concentration of grafted ethoxy groups $\left(-\mathrm{OC}_{2} \mathrm{H}_{5}\right)$ increases and full participation of silanol groups $(\mathrm{O}-\mathrm{H})$ in the chemical reaction with diethyl carbonate under these conditions is not observed. Therefore, it was logical to assume that the chemisorption of $\left(\mathrm{H}_{5} \mathrm{C}_{2} \mathrm{O}\right)_{2} \mathrm{CO}$ proceeds via the siloxane bond cleavage on the silica surface. To test this assumption and to better understand the processes of chemisorption of diethyl carbonate, the silanol groups were removed from the silica surface by being substituted with trimethylsilyl groups as a result of the reaction with hexamethyldisilazane (Figure 2b). 


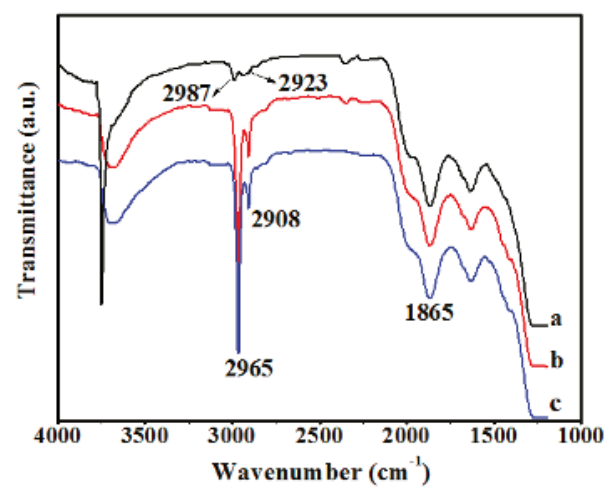

Figure 2. IR spectra of fumed silica after (a) reaction with vapors of diethyl carbonate; (b) reaction with vapors of hexamethyldisilazane at $120{ }^{\circ} \mathrm{C}$ subsequent vacuum treatment of surface at $300{ }^{\circ} \mathrm{C}$; (c) interaction with vapors of the diethyl carbonate and vacuum treatment at $300{ }^{\circ} \mathrm{C}$.

From Figure $2 b$, we can see that silanol groups are not observed on the spectra with simultaneous appearance of the stretching vibrations of methyl groups $\left(2908-2965 \mathrm{~cm}^{-1}\right)$. However, after the contact with vapors of $\left(\mathrm{H}_{5} \mathrm{C}_{2} \mathrm{O}\right)_{2} \mathrm{CO}$ at $300{ }^{\circ} \mathrm{C}$ and the subsequent vacuum treatment of the surface, the band with maximum at 2923 and $2987 \mathrm{~cm}^{-1}$ is very hard to distinguish from the valence vibrations of methyl groups as they absorb at the same frequency ranges (Figure 2c, see Scheme 3).

Stretching vibrations of methyl groups at $2965-2908 \mathrm{~cm}^{-1}$ Stretching vibrations of ethoxy groups at $2987-2923 \mathrm{~cm}^{-1}$<smiles>CCO[Si](C)(C)O[Si](C)(C)O[Si](C)(C)C(=O)[O-]</smiles>

Scheme 3. Scheme of interaction of DEC with silica surface which were treated with HMDS at $300{ }^{\circ} \mathrm{C}$.

In summary, we can say that DEC reacts with silanol groups on the silica surface forming the grafted ethoxy groups which can act as an additional reactive center for chemisorption of organosiloxanes. However, it is very hard to say that DEC reacts with the $\mathrm{Si}-\mathrm{O}$ bond directly at the $\mathrm{SiO}_{2}$ surface, but we can assume that this reaction may occur due to the above-mentioned context.

Changes in surface structure of modified silicas are clearly visible in the IR spectra (Figure 3). Figure 3 shows the IR spectra of neat $\mathrm{SiO}_{2}$ nanoparticles (Figure 3a), silica modified with neat DEC (Figure $3 \mathrm{~b}$ ) and composites prepared by modification of $\mathrm{SiO}_{2}$ surface with neat poly(dimethylsiloxanes) (PDMS-20 or PDMS-50) and their mixture with DEC (Figure 3c-f). In the spectrum of the silica which was modified only with diethyl carbonate (Figure 3b), we can see the presence of valence vibrations of $\mathrm{C}-\mathrm{H}$ bond in ethoxy groups at $2987-2908 \mathrm{~cm}^{-1}$, the peak attributed to absorbed water at $3700-3400 \mathrm{~cm}^{-1}$ and $1620 \mathrm{~cm}^{-1}$ and also vibration of silanol groups at $3750 \mathrm{~cm}^{-1}$. The spectrum of neat fumed silica (Figure 3a) is characterized by the presence of valence vibration of silanol groups at $3750 \mathrm{~cm}^{-1}$ and the valence and deformation vibration of absorbed water at $3700-3400 \mathrm{~cm}^{-1}$ and $1620 \mathrm{~cm}^{-1}$, respectively. Intensive band at $2965 \mathrm{~cm}^{-1}$ (asymmetric C-H vibrations in methyl group) 
and accompanying band at $2908 \mathrm{~cm}^{-1}$ (symmetric C-H vibrations) in IR spectra of modified silicas samples indicate high concentration of grafted methylsiloxane which is in accordance with the data on carbon content (Figure 4, discussed in Section 3.2) demonstrating high yield of methylsiloxane grafting.

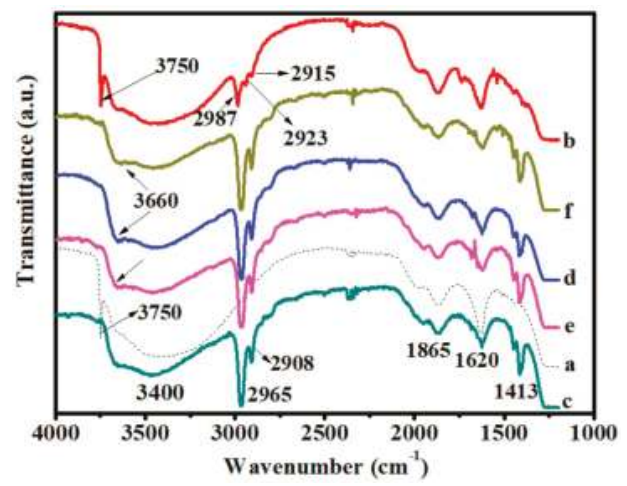

Figure 3. IR spectra of (a) neat fumed silica; (b) fumed silica modified with neat DEC; and (c) silica modified with neat poly(dimethylsiloxanes) (PDMS)-50; (d) PDMS-20 and ((e,f) respectively) their mixtures with DEC at $220^{\circ} \mathrm{C}$ for $2 \mathrm{~h}$.

However, the free silanol band at $3750 \mathrm{~cm}^{-1}$ is no longer detected. The signal of the free silanol groups disappeared completely from the spectra of modified silicas confirms the passage of the reaction between the silica surface and modifier agents. Note that the intensity of a silanol band at $3660 \mathrm{~cm}^{-1}$ changes much less than the $3750 \mathrm{~cm}^{-1}$ free silanol band. The former is attributed to silanols that are less accessible [37] to siloxane molecules during the surface modification. In addition, we can see that the intensity of band at $3700-3400$ and $1620 \mathrm{~cm}^{-1}$ corresponding to adsorbed water is detectable on the surface of all modified samples, but the intensity of this peak slightly decreases for silicas modified by mixtures of PDMS-x/DEC (Figure 3e,f). This could be explained by that fact that siloxane oligomers which were formed as the result of DEC and PDMS interaction have reacted with surface silanols more intensively than neat PDMS macromolecules.

At any rate, we still can see that individual molecules or clusters of water remained in the adsorption layer of oxide composites despite the presence of hydrophobic PDMS. The presence of adsorbed water clusters can be explained by the textural features of the nanosilica powders. Water molecules are much smaller than the cross-section of PDMS. Therefore, water can penetrate into narrow nanovoids in the contact zones between adjacent nanoparticles in aggregates but PDMS molecules cannot penetrate into these voids.

\subsection{Elemental Analysis Results}

From the elemental analysis data (Figure 4), one can see that the highest concentration of grafted organic groups in modified samples ( $>9 \mathrm{wt} \%$ of carbon content) is achieved by using of PDMS-50/DEC mixture for the modification.

In addition, we can see that the carbon content is not practically changed after the treatment in organic solution for silicas modified with mixtures of PDMS-x/DEC, which indicates that organosiloxanes are chemically bonded with the silica surface sites. For samples modified in the absence of DEC after the treatment in the same conditions, the carbon content decreases about $3 \mathrm{wt} \%$, which is evidence of partial desorption of the grafted organic layer into the solvent. The obtained elemental analysis data is in good agreement with data of rheology, discussed later. 


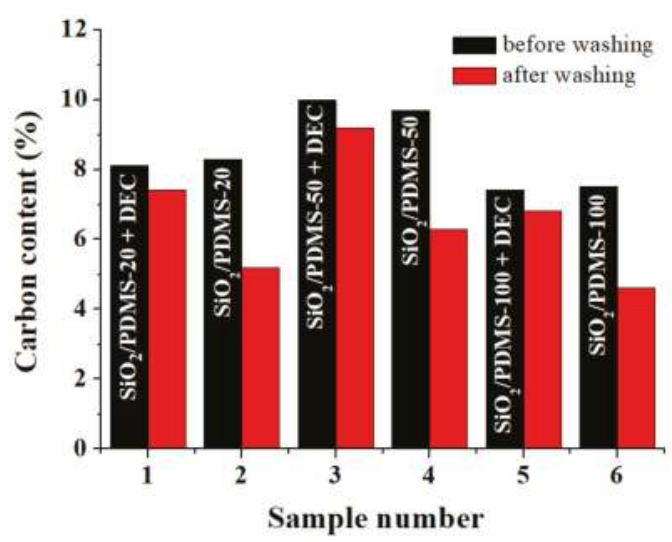

Figure 4. Histogram of carbon content for modified silicas: 1- $\mathrm{SiO}_{2} / \mathrm{PDMS}-20+\mathrm{DEC}$, 2- $\mathrm{SiO}_{2} / \mathrm{PDMS}-20,3-\mathrm{SiO}_{2} / \mathrm{PDMS}-50+\mathrm{DEC}, 4-\mathrm{SiO}_{2} / \mathrm{PDMS}-50,5-\mathrm{SiO}_{2} / \mathrm{PDMS}-100+\mathrm{DEC}$, 6- $\mathrm{SiO}_{2} / \mathrm{PDMS}-100$.

The value of the contact angle of wetting (Figure 5) for such powders was measured to be about $115-116^{\circ}$ which indicates the hydrophobic surface. Samples synthesized in the presence of DEC were characterized by slightly higher values of the contact angle of wetting.

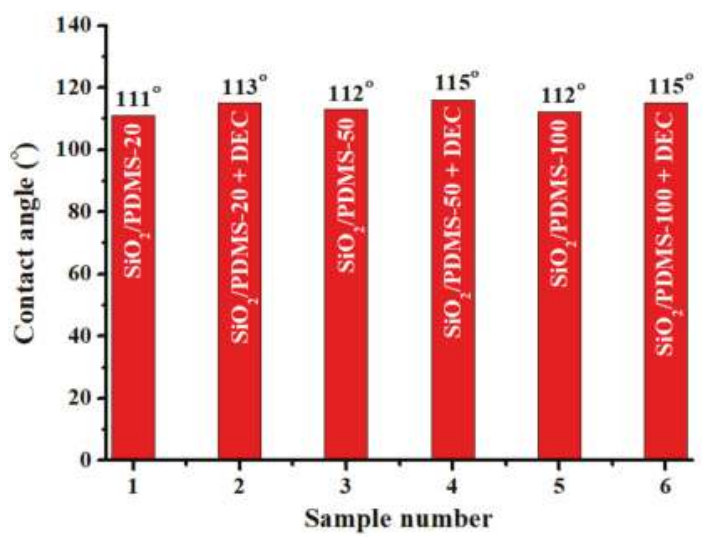

Figure 5. Contact angles of wetting of modified silicas.

\subsection{Rheology Analysis Results}

Modified disperse silicas are widely used as rheological additives, which are key ingredients in paints, coatings and inks as they control the precise properties and characteristics of fluid products. Therefore, the rheological properties in industrial oil of modified silicas were examined (Figure 6a-d).

Due to electrostatic interactions, silicas modified with neat organosiloxanes (PDMS-20 or PDMS-50) and their mixture of DEC disperse in coating formulations and create a three-dimensional network, which results in a viscosity increase in the formulation. Under shear force, the three-dimensional network collapses and agglomerates of modified silica move freely through the liquid, and as a result viscosity decreases (Figure 6a-d). After the shear force weakens, the modified silica network spontaneously reforms and the viscosity increases again (Figure 6a-d). This fully reversible network is the key to success for rheology control with modified silicas. Figure $6 \mathrm{~b}$, d reveals 
that dispersion of modified silicas with neat PDMS-20 or PDMS-50 are characterized by a low structure recovery of the interparticle bonds by reducing a shear rate, i.e., with mechanical influence on the thickened suspensions a partial destruction of the structure is observed. In contrast, silica dispersions modified with mixtures of PDMS-20 or PDMS-50 in the presence of DEC (Figure 6a,c) are clearly characterized by a fully reversible inner structure and thixotropic behavior as the viscosity increases to initial values. In addition to this, silicas modified with mixtures of PDMS-x/DEC require shorter time for the structure recovery after the shear force was applied which can be explained by the formation of stable bonds between silica and organosilicone oligomer. On the contrary, for $\mathrm{SiO}_{2}$ which was modified with neat PDMS-20 or PDMS-50 the definite part of oligomer is physically adsorbed on the surface and a partial destruction of the colloidal structure is observed under the mechanical action as the viscosity does not increases to initial values.

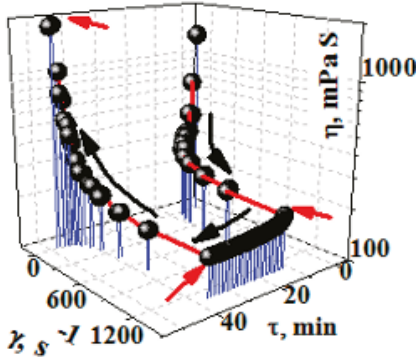

(a)

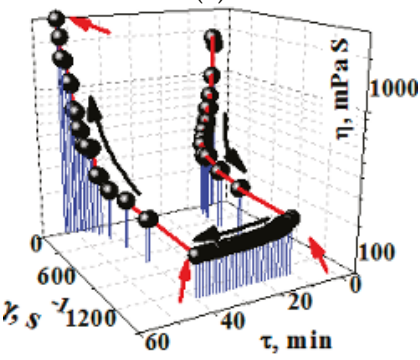

(c)

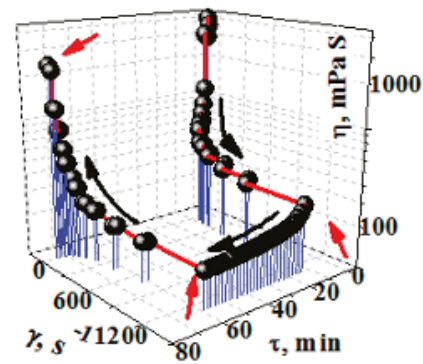

(b)

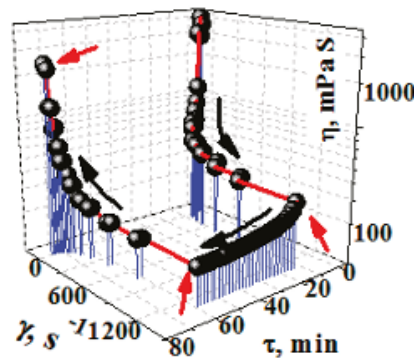

(d)

Figure 6. Dependence of effective viscosity on the shear rate over time for hydrophobic silica modified with (a) mixture of PDMS-20/DEC; (b) neat PDMS-20; (c) mixture of PDMS-50/DEC; (d) neat PDMS-50 in industrial oil I-40A with the concentration of solid phase $5 \mathrm{wt} \%$.

\subsection{AFM and TEM Analysis Results}

Figure 7 illustrates AFM images and the histogram (depicting the nanoparticle size distribution) of initial $\mathrm{SiO}_{2}$ nanoparticles and fumed silica modified with neat PDMS-20 and its mixture with DEC.

Notice that particles of modified silicas are smaller (Figure $7 \mathrm{~b}-\mathrm{d}$ ) than those of bare $\mathrm{SiO}_{2}$ (Figure $7 \mathrm{a}, \mathrm{d}$ ). This occurs due to the heating of modified samples during synthesis and thus the formation of smaller nanoparticles. The silica modified with mixture of PDMS-20/DEC (Figure 7c,d) characterized by homogenous particle size distribution has an average grain size of $15-17 \mathrm{~nm}$. This is easy to explain assuming that firstly, DEC can act as a blocker and a separator that prevents the aggregation of modified $\mathrm{SiO}_{2}$ nanoparticles and thus hinders any further growth of $\mathrm{SiO}_{2}$ particles; secondly, the chemisorption of depolymerized poly(dimethylsiloxane) accompanied by the grafting of shorter polymer chains on the $\mathrm{SiO}_{2}$ surface which improves the distribution of the depolymerized PDMS-20 on the $\mathrm{SiO}_{2}$ surface; thirdly, the short polymeric grafted chains can control the nanoparticle aggregation by steric repulsions. 


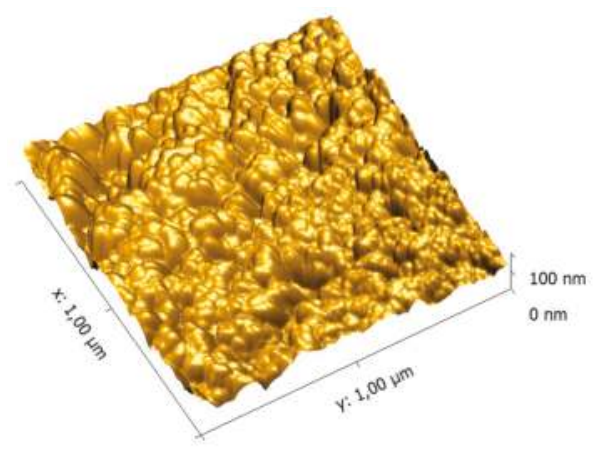

(a)

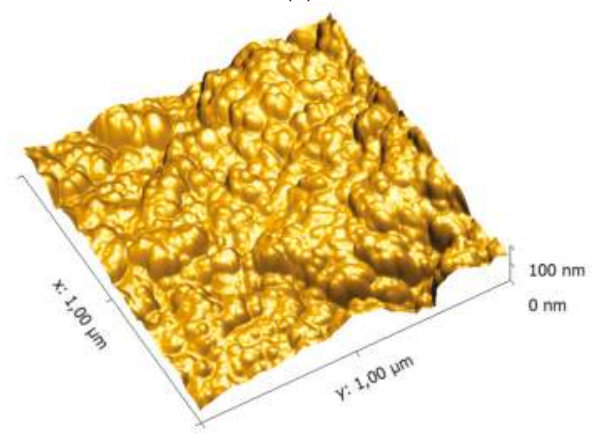

(c)

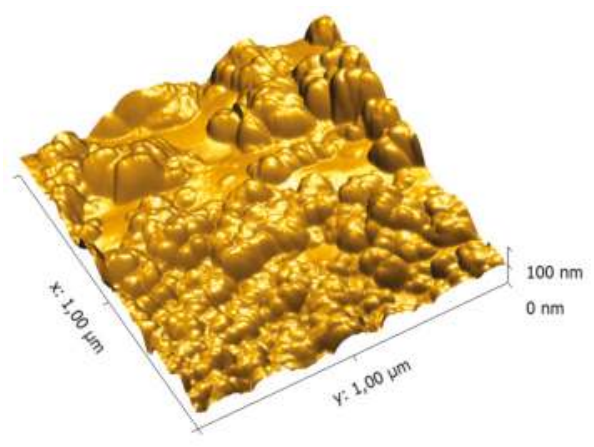

(b)

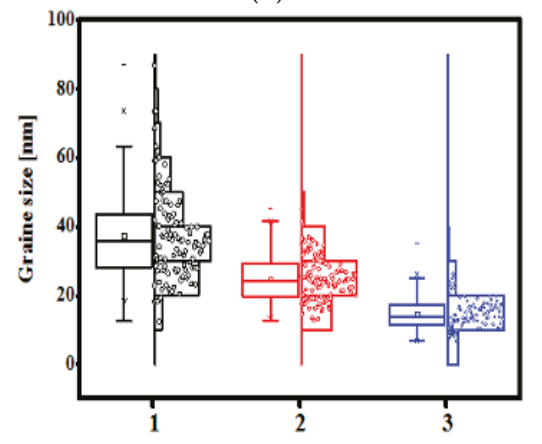

(d)

Figure 7. Atomic force microscopy (AFM) images of (a) initial $\mathrm{SiO}_{2}$ nanoparticles; (b) $\mathrm{SiO}_{2}$ modified with neat PDMS-20; (c) $\mathrm{SiO}_{2}$ modified with mixtures of PDMS-20/DEC and (d) their histogram: 1 - initial $\mathrm{SiO}_{2}$ nanoparticles, $2-\mathrm{SiO}_{2}$ modified with neat PDMS-20, 3- $\mathrm{SiO}_{2}$ modified with mixtures of PDMS-20/DEC.

In contrast, the modification of $\mathrm{SiO}_{2}$ with neat PDMS-20 (Figure 7b,d) leads to an increase in particle size up to $25-30 \mathrm{~nm}$. This can be explained by the fact that the reaction of neat polymers with $\mathrm{SiO}_{2}$ surface can run through the island-like polymer distribution onto the silica surface (as the chains of neat polymers is very long and it is difficult to spread them out over the surface) when bonded molecules favor other molecules to be bound nearby which may cause the aggregation of $\mathrm{SiO}_{2}$ nanoparticles.

The morphology of neat silica and modified silicas were also analyzed using transmission electron microscopy (Figure 8). Initial fumed silica in this study is composed of nonporous nanoparticles with a true density of amorphous nanosilica $\rho_{\mathrm{O}}=2.2 \mathrm{~g} / \mathrm{cm}^{3}$ forming aggregates (Figure $8 \mathrm{a}$ ) and agglomerates of aggregates. The particles obtained using for the modification of $\mathrm{SiO}_{2}$ mixtures of PDMS-20/DEC exhibit a lesser degree of aggregation than those obtained via modification of $\mathrm{SiO}_{2}$ surfaces by neat PDMS-20. $\mathrm{SiO}_{2}$, grafted with depolymerized PDMS-20 shows no sign of a visible coating (Figure 8b). Therefore, it is assumed that the functionalization took place via the attachment of a monolayer of the respective PDMS-20/DEC mixture on the surface of $\mathrm{SiO}_{2}$. On the contrary, it is possible to depict some part of polymer on $\mathrm{SiO}_{2}$ surface which was modified with neat PDMS-20 (Figure 8c). Voids between primary nanoparticles in the secondary structures are responsible for the textural porosity of the silica powders and they are present for both bare silica and for modified samples [37-41]. In general, the hydrophobization of the silica surface with siloxanes to form a mosaic coverage with methyl groups affects the particulate morphology. 


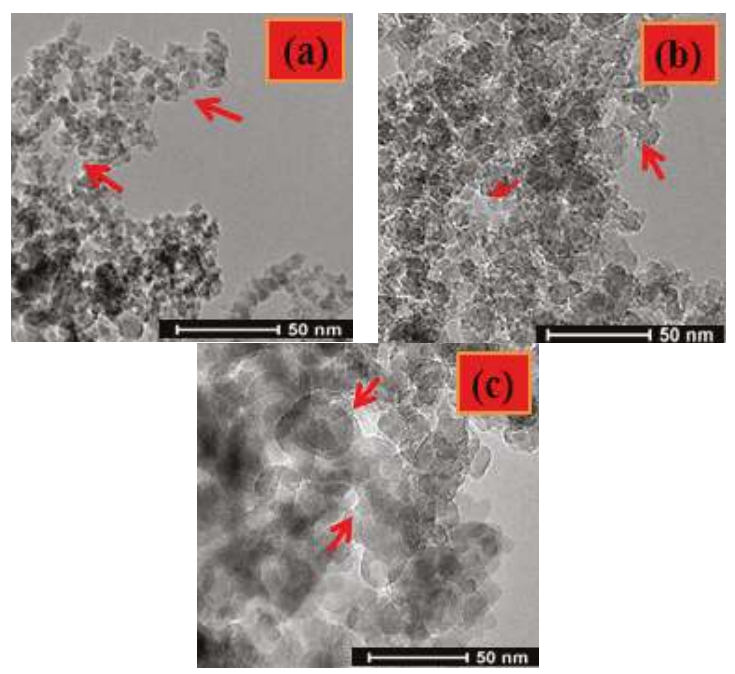

Figure 8. Transmission electron microscopy (TEM) images of (a) bare fumed silica; (b) silicas modified with mixtures of PDMS-20/DEC and (c) neat PDMS-20.

\section{Conclusions}

In the present work, the features of DEC chemisorption on a fumed silica surface were examined, and it was found that the chemical interaction of diethyl carbonate with sites of the dehydrated silica surface takes place at a temperature of $200^{\circ} \mathrm{C}$ and higher, and that chemisorption processes involve structural silanol groups.

The reaction of neat poly(dimethylsiloxanes) and their mixture with DEC with fumed silica were investigated. It was established that the reaction of siloxane oligomers with free silanol groups on silica surface in the presence of diethyl carbonate takes place at relatively low temperatures $\left(220^{\circ} \mathrm{C}\right)$ with the formation of stable hydrophobic coating (exhibiting contact angles $113-115^{\circ}$ ) in which the carbon content is higher than $8 \mathrm{wt} \%$. The modification of $\mathrm{SiO}_{2}$ surface with organosiloxanes without diethyl carbonate leads to the formation of patches of an absorbed organic layer which is easily desorbed in polar media.

The results of TEM and AFM studies revealed that modified $\mathrm{SiO}_{2}$ nanoparticles with mixtures of PDMS-20/DEC exhibit a lesser degree of aggregation than those obtained using neat PDMS-20 for modification. The average particle size of the modified $\mathrm{SiO}_{2}$ with mixtures of PDMS-20/DEC is determined to be $15-17 \mathrm{~nm}$. The research of rheological properties of modified silicas in industrial oil has shown that the dispersion of silicas, which were modified with mixtures of PDMS-x/DEC exhibits thixotropic behavior and is characterized by a fully reversible network and a short recovering time. In contrast, the partially irreversible destruction of the structure is observed for $\mathrm{SiO}_{2}$ modified with neat PDMS.

To summarize, we have developed a highly efficient (carbon content $>8 \mathrm{wt} \%$ ), relatively low temperature $\left(220^{\circ} \mathrm{C}\right)$ and environmentally friendly grafting approach for surface functionalization of silicas by using mixtures of PDMS-x/DEC. Such silicas, with a covalently attached hydrophobic layer, can find their applications as additives in coatings and paints, fillers in various polymeric systems and thickeners in optic cable gels.

Acknowledgments: This research was supported by the People Programme (Marie Curie Actions) of the European Union's Seventh Framework Programme FP7/2007-2013/under grants agreement No 612484, the Special Funding of "The Belt and Road" International Cooperation of Zhejiang Province (2015C04005) and the National Natural Science Foundation of China (61571399). 
Author Contributions: Iryna Protsak, Valentyn Tertykh, and Wen Dong conceived and designed the experiments; Evgeniy Pakhlov performed the IR experiment; Iryna Protsak performed all other experiments; Iryna Protsak analyzed the data; Zi-Chun Le contributed reagents/materials/analysis tools; Iryna Protsak wrote the paper.

Conflicts of Interest: The authors declare no conflict of interest.

\section{References}

1. Litvinov, V.M.; Barthel, H.; Weis, J. Structure of a PDMS Layer Grafted onto a Silica Surface Studied by Means of DSC and Solid-State NMR. Macromolecules 2002, 35, 4356-4364. [CrossRef]

2. Rao, A.V.; Kulkarni, M.; Amalnerkar, D.P.; Seth, T. Surface chemical modification of silica aerogels using various alkyl-alkoxy/chloro silanes. Appl. Surf. Sci. 2003, 206, 262-270. [CrossRef]

3. Park, S.E.; Prasetyanto, E.A. Morphosynthesis and Catalysis by Organofunctionalized Mesoporous Materials. In Organosilanes, Properties, Performance, and Applications, UK ed.; Wyman, E.B., Skief, M.C., Eds.; Nova Science Publishers: New York, NY, USA, 2010; pp. 101-131, ISBN 978-1-60876-452-5.

4. Daoud, W.A.; Xin, J.H.; Xiaoming, T. Synthesis and characterization of hydrophobic silica nanocomposites. Appl. Surf. Sci. 2006, 252, 5368-5371. [CrossRef]

5. Bernardoni, F.; Kouba, M.; Fadeev, A.Y. Effect of curvature on the packing and ordering of organosilane monolayers supported on solids. Chem. Mater. 2008, 20, 382-387. [CrossRef]

6. Moitra, N.; Ichii, S.; Kamei, T.; Kanamori, K.; Zhu, Y.; Takeda, K.; Nakanishi, K.; Shimada, T. Surface Functionalization of Silica by Si-H Activation of Hydrosilanes. J. Am. Chem. Soc. 2014, 136, 11570-11573. [CrossRef] [PubMed]

7. Fadeev, A.Y.; McCarthy, T.J. Self-assembly is not the only reaction possible between alkyltrichlorosilanes and surfaces: Monomolecular and oligomeric covalently attached layers of dichloro- and trichloro alkylsilanes on silicon. Langmuir 2000, 16, 7268-7274. [CrossRef]

8. Fadeev, A.Y.; McCarthy, T.J. Binary monolayer mixtures: Modification of nanopores in silicon-supported tris(trimethylsiloxy)silyl monolayers. Langmuir 1999, 15, 7238-7243. [CrossRef]

9. Li, Y.-F.; Xia, Y.-X.; Xu, D.-P.; Li, G.-L. Surface Reaction of Particulate Silica with Polydimethylsiloxanes. J. Polym. Sci. 1981, 19, 3069-3079. [CrossRef]

10. Fadeev, A.Y.; Kazakevich, Y.V. Covalently attached monolayers of oligo(dimethylsiloxane)s on silica: A siloxane chemistry approach for surface modification. Langmuir 2002, 18, 2665-2672. [CrossRef]

11. Graffius, G.; Bernardoni, F.; Fadeev, A.Y. Covalent Functionalization of Silica Surface Using "Inert" Poly(dimethylsiloxanes). Langmuir 2014, 30, 14797-14807. [CrossRef] [PubMed]

12. Protsak, I.S.; Kuzema, P.O.; Tertykh, V.A.; Bolbukh, Y.M.; Kozakevich, R.B. Thermogravimetric analysis of silicas chemically modified with products of deoligomerization of polydimethylsiloxane. J. Therm. Anal. Calorim. 2015, 121, 547-557. [CrossRef]

13. Guba, G.Y.; Bogillo, V.I.; Chuiko, A.A. Kinetics and mechanism of the reaction of organosiloxanes with the surface of pyrogenic silica. Theor. Exp. Chem. 1993, 28, 146-150. [CrossRef]

14. Xiao, D.; Zhang, H.; Wirth, M. Chemical Modification of the Surface of Poly(dimethylsiloxane) by Atom-Transfer Radical Polymerization of Acrylamide. Langmuir 2002, 18, 9971-9976. [CrossRef]

15. Barthel, H.; Nikitina, E. INS and IR study of Intermolecular Interactions at the Fumed Silica-Polydimethylsiloxane Interphase, Part 3. Silica-Siloxane Adsorption Complexes. Silicon Chem. 2004, 1, 261-279. [CrossRef]

16. Smith, J.S.; Borodin, O.; Smith, G.D.; Kober, E.M. A Molecular Dynamics Simulation and Quantum Chemistry Study of Poly(dimethylsiloxane)-Silica Nanoparticle Interactions. J. Polym. Sci. B 2007, 45, 1599-1615. [CrossRef]

17. Gun'ko, V.M.; Borysenko, M.V.; Pissis, P.; Spanoudaki, A.; Shinyashiki, N.; Sulim, I.Y.; Kulik, T.V.; Palyanytsy, B.B. Polydimethylsiloxane at the interfaces of fumed silica and zirconia/fumed silica. Appl. Surf. Sci. 2007, 253, 7143-7156. [CrossRef]

18. Sulym, I.Y.; Borysenko, M.V.; Goncharuk, O.V.; Terpilowski, K.; Sternik, D.; Chibowski, E.; Gun'ko, V.M. Structural and hydrophobic-hydrophilic properties of nanosilica/zirconia alone and with adsorbed PDMS. Appl. Surf. Sci. 2011, 258, 270-277. [CrossRef]

19. Krumpfer, J.W.; McCarthy, T.J. Rediscovering Silicones: "Unreactive" Silicones React with Inorganic Surfaces. Langmuir 2011, 27, 11514-11519. [CrossRef] [PubMed] 
20. Qiang, Z.; Wadley, M.L.; Vogt, B.D.; Cavicchi, K.A. Facile Non-Lithographic Route to Highly Aligned Silica Nanopatterns Using Unidirectionally Aligned Polystryrene-Block-Polydimethysiloxane Films. J. Polym. Sci. B 2015, 53, 1058-1064. [CrossRef]

21. Palacios-Pineda, L.M.; Perales-Martinez, I.A.; Lozano-Sanchez, L.M.; Martínez-Romero, O.; Puente-Córdova, J.; Segura-Cárdenas, E.; Elías-Zúñiga, A. Experimental Investigation of the Magnetorheological Behavior of PDMS Elastomer Reinforced with Iron Micro/Nanoparticles. Polymers 2017, 9, 696. [CrossRef]

22. Aristova, V.G.; Zimmer, I.M.; Gorbunov, A.I.; Zhilenkov, I.V.; Saushkin, V.V. Mechanism of Siloxane Bond Splitting on a Hydrated Aerosil Surface. Dokl. Akad. Nauk SSSR 1980, 255, 131-134.

23. Tertykh, V.A.; Pavlov, V.V. Reactivity of molecules attacking a functional center. Adsorpt. Adsorbt. Duide 1978, $6,67-75$.

24. Pavlov, V.V.; Guba, G.Y.; Tertykh, V.A.; Chuiko, A.A. Study of the interaction of alkylsiloxanes with the surface of dispersed silicas. Adsorpt. Adsorbt. Duide 1980, 8, 35-39.

25. Chang, C.L.; Lee, H.S.; Chen, C.K. Aminolysis of cured siloxane polymers. Polym. Degrad. Stab. 1999, 65, 1-4. [CrossRef]

26. Thomas, T.H.; Kendrick, T.C. Thermal Analysis of Polydimethylsiloxanes. I. Thermal Degradation in Controlled Atmospheres. J. Polym. Sci. B 1969, 7, 537-549. [CrossRef]

27. Clarson, S.J.; Semlyen, J.A. Studies of Cyclic and Linear Poly(dimethylsiloxanes): 21. High Temperature Thermal Behavior. Polymer 1986, 27, 91-95. [CrossRef]

28. Hsiao, Y.C.; Hill, L.W.; Pappas, S.P. Reversible amine solubilization of cured siloxane polymers. J. Appl. Polym. Sci. 1975, 19, 2817-2820. [CrossRef]

29. Brook, M.A.; Zhao, S.; Liu, L.; Chen, Y. Surface etching of silicone elastomers by depolymerization. Can. J. Chem. 2012, 90, 153-160. [CrossRef]

30. Qiang, Z.; Gurkan, B.; Ma, J.; Liu, X.; Guo, Y.; Cakmak, M.; Cavicchi, K.A.; Vogt, B.D. Roll-to-roll fabrication of high surface area mesoporous carbon with process-tunable pore texture for optimization of adsorption capacity of bulky organic dyes. Microporous Mesoporous Mater. 2016, 227, 57-64. [CrossRef]

31. Selva, M.; Fabrisa, M.; Perosa, A. Decarboxylation of dialkyl carbonates to dialkyl ethers over alkali metal-exchanged faujasites. Green Chem. 2011, 13, 863-872. [CrossRef]

32. Tundo, P.; Perosa, A.; Zecchini, F. Methods and Reagents for Green Chemistry: An Introduction, 1st ed.; Willey: Venezia, Italy, 2007; 336p, ISBN 978-0-471-75400-8.

33. Ono, Y.; Akiyama, M.; Suzuki, E. Direct Synthesis of Tetraalkoxysilanes from Silica by Reaction with Dialkyl Carbonates. Chem. Mater. 1993, 5, 442-447. [CrossRef]

34. Okamoto, M.; Suzuki, S.; Suzuki, E. Polysiloxane depolymerization with dimethyl carbonate using alkali metal halide catalysts. Appl. Catal. A 2004, 261, 239-245. [CrossRef]

35. Demianenko, E.M.; Grebenyuk, A.G.; Lobanov, V.V.; Protsak, I.S.; Kozakevych, R.B.; Bolbukh, Y.M.; Tertykh, V.A. Quantum chemical study on interaction of dimethyl carbonate with polydimethylsiloxane. Chem. Phys. Technol. Surf. 2014, 5, 473-479.

36. Protsak, I.S.; Tertykh, V.A.; Pakhlov, E.M.; Derylo-Marczewska, A. Modification of fumed silica surface with mixtures of polyorganosiloxanes and dialkyl carbonates. Prog. Org. Coat. 2017, 106, 163-169. [CrossRef]

37. Gun'ko, V.M.; Pakhlov, E.M.; Goncharuk, O.V.; Andriyko, L.S.; Marynin, A.I.; Ukrainets, A.I.; Charmas, B.; Skubiszewska-Zieba, J.; Blitz, J.P. Influence of hydrophobization of fumed oxides on interactions with polar and nonpolar adsorbates. Appl. Surf. Sci. 2017, 423, 855-868. [CrossRef]

38. Gun'ko, V.M.; Turov, V.V. Nuclear Magnetic Resonance Studies of Interfacial Phenomena (Surfactant Science), 1st ed.; CRC Press: Boca Raton, FL, USA, 2013; 1040p, ISBN 978-1466551688.

39. Legrand, A.P. (Ed.) The Surface Properties of Silicas, 1st ed.; Wiley: New York, NY, USA, 1998; 494p, ISBN 978-0471953326.

40. Kiselev, A.V.; Lygin, V.I. Infrared Spectra of Surface Compounds, 1st ed.; Wiley: New York, NY, USA, 1975; 384p, ISBN 978-0470489055.

41. Hair, M.L. Infrared Spectroscopy in Surface Chemistry, 1st ed.; Dekker: New York, NY, USA, 1967; 336p, ISBN 978-0824712853.

(C) 2018 by the authors. Licensee MDPI, Basel, Switzerland. This article is an open access article distributed under the terms and conditions of the Creative Commons Attribution (CC BY) license (http:/ / creativecommons.org/licenses/by/4.0/). 


\title{
Article \\ PNIPAM-MAPOSS Hybrid Hydrogels with Excellent Swelling Behavior and Enhanced Mechanical Performance: Preparation and Drug Release of 5-Fluorouracil
}

\author{
Peihong Li, Xiaoman Hou, Lijie Qu, Xueyan Dai and Chunling Zhang * \\ Key Laboratory of Automobile Materials, Ministry of Education, College of Materials Science and Engineering, \\ Jilin University, Changchun 130022, China; phli16@mails.jlu.edu.cn (P.L.); houxm1205@163.com (X.H.); \\ qulijie92528@outlook.com (L.Q.); godjealouselite@163.com (X.D.) \\ * Correspondence: clzhang@jlu.edu.cn; Tel.: +86-431-8509-5170
}

Received: 14 January 2018; Accepted: 29 January 2018; Published: 31 January 2018

\begin{abstract}
Poly(N-isopropylacrylamide) (PNIPAM) is a widely-studied polymers due to its excellent temperature sensitivity. PNIPAM-MAPOSS hybrid hydrogel, based on the introduction of acrylolsobutyl polyhedral oligomeric silsesquioxane (MAPOSS) into the PNIPAM matrix in the presence of polyethylene glycol, was prepared via radical polymerization. The modified hydrogels exhibited a thick, heterogeneous porous structure. PEG was used as a pore-forming agent to adjust the pore size. MAPOSS reduced the swelling ratios of gels, and decreased the LCST, causing the hydrogels to shrink at lower temperatures. However, its hydrophobicity helped to improve the temperature response rate. The incorporation of rigid MAPOSS into the polymer network greatly increased the compressive modulus of the hydrogel. It is worth noting that, by adjusting the amount of MAPOSS and PEG, the hydrogel could have both ideal mechanical properties and swelling behavior. In addition, hydrogel containing $8.33 \mathrm{wt} \%$ MAPOSS could achieve stable and sustained drug release. Thus, the prepared PNIPAM-MAPOSS hybrid hydrogel can serve as drug carrier for 5 -fluorouracil and may have potential application in other biomedical fields.
\end{abstract}

Keywords: hybrid hydrogel; MAPOSS; mechanical properties; swelling; drug release

\section{Introduction}

Intelligent hydrogels have attracted considerable attention because of their abilities to change its volume and property in response to external stimuli, such as temperature [1], $\mathrm{pH}$ [2], light [3], ionic strength [4], electric fields [5], and magnetic fields [6]. Among all these stimuli-sensitive hydrogels, poly( $N$-isopropylacrylamide) (PNIPAM) is one of the widely-studied polymers due to its excellent temperature sensitivity. A series of studieshave been made to research its potential application, such as dye absorption [7,8], tissue engineering [9], and drug delivery [10-12]. The PNIPAM-based hydrogels undergo an abrupt volume phase transition at a lower critical solution temperature (LCST) of around $32{ }^{\circ} \mathrm{C}$ [13]. During the drug delivery, PNIPAM hydrogels swell and load the drug at a temperature below LCST, and shrink and release the loaded drug when the temperature rises above LCST. Typical types of polymerization are free radical polymerization and reversible addition-fragmentation chain transfer polymerization (RAFT).Polymerization methods include solution polymerization, inverse microemulsion polymerization [14], inverse suspension polymerization [15], precipitation polymerization [16], etc.

However, the slow temperature response rate and the low mechanical strength are common disadvantages of hydrogels. There are many general methods to modify hydrogels, including cross-linking, nanocomposites [17], porous structures [18], IPNs [19,20], etc. Polyhedral oligomeric silsesquioxane 
(POSS) with a cubic cage-like nanostructure is emerging as a promising organic-inorganic nanoparticle for the creation of nanocomposites. It serves as a terminal or cross-linking curing center to meet the different needs of modified polymers. POSS-polymer nanocomposite combination property is excellent. The main functions of POSS are as follows: (1) to improve the thermal performance, thereby increasing the operating temperature of composite materials can withstand; (2) to strengthen the mechanical properties, which is mainly due to the rigid structure of POSS; (3) to improve the processing performance; (4) to improve flame retardancy, make the composite material have a significant delayed combustion characteristics; and (5) to make it multi-functional. POSS modified hydrogels generally have good mechanical properties, response rate and thermal stability [21-25]. There are many kinds of such nanoparticles. The MAPOSS used in this paper carries carbon-carbon double bond functional groups. Therefore, it can be introduced into the matrix by cross-linking.

We have synthesized a series of MAPOSS-incorporated P(NIPAM-co-PEGDA) hybrid hydrogels in previous work [26].The introduction of POSS can improve some properties of hydrogels, especially mechanical properties, but this also weakens the water retention. In this paper, PNIPAM was still used as a matrix, a small amount of PEGDA as a crosslinking agent. We prepared a PNIPAM-MAPOSS hybrid hydrogel, and introduced a pore-forming agent PEG to adjust the pore structure. We focused on the swelling behavior and mechanical properties of this hydrogel. Then we studied its controlled release of the anti-cancer drug 5-fluorouracil.The results show that, the prepared gels had temperature sensitivity, excellent swelling behavior, enhanced mechanical properties, and potential application in drug delivery system.

\section{Experimental Section}

\subsection{Materials}

Acrylolsobutyl polyhedral oligomeric silsesquioxane (MAPOSS) was purchased from Hybrid Plastics Co. (Hattiesburg, MS, USA). N-isopropylacrylamide (NIPAM), polyethylene glycol diacrylate (PEGDA, average molecular weight 200) and 5-fluorouracil (5-FU) were Aladdin (Shanghai, China) products. Polyethylene glycol (PEG 4000), 2,2'-azobis(2-methylpropionitrile) (AIBN), and tetrahydrofuran (THF) were Sinopharm (Shanghai, China) products. PEG 4000 and AIBN are chemical pure and THF is analytically pure. All reagents were used as received. Phosphate buffer solution (PBS, pH 7.4) was prepared using potassium dihydrogen phosphate $(1.36 \mathrm{~g})$ and $\mathrm{NaOH}$ solution $(0.1 \mathrm{~mol} / \mathrm{L}$, $79 \mathrm{~mL}$ ), followed by diluting the solution to $200 \mathrm{~mL}$ with ultrapure water.

\subsection{Preparation of PNIPAM-MAPOSS Hybrid Hydrogels}

All types of PNIPAM-MAPOSS hybrid hydrogels were prepared by one-step radical polymerization. The synthesis process can refer to our previous work [26].Briefly, $1 \mathrm{~g}$ NIPAM and $0.10 \mathrm{~g}$ PEGDA with different proportions of MAPOSS and PEG were dissolved in THF at room temperature. Then $0.01 \mathrm{~g}$ AIBN was added to the mixture dispersion with stirring. The resulting solution was transferred to a glass tube and placed in a water bath at $60{ }^{\circ} \mathrm{C}$. All samples were taken out after the reaction, and immersed in THF and deionized water alternately for threedays to remove PEG and impurities. A schematic is shown in Figure 1. The detailed feed ratios of each group of PNIPAM-MAPOSS hybrid hydrogels are listed in Table 1.

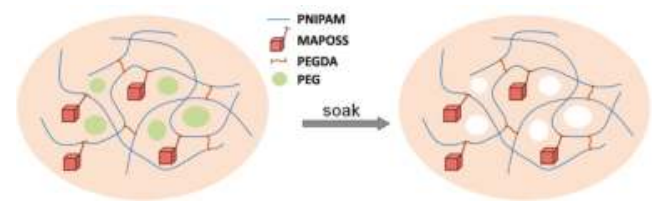

Figure 1. Schematic of preparation of PNIPAM-MAPOSS hybrid hydrogels. 
Table 1. PNIPAM-MAPOSS hybrid hydrogels composition and LCSTs.

\begin{tabular}{ccccc}
\hline \multirow{2}{*}{ Sample } & \multicolumn{2}{c}{ Gels Formulation $(\mathbf{g})^{\mathbf{1}}$} & \multirow{2}{*}{ MAPOSS (wt \%) } & LCST $\left({ }^{\circ} \mathbf{C}\right)^{2}$ \\
\cline { 2 - 3 } & MAPOSS & PEG & 0 & 31.1 \\
Gel 1 & 0 & 0.10 & 4.35 & 31.1 \\
Gel 2 & 0.05 & 0.10 & 8.33 & 31.1 \\
Gel 3 & 0.10 & 0.10 & 15.38 & 29.4 \\
Gel 4 & 0.20 & 0.10 & 21.43 & 28.5 \\
Gel 5 & 0.30 & 0.10 & 8.33 & 31.1 \\
Gel 6 & 0.10 & 0.05 & 8.33 & 31.1 \\
Gel 7 & 0.10 & 0.30 & 8.33 & 31.1 \\
Gel 8 & 0.10 & &
\end{tabular}

\subsection{Characterization}

Fourier-transform infrared spectrometry (FTIR) spectra of hydrogels were recorded using $\mathrm{KBr}$ disks on a Nicolet Nexus 670 spectrometer (Nicolet, Madison, WI, USA). In all case, the scans were carried out in the spectral range varying from 4000 to $400 \mathrm{~cm}^{-1}$ with a resolution of $4 \mathrm{~cm}^{-1}$. The interior morphologies of the freeze dried specimens were observed by environmental scanning electron microscopy (ESEM; XL30 ESEM-FEG, FEI, Hillsboro, OR, USA) after sputter-coating with gold under vacuum. The LCST measurement of the wet samples from 20 to $50{ }^{\circ} \mathrm{C}$ was determined by differential scanning calorimetry (DSC; Q20, TA, New Castle, PA, USA) under a nitrogen atmosphere with a heating rate of $2^{\circ} \mathrm{C} / \mathrm{min}$.

\subsection{Swelling Equilibrium of the PNIPAM-MAPOSS Hybrid Hydrogels}

The pre-weighed dry hydrogels were immersed in deionized water at 20 to $50{ }^{\circ} \mathrm{C}$ until swelling equilibrium was reached. The weight of hydrogel was measured after removing the excess water on the surface with wet filter paper. The swelling ratio $(S R)$ was determined by the gravimetric method and calculated by following Equation (1):

$$
S R=\frac{W_{\mathrm{s}}-W_{\mathrm{d}}}{W_{\mathrm{d}}},
$$

where $W_{\mathrm{s}}$ is the weight of the swollen hydrogel reached swelling equilibrium at a given temperature, and $W_{\mathrm{d}}$ is the weight of dry hydrogel [27].

The deswelling behavior was investigated by recording the change in the weight of water in the hydrogels over time. The hydrogels samples first reached swelling equilibrium at $25^{\circ} \mathrm{C}$, and were then placed in water at $60^{\circ} \mathrm{C}$. The samples were recorded at regular time intervals. Water retention (WR) corresponding to the deswelling ratio is defined by Equation (3):

$$
W R=\frac{W_{\mathrm{f}}-W_{\mathrm{d}}}{W_{\mathrm{s}}-W_{\mathrm{d}}} \times 100 \%,
$$

where $W_{\mathrm{f}}$ is the weight of the hydrogel at a certain time, $W_{\mathrm{s}}$ is the ultimate weight of the swollen hydrogel reached swelling equilibrium at $25{ }^{\circ} \mathrm{C}$, and $W_{\mathrm{d}}$ is the weight of dry hydrogel [28]. Five independent samples were tested and plotted with the average of their $S R_{\mathrm{S}}$ and $W R_{\mathrm{s}}$.

\subsection{Mechanical Performance Test}

The mechanical properties of PNIPAM-MAPOSS hybrid hydrogels were investigated with a WSM universal mechanical tester (Changchun Intelligent Equipment Co., Ltd., Changchun, China) at room temperature. The cylindrical samples (10 $\mathrm{mm}$ in diameter, $10 \mathrm{~mm}$ in height) were immersed in deionized water and kept at $25^{\circ} \mathrm{C}$ for at least $24 \mathrm{~h}$ prior to testing. 
Since the hydrogels are soft materials, all the samples were compressed at a rate of $10 \mathrm{~mm} / \mathrm{min}$ until the compression ratio reached $70 \%$. According to the obtained stress-strain curves, the hydrogel compressive tangent modulus was calculated by the finite difference method at $5 \%$ and $20 \%$ strain. The equation is follows:

$$
E_{\varepsilon}=\frac{\sigma_{\varepsilon+\Delta \varepsilon}-\sigma_{\varepsilon}-\Delta \varepsilon}{2 \Delta \varepsilon},
$$

Where $E_{\varepsilon}$ is the compressive tangent modulus of the hydrogel at the compressive strain ratio of $\varepsilon$. $\Delta \varepsilon$ is the variable of the strain, taking $2 \%$ [29]. In addition, five independent samples were tested and the average was calculated.

\subsection{Drug Release Behavior of 5-Fluorouracil}

The drug-loaded hydrogels were prepared by using 5-FU as a model drug. The disc-like dried hydrogel was immersed in 5-FU solution $(1 \mathrm{mg} / \mathrm{mL})$ at $25^{\circ} \mathrm{C}$ for $48 \mathrm{~h}$ to reach swelling equilibrium, and then dried using vacuum freezing to obtain a drug-loaded hydrogel. The in vitro release behavior of 5-FU from PNIPAM-MAPOSS hybrid hydrogels was studied in PBS ( $\mathrm{pH} \mathrm{7.4)} \mathrm{at} \mathrm{physiological}$ temperature. The PBS was shocked at $100 \mathrm{rpm}$. At each fixed time interval, $3 \mathrm{~mL}$ solution was taken out and replaced by $3 \mathrm{~mL}$ of fresh PBS to maintain the volume of the medium. There are five independent samples for each kind of gel for testing. The released amount of 5-FU was measured by ultraviolet spectrophotometer (UV; UV-6100s, MAPADA, Shanghai, China) at $265 \mathrm{~nm}$ [30]. The average of released amounts was calculated for each group.

\section{Results and Discussion}

\subsection{FTIR Spectroscopy}

The FTIR spectra of PNIPAM-MAPOSS hybrid hydrogels are shown in Figure 2. The characteristic peaks of NIPAM at 1659 and $1549 \mathrm{~cm}^{-1}$ are assigned to the $\mathrm{C}=\mathrm{O}$ stretching vibration and $\mathrm{N}-\mathrm{H}$ deformation vibration of amide group. The typical peak appearing at $1747 \mathrm{~cm}^{-1}$ belongs to the $\mathrm{C}=\mathrm{O}$ stretching vibration of ester group in PEGDA and MAPOSS. The peaks at 1622, 1630, and $1629 \mathrm{~cm}^{-1}$, related to the $\mathrm{C}=\mathrm{C}$ stretching vibrations in NIPAM, PEGDA and MAPOSS macromer, disappeared in PNIPAM-MAPOSS hybrid hydrogels (Gel 1, 3, 6), indicating chemical cross-linking between the three. The peaks of Si-O-Si and Si-C of MAPOSS at 1111 and $743 \mathrm{~cm}^{-1}$ could be found in Gel 3 and 6 , indicating the successful incorporation of MAPOSS into Gel 3 and 6, but not Gel 1. It should be pointed out that, the absence of terminal hydroxyl at $3445 \mathrm{~cm}^{-1}$ in hybrid hydrogels confirmed the remove of PEG. PEG only acted as a pore-forming agent and did not participate in the reaction.

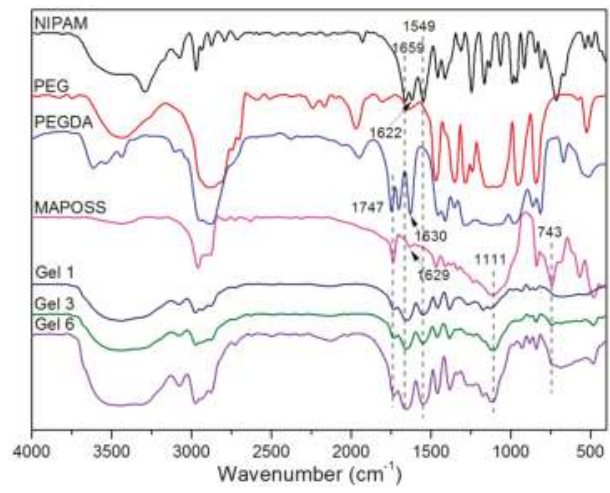

Figure 2. FTIR spectra of NIPAM, PEG, PEGDA, MAPOSS, and PNIPAM-MAPOSS hybrid hydrogels. 


\subsection{Interior Morphology}

The SEM images were used to study the effect of MAPOSS and PEG on the porous structure of gels. Figure 3 shows the interior pore of these hydrogels. Gel 1 without MAPOSS exhibited a porous morphology with a very thin wall, which is shown in detail in Figure $3 \mathrm{a}$. The pores of Gel 2 became larger and heterogeneous than Gel 1 due to the introduction of 4.35 wt \% MAPOSS. It can be directly observed from the SEM images that the pore size decreased as the MAPOSS content increased (average pore size: Gel $2>$ Gel $3>$ Gel $4>$ Gel 5). The distinct cubic core $(0.53 \mathrm{~nm})$ and the functionalized arms of MAPOSS occupied some space in the network structure. Therefore, the pore walls of the hybrid hydrogels became thicker and their structural homogeneity deteriorated. The formation of a heterogeneous, porous structure in hydrogel is usually ascribed to polymerization-induced microphase-separation. MAPOSS and hydrophobic portions of PNIPAM self-assembled into hydrophobic domains. These hydrophobic MAPOSS domains would also act as cross-linking sites connecting PNIPAM chains, so that the increase of MAPOSS made a more compact network structure, leading the pore size reduced. On the contrary, it is evident that the pore diameter of Gel 6 without PEG was only $200 \mathrm{~nm}$, while the Gel 8 exhibited macroporous structure with pore diameter of 3 to $7 \mu \mathrm{m}$ (see Figure S1). The amount of pore-forming agent PEG was directly related to the pore size (average pore size: Gel $8>\operatorname{Gel} 3>$ Gel $7>$ Gel 6). After the polymerization, we soaked gels in THF and deionized water alternately to remove PEG. The original position occupied by PEG became a pore, and these pores were regular in size.

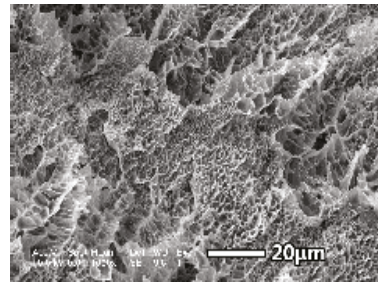

(a)

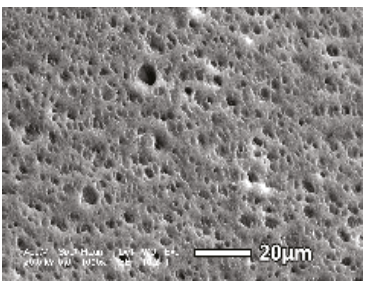

(d)

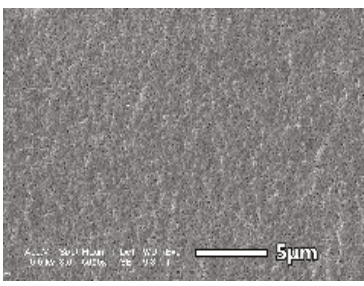

(g)

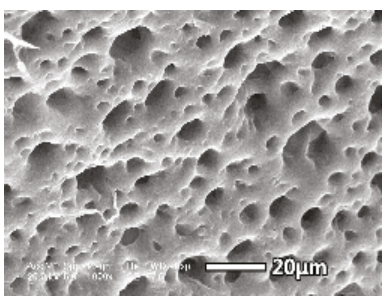

(b)

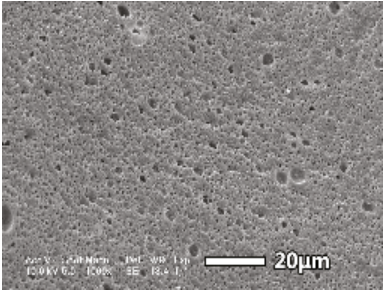

(e)

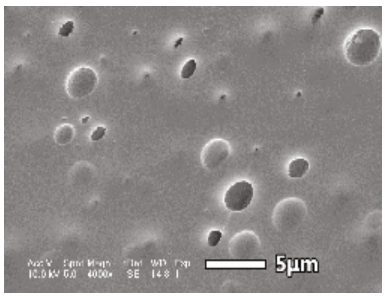

(h)

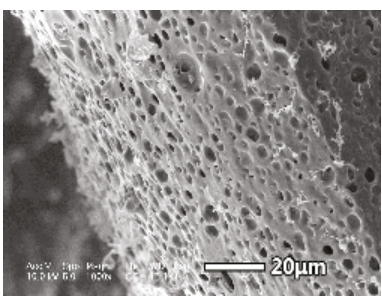

(c)

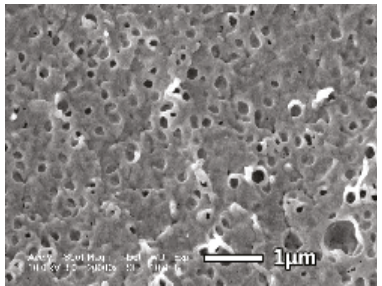

(f)

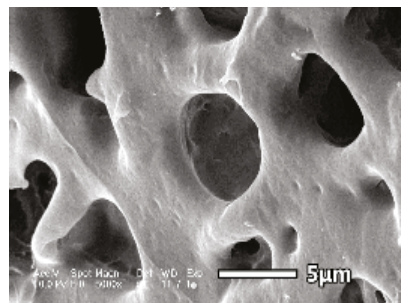

(i)

Figure 3. SEM images of (a) Gel 1; (b) Gel 2; (c) Gel 3; (d) Gel 4; (e) Gel 5; (f,g) Gel 6; (h) Gel 7; and (i) Gel 8 . 


\subsection{Volume Phase Transition Behavior}

PNIPAM-based hydrogels are temperature sensitive. In essence, the volume change of the temperature-sensitive hydrogel in the sense of temperature changes is a phase separation process, so that the phase separation heat is accompanied. The DSC thermograms of hydrogels are shown in Figure 4, and the LCSTs can be found in Table 1. We defined the endothermic peak on the curve as the LCST.

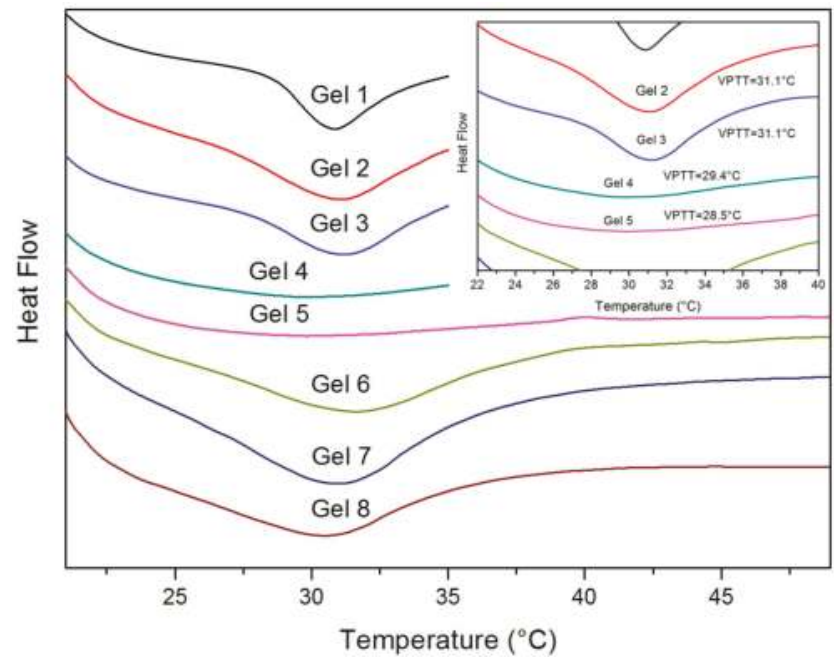

Figure 4. The phase transition behavior of gels measured by DSC.

The results revealed that, when the amount of MAPOSS increased from 0 to 21.43 wt \%, the LCSTs decreased from 31.1 to $28.5^{\circ} \mathrm{C}$. PNIPAM has inherently hydrophilic segments and hydrophobic segments, but this hydrophilic-hydrophobic balance of hydrogel was changed by adding MAPOSS. With more hydrophobic components, the affinity of the gels with water reduced and the LCST decreased. Even the endothermic peaks of Gel 4 and Gel 5 became insignificant. Too much MAPOSS weakened the temperature sensitivity of hydrogels. Hence, PNIPAM-MAPOSS hybrid hydrogels could shrink at a lower temperature. In addition, the use of PEG to prepare hydrogels had little impact on its LCST.

\subsection{Swelling and Deswelling Behavior}

The swelling behavior was used to study the temperature sensitivity of gels, while the deswelling behavior was used to study the response rate. The temperature-dependent swelling ratios (SRs) of hydrogels were investigated at different temperatures, as shown in Figure 5a. All samples had the volume phase transition behavior and exhibited excellent thermal responsiveness, supported by their SRs reduction, especially between 22.0 and $34.0^{\circ} \mathrm{C}$. At temperatures above LCST, the SRs were relatively small, but not very different, because the hydrogels shrunk at high temperatures and the network structure became similar.

MAPOSS was not conducive to swelling. From the SEM images, we already know that the MAPOSS caused the internal pores of the hydrogel smaller and heterogeneous. Both the tight network structure and hydrophobicity reduced the SRs of gels. However, it was intuitively observed in Figure 5a that the addition of hydrophobic MAPOSS allowed the hydrogels to shrink at lower temperatures. As shown in Figure 5b, the relationship between the deswelling rate and the MAPOSS content was varied. Gel 2 reached equilibrium at $180 \mathrm{~min}$, which deswelling rate was faster than Gel 1 because of 
the incorporation of MAPOSS. The hydrophobic domains could behave as the micro-porogens and, therefore, the contact area between polymer chains and water molecules were significantly increased. This would facilitate the diffusion of water molecules in the cross-linked networks. However, the deswelling rate became slower when the content of MAPOSS reached $15.38 \mathrm{wt} \%$ (Gel 4). After analysis, too much MAPOSS made the internal pores of hydrogel very small to limit water loss.

The introduction of PEG could significantly change the size of the pores inside the gels, thereby affecting the swelling behavior. The SR of Gel 8 (with the highest PEG content) was apparently higher than others. Larger internal pores could accommodate more water, so that the more the amount of PEG used, the higher the gel's $S R$. Gel 8 also exhibited the fastest deswelling rate in Figure 5b. Gel 8 lost more than $75 \%$ water within $90 \mathrm{~min}$, while only $40 \%$ water was evacuated from Gel 6 . All the comparative tests showed that, the hydrogel with big internal pore had a large swelling ratio, fast deswelling rate, but poor water retention capacity. In summary, the temperature response behavior of the hydrogel was not only related to the structure of the pores, but also to the hydrophobicity of the substance. Hybrid hydrogel with a suitable swelling ratio and response rate could be prepared by co-adjusting the amounts of MAPOSS and PEG.
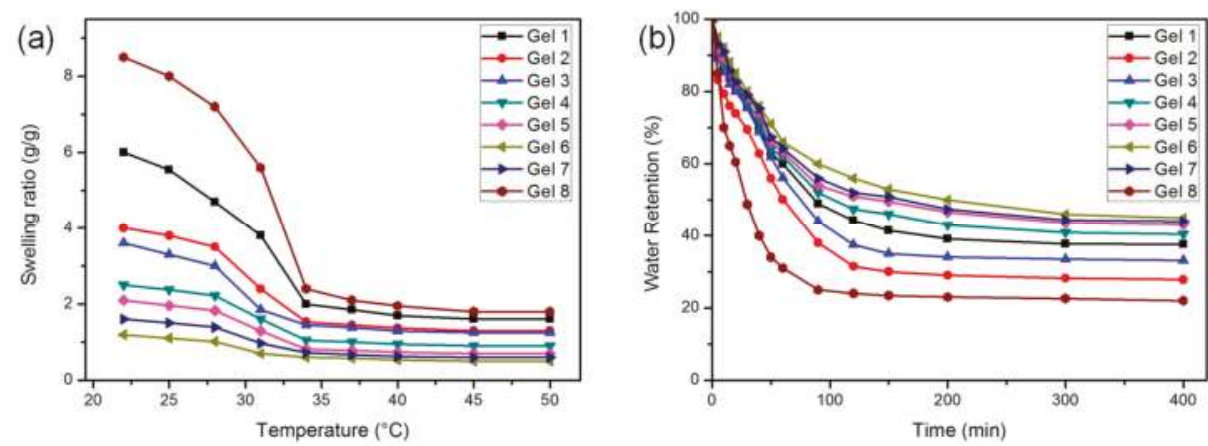

Figure 5. (a) The temperature-dependent swelling ratios of gels between 20 and $50{ }^{\circ} \mathrm{C}$; and (b) deswelling behavior of gels at $60^{\circ} \mathrm{C}$.

\subsection{Mechanical Performance}

The compression stress-strain curves of gels were shown in Figure 6a. Figure $6 \mathrm{~b}, \mathrm{c}$ show the effect of MAPOSS and PEG on the compressive modulus of hydrogels, respectively. It was shown that the compressive tangent modulus $\left(E_{\varepsilon}\right)$ calculated at $5 \%$ and $20 \%$ strain followed the same tend. In Figure 6b, Gel 1 exhibited a poor compressive performance (only $0.1 \mathrm{MPa}$ at $5 \%$ strain, $0.3 \mathrm{MPa}$ at $20 \%$ strain), while the $E_{\varepsilon}$ of Gel 5 had been dramatically increased to approximately 10 times (about $2.1 \mathrm{MPa}$ at $5 \%$ strain, and $2.8 \mathrm{MPa}$ at $20 \%$ strain). Only by adding $8.33 \mathrm{wt} \%$ MAPOSS in the hydrogels could make the $E_{\varepsilon}$ of Gel 3 reach $0.5 \mathrm{MPa}$ at $5 \%$ strain and $0.7 \mathrm{MPa}$ at $20 \%$ strain. The increase of compressive modulus could be explained mainly by three reasons. Firstly, the incorporation of rigid inorganic POSS core into the hydrogel networks enhanced the hardness of polymer chains. Secondly, MAPOSS with double bonds played the role of a cross-linking agent. It increased the cross-linking degree of gels and made the network more robust. Finally, the dispersion of POSS cages at the nanometer level could significantly restrict the motions of macromolecular chains as shown in other POSS-reinforced polymers. MAPOSS was easy to cause polymers chains entanglement, also helped to improve the mechanical properties of hydrogels. 

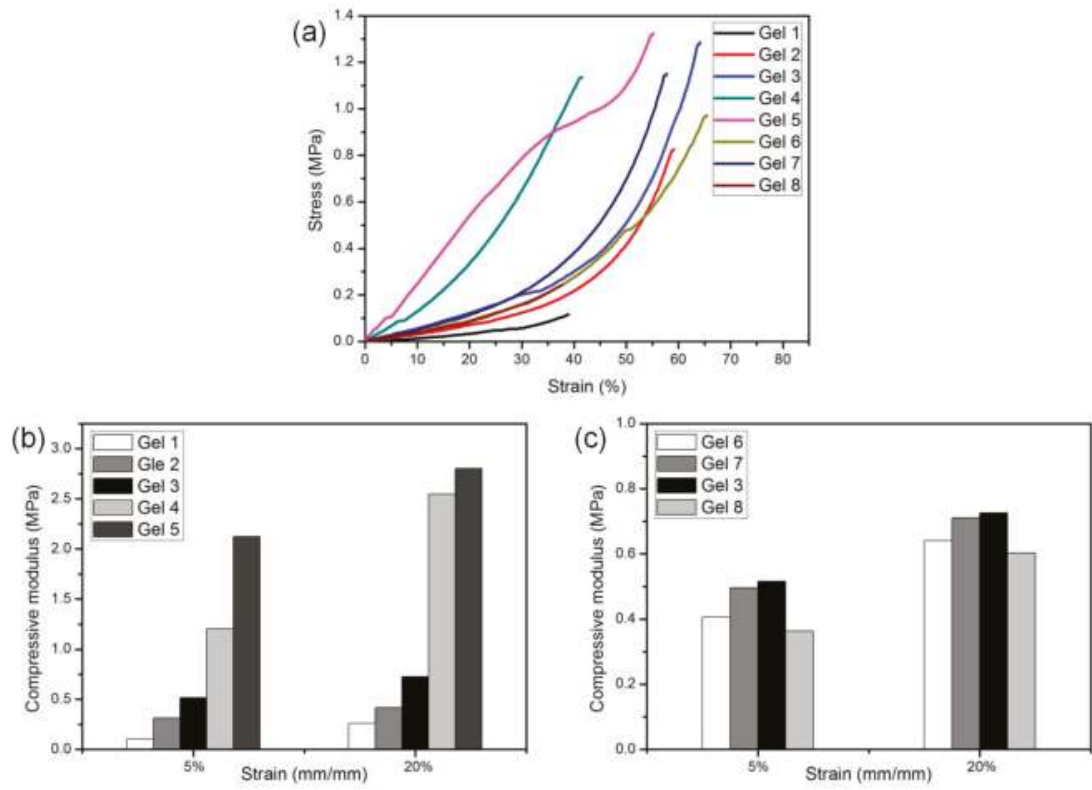

Figure 6. (a) Compression performance of gels: compression stress-strain curves; and (b,c) compressive tangent modulus at $5 \%$ strain and $20 \%$ strain.

As shown in Figure 6c, with the increase of PEG content, the $E_{\varepsilon}$ increased first and then decreased. Appropriate water absorption could better maintain the network structure of hydrogel. Hence, it had good mechanical properties. However, larger pore size and higher $S R$ caused by too much PEG were counterproductive. However, the effect of PEG on the mechanical properties of gels was not as obvious as that of MAPOSS. The improved hydrogels were more stable and could withstand a certain pressure without being destroyed.

\subsection{Drug Release of 5-Fluorouracil}

5-Fluorouracil (5-FU) is an early anti-cancer drug and the most widely used in clinical anti-pyrimidine drugs [6]. It has a good therapeutic effect on gastrointestinal cancer and other solid tumors. However, its rapid metabolism in body may reduce the therapeutic effect [31]. Hsiue et al. synthesized PLA- $g$-P(NIPAm-co-MAA) nanoparticles with core-shell structure were thremo-responsive, $\mathrm{pH}$-responsive and biodegradable. The drug loading level of 5-FU encapsulated in this nanoparticles could reach $20 \%$ [32]. Dias et al. synthesized molecularly imprinted particles with surface grafted functional polymer brushes aiming at the targeting of 5-FU. For particles with PNIPA grafted brushes, a boost in drug release was also shown at $20^{\circ} \mathrm{C}$ as compared to $40^{\circ} \mathrm{C}[15,33]$. In this work, the release behaviors of the 5-FU loaded NIPAM-MAPOSS hydrogels in PBS (pH 7.4) were evaluated at $37^{\circ} \mathrm{C}$ for $9 \mathrm{~h}$. The solubility of 5-FU in saline solution is about $1.45 \mathrm{~mol} / \mathrm{L}$ at $37^{\circ} \mathrm{C}$ [34]. All types of gels functioned as drug delivery systems that control the release of 5-FU.We selected several representative examples to investigate the effect of MAPOSS and PEG on the release efficiency and sustained release time of hydrogel. The cumulative release percentage of these groups of gels was listed in Table S1. The data for drug release (time from 1 to $9 \mathrm{~h}$ ) were fitted to a logarithmic function as follows:

$$
y=k \ln (x)+b,
$$


where $k$ affects the slope of the curve, $b$ is directly related to the value of $y$, and $y$ represents the cumulative release. The value of $k$ is larger, the drug release is gentle. The release curves and the functions were shown in Figure 7.

The curves of Gel 1, Gel 3, and Gel 5 reflect the effect of MAPOSS on drug release performance. The final cumulative release of Gel 1 and Gel 3 are close, more than $70 \%$. At the initial stage, the release rate of Gel 1 (without MAPOSS) was fast. Meanwhile, the cumulative release percentage of Gel 3 (containing $8.33 \mathrm{wt} \%$ MAPOSS) was lower than Gel 1, and then achieved a steady, sustained release in $300 \mathrm{~min}$. Gel 3 had an ideal release rate and release efficiency. However, when the amount of MAPOSS continued to increase in the feed, the cumulative release percentage of Gel 5 became low. This was because the unique spatial structure of MAPOSS was conducive to the storage and slow release of drugs. MAPOSS also made the polymer network structure more solid, so as to achieve a sustained and stable release. However, too tight network structure limited the water in the hydrogel in and out, was against to drug loading and release. The results demonstrate that the addition of appropriate amount of MAPOSS can prolong the release time while maintaining the cumulative release percentage. Moreover, the curves of Gel 3, Gel 6, and Gel 8 show the effect of PEG on drug release performance of gels. The Gel 8 release curve was above any other curve. Nearly $76 \%$ of the 5-FU adsorbed in Gel 8 was released in $180 \mathrm{~min}$, while Gel 6 only released 39\% over the same time. And after $240 \mathrm{~min}$, Gel 8 was barely released. The simulated curve of Gel 3 had a larger value of $\mathrm{k}$ than the others. The $b$ value of the simulated release curve decreased as the amount of MAPOSS increases and increased with the amount of PEG. We can conclude that the release efficiency of macroporous hydrogel is high, but the duration of the sustained release is short.

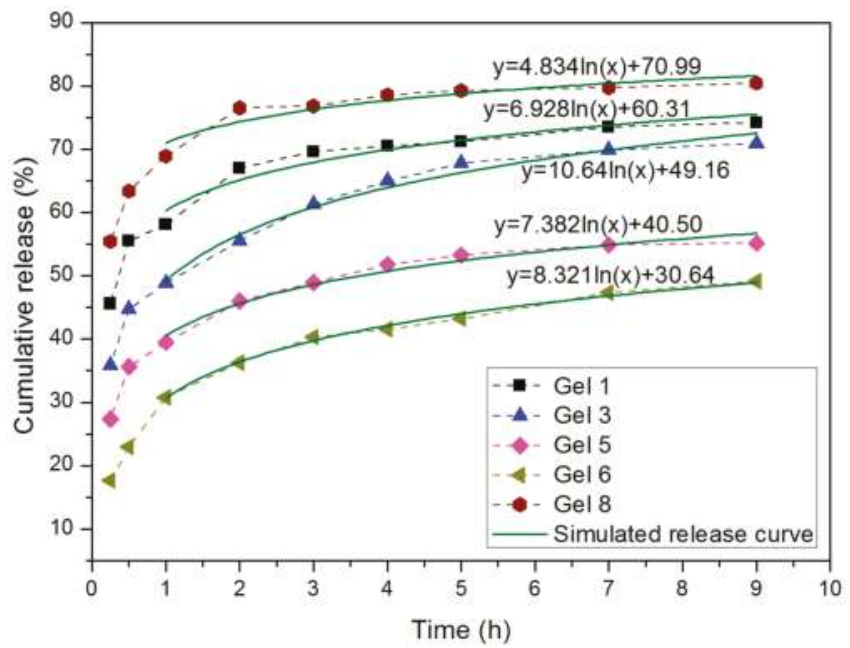

Figure 7. The release behaviors of 5-FU from gels in PBS ( $\mathrm{pH} 7.4)$ at $37^{\circ} \mathrm{C}$ and the simulated release curves.

\section{Conclusions}

A series of PNIPAM-MAPOSS hybrid hydrogels were prepared by one-step radical polymerization and utilized for drug delivery. From the morphology, the addition of MAPOSS made the internal pores heterogeneous and the network tight. Microphase-separation could be observed. The hydrophobic MAPOSS domains functioned as cross-linking sites, linking PNIPAM chains. The amount of PEG directly determined the size of pores. The PNIPAM-MAPOSS hybrid hydrogels were temperature sensitive because of NIPAM. However, adding MAPOSS decreased its LCST. The tight network structure and hydrophobicity reduced the SRs of gels. However, the hydrophobic domains could 
behave as the micro-porogens to facilitate the diffusion of water molecules, thereby increasing the temperature response rate of the hydrogels. The incorporation of rigid MAPOSS into the hydrogels greatly improved their compressive modulus. Just adding a small amount of MAPOSS could increase the compressive modulus of hydrogel approximately 10 times. This can be explained as MAPOSS increased the cross-linking degree of gels and made the network more robust. Additionally, the dispersion of POSS cages limited the movement of the polymer chains. The right amount of MAPOSS also helped to prolong the sustained release of drugs. MAPOSS was conducive to the storage and sustained release of drugs, and made the polymer network difficult to be destroyed in the water. PEG essentially affected the performance of hydrogels by changing the size of the pores. The large-pore hydrogel had a high swelling ratio, fast deswelling rate, and greater drug loading. However, its loaded drug released quickly and its mechanical properties might be weakened. More importantly, we obtained Gel 3 by adjusting the amount of MAPOSS and PEG, had a moderate pore size, excellent swelling behavior, and enhanced mechanical properties. It can serve as a drug carrier for 5-FU to treat cancer, but also for other biomedical applications.

Supplementary Materials: The following are available online at www.mdpi.com/2073-4360/10/2/137/s1, Figure S1: The pore size distribution of PNIPAM-MAPOSS hybrid hydrogels: (a) Gel 6, (b) Gel 7, (c) Gel 3, and (d) Gel 8; Table S1: The cumulative release percentage (\%) of Gel 1, Gel 3, Gel 5, Gel 6, and Gel 8.

Acknowledgments: The authors gratefully acknowledge the financial supports for this research from the Development Project of Jilin provincial Science and Technology of China (No. 20180101197jc).

Author Contributions: Chunling Zhang and Xiaoman Hou conceived and designed the experiments; Peihong Li and Xiaoman Hou performed most of the experiments, Xueyan Dai was in charge of the mechanical part; Peihong Li and Lijie Qu analyzed the data and plotted the figures. Peihong Li wrote the paper, and Chunling Zhang provided revise the manuscript.

Conflicts of Interest: The authors declare no conflict of interest.

\section{References}

1. Reddy, N.N.; Ravindra, S.; Reddy, N.M.; Rajinikanth, V.; Raju, K.M.; Vallabhapurapu, V.S. Temperature responsive hydrogel magnetic nanocomposites for hyperthermia and metal extraction applications. J. Magn. Magn. Mater. 2015, 394, 237-244. [CrossRef]

2. Marambio, O.G.; Pizarro, G.D.C.; Jeria-Orell, M.; Geckeler, K.E. Swelling behavior and metal ion retention from aqueous solution of hydrogels based on $N$-1-vinyl-2-pyrrolidone andn-hydroxymethylacrylamide. J. Appl. Polym. Sci. 2009, 113, 1792-1802. [CrossRef]

3. Cevik, O.; Gidon, D.; Kizilel, S. Visible-light-induced synthesis of pH-responsive composite hydrogels for controlled delivery of the anticonvulsant drug pregabalin. Acta Biomater. 2015, 11, 151-161. [CrossRef] [PubMed]

4. Jia, X.; Wang, K.; Wang, J.; Hu, Y.; Shen, L.; Zhu, J. Full-color photonic hydrogels for pH and ionic strength sensing. Eur. Polym. J. 2016, 83, 60-66. [CrossRef]

5. Yang, S.; Liu, G.; Wang, X.; Song, J. Electroresponsive behavior of a sulfonated poly(vinyl alcohol) hydrogel and its application to electrodriven artificial fish. J. Appl. Polym. Sci. 2010, 117, 2346-2353. [CrossRef]

6. Li, L.; Chen, L.; Zhang, H.; Yang, Y.; Liu, X.; Chen, Y. Temperature and magnetism bi-responsive molecularly imprinted polymers: Preparation, adsorption mechanism and properties as drug delivery system for sustained release of 5-fluorouracil. Mater. Sci. Eng. C Mater. Biol. Appl. 2016, 61, 158-168. [CrossRef] [PubMed]

7. Bai, H.; Zhang, Q.; He, T.; Zheng, G.; Zhang, G.; Zheng, L.; Ma, S. Adsorption dynamics, diffusion and isotherm models of poly(NIPAM/LMSH) nanocomposite hydrogels for the removal of anionic dye amaranth from an aqueous solution. Appl. Clay Sci. 2016, 124-125, 157-166. [CrossRef]

8. JagadeeshBabu, P.E.; Suresh Kumar, R.; Maheswari, B. Synthesis and characterization of temperature sensitive P-NIPAM macro/micro hydrogels. Colloids Surf. A Physicochem. Eng. Asp. 2011, 384, 466-472. [CrossRef] 
9. Shepherd, J.; Sarker, P.; Rimmer, S.; Swanson, L.; MacNeil, S.; Douglas, I. Hyperbranched poly(NIPAM) polymers modified with antibiotics for the reduction of bacterial burden in infected human tissue engineered skin. Biomaterials 2011, 32, 258-267. [CrossRef] [PubMed]

10. Francis, R.; Baby, D.K.; Kumar, D.S. Poly(N-isopropylacrylamide) hydrogel: Effect of hydrophilicity on controlled release of ibuprofen at different pH. J. Appl. Polym. Sci. 2012, 124, 5079-5088. [CrossRef]

11. Kang, M.K.; Hong, S.K.; Kim, J.-C. Release property of microgels formed by electrostatic interaction between poly( $\mathrm{N}$-isopropylacrylamide-co-methacrylic acid) and poly( $\mathrm{N}$-isopropylacrylamide-co-dimethylamin oethylmethacrylate). J. Appl. Polym. Sci. 2012, 125, 1993-1999. [CrossRef]

12. Choi, J.-H.; Lee, H.Y.; Kim, J.-C. Release behavior of freeze-dried alginate beads containing poly(N-isopropylacrylamide) copolymers. J. Appl. Polym. Sci. 2008, 110, 117-123. [CrossRef]

13. Chen, S.; Jiang, L.; Dan, Y. Preparation and thermal response behavior of poly( $N$-isopropylacrylamide- $c 0$-a crylic acid) microgels via soap-free emulsion polymerization based on aibn initiator. J. Appl. Polym. Sci. 2011, 121, 3322-3331. [CrossRef]

14. Fernandez, V.V.A.; Tepale, N.; Sánchez-Díaz, J.C.; Mendizábal, E.; Puig, J.E.; Soltero, J.F.A. Thermoresponsive nanostructured poly( $N$-isopropylacrylamide) hydrogels made via inverse microemulsion polymerization. Coll. Polym. Sci. 2005, 284, 387-395. [CrossRef]

15. Gonçalves, M.A.D.; Pinto, V.D.; Costa, R.A.S.; Dias, R.C.S.; Hernándes-Ortiz, J.C.; Costa, M.R.P.F.N. Stimuli-responsive hydrogels synthesis using free radical and RAFT polymerization. Macromol. Symp. 2013, 333, 41-54. [CrossRef]

16. Gan, D.; Lyon, A.L. Tunable swelling kinetics in core-shell hydrogel nanoparticles. J. Am. Chem. Soc. 2001, 123, 7511-7517. [CrossRef] [PubMed]

17. Li, P.; Kim, N.H.; Hui, D.; Rhee, K.Y.; Lee, J.H. Improved mechanical and swelling behavior of the composite hydrogels prepared by ionic monomer and acid-activated Laponite. Appl. Clay Sci. 2009, 46, 414-417. [CrossRef]

18. Li, L.; Du, X.; Deng, J.; Yang, W. Synthesis of optically active macroporous poly( $N$-isopropylacrylamide) hydrogels with helical poly( $N$-propargylamide) for chiral recognition of amino acids. React. Funct. Polym. 2011, 71, 972-979. [CrossRef]

19. Rennerfeldt, D.A.; Renth, A.N.; Talata, Z.; Gehrke, S.H.; Detamore, M.S. Tuning mechanical performance of poly(ethylene glycol) and agarose interpenetrating network hydrogels for cartilage tissue engineering. Biomaterials 2013, 34, 8241-8257. [CrossRef] [PubMed]

20. Baek, K.; Clay, N.E.; Qin, E.C.; Sullivan, K.M.; Kim, D.H.; Kong, H. In situ assembly of the collagen-polyacrylamide interpenetrating network hydrogel: Enabling decoupled control of stiffness and degree of swelling. Eur. Polym. J. 2015, 72, 413-422. [CrossRef]

21. Chinnam, P.R.; Mantravadi, R.; Jimenez, J.C.; Dikin, D.A.; Wunder, S.L. Lamellar, micro-phase separated blends of methyl cellulose and dendritic polyethylene glycol, POSS-PEG. Carbohydr. Polym. 2016, 136, 19-29. [CrossRef] [PubMed]

22. Nag, S.; Sachan, A.; Castro, M.; Choudhary, V.; Feller, J.F. Spray layer-by-layer assembly of POSS functionalized CNT quantum chemo-resistive sensors with tuneable selectivity and ppm resolution to VOC biomarkers. Sens. Actuators B Chem. 2016, 222, 362-373. [CrossRef]

23. Wang, D.; Fredericks, P.M.; Haddad, A.; Hill, D.J.T.; Rasoul, F.; Whittaker, A.K. Hydrolytic degradation of POSS-PEG-lactide hybrid hydrogels. Polym. Degrad. Stab. 2011, 96, 123-130. [CrossRef]

24. Wang, L.; Zeng, K.; Zheng, S. Hepta(3,3,3-trifluoropropyl) polyhedral oligomeric silsesquioxane-capped poly( $N$-isopropylacrylamide) telechelics: Synthesis and behavior of physical hydrogels. ACS Appl. Mater. Interfaces 2011, 3, 898-909. [CrossRef] [PubMed]

25. Wang, D.K.; Varanasi, S.; Strounina, E.; Hill, D.J.; Symons, A.L.; Whittaker, A.K.; Rasoul, F. Synthesis and characterization of a POSS-PEG macromonomer and POSS-PEG-PLA hydrogels for periodontal applications. Biomacromolecules 2014, 15, 666-679. [CrossRef] [PubMed]

26. Hou, X.; Yang, W.; Li, A.; Hou, J.; Zhang, C. Effects of incorporating acrylolsobutyl polyhedral oligomeric silsesquioxane on the properties of $\mathrm{p}(\mathrm{N}$-isopropylacrylamide-co-poly(ethylene glycol) diacrylate) hybrid hydrogels. Polym. Bull. 2016, 74, 1831-1847. [CrossRef]

27. Chen, K.; Zhang, Q.; Chen, B.; Chen, L. Nanocomposite hydrogels with rapid thermal-responsibility by using surfactant detergent as template. Appl. Clay Sci. 2012, 58, 114-119. [CrossRef] 
28. Zeng, K.; Fang, Y.; Zheng, S. Organic-inorganic hybrid hydrogels involving poly(N-isopropylacrylamide) and polyhedral oligomeric silsesquioxane: Preparation and rapid thermoresponsive properties. J. Polym. Sci. Part B Polym. Phys. 2009, 47, 504-516. [CrossRef]

29. Shi, Y.; Xiong, D.; Liu, Y.; Wang, N.; Zhao, X. Swelling, mechanical and friction properties of PVA/PVP hydrogels after swelling in osmotic pressure solution. Mater. Sci. Eng. C Mater. Biol. Appl. 2016, 65, 172-180. [CrossRef] [PubMed]

30. Liu, S.; Zhang, J.; Cui, X.; Guo, Y.; Zhang, X.; Hongyan, W. Synthesis of chitosan-based nanohydrogels for loading and release of 5-fluorouracil. Colloids Surf. A Physicochem. Eng. Asp. 2016, 490, 91-97. [CrossRef]

31. Blanco, M.D.; Guerrero, S.; Benito, M.; Fernández, A.; Teijón, C.; Olmo, R.; Katime, I.; Teijón, J.M. In vitro and in vivo evaluation of a folate-targeted copolymeric submicrohydrogel based on $N$-isopropylacrylamide as 5-fluorouracil delivery system. Polymers 2011, 3, 1107-1125. [CrossRef]

32. Lo, C.L.; Lin, K.M.; Hsiue, G.H. Preparation and characterization of intelligent core-shell nanoparticles based on poly(D,L-lactide)-g-poly( $N$-isopropyl acrylamide-co-methacrylic acid). J. Control. Release 2005, 104, 477-488. [CrossRef] [PubMed]

33. Oliveira, D.; Gomes, C.P.; Dias, R.C.S.; Costa, M.R.P.F.N. Molecular imprinting of 5-fluorouracil in particles with surface RAFT grafted functional brushes. React. Funct. Polym. 2016, 107, 35-45. [CrossRef]

34. Arakawa, Y.; Nakano, M.; Juni, K.; Arita, T. Physical properties of pyrimidine and purine antimetabolites. I. The effects of salts and temperature on the solubility of 5-fluorouracil, 1-(2-tetrahydrofuryl)-5-fluorouracil, 6-mercaptopurine, and thioinosine. Chem. Pharm. Bull. 1976, 24, 1654-1657. [CrossRef] [PubMed]

(c) 2018 by the authors. Licensee MDPI, Basel, Switzerland. This article is an open access article distributed under the terms and conditions of the Creative Commons Attribution (CC BY) license (http:/ / creativecommons.org/licenses/by/4.0/). 


\title{
Article \\ POSS Dental Nanocomposite Resin: Synthesis, Shrinkage, Double Bond Conversion, Hardness, and Resistance Properties
}

\author{
Yizhi Liu ${ }^{1, *}$, Xiaorong Wu ${ }^{1}$, Yi Sun ${ }^{1}$ and Weili Xie ${ }^{2}$ \\ 1 Department of Astronautic Science and Mechanics, Harbin Institute of Technology, Harbin 150001, China; \\ wxr@hit.edu.cn (X.W.); sunyi@hit.edu.cn (Y.S.) \\ 2 Department of Stomatology, Harbin Medical University, Harbin 150001, China; xwl811@126.com \\ * Corresponding authors: liuyizhi@hit.edu.cn; Tel.: +86-451-8641-8100
}

Received: 5 March 2018; Accepted: 23 March 2018; Published: 26 March 2018

\begin{abstract}
Nanocomposite dental resins with 0, 2, 5, and $10 \mathrm{wt} \%$ methacryl polyhedral oligomeric silsesquioxane (POSS) as filler in the resin matrix were prepared by a light curing method. The atomic force microscopy (AFM), fourier transform infrared spectroscopy (FTIR), nanoindentation, and nanoscratch tests were carried out to study the effect of POSS contents on the compatibility, double bond conversion, volumetric shrinkage, hardness, modulus, and resistance of the dental resins. POSS was very uniformly dispersed and showed a good compatibility with the matrix. The double bond conversion increased and the volume reduced with the addition of POSS. As the POSS addition increased, the mechanical properties increased initially. Small addition of POSS remarkably enhanced the hardness and scratch resistance of the resin matrix.
\end{abstract}

Keywords: dental resin; methacryl POSS; shrinkage; hardness; scratch resistance

\section{Introduction}

Increasingly, conventional alloys in the current dental field have been replaced by resin materials due to their advantages such as operation technique and aesthetic quality [1,2]. Nanofiller-reinforced dental composites show large advantages in large area restoration applications due to its easy processing and good biocompatibility [3,4]. In the past few years, the application of new nanofillers in dental resins to improve its mechanical property and anti-caries function has become a popular research topic [5-9].

Hybrid organic-inorganic nanocomposites, which incorporate polyhedral oligomeric silsesquioxane (POSS) into polymeric matrices including dental resins, have received a considerable amount of attention. Polyhedral oligomeric silsesquioxane (POSS) is an organic-inorganic hybrid nanomaterial. Its formula is $\left(\mathrm{RSiO}_{3 / 2}\right)_{\mathrm{n}}$, where $\mathrm{n}$ is the number of the silicon atoms of the cage (1-3 nm in size) surrounded by organic corner groups $\mathrm{R}$. There are very different types of POSS according to the various R organic groups $[10,11]$. The physical mixture can be easily used to get together or react with monomer via copolymerization or blending to improve the physical properties [12-14]. Compared with traditional fillers, POSS can significantly improve the mechanical properties $[15,16]$ of the composite and overcome the disadvantage of some polymer matrix materials, such as poor wear resistance, defects, and processing difficulties. At the same time, even the addition of a very small amount of POSS enhances the thermal [17-20], anti-oxygen erosion [21-23], and self-assembling [24-27] properties. Furthermore, the typical $\mathrm{Si}-\mathrm{O}$ bonds and organic groups of POSS lead to evident improvements of the marginal adaptation of the matrix, ensuring good biocompatibility and comfort level [28], as well as remarkably reducing micro effusion risk and secondary caries $[5,29]$. In this study, colorless oil methacryl POSS (Figure 1) was chosen to be incorporated into neat resin due to its good compatibility. 


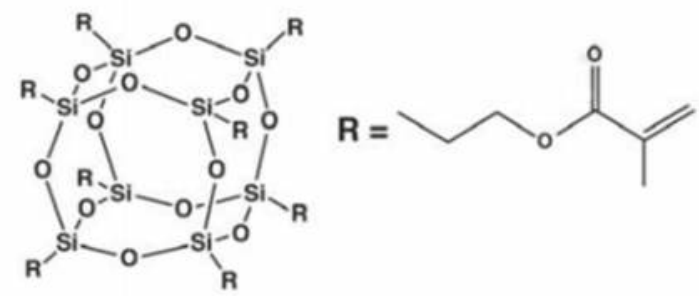

Figure 1. The molecule structure of methacryl polyhedral oligomeric silsesquioxane (POSS).

Researchers have been making efforts to study the morphology, shrinkage, and physical properties of composite resins in the dental field and the aim is to focus on the synthesis of novel dental resin composites with low shrinkage and high mechanical properties. Hao Fong et al. [30] analyzed the following percentages $(0,2 \%, 5 \%, 10 \%, 25 \%$, and $50 \%$ ) of methacryl POSS (POSS-MA) in Bisphenol A glycerolate dimethacrylate (Bis-GMA), which work partially as novel dental restorative composites by the curing method. For the measurement of volumetric shrinkage, the curing time was set to be $1 \mathrm{~min}$. After 1 hour post curing time, the light was turned on for another $30 \mathrm{~s}$. His work indicated that Bis-GMA with POSS-MA did not affect the volumetric shrinkage, and the mechanical property of the composite was optimized only with a small percentage of POSS-MA substitution. Kleverlaan and Feilzer [31] evaluated the shrinkage, contraction stress, and tensile modulus of various commercially available dental resin composites. The shrinkage and contraction stress properties were enhanced by using pre-polymerized clusters. In our studies [32,33], novel dental nanocomposites with BG (barium oxide glass powder) and nano $\mathrm{SiO}_{2}$ fillers with different contents of methacryl POSS were developed, respectively. The polymerization shrinkage and mechanical properties were analyzed. To the best of our knowledge, there are not any reports about dental resin incorporated with the relatively low addition of POSS. But it seems that a low addition of POSS as nanofiller evidently enhances the performances of matrix [34,35].

In this study, dental resin composites with various amounts of POSS were processed using the light curing method. The influence of a low addition of POSS to dental resin was analyzed. The atomic force microscopy (AFM), FTIR, nanoindentation, and nanoscratch tests were performed, and the effects of the contents of POSS on the compatibility, double bond conversion, volumetric shrinkage, hardness, and resistance were studied.

\section{Experimental}

\subsection{Materials}

Bisphenol A glycerolate dimethacrylate (Bis-GMA) and Tri(ethylenglycol) dimethacrylate (TEGDMA) $98 \%$ were purchased from Aldrich Chemical Co. China (Shanghai, China). Camphorquinone (CQ, 97\%) was used as a visible light photo-initiator and was selected with its co-initiator 2-(dimethylamino) ethylmethacrylate (DMAEMA, 98\%) for this research. The nano filler, methacryl POSS, was purchased from Hybrid Plastics (Hattiesburg, MS, USA).

\subsection{Preparation of POSS Composite Resins}

A resin matrix solution containing Bis-GMA, TEGDMA, CQ, and DMAEMA was prepared by mixing in a light resistance environment after sufficient mixing. Then different percentages methacryl POSS, according to the quantities reported in Table 1, were added and blended uniformly by a magnetic stir bar. 
Table 1. The compositions proportion of POSS hybrid dental nanocomposites.

\begin{tabular}{cccccc}
\hline & \multicolumn{4}{c}{ Resin Matrix } & Nanofiller \\
\cline { 2 - 6 } & \multicolumn{2}{c}{ Resin System } & Light Photo-Initiator & \\
\cline { 2 - 6 } & Bis-GMA & TEGDMA & CQ & DMAEMA & methacryl POSS \\
\hline P00 & 49.5 & 49.5 & 0.5 & 0.5 & 0 \\
P02 & 47.5 & 49.5 & 0.5 & 0.5 & 2 \\
P05 & 44.5 & 49.5 & 0.5 & 0.5 & 5 \\
P10 & 39.5 & 49.5 & 0.5 & 0.5 & 10 \\
\hline
\end{tabular}

The whole process should be performed under vacuum conditions. The mixtures went through a light curing process which lasted for $40 \mathrm{~s}$ at room temperature in stainless steel molds. After being soaked in distilled water at $37^{\circ} \mathrm{C}$ for $24 \mathrm{~h}$, they were placed in configured artificial saliva (according to ISOTR1021) at $36.5^{\circ} \mathrm{C}$ for 4 more weeks. Finally, the dimensions of the specimens were measured accurately before testing.

\section{Characterization}

\subsection{AFM Characterization}

AFM topography images were obtained in Peak Force Tapping on the Bruker instruments (Bruker, Billerica, MA, USA). The scan rate was set to be $1 \mathrm{~Hz}$ and imaging was carried out on Multimode 8 .

\subsection{FTIR Characterization and Measurement of Double Bond Conversion}

FTIR spectrometer (Avatar360, Nicolet, Madison, WI, USA) was used to carry out the Fourier transform infrared (FTIR) spectroscopy analysis. The scan range was from 4000 to $400 \mathrm{~cm}^{-1}$, with a resolution of $4 \mathrm{~cm}^{-1}$.

The degree of double bond conversion (DC) was monitored by FTIR. The DC was calculated from the methacrylate $\mathrm{C}=\mathrm{C}$ peak at $1636 \mathrm{~cm}^{-1}$ and normalized against the carbonyl $\mathrm{C}=\mathrm{O}$ peak at $1720 \mathrm{~cm}^{-1}$ according to the Equation 1.

$$
D C(t)=\frac{\left(\frac{A_{C=C}}{A_{C=O}}\right)_{0}-\left(\frac{A_{C=C}}{A_{C=O}}\right)_{t}}{\left(\frac{A_{C=C}}{A_{C=O}}\right)_{0}}
$$

where $A_{C=C}$ and $A_{C=O}$ are the absorbance peak areas at 1636 and $1720 \mathrm{~cm}^{-1}$, which are characteristic of methacrylate $\mathrm{C}=\mathrm{C}$ and carbonyl, respectively. $\left(\frac{A_{C=C}}{A_{C=O}}\right)_{0}$ and $\left(\frac{A_{C=C}}{A_{C=O}}\right)_{t}$ were the initial and terminal ratio of these two absorbance peak areas.

\subsection{Shrinkage}

The densities of both uncured and cured resin samples were measured by a pycnometer to determine the polymerization shrinkage according to the Archimedes' principle. The volumetric shrinkage was calculated using the following equation:

$$
\Delta V \%=\left(1-\rho_{\text {uncured }} / \rho_{\text {cured }}\right) \times 100 \%
$$

where $\rho_{\text {uncured }}$ and $\rho_{\text {cured }}$ are the density of uncured and cured resin specimens, respectively. They are calculated according to the following equations:

$$
\begin{gathered}
\rho_{\text {uncured }}=\frac{m_{2}-m_{0}}{m_{1}-m_{0}} \times \rho_{w} \\
\rho_{\text {cured }}=\frac{m_{s}}{m_{1}-m_{3}} \times \rho_{w}
\end{gathered}
$$


where $m_{0}$ is the mass of the empty bottle. Here, $\mathrm{m}_{1}$ is the mass of the bottle full of water at $20^{\circ} \mathrm{C} ; \mathrm{m}_{2}$ is the mass of the bottle full of solution at $20^{\circ} \mathrm{C} ; \mathrm{m}_{3}$ is the mass of the bottle with specimen and water; $m_{\mathrm{S}}$ is the mass of the specimen; and $\rho_{w}$ is the density of water.

\subsection{Nanoindentation Testing}

According to the Oliver-Pharr method [36,37], a nanoindentation test using G200 Nano Indenter (Agilent Technologies, Santa Clara, CA, USA) was used to evaluated the hardness of the nanocomposite resins. The tip of the diamond triangular pyramid Berkovich indenter (TB 20114 ISO) had a radius of about $20 \mathrm{~nm}$. The maximum penetration depth during the indentation test was $2000 \mathrm{~nm}$ for each sample. A constant strain rate of $0.05 \mathrm{~s}^{-1}$ was ensured by the loading speed. The results of 8 samples were averaged to get the most accurate results.

\subsection{Nanoscratch Testing}

The resistance of the POSS-reinforced resin was evaluated by nanoscratch testing using a Nano Indenter G200 instrument (Agilent Technologies, USA). The method employed was the "G-Series single direction wear test". The authors followed a test procedure similar as published elsewhere $[38,39]$. The same experimental environment and allowable temperature drift rate were used as in the nanoindentation test. The original topography and surface roughness of the sample were measured by pre-scanning the sample surface. The pre-scanning was carried out on $20 \%$ of the scratch length during the scratch test with a relatively light load of $50 \mu \mathrm{N}$. The load value during the scratch test was $200 \mathrm{mN}$ and the load was applied on the indenter tip. The scratch length during the test was $400 \mu \mathrm{m}$ and the speed was $10 \mu \mathrm{m} / \mathrm{s}$. The scratch depth under the increasing scratch load could be obtained from the difference values by profiling the surface, and a total length of about $560 \mu \mathrm{m}$ for each test was generated (including $80-\mu \mathrm{m}$ rescanning and post-scanning at the two ends of the real scratch path), whereas the real effective scratch length was $400 \mu \mathrm{m}$, as applied to all specimens. Thus, the effective penetration depths, i.e., the scratch depth and the residual depth during and after the scratch test at different scratch distances, could be obtained. Three independent scratch tests were carried out for each sample and the results were averaged.

\section{Results and Discussion}

It was found during the tests that a higher percentage of the nano filler resulted in the deterioration of the transparency. However, this led to a high viscosity of the sample. Deterioration in the transparency thus led to a less light radiation fluence and a lower curing level of composites.

\subsection{AFM}

The morphologies of a composite with the size of $2 \mu \mathrm{m} \times 2 \mu \mathrm{m}$, containing $0,2,5$, and $10 \mathrm{wt} \%$ POSS, are shown in the AFM images in Figure 2, respectively. 

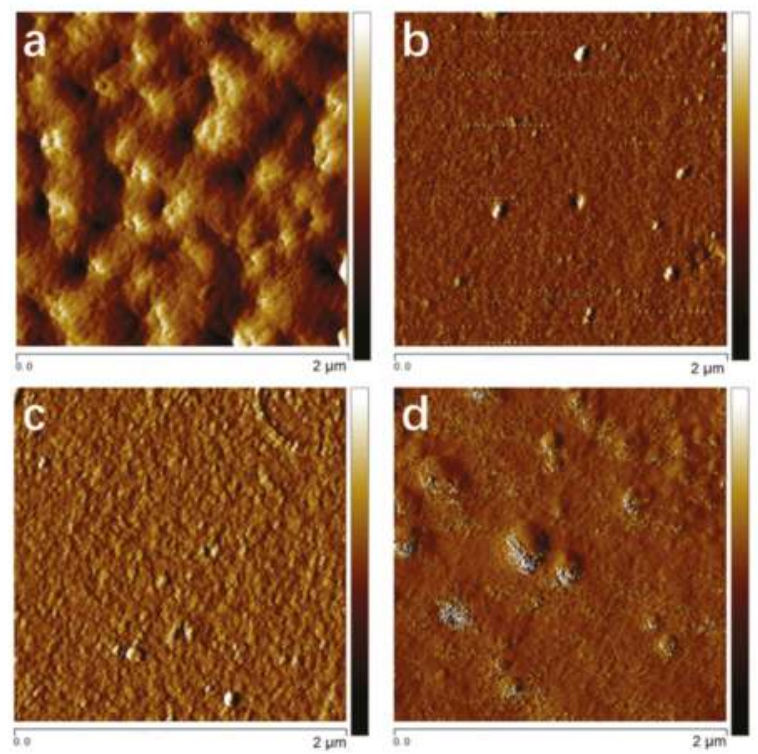

Figure 2. The atomic force microscopy (AFM) images of the structure of composite resins with different POSS additions: (a) P00, (b) P02, (c) P05, (d) P10.

POSS showed a good compatibility with the matrix and could be uniformly dispersed at a low percentage of addition. However, once the POSS content reached $10 \mathrm{wt} \%$, the aggregation of nanoparticles can be observed in the composite resin due to the self-assembly of POSS [40]. When the contents of POSS nanoparticles were low, the cavity and high surface roughness of the resin declined at the surface level, and the surface flatness and the porosity reduced. Due the viscous property of the POSS used in this work, as well as the long organic R chains around POSS which make the resin matrix molecules to distribute uniformly, the surfaces of all POSS composite resins are smoother than the one of the neat resin at the nano-size level in roughness. The low addition of POSS improves the surface smoothness.

\subsection{FTIR Analysis and Double Bond Conversion (DC)}

The FTIR spectra of composite resins with different POSS contents after curing are shown in Figure 3. The band at $1635 \mathrm{~cm}^{-1}$ was attributed to the characteristic absorption peaks of the $C=C$ bond. When the curing happened, the methacrylate double bonds partly converted and the resins polymerized [41]. The intensity of $\mathrm{C}=\mathrm{C}$ absorption at $1635 \mathrm{~cm}^{-1}$ became evidently weaker.

The values of double bond conversion of composite resins with different POSS contents are shown in Figure 4. In this study, the curing process at room temperature took $40 \mathrm{~s}$ for each sample. The double bond conversion of the resin matrix was $42.1 \%$. The double bond conversion of the POSS nanocomposite resin reached up to $55 \%$. The variation trend is not obvious with the increasing addition even to $10 \mathrm{wt} \%$. It can be explained by the fact that the uniformly distributed POSS in the polymer matrix at the nano-level allows the resin matrix to react more easily, which obviously increased the curing effect [42]. 


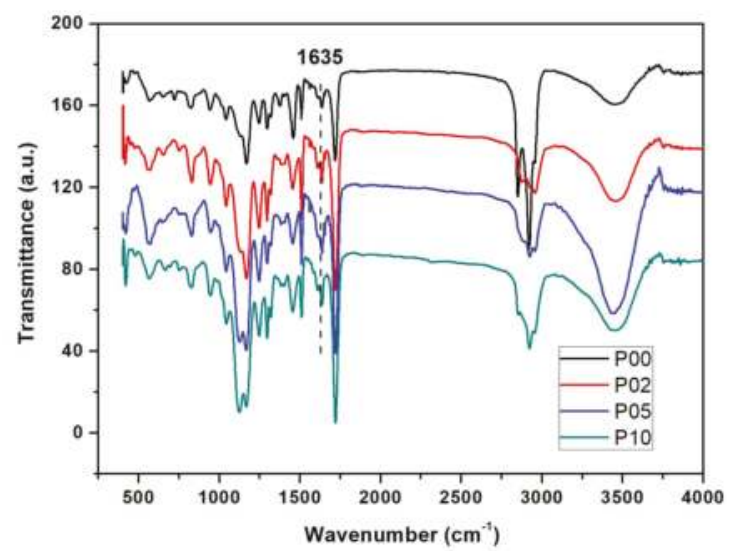

Figure 3. FTIR spectra of composite resins with different POSS additions.

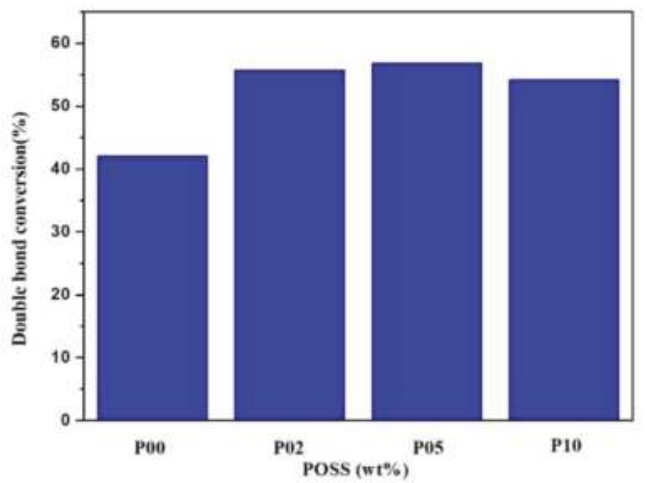

Figure 4. Double bond conversion of composite resins with different POSS additions.

\subsection{Volumetric Shrinkage}

In this subsection, the shrinkage of composite resins synthesized were studied. The value of volumetric shrinkage of the resin matrix was $10.65 \%$. As the percentage of POSS increased, the volume shrinkage of composites became lower. The values were about $9.07 \%, 9.85 \%$, and $7.81 \%$ for the additions of $2 \%, 5 \%$, and $10 \%$, respectively.

In our previous work [43], the densities of uncured and cured models were calculated and the volume shrinkages of resins with different weight percentages of POSS were estimated. Compared with the results of the previous work, the values were almost the same. The results from other studies in the literatures also showed that the shrinkage values of typical resins of dental methacrylate-based monomers were between $6 \%$ and $10 \%$ [31,44,45]. Before the curing process, large chain monomers such as Bis-GMA and TEGDMA were loosely dispersed in the matrix. After curing, the reaction with monomers occurred. The movement of chains was not combined by amounts of cross-linked points and the free volume of resins changed remarkably. This results in a remarkable decrease of the shrinkage of resins. POSS has a three-dimensional organic-inorganic cage structure and its volume is much larger than those of Bis-GMA and TEGDMA. When POSS is incorporated in resins, it can attract and even entwine monomer chains. The monomers can disperse more tightly. POSS limits the change of the free volume of matrix during the reaction and the volume of the nanocage of POSS itself does not change after the reaction. In brief, the volume shrinkage of POSS dental composites reduces. 
Besides, our previous experimental research and the study of Fong also investigated the volume shrinkage of the composite resins with an additional 60 and $70 \mathrm{wt} \%$ of BG powder [30,33]. The values of the shrinkage of POSS composites resin with a large percentage of additional BG powder were lower than the results reported in this work (without inorganic powder). The reasons are because the incorporation of a percentage of inorganic filler, BG, was very high and the volume of it did not change after the reaction. Consequently, the volumetric shrinkage of resins was very low. Even though, the trend was similar with the results of our previous research.

\subsection{Hardness and Elastic Modulus}

The hardness and resistance are two of the most important properties for dental restorations. In this study, the resistance of the POSS dental composites was evaluated by the scratch depth. The scratch depth can be used to evaluate the deformation resistance and hardness of the samples at a specific load [46,47]. The hardness, elastic modulus, and scratch depth of the nanocomposite resin were obtained by nanotesting technology. It should be emphasized that the sample was made of organic resin without traditional inorganic filler. Before the testing, the samples were placed in the configured artificial saliva at $36.5^{\circ} \mathrm{C}$ for 4 weeks.

The testing results of the hardness, elastic modulus, and the average scratch depth are listed in Table 2. The influence of composite resins with different POSS contents on the scratch profiles is shown in Figure 5. A total length of about $560 \mu \mathrm{m}$ for each test was generated (including $80-\mu \mathrm{m}$ pre-scanning and post-scanning at the two ends of real scratch path). The scratch region was from 80 to $480 \mu \mathrm{m}$ in the scratch distance, in which the profile was essentially affected by the load value, hardness, and elasticity, revealing the scratch resistance property of a sample.

Table 2. The characterization of the hardness, elastic modulus, and average scratch depth of samples.

\begin{tabular}{cccc}
\hline Samples & Hardness (GPa) & Elastic Modulus (GPa) & Average Scratch Depth (nm) \\
\hline P00 & $0.204 \pm 0.008$ & $2.931 \pm 0.163$ & $-19,111 \pm 502$ \\
P02 & $0.243 \pm 0.013$ & $3.388 \pm 0.213$ & $-15,684 \pm 556$ \\
P05 & $0.322 \pm 0.019$ & $4.136 \pm 0.204$ & $-12,349 \pm 473$ \\
P10 & $0.285 \pm 0.017$ & $3.491 \pm 0.241$ & $-18,331 \pm 698$ \\
\hline
\end{tabular}

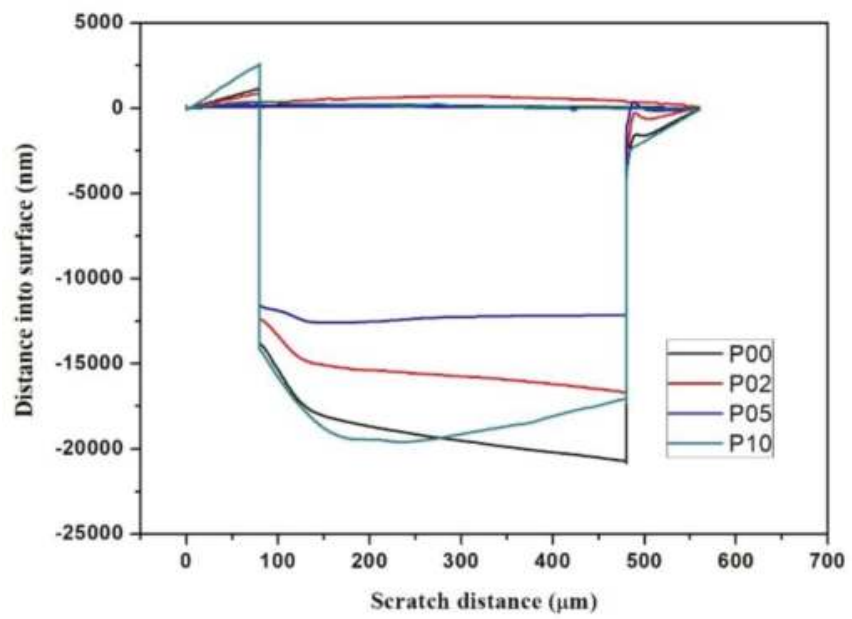

Figure 5. Influence of composite resins with different POSS additions on the scratch profiles. 
As the POSS addition increased, the mechanical properties increased initially. Once it exceeded $5 \mathrm{wt} \%$, the mechanical properties fell down. A small addition of POSS remarkably enhanced the hardness and the scratch resistance of the resin matrix. These properties showed the similar variation trend according to the percentage of POSS, which indicated that it had the same influence on the composites' matrix. POSS, serving as a nucleating agent, attracts matrix chains, which increases the rigidity of the systems and enhances the mechanical properties. While a larger content of POSS perhaps results in a large reduction of the crosslinks among the matrix chains owing to the aggregation of POSS, it enables the polymer matrix to form plastic flow easily and reduces the mechanical properties $[12,42,48]$. The novel dental composite with $5 \mathrm{wt} \%$ POSS addition possesses superior performances in the relative increments of hardness, elastic modulus, and scratch depth.

Compared with $\mathrm{SiO}_{2}$, POSS is a unique kind of filler with a hard inorganic Si-O cage core surrounded by soft organic chains. The overall performance of POSS derives from the combined action of hard "core" and soft "shell" [49]. When the addition is small, POSS, as a nucleating agent, attracts polymer chains and forms more condensed particles. POSS can increase the bonding energy of the resin matrix and enhance the mechanical properties [50,51]. If the composites have good dispersion, the strong stress transfer is efficient $[52,53]$. The interface adhesion of the matrix and the filler is strengthened and most loads are carried by the hard core of POSS; as a result, the mechanical properties increase. When the addition of the filler is high, on one hand, large aggregation occurs. On the other hand, the distance between the filler is reduced and the stress transfer at the filler and matrix interface is inefficient. The R group of POSS twines together and the constraint level enhances, which results in an increasing thickness of the soft shell. The ability of the hard inorganic Si-O cage to act as an enhanced agent reduces, and the one of soft organic R groups increases [54,55].

Compared with the values of the hardness in our previous work [33], it is found the hardness values achieved in the current research were lower. This is because that some kinds of inorganic fillers are not added into the dental composite resins at high contents. In clinical applications, we find that although the hardness increases with the high addition of inorganic filler, some other key properties of dental resins, such compressive strength, become low after molding. As a result, the bulk of composite resins generates cracks easily and portions of it eventually turn into small particles due to frequent chewing. It can produce some problems in clinical applications when a large addition inorganic filler with a big particle size is employed. Certainly, the physical properties of dental products need to be as close as possible to those of dentin. So, it seems an excellent choice to base the preparation of dental restoration materials on organic resins only. Such organic resins derived from monomers can be prepared by polymerization or incorporated with only a small addition of inorganic filler.

\section{Conclusions}

In this paper, POSS is shown to be very compatible with the matrix. Weight fractions of $2 \%, 5 \%$, and $10 \%$ of POSS in the resin matrix lead to an increase of the double bond conversion to about $55 \%$ when the curing time of each sample is $40 \mathrm{~s}$. The values of volumetric shrinkage are about $9.07 \%, 9.85 \%$, and $7.81 \%$, respectively, at the above weight fractions. POSS limits the change in the free volume of the matrix during the reaction, but the volume of the nanocage of POSS itself does not change after the reaction. Thus, the shrinkage of resins with incorporated POSS decreases.

As the content of POSS increases, the mechanical properties increase initially. The addition of a small amount of POSS remarkably enhances the hardness and the scratch resistance of the resin matrix. Once it exceeds $5 \mathrm{wt} \%$, the mechanical properties deteriorate. POSS, serving as a nucleating agent, attracts matrix chains and thus increases the rigidity of the systems and enhances the mechanical properties. However, a larger content of POSS may result in a large reduction of the crosslinks among matrix chains owing to the aggregation of POSS.

Acknowledgments: The authors thank Xinran Zhou (School of Materials Science and Engineering, Nanyang Technological University, Singapore) for helpful discussions. The authors thank the National Natural Science Foundation of China $(11302059,11602073)$ for the financial support of this research, and the research is also 
supported by "the Fundamental Research Funds for the Central Universities" (Grant No.HIT.NSRIF.201821) and the State Scholarship Fund (201706125076) from China Scholarship Council.

Author Contributions: Yi Sun and Weili Xie conceived and designed the experiments; Yizhi Liu and Xiaorong Wu performed most of the experiments, analyzed the data and plotted the figures; Yizhi Liu wrote the paper and provided revise the manuscript.

Conflicts of Interest: The authors declare no conflict of interest.

\section{References}

1. Buonocore, M.G. A simple method of increasing the adhesion of acrylic filling materials to enamel surfaces. J. Dent. Res. 1955, 34, 849-853. [CrossRef] [PubMed]

2. Van Landuyt, K.L.; Snauwaert, J.; De Munck, J.; Peumans, M.; Yoshida, Y.; Poitevin, A.; Coutinho, E.; Suzuki, K.; Lambrechts, P.; Van Meerbeek, B. Systematic review of the chemical composition of contemporary dental adhesives. Biomaterials 2007, 28, 3757-3785. [CrossRef] [PubMed]

3. Van Nieuwenhuysen, J.P.; D'Hoore, W.; Carvalho, J.; Qvist, V. Long-term evaluation of extensive restorations in permanent teeth. J. Dent. 2003, 31, 395-405. [CrossRef]

4. Miao, X.; Li, Y.; Zhang, Q.; Zhu, M.; Wang, H. Low shrinkage light curable dental nanocomposites using $\mathrm{SiO} 2$ microspheres as fillers. Mater. Sci. Eng. C 2012, 32, 2115-2121. [CrossRef]

5. Yudovin-Farber, I.; Beyth, N.; Nyska, A.; Weiss, E.I.; Golenser, J.; Domb, A.J. Surface characterization and biocompatibility of restorative resin containing nanoparticles. Biomacromolecules 2008, 9, 3044-3055. [CrossRef] [PubMed]

6. Liu, Y.; Tan, Y.; Lei, T.; Xiang, Q.; Han, Y.; Huang, B. Effect of porous glass-ceramic fillers on mechanical properties of light-cured dental resin composites. Dent. Mater. 2009, 25, 709-715. [CrossRef] [PubMed]

7. Moharamzadeh, K.; Van Noort, R.; Brook, I.; Scutt, A. HPLC analysis of components released from dental composites with different resin compositions using different extraction media. J. Mater. Sci. 2007, 18, 133-137. [CrossRef] [PubMed]

8. Xu, H.H.; Moreau, J.L.; Sun, L.; Chow, L.C. Strength and fluoride release characteristics of a calcium fluoride based dental nanocomposite. Biomaterials 2008, 29, 4261-4267. [CrossRef] [PubMed]

9. Tanimoto, Y.; Nemoto, K. Influence of particle size of fillers on frictional wear of dental composite resins. Compos. Interfaces 2004, 11, 15-24. [CrossRef]

10. Blanco, I.; Abate, L.; Bottino, F.A.; Bottino, P. Synthesis, characterization and thermal stability of new dumbbell-shaped isobutyl-substituted POSSs linked by aromatic bridges. J. Therm. Anal. Calorim. 2014, 117, 243-250. [CrossRef]

11. Blanco, I.; Bottino, F.; Abate, L. Influence of n-alkyl substituents on the thermal behaviour of Polyhedral Oligomeric Silsesquioxanes (POSSs) with different cage's periphery. Thermochim. Acta 2016, 623, 50-57. [CrossRef]

12. Liu, Y.; Sun, Y.; Zeng, F.; Liu, J.; Ge, J. Effect of POSS nanofiller on structure, thermal and mechanical properties of PVDF matrix. J. Nanopart. Res. 2013, 15. [CrossRef]

13. Ayandele, E.; Sarker, B.; Alexandridis, P. Polyhedral Oligomeric Silsesquioxane (POSS)-Containing Polymer Nanocomposites. Nanomaterials 2012, 2, 445-475. [CrossRef] [PubMed]

14. Blanco, I.; Bottino, F.A.; Cicala, G.; Latteri, A.; Recca, A. Synthesis and characterization of differently substituted phenyl hepta isobutyl-polyhedral oligomeric silsesquioxane/polystyrene nanocomposites. Polym. Compos. 2014, 35, 151-157. [CrossRef]

15. Dodiuk-Kenig, H.; Maoz, Y.; Lizenboim, K.; Eppelbaum, I.; Zalsman, B.; Kenig, S. The effect of grafted caged silica (polyhedral oligomeric silesquioxanes) on the properties of dental composites and adhesives. J. Adhes. Sci. Technol. 2006, 20, 1401-1412. [CrossRef]

16. Liu, Y.; Sun, Y.; Zeng, F.; Chen, Y.; Li, Q.; Yu, B.; Liu, W. Morphology, crystallization, thermal, and mechanical properties of poly(vinylidene fluoride) films filled with different concentrations of polyhedral oligomeric silsesquioxane. Polym. Eng. Sci. 2012. [CrossRef]

17. Xu, Z.; Zhao, Y.; Wang, X.; Lin, T. A thermally healable polyhedral oligomeric silsesquioxane (POSS) nanocomposite based on Diels-Alder chemistry. Chem. Commun. 2013, 49, 6755-6757. [CrossRef] [PubMed]

18. Huang, Y.; Gong, S.-D.; Huang, R.; Cao, H.-J.; Lin, Y.-H.; Yang, M.; Li, X. Polyhedral oligomeric silsesquioxane containing gel polymer electrolyte based on a PMMA matrix. RSC Advances 2015, 5, 4598-45918. [CrossRef] 
19. Fina, A.; Tabuani, D.; Carniato, F.; Frache, A.; Boccaleri, E.; Camino, G. Polyhedral oligomeric silsesquioxanes (POSS) thermal degradation. Thermochim. Acta 2006, 440, 36-42. [CrossRef]

20. Blanco, I.; Bottino, F.A. Effect of the substituents on the thermal stability of hepta cyclopentyl, phenyl substitued-Polyhedral oligomeric silsesquioxane (hcp-POSS)/polystyrene (PS) nanocomposites. AIP Conf. Proc. 2012, 1459, 247-249.

21. Minton, T.K.; Wright, M.E.; Tomczak, S.J.; Marquez, S.A.; Shen, L.; Brunsvold, A.L.; Cooper, R.; Zhang, J.; Vij, V.; Guenthner, A.J.; et al. Atomic Oxygen Effects on POSS Polyimides in Low Earth Orbit. ACS Appl. Mater. Interfaces 2011, 4, 492-502. [CrossRef] [PubMed]

22. Liu, Y.Z.; Sun, Y.; Zeng, F.L.; Zhang, Q.H.; Geng, L. Characterization and analysis on atomic oxygen resistance of POSS/PVDF composites. Appl. Surf. Sci. 2014, 320, 908-913. [CrossRef]

23. Zeng, F.; Peng, C.; Liu, Y.; Qu, J. Reactive Molecular Dynamics Simulations on the Disintegration of PVDF, FP-POSS and Their Composite during Atomic Oxygen Impact. J. Phys. Chem. A 2015. [CrossRef] [PubMed]

24. Wu, Y.C.; Kuo, S.W. Self-assembly supramolecular structure through complementary multiple hydrogen bonding of heteronucleobase-multifunctionalized polyhedral oligomeric silsesquioxane (POSS) complexes. J. Mater. Chem. 2012, 22, 2982-2991. [CrossRef]

25. Lin, Y.C.; Kuo, S.W. Hierarchical self-assembly structures of POSS-containing polypeptide block copolymers synthesized using a combination of ATRP, ROP and click chemistry. Polym. Chem. 2012, 3, 882-891. [CrossRef]

26. Kuo, S.W.; Chang, F.C. POSS related polymer nanocomposites. Prog. Polym. Sci. 2011, 36, 1649-1696. [CrossRef]

27. Blanco, I.; Bottino, F.A.; Cicala, G.; Cozzo, G.; Latteri, A.; Recca, A. Synthesis and thermal characterization of new dumbbell shaped POSS/PS nanocomposites: Influence of the symmetrical structure of the nanoparticles on the dispersion/aggregation in the polymer matrix. Polym. Compos. 2015, 36, 1394-1400. [CrossRef]

28. Siang Soh, M.; Sellinger, A.; Uj Yap, A. Dental nanocomposites. Curr. Nanosci. 2006, 2, 373-381. [CrossRef]

29. Eick, J.D.; Smith, R.E.; Pinzino, C.S. Stability of silorane dental monomers in aqueous systems. J. Dent. 2006, 34, 405-410.

30. Fong, H.; Dickens, S.H.; Flaim, G.M. Evaluation of dental restorative composites containing polyhedral oligomeric silsesquioxane methacrylate. Dent. Mater. 2005, 21, 520-529. [CrossRef] [PubMed]

31. Kleverlaan, C.J.; Feilzer, A.J. Polymerization shrinkage and contraction stress of dental resin composites. Dent. Mater. 2005, 21, 1150-1157. [CrossRef] [PubMed]

32. Liu, Y.; Sun, Y.; Zeng, F.; Xie, W.; Liu, Y.; Geng, L. Effect of nano $\mathrm{SiO}_{2}$ particles on the morphology and mechanical properties of POSS nanocomposite dental resins. J. Nanopart. Res. 2014, 16, 1-8. [CrossRef]

33. Wu, X.; Sun, Y.; Xie, W.; Liu, Y.; Song, X. Development of novel dental nanocomposites reinforced with polyhedral oligomeric silsesquioxane (POSS). Dent. Mater. 2010, 26, 456-462. [CrossRef] [PubMed]

34. Markovic, E.; Clarke, S.; Matisons, J.; Simon, G.P. Synthesis of POSS-methyl methacrylate-based cross-linked hybrid materials. Macromolecules 2008, 41, 1685-1692. [CrossRef]

35. Sanchez-Soto, M.; Schiraldi, D.A.; Illescas, S. Study of the morphology and properties of melt-mixed polycarbonate-POSS nanocomposites. Eur. Polym. J. 2009, 45, 341-352. [CrossRef]

36. Oliver, W.C.; Pharr, G.M. An Improved technique for determining hardness and elastic modulus using load and displacement sensing indentation experiments. J. Mater. Res. 1992, 7, 1564-1583. [CrossRef]

37. Oliver, W.C.; Pharr, G.M. Measurement of hardness and elastic modulus by instrumented indentation: Advances in understanding and refinements to methodology. J. Mater. Res. 2004, 19, 3-20. [CrossRef]

38. Xingwen, Z.; Lijiang, H.; Dezhi, S. Nanoindentation and nanoscratch profiles of hybrid films based on ( $\gamma$-methacrylpropyl)trimethoxysilane and tetraethoxysilane. Acta Mater. 2006, 54, 5469-5475.

39. Zeng, F.; Liu, Y.; Sun, Y.; Hu, E.; Zhou, Y. Nanoindentation, nanoscratch, and nanotensile testing of poly(vinylidene fluoride)-polyhedral oligomeric silsesquioxane nanocomposites. J. Polym. Sci. Part B Polym. Phys. 2012, 50, 1597-1611. [CrossRef]

40. Blanco, I.; Bottino, F.A.; Cicala, G.; Latteri, A.; Recca, A. A kinetic study of the thermal and thermal oxidative degradations of new bridged POSS/PS nanocomposites. Polym. Degrad. Stab. 2013, 98, 2564-2570. [CrossRef]

41. Stansbury, J.; Dickens, S.H. Determination of double bond conversion in dental resins by near infrared spectroscopy. Dent. Mater. 2001, 17, 71-79. [CrossRef]

42. Schiraldi, D.A.; Iyer, S. Role of specific interactions and solubility in the reinforcement of bisphenol A polymers with polyhedral oligomeric silsesquioxanes. Macromolecules 2007, 40, 4942-4952. 
43. Song, X.; Sun, Y.; Wu, X.; Zeng, F. Molecular dynamics simulation of a novel kind of polymer composite incorporated with polyhedral oligomeric silsesquioxane (POSS). Comput. Mater. Sci. 2011, 50, 3282-3289. [CrossRef]

44. Dupasquier, F.; Gritsch, K.; Farlay, D.; Zydowicz, N.; Grosgogeat, B. Mechanical properties and polymerization shrinkage of dental nanohybrid composites. Eur. Cells Mater. 2007, 13, 21.

45. Chen, M.H.; Chen, C.R.; Hsu, S.H.; Sun, S.P.; Su, W.F. Low shrinkage light curable nanocomposite for dental restorative material. Dent. Mater. 2006, 22, 138-145. [CrossRef] [PubMed]

46. Sanchez, J.; El-Mansy, S.; Sun, B.; Scherban, T.; Fang, N.; Pantuso, D.; Ford, W.; Elizalde, M.; MartınezEsnaola, J.; Martın-Meizoso, A. Cross Sectional nanoindentation: A new technique for thin film interfacial adhesion characterization. Acta Mater. 1999, 47, 4405-4413. [CrossRef]

47. Kim, B.; Ko, M. The assessment of the fracture behavior in spin-on organosilicates by nanoindentation and nanoscratch tests. Thin Solid Films 2009, 517, 3216-3221. [CrossRef]

48. Liu, Y.; Sun, Y.; Yu, J.; Zeng, F.; Xiao, Y.; Geng, L.; Lv, P. Study on different behaviour of poly(vinylidene fluoride)-polyhedral oligomeric silsesquioxane nanocomposites in mechanical properties and nano-tensile testing. Mater. Res. Innov. 2016, 20, 475-478. [CrossRef]

49. Baldi, F.; Bignotti, F.; Fina, A.; Tabuani, D.; Riccò, T. Mechanical characterization of polyhedral oligomeric silsesquioxane/polypropylene blends. J. Appl. Polym. Sci. 2007, 105, 935-943. [CrossRef]

50. Martins, J.N.; Bassani, T.S.; Oliveira, R.V.B. Morphological, viscoelastic and thermal properties of poly(vinylidene Fluoride)/POSS nanocomposites. Mater. Sci. Eng. C 2012, 32, 146-151. [CrossRef]

51. Liu, L.; Tian, M.; Zhang, W.; Zhang, L.; Mark, J.E. Crystallization and morphology study of polyhedral oligomeric silsesquioxane (POSS)/polysiloxane elastomer composites prepared by melt blending. Polymer 2007, 48, 3201-3212. [CrossRef]

52. Schadler, L.; Giannaris, S.a.; Ajayan, P. Load transfer in carbon nanotube epoxy composites. Appl. Phys. Lett. 1998, 73, 3842-3844. [CrossRef]

53. Gojny, F.; Wichmann, M.; Köpke, U.; Fiedler, B.; Schulte, K. Carbon nanotube-reinforced epoxy-composites: Enhanced stiffness and fracture toughness at low nanotube content. Compos. Sci. Technol. 2004, 64, 2363-2371. [CrossRef]

54. Kopesky, E.T.; McKinley, G.H.; Cohen, R.E. Toughened poly(methyl methacrylate) nanocomposites by incorporating polyhedral oligomeric silsesquioxanes. Polymer 2006, 47, 299-309. [CrossRef]

55. Špírková, M.; Duchek, P.; Strachota, A.; Poręba, R.; Kotek, J.; Baldrian, J.; Šlouf, M. The role of organic modification of layered nanosilicates on mechanical and surface properties of organic-inorganic coatings. J. Coat. Technol. Res. 2011, 8, 311-328. [CrossRef]

(C) 2018 by the authors. Licensee MDPI, Basel, Switzerland. This article is an open access article distributed under the terms and conditions of the Creative Commons Attribution (CC BY) license (http:/ / creativecommons.org/licenses/by/4.0/). 
Article

\title{
Improving the Mechanical and Electrical Properties of Ceramizable Silicone Rubber/Halloysite Composites and Their Ceramic Residues by Incorporation of Different Borates
}

\author{
Jianhua Guo ${ }^{1,2, *}$, Xuming Chen ${ }^{1}$ and Yong Zhang ${ }^{1}$ \\ 1 School of Materials Science and Engineering, South China University of Technology, Guangzhou 510640, \\ China; chenxm233@126.com (X.C.); tropo@foxmail.com (Y.Z.) \\ 2 Institute of Modern Industrial Technology of SCUT in Zhongshan, Zhongshan 528400, China \\ * Correspondence: psjhguo@scut.edu.cn
}

Received: 9 March 2018; Accepted: 27 March 2018; Published: 1 April 2018

\begin{abstract}
Ceramizable silicone rubber (MVQ)/halloysite (HNT) composites were fabricated by incorporation of three different borates, including sodium tetraborate decahydrate, ammonium pentaborate, and zinc borate into MVQ matrix, respectively. The composites without any borates were also prepared as control. The effect of the borates on the mechanical and electrical properties of MVQ/HNT composites was investigated. The ceramic residues were obtained from the decomposition of the composites after sintering at $1000^{\circ} \mathrm{C}$. The effect of the borates on the linear shrinkage, weight loss, and flexural and impact strength of the residues was also studied. The fracture surfaces of the composites and their corresponding residues were observed by SEM. The proposed ceramizable mechanism of the composites by incorporation of different borates was revealed by XRD analysis.
\end{abstract}

Keywords: ceramizable silicone rubber; borate; halloysite; composite; ceramizable mechanism

\section{Introduction}

Ceramizable silicone rubber (MVQ)-based composites have good processability like common elastomers at room temperature and transform into hard ceramic residues in fire environments or at elevated temperatures, which are desired materials to fulfill the increasing requirements in special applications such as fire resistant wires and cables [1]. Commonly, ceramizable silicone rubber-based composites are composed by polysiloxane elastomer, mineral fillers (montmorillonite, kaolin, talc, mica, etc.) [2-7], fluxing agents (glass frit, ammonium polyphosphate, etc.) [8-10], and other additives. Herein, fluxing agents are important components for ceramizable MVQ-based composites, which will be melted at elevated temperatures to merge some residues derived from the pyrolysis products of the composites. Furthermore, fluxing agent can promote low temperature ceramization and improve the mechanical strength of the ceramic residues [11-13]. At present, many studies have been conducted on the properties of ceramizable MVQ-based composites with the addition of glass frits with low softening point temperature. For example, Mansouri et al. [14] found that silicone rubber by incorporation of $2.5 \mathrm{wt} \%$ glass frits with a softening point temperature of $525^{\circ} \mathrm{C}$ can increase the flexural strength of the residues from 0.42 to $2.54 \mathrm{MPa}$ after firing at $800^{\circ} \mathrm{C}$. Guo et al. [15] showed that the flexural strength of ceramic residues after sintering at $1000{ }^{\circ} \mathrm{C}$ increased from 2 to $13.3 \mathrm{MPa}$ when the loading of glass frits with a softening point temperature of $480^{\circ} \mathrm{C}$ increased from 0 to $30 \mathrm{phr}$. However, the addition of glass frits will lead to a decrease of thermal stability of silicone rubber due to the catalyzed degradation effect of metal oxides decomposed from the glass frits [16]. Thus, some other fluxing agents except for glass frits are urgently needed to prepare ceramizable MVQ-based composites. 
It was reported that zinc borate (ZB) can act as flame retardant, smoke suppressant, and antitracking agent in both halogen-containing and halogen-free polymers [17]. The presence of ZB can also result in a significant char residue formation. Moreover, ZB plays a role of glass phase, helping to form a bridge between polymer matrix and mineral fillers, which is used extensively in polyvinyl chloride, polyamide, polyolefin, epoxy, phenolic, and poly(vinyl acetate) (PVAc) [18]. As we know that boron oxide $\left(\mathrm{B}_{2} \mathrm{O}_{3}\right)$ will be generated by the decomposition of zinc borate after heating, which provides afterglow suppression in polymers. Moreover, $\mathrm{B}_{2} \mathrm{O}_{3}$ will form glassy protective surface at $325^{\circ} \mathrm{C}$, which can act as a fluxing agent [19]. For example, Anyszka et al. [20] showed that the compressive strength of ceramic residues after firing at $1000{ }^{\circ} \mathrm{C}$ increased by more than four times when the loading of $\mathrm{B}_{2} \mathrm{O}_{3}$ increased from 20 to $40 \mathrm{phr}$. Halloysite nanotubes (HNTs) is a natural silicate mineral, consisting of 1:1 aluminosilicate layers with a tubular structure. Due to their special morphology, chemical structure, and surface properties, HNTs are widely used in fine ceramics [21], supported nanoparticles [22], and polymer composites [23]. However, HNTs acting as ceramic fillers and different borates acting as fluxing agents applied in MVQ have not been investigated in detail.

In this study, MVQ/HNT composites by incorporation of sodium tetraborate decahydrate, ammonium pentaborate, and zinc borate respectively, were fabricated and the composites without any fluxing agents were also prepared as control. The effect of the borates on the mechanical and electrical properties of ceramizable MVQ/HNT composites and the flexural and impact strength of the ceramic residues were investigated. Furthermore, the morphology of the ceramic residues was characterized by digital camera and SEM, and the proposed ceramic mechanism of MVQ-based composites was revealed by XRD.

\section{Experimental}

\subsection{Materials}

Methyl vinyl silicone rubber (MVQ, model 110-2, with a molecular weight of 600,000) was purchased from Caiyan Co., Ltd., Guangdong, China. Fumed silica (CABOSIL M-5) was purchased from Cabot Co., Ltd., Boston, MA, USA. Halloysites (HNTs) have a length in the range of 0.5-3.0 microns, an exterior diameter in the range of 50-70 nanometers and an internal diameter (lumen) in the range of $15-30$ nanometers. HNTs were produced by Linshounanyi Mineral Factory, Hebei, China. Sodium tetraborate decahydrate $\left(\mathrm{Na}_{2} \mathrm{~B}_{4} \mathrm{O}_{7} \cdot 10 \mathrm{H}_{2} \mathrm{O}\right)$ was purchased from Fuchen Chemical Reagent Factory, Tianjin, China. Ammonium pentaborate $\left(\mathrm{NH}_{4} \mathrm{~B}_{5} \mathrm{O}_{8} \cdot 4 \mathrm{H}_{2} \mathrm{O}\right)$ was purchased from Maclin Biochemical Sci. \& Tech. Co., Ltd., Shanghai, China. Zinc borate $\left(2 \mathrm{ZnO} \cdot 3 \mathrm{~B}_{2} \mathrm{O}_{3} \cdot 3.5 \mathrm{H}_{2} \mathrm{O}\right)$ was purchased from Guanghua Sci. \& Tech. Co., Ltd., Guangdong, China. Calcium carbonate was supplied by Enping Yanhua Industrial Co., Ltd., Guangdong, China. 2,5-dimethyl-2,5-bis (tert butyl peroxy) hexane (DBPMH, model C-15) was supplied by Caiyuan Silicone Materials Co., Ltd., Guangdong, China. The formulations of MVQ/HNT composites are summarized in Table 1. The formulation symbols are labeled as $\mathrm{NaB}, \mathrm{NHB}$ and $\mathrm{ZB}$ for the composites by incorporation of sodium tetraborate decahydrate, ammonium pentaborate, and zinc borate, respectively. 
Table 1. The formulations of ceramizable MVQ/HNT composites.

\begin{tabular}{ccccc}
\hline \multirow{2}{*}{ Ingredients (phr) } & \multicolumn{4}{c}{ Symbols } \\
\cline { 2 - 5 } & Control & NaB & NHB & ZB \\
\hline Silicone rubber & 100 & 100 & 100 & 100 \\
Fumed silica & 40 & 40 & 40 & 40 \\
Halloysite & 30 & 30 & 30 & 30 \\
Calcium carbonate & 5 & 5 & 5 & 5 \\
Sodium tetraborate decahydrate & - & 7.5 & - & - \\
Ammonium pentaborate & - & - & 7.5 & - \\
Zinc borate & - & - & - & 7.5 \\
DBPMH & 2 & 2 & 2 & 2 \\
\hline
\end{tabular}

\subsection{Preparation of Silicone Rubber Based Ceramizable Composites}

All of the compounds were prepared on a two-roll mill (model XK-160, Zhanjiang Machinery Factory, Guangdong, China) with a gear ratio of 1:1.4 at room temperature. The polysiloxane elastomer was first softened, and then the fumed silica, calcium carbonate, halloysites, and borates were added until a visually good dispersion was achieved. The curing agent DBPMH was then added and processed until a homogenous batch was obtained. The total mixing time is about $20 \mathrm{~min}$. The silicone rubber compounds were cured with flat sheets by compression molding in a hydraulic press (Model KSHR100T, Kesheng Industrial Co., Ltd., Guangdong, China) at $170{ }^{\circ} \mathrm{C}$ for 15 min under $15 \mathrm{MPa}$ pressure. The post curing of samples was carried out in an oven at $180^{\circ} \mathrm{C}$ for $2 \mathrm{~h}$. The ceramizing of flat sheet samples ( $50 \mathrm{~mm}$ long, $4 \mathrm{~mm}$ thick, and $6 \mathrm{~mm}$ wide) was performed using a muffle furnace (Shenjia KL-12B, Luoyang, China). The samples were heated from room temperature to $1000{ }^{\circ} \mathrm{C}$ at a heating rate of $10^{\circ} \mathrm{C} \cdot \mathrm{min}^{-1}$, held for $30 \mathrm{~min}$, and then cooled with the muffle furnace.

\subsection{Characterization of the Composites and Ceramic Residues}

The flexural strength of the ceramic residues was determined by a flexural method using a universal testing machine (Zwick/Roell Z010, Ulm, Germany) with a cross-head speed of $0.5 \mathrm{~mm} \cdot \mathrm{min}^{-1}$ according to ASTM D790-10. The impact strength was evaluated on an electronic Charpy impact tester (Suns, Shenzhen, China) according to ASTM D256-10e1. The weight loss of the ceramic residues was calculated according to Equation (1), where $W$ is the weight loss, and $m_{1}$ and $m_{2}$ are the mass of the samples before and after sintering, respectively.

$$
W=\left(m_{1}-m_{2}\right) / m_{1} \times 100 \%
$$

The shrinkage of the ceramic residue was measured by heating flat sheet samples to $1000{ }^{\circ} \mathrm{C}$ for $30 \mathrm{~min}$ in a muffle furnace. The linear shrinkage of the ceramic residue was calculated according to Equation (2), where $L$ is the linear shrinkage, and $l_{1}$ and $l_{2}$ are the length of the samples before and after sintering, respectively.

$$
L=\left(l_{1}-l_{2}\right) / l_{2} \times 100 \%
$$

The surfaces of the ceramizable MVQ/HNT composites and corresponding ceramic residues were observed by a digital camera (Canon 600D, Tokyo, Japan). The tensile fracture surface of the composites and the impact fracture surface of the ceramic residues were sprayed with gold and then observed by scanning electron microscopy (SEM, Zeiss EVO18, Oberkochen, Germany). Thermal gravity analysis (TGA) was performed by using TG 309F1 instrument (Netzsch, Selb, Germany). Samples (weight $8 \sim 10 \mathrm{mg}$ ) were heated at a heating rate of $10{ }^{\circ} \mathrm{C} \cdot \mathrm{min}^{-1}$ from 30 to $900{ }^{\circ} \mathrm{C}$ under nitrogen atmosphere. X-ray diffraction spectra of the ceramic residues were obtained using an X-ray diffractometer (XRD, PANlytical X'pert, Almelo, The Netherlands). Each scan was conducted from a $2 \theta$ angle of $5^{\circ} \sim 75^{\circ}$ at a scan rate of $1^{\circ} \cdot \mathrm{min}^{-1}$. 


\section{Results and Discussions}

\subsection{Characterization of the Borates}

Halloysites (HNTs) act as the ceramic fillers in the MVQ composites, with the similar function as kaolin, mica, talc, etc. Some borates including sodium tetraborate decahydrate (NaB), ammonium pentaborate (NHB), and zinc borate (ZB), serve as the fluxing agents. The SEM photographs of three borates were showed in Figure 1. It can be observed in Figure 1a that the NaB particles are clustered at certain area and form aggregates. The average size of the NaB particles is about $200 \mu \mathrm{m}$. It is found that the NHB particles are spherical in shape with an average diameter of $50 \mu \mathrm{m}$ (Figure 1b). However, the ZB particles have sharper edges than the $\mathrm{NaB}$ and NHB particles as depicted in Figure 1c. The ZB particles are irregular with a much smaller size of about $1 \sim 3 \mu \mathrm{m}$.
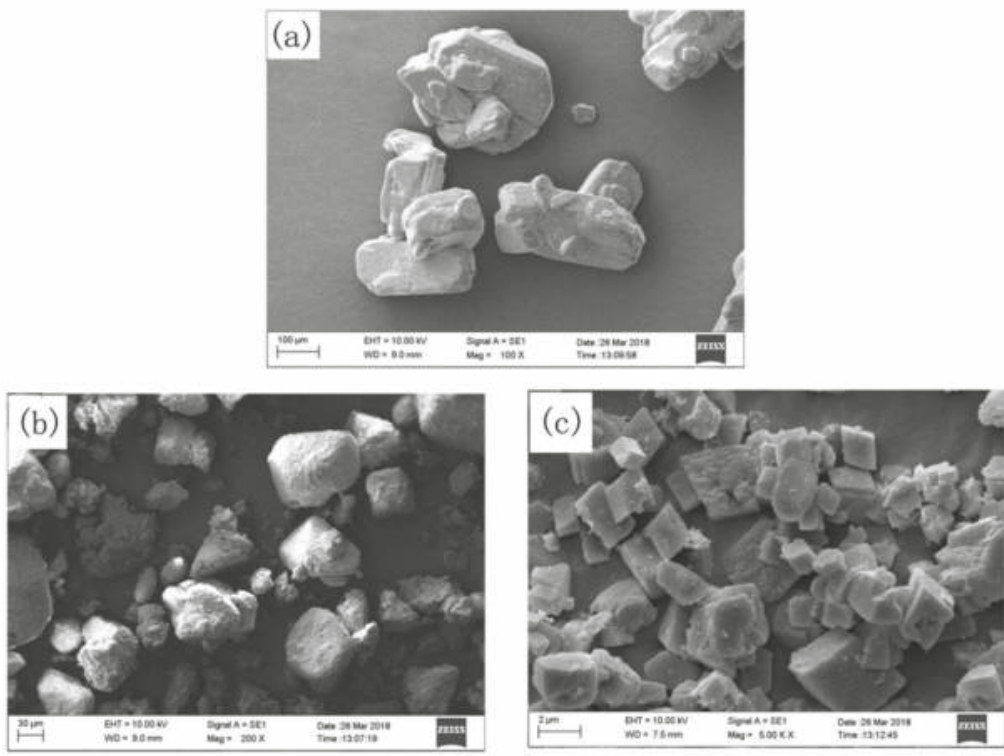

Figure 1. SEM photographs of (a) sodium tetraborate decahydrate, (b) ammonium pentaborate, and (c) zinc borate.

Thermal stability of sodium tetraborate decahydrate, ammonium pentaborate, and zinc borate was investigated in Figure 2, and the corresponding thermal parameters are listed in Table 2. It is shown that the initial decomposition temperature $\left(\mathrm{T}_{5 \%}\right)$ of sodium tetraborate decahydrate, ammonium pentaborate, and zinc borate are $66.9,144.0$, and $371.5^{\circ} \mathrm{C}$, respectively. The maximum degradation temperature $\left(\mathrm{T}_{\mathrm{d} \%}\right)$ of zinc borate is much higher than that of sodium tetraborate decahydrate and ammonium pentaborate. The mass of the residues obtained from the decomposition of sodium tetraborate decahydrate, ammonium pentaborate and zinc borate at $900{ }^{\circ} \mathrm{C}$ is $54.2,64.6$, and $84.3 \%$, respectively. Zinc borate, sodium tetraborate decahydrate, and ammonium pentaborate have one, two, and three decomposition process, respectively. The mass loss of first decomposition of sodium tetraborate decahydrate, ammonium pentaborate and zinc borate is 35.2, 20.2 and 15.5\% respectively, which is attributed to the removal of crystal water within their chemical structure. Thus, zinc borate shows better thermal stability than sodium tetraborate decahydrate and ammonium pentaborate. 


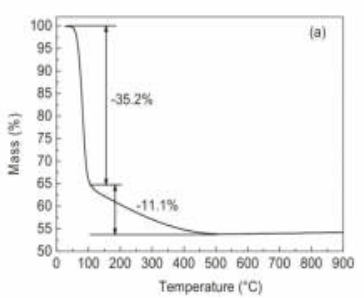

(a)

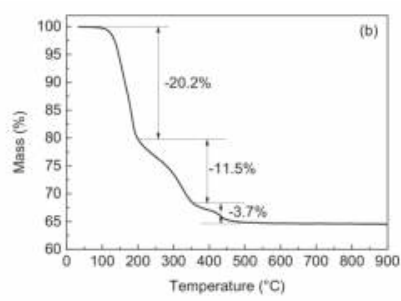

(b)

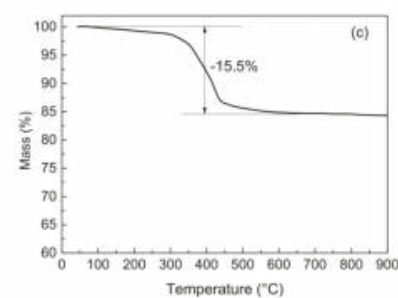

(c)

Figure 2. TGA curves of (a) sodium tetraborate decahydrate, (b) ammonium pentaborate, and (c) zinc borate.

Table 2. Thermogravimetric analysis of $\mathrm{NaB}, \mathrm{NHB}$, and ZB fillers.

\begin{tabular}{cccc}
\hline Samples & Initial Degradation Temperature, $\mathbf{T}_{5 \%}\left({ }^{\circ} \mathrm{C}\right)$ & Maximum Degradation Temperature, $\mathbf{T}_{\mathbf{d} \%}\left({ }^{\circ} \mathrm{C}\right)$ & Mass of Residue at $900{ }^{\circ} \mathrm{C}(\%)$ \\
\hline $\mathrm{NaB}$ & 66.9 & 84.0 & 54.2 \\
$\mathrm{NHB}$ & 144.0 & 184.0 & 64.6 \\
$\mathrm{ZB}$ & 371.5 & 417.0 & 84.3 \\
\hline
\end{tabular}

Note: NaB: sodium tetraborate decahydrate; NHB: ammonium pentaborate; ZB: zinc borate.

\subsection{Effect of Borates on the Mechanical Properties of Ceramizable MVQ/HNT Composites}

The tensile stress-strain curves of ceramizable MVQ-based composites were shown in Figure 3. For the control, the tensile strength and elongation at break are $4.9 \mathrm{MPa}$ and $469 \%$, respectively. The tensile strength and elongation at break of the composites with the addition of sodium tetraborate decahydrate (abbr. as $\mathrm{NaB}$ composite) and ammonium pentaborate (abbr. as NHB composite) decrease, compared with the control. However, the tensile strength of the MVQ/HNT composite by incorporation of zinc borate (abbr. as ZB composite) is $5.5 \mathrm{MPa}$, which is $72 \%$ and $45 \%$ higher than that of the $\mathrm{NaB}$ and $\mathrm{NHB}$ composites respectively. According to the TGA results in Figure 2, the thermal decomposition of $\mathrm{NaB}$ and $\mathrm{NHB}$ fillers leads to the releasing of water steam during the curing process of the MVQ composites at $170^{\circ} \mathrm{C}$ to form some voids or flaws in the MVQ matrix. The voids will result in a decrease in the mechanical properties of the MVQ composites. However, because of the higher initial decomposition temperature of $Z \mathrm{~B}$, it is easier to form dense structure in the MVQ composites than another two borates. Furthermore, another reason is that $\mathrm{ZB}$ in the composites might cause an increase in the physical crosslinking centers due to the higher electronegativity of divalent zinc ions than other elements in the blend [24], leading to an increase in the mechanical properties.

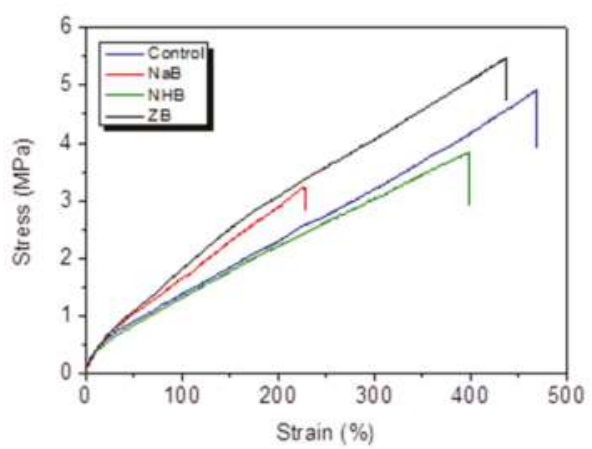

Figure 3. Tensile stress-strain curves of ceramizable MVQ/HNT composites with the addition of different borates. 


\subsection{Effect of Borates on the Electrical Properties of Ceramizable MVQ/HNT Composites}

The volume and surface resistivity of ceramizable MVQ/HNT composites with different borates were shown in Figure 4. For the control, the volume and surface resistivity are $1.36 \times 10^{13} \Omega \cdot \mathrm{m}$ and $2.08 \times 10^{13} \Omega$, respectively. By incorporation of the borates, the electrical resistivity of the composites increase to some extent. Particularly, the ZB composite shows the maximum volume and surface resistivity with the values of $1.65 \times 10^{14} \Omega \cdot \mathrm{m}$ and $7.88 \times 10^{14} \Omega$ respectively, indicating that zinc borate will significantly improve the electrical insulation properties of MVQ. Similarly, zinc borate can improve the electrical insulation properties of ethylene-vinyl acetate copolymer (EVA)/metal hydroxide systems [17] and linear low-density polyethylene/rubbers/magnesium oxide composite [24]. These results may be attributed to collision of electrons by ZB particles inside the matrix which would obstruct the electron avalanche in the composites, and more obstruction would be generated finally resulting in higher volume and surface resistivity [24].

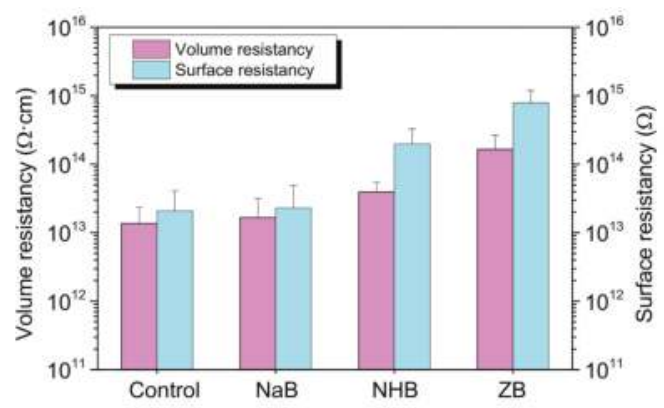

Figure 4. Electrical insulation properties of ceramizable MVQ/HNT composites with the addition of different borates.

\subsection{Effect of Borates on Linear Shrinkage and Weight Loss of Ceramic Residues}

Linear shrinkage and weight loss of the ceramic residues obtained from the decomposition of the MVQ-based composites by incorporation of different borates were shown in Figure 5. The linear shrinkage of ceramic residues with the addition of different borates is bigger than that of the control because $\mathrm{B}_{2} \mathrm{O}_{3}$ can act as a fluxing agent, which is derived from the thermal decomposition of the borates. Then $\mathrm{SiO}_{2}$ generated from the degradation of MVQ was assembled to form a compact integration due to the adhesion of melted $\mathrm{B}_{2} \mathrm{O}_{3}$ after sintering at $1000{ }^{\circ} \mathrm{C}$, resulting in a decrease in the length and volume of the residues. However, the weight loss of the MVQ composites with the addition of different borates is similar, which is because the weight percents of the inorganic fillers such as the borates and halloysites are almost the same.

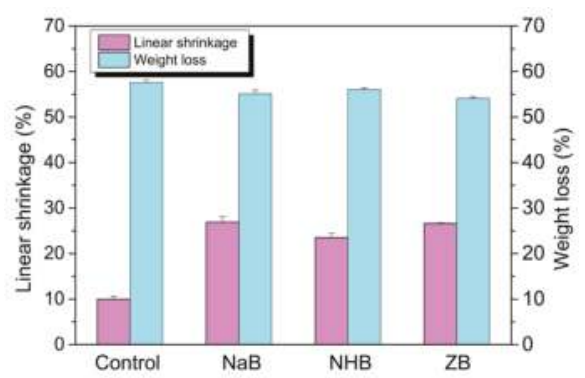

Figure 5. Linear shrinkage and weight loss of the ceramic residues by incorporation of different borates. 


\subsection{Surface Morphology of Ceramizable MVQ/HNT Composites and Corresponding Ceramic Residues}

The surface morphology of the ceramizable MVQ/HNT composites and corresponding ceramic residues with the addition of different borates was shown in Figure 6. It is exhibited that the control sample and ZB composite is relatively smooth, however, the surfaces of the $\mathrm{NaB}$ and $\mathrm{NHB}$ composites are rough and dispersed with some white dots. This is because the poor compatibility of sodium tetraborate decahydrate and ammonium pentaborate with MVQ matrix. The corresponding ceramic residues by incorporation of different borates show great linear shrinkage, compared with the control. Moreover, the length of the residue derived from the NHB composite is a little longer than that of another two residues derived from the $\mathrm{NaB}$ and $\mathrm{ZB}$ composites, respectively. This is because the releasing of ammonia and water stream during the decomposition of ammonium pentaborate leads to an increase in the porosity of ceramic residues and then a decrease in the linear shrinkage. The color of the composites turns from gray (before firing) to pink (after firing), which might be due to some metal oxides such as $\mathrm{Fe}_{2} \mathrm{O}_{3}$ derived from the impurities in HNTs.

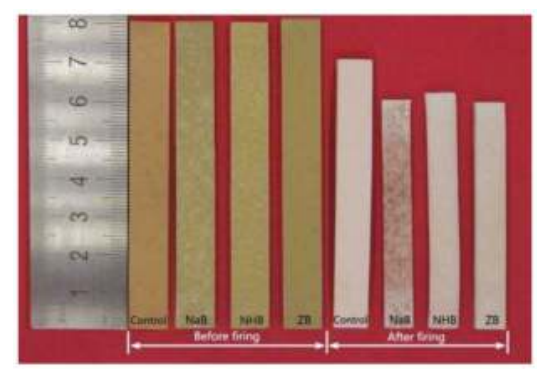

Figure 6. Surface morphology of ceramizable MVQ/HNT composites and corresponding ceramic residues obtained from the pyrolysis products of the composites with the addition of different borates.

\subsection{Effect of the Borates on the Flexural and Impact Strength of Ceramic Residues}

Effect of the borates on the flexural and impact strength of ceramic residues was shown in Figure 7. For the control sample, the flexural and impact strength is $2.9 \mathrm{MPa}$ and $2.1 \mathrm{~J} \cdot \mathrm{m}^{-1}$, respectively. The residues derived from the $\mathrm{ZB}$ composites exhibit the maximum flexural and impact strength with the values of $28.7 \mathrm{MPa}$ and $4.5 \mathrm{~J} \cdot \mathrm{m}^{-1}$, which are $890 \%$ and $114 \%$ higher than those of the control, respectively. This is because the high thermal stability of zinc borate helps to form dense ceramic structure with improved ceramic strength. However, the residues derived from the NHB composites show the minimum flexural and impact strength with the values of $13 \mathrm{MPa}$ and $2.7 \mathrm{~J} \cdot \mathrm{m}^{-1}$ respectively, which is due to the releasing of ammonia and crystal water with the decomposition of ammonium pentaborate, destroying the dense structure of the residues.

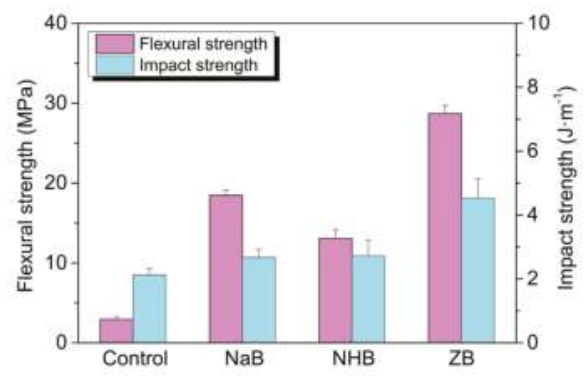

Figure 7. Effect of three borates on the flexural and impact strength of ceramic residues. 


\subsection{SEM Analysis}

The tensile fractures of the control (Figure 8a), ceramizable NaB (Figure 8b), NHB (Figure 8c), and ZB (Figure 8d) composites and the impact fractures of the ceramic residues derived from the decomposition of the composites were shown in Figure 8. Before sintering, the tensile fractures of all the composites are smooth and dense. However, it is found that there are more holes in the impact fractures of the residues containing the borates than that of the control residue (Figure 8e). However, there are fewer cavities in the impact fracture of the $\mathrm{ZB}$ residue (Figure $8 \mathrm{~h}$ ) than those of $\mathrm{NaB}$ (Figure 8f) and $\mathrm{NHB}$ (Figure $8 \mathrm{~g}$ ) residues, indicating that more $\mathrm{B}_{2} \mathrm{O}_{3}$ (liquid phase) generated by the decomposition of zinc borate makes the ceramic residues denser. The releasing of more crystalliferous water or ammonia accompanied by the decomposition of sodium tetraborate decahydrate and ammonium pentaborate might result in many holes in the fracture surface.
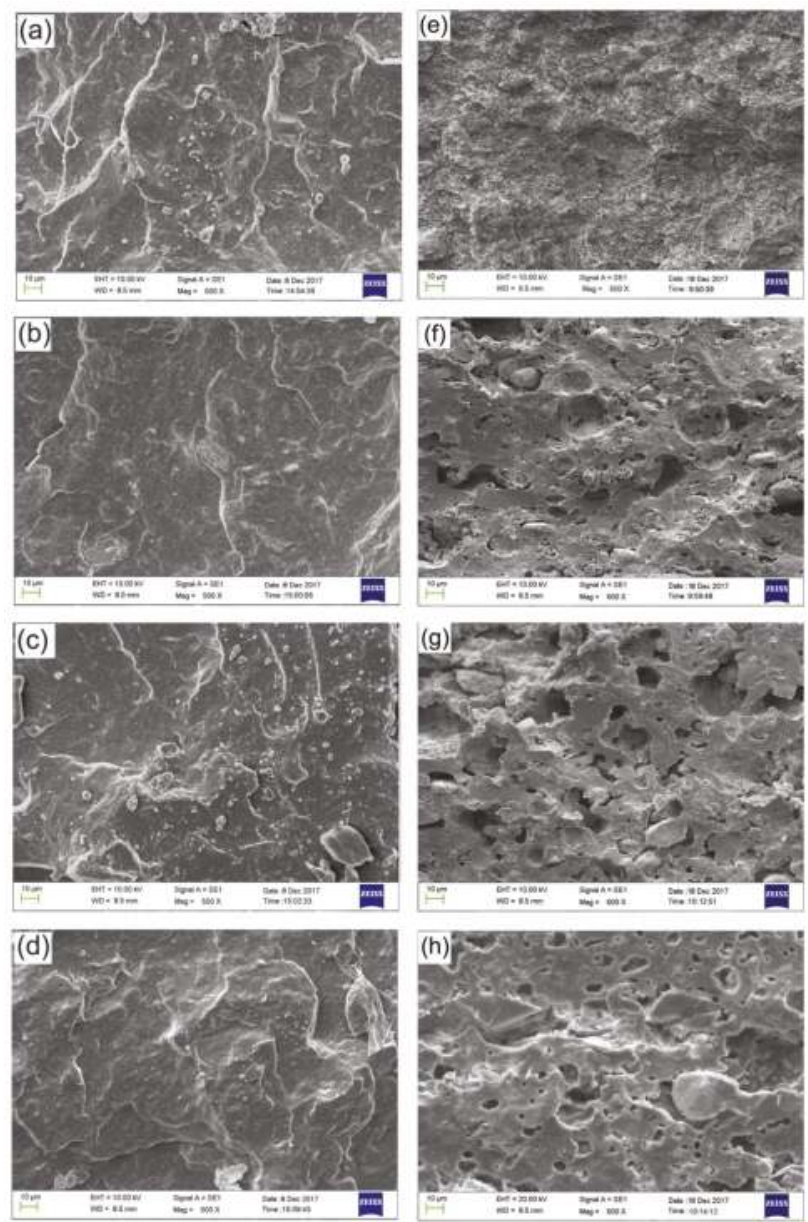

Figure 8. Morphology of tensile fracture surfaces of (a) the control and ceramizable, (b) NaB, (c) NHB, and (d) ZB composites and impact fracture surfaces of (e) the ceramic residue obtained from the control composite and the ceramic ones derived from the decomposition of the (f) NaB, (g) NHB, and (h) ZB composites, respectively. 


\subsection{XRD Analysis}

XRD spectra of the ceramic residues obtained from the decomposition of the $\mathrm{NaB}, \mathrm{NHB}$, and $\mathrm{ZB}$ composites after sintering at $1000{ }^{\circ} \mathrm{C}$ was shown in Figure 9. The residues without any borates were chosen as control. For the control residue, there is no diffraction peaks in the XRD spectrum, indicating no crystalline phases existed in the residues. For the residues with the addition of the borates, the main crystalline phases [25,26] include quartz $\left(\mathrm{SiO}_{2}\right)(\mathrm{JCPDS}$ no. 46-1045) and cristobalite (JCPDS no. 76-1390), anorthite $\left(\mathrm{CaAl}_{2} \mathrm{Si}_{2} \mathrm{O}_{8}\right)$ (JCPDS no. 41-1486) and mullite $\left(\mathrm{Al}_{6} \mathrm{Si}_{2} \mathrm{O}_{13}\right)$ (JCPDS no. 15-0776). It is known that amorphous silica $\left(\mathrm{SiO}_{2}\right)$ was obtained from pyrolysis of MVQ, and alumina $\left(\mathrm{Al}_{2} \mathrm{O}_{3}\right)$ was obtained from the decomposition of halloysites and calcium oxide $(\mathrm{CaO})$ was obtained from the degradation of calcium carbonate $\left(\mathrm{CaCO}_{3}\right)$ in the sintering process. Because the eutectic reactions were triggered within $\mathrm{SiO}_{2}, \mathrm{Al}_{2} \mathrm{O}_{3}$, and $\mathrm{CaO}$, some new crystals such as cristobalite, quartz, anorthite, and mullite emerged after cooling of the residues. However, for the residue derived from the $\mathrm{ZB}$ composite, the diffraction peaks $\left(2 \theta=31.227^{\circ}, 36.772^{\circ}\right)$ appear in the XRD spectra, indicating the generation of gahnite $\left(\mathrm{ZnAl}_{2} \mathrm{O}_{4}\right)$ (JCPDS no. 05-0669), which is due to the eutectic reaction between $\mathrm{ZnO}$ and $\mathrm{Al}_{2} \mathrm{O}_{3}$.

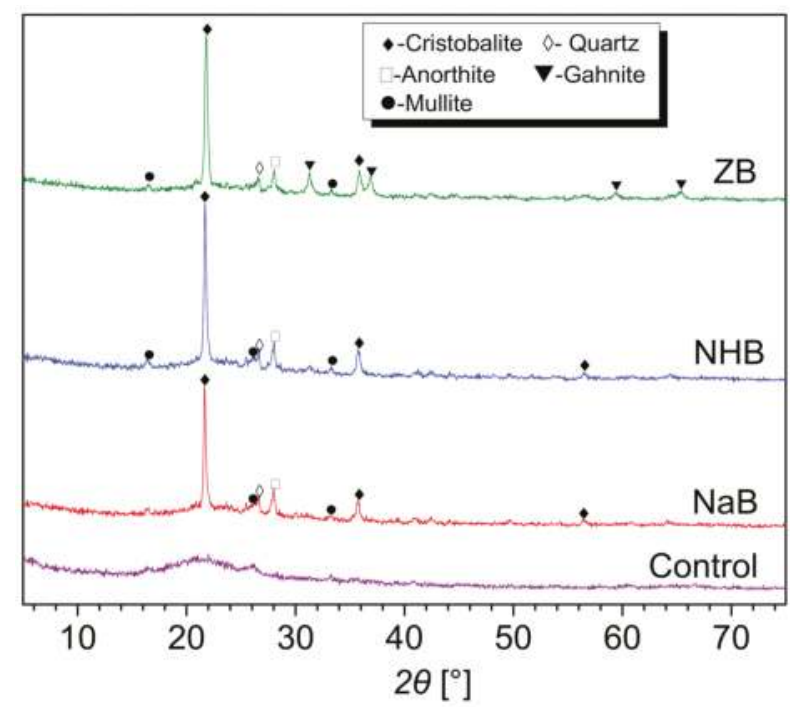

Figure 9. XRD spectra of the ceramic residues obtained from the MVQ/HNT composites with the addition of different borates.

\subsection{Proposed Ceramizable Mechanism}

The decomposition of three berates and proposed eutectic reactions between different metal oxides are shown in Equations (3)-(14). According to the TGA results in Figure 2, sodium tetraborate decahydrate $\left(\mathrm{Na}_{2} \mathrm{~B}_{4} \mathrm{O}_{7} \cdot 10 \mathrm{H}_{2} \mathrm{O}\right)$ is white crystal, which will be decomposed to $\mathrm{B}_{2} \mathrm{O}_{3}$ at elevated temperature. Ammonium pentaborate $\left(\mathrm{NH}_{4} \mathrm{~B}_{5} \mathrm{O}_{8} \cdot 4 \mathrm{H}_{2} \mathrm{O}\right)$, which is decomposed to $\mathrm{B}_{2} \mathrm{O}_{3}$ at the temperature up to $470{ }^{\circ} \mathrm{C}$. Zinc borate $\left(2 \mathrm{ZnO} \cdot 3 \mathrm{~B}_{2} \mathrm{O}_{3} \cdot 3.5 \mathrm{H}_{2} \mathrm{O}\right)$ can act as an inorganic boron-containing flame retardant, which is decomposed to $\mathrm{B}_{2} \mathrm{O}_{3}$ from 370 to $450{ }^{\circ} \mathrm{C}$ [20]. The thermal decomposition of halloysites involves dehydroxylation at $500 \sim 900^{\circ} \mathrm{C}$, the silica and alumina are originally formed in the tetrahedral and octahedral sheets, respectively. Nano sized $(5-40 \mathrm{~nm}) \gamma-\mathrm{Al}_{2} \mathrm{O}_{3}$ is generated at $900 \sim 1000{ }^{\circ} \mathrm{C}$ [27]. There are eutectic reactions between some metal oxides such as $\mathrm{Al}_{2} \mathrm{O}_{3}, \mathrm{SiO}_{2}$, $\mathrm{CaO}$, and $\mathrm{ZnO}$ to generate anorthite $\left(\mathrm{CaAl}_{2} \mathrm{Si}_{2} \mathrm{O}_{8}\right)$, mullite $\left(\mathrm{Al}_{6} \mathrm{Si}_{2} \mathrm{O}_{13}\right)$, gahnite $\left(\mathrm{ZnAl}_{2} \mathrm{O}_{4}\right)$, etc. All the crystals will strengthen the ceramic residues and increase their flexural and impact strength [28]. 
Decomposition of sodium tetraborate decahydrate:

$$
\begin{gathered}
70 \sim 100{ }^{\circ} \mathrm{C} \mathrm{Na}_{2} \mathrm{~B}_{4} \mathrm{O}_{7} \cdot 10 \mathrm{H}_{2} \mathrm{O} \rightarrow \mathrm{Na}_{2} \mathrm{~B}_{4} \mathrm{O}_{7}+10 \mathrm{H}_{2} \mathrm{O} \uparrow \\
100 \sim 500{ }^{\circ} \mathrm{C} \mathrm{Na}_{2} \mathrm{~B}_{4} \mathrm{O}_{7} \rightarrow 2 \mathrm{NaBO}_{2}+\mathrm{B}_{2} \mathrm{O}_{3}
\end{gathered}
$$

Decomposition of ammonium pentaborate:

$$
\begin{aligned}
100 \sim 200{ }^{\circ} \mathrm{C} \mathrm{NH}_{4} \mathrm{~B}_{5} \mathrm{O}_{8} \cdot 4 \mathrm{H}_{2} \mathrm{O} & \rightarrow \mathrm{NH}_{4} \mathrm{~B}_{5} \mathrm{O}_{8} \cdot \mathrm{H}_{2} \mathrm{O}+3 \mathrm{H}_{2} \mathrm{O} \uparrow \\
200 \sim 350{ }^{\circ} \mathrm{C} \mathrm{NH}_{4} \mathrm{~B}_{5} \mathrm{O}_{8} \cdot \mathrm{H}_{2} \mathrm{O} & \rightarrow \mathrm{HB}_{5} \mathrm{O}_{8}+\mathrm{H}_{2} \mathrm{O} \uparrow+\mathrm{NH}_{3} \uparrow \\
350 \sim 470{ }^{\circ} \mathrm{C} 2 \mathrm{HB}_{5} \mathrm{O}_{8} & \rightarrow 5 \mathrm{~B}_{2} \mathrm{O}_{3}+\mathrm{H}_{2} \mathrm{O} \uparrow
\end{aligned}
$$

Decomposition of zinc borate:

$$
370 \sim 500{ }^{\circ} \mathrm{C} 2 \mathrm{ZnO} \cdot 3 \mathrm{~B}_{2} \mathrm{O}_{3} \cdot 3.5 \mathrm{H}_{2} \mathrm{O} \rightarrow 2 \mathrm{ZnO}+3 \mathrm{~B}_{2} \mathrm{O}_{3}+3.5 \mathrm{H}_{2} \mathrm{O} \uparrow
$$

Transformation of silica:

$$
1000{ }^{\circ} \mathrm{C} \mathrm{SiO}_{2} \text { (amorphous) } \rightarrow \mathrm{SiO}_{2} \text { (crystal) }
$$

Decomposition of halloysites:

$$
\begin{gathered}
500 \sim 900^{\circ} \mathrm{C} \mathrm{Al}_{2}(\mathrm{OH})_{4} \mathrm{Si}_{2} \mathrm{O}_{5} \cdot 2 \mathrm{H}_{2} \mathrm{O} \text { (Halloysites) } \rightarrow \mathrm{Al}_{2} \mathrm{O}_{3} \cdot 2 \mathrm{SiO}_{2}+4 \mathrm{H}_{2} \mathrm{O} \uparrow \\
900 \sim 1000^{\circ} \mathrm{C} \mathrm{Al}_{2} \mathrm{O}_{3} \cdot 2 \mathrm{SiO}_{2} \rightarrow \mathrm{Al}_{2} \mathrm{O}_{3}+2 \mathrm{SiO}_{2}
\end{gathered}
$$

Proposed eutectic reactions:

$$
\begin{gathered}
2 \mathrm{SiO}_{2}+3 \mathrm{Al}_{2} \mathrm{O}_{3} \rightarrow \mathrm{Al}_{6} \mathrm{Si}_{2} \mathrm{O}_{13} \text { (mullite) } \\
\mathrm{CaO}+2 \mathrm{SiO}_{2}+\mathrm{Al}_{2} \mathrm{O}_{3} \rightarrow \mathrm{CaAl}_{2} \mathrm{Si}_{2} \mathrm{O}_{8} \text { (anorthite) } \\
\mathrm{ZnO}+\mathrm{Al}_{2} \mathrm{O}_{3} \rightarrow \mathrm{ZnAl}_{2} \mathrm{O}_{4} \text { (gahnite) }
\end{gathered}
$$

\section{Conclusions}

The mechanical and electrical properties of ceramizable silicone rubber (MVQ)-based composites with the addition of zinc borate are better than those of sodium tetraborate decahydrate and ammonium pentaborate. The tensile strength of MVQ/HNT composites with zinc borate is $69 \%$ and $42 \%$ bigger than that with sodium tetraborate decahydrate and ammonium pentaborate, respectively. The volume and surface resistivity of MVQ/HNT composites with zinc borate are higher than those with sodium tetraborate decahydrate and ammonium pentaborate, showing the values of $1.7 \times 10^{14} \Omega \cdot \mathrm{m}$ and $7.9 \times 10^{14} \Omega$, respectively. The flexural and impact strength of the ceramic residues derived from decomposition of the composites by incorporation of zinc borate are $28.7 \mathrm{MPa}$ and $4.5 \mathrm{~J} \cdot \mathrm{m}^{-1}$ respectively due to the dense structure in the residues. Furthermore, the eutectic reactions between zinc borate and halloysite will generate some crystals such as mullite and gahnite in the residues after sintering at $1000^{\circ} \mathrm{C}$, which further increases the flexural and impact strength of the residues.

Acknowledgments: This work was supported by the National Natural Science Foundation of China (grant no. 51103048), and Science and Technology Development Project of Zhongshan City (no. 2016F2FC0014), and Science and Technology Planning Project of Guangdong Province, China (no. 2016A010103001).

Author Contributions: Jianhua Guo conceived and designed the study and wrote the paper. Xuming Chen and Yong Zhang performed the experiments. All authors read and approved the manuscript.

Conflicts of Interest: The authors declare no conflict of interest. 


\section{References}

1. Hayashida, K.; Tsuge, S.; Ohtani, H. Flame retardant mechanism of polydimethylsiloxane material containing platinum compound studied by analytical pyrolysis techniques and alkaline hydrolysis gas chromatography. Polymer 2003, 44, 5611-5616. [CrossRef]

2. Hanu, L.G.; Simon, G.P.; Cheng, Y.B. Preferential orientation of muscovite in ceramizable silicone composites. Mater. Sci. Eng. A 2005, 398, 180-187. [CrossRef]

3. Mansouri, J.; Burford, R.P.; Cheng, Y.B.; Hanu, L. Formation of strong ceramified ash from silicone-based compositions. J. Mater. Sci. 2005, 40, 5741-5749. [CrossRef]

4. Gong, X.H.; Wu, T.Y.; Ma, J.; Zhao, D.; Shen, Y.C.; Wang, T.W. Improved self-supporting property of ceramifying silicone rubber composites by forming crystalline phase at high temperatures. J. Alloys Compd. 2017, 706, 322-329. [CrossRef]

5. Yu, L.; Zhou, S.; Zou, H.; Liang, M. Thermal stability and ablation properties study of aluminum silicate ceramic fiber and acicular wollastonite filled silicone rubber composite. J. Appl. Polym. Sci. 2014, 131, 1-15. [CrossRef]

6. Lou, F.P.; Yan, W.; Guo, W.H.; Wei, T.; Li, Q.Y. Preparation and properties of ceramifiable flame-retarded silicone rubber composites. J. Therm. Anal. Calorim. 2017, 130, 813-821. [CrossRef]

7. Anyszka, R.; Bieliński, D.M.; Pędzich, Z.; Szumera, M. Influence of surface-modified montmorillonites on properties of silicone rubber-based ceramizable composites. J. Therm. Anal. Calorim. 2015, 119, 111-121. [CrossRef]

8. Guo, J.H.; Zhang, Y.; Li, H.J.; Zhang, X. Effect of the sintering temperature on the microstructure, properties and formation mechanism of ceramic materials obtained from polysiloxane elastomer-based ceramizable composites. J. Alloys Compd. 2016, 678, 499-505. [CrossRef]

9. Lou, F.P.; Cheng, L.H.; Li, Q.Y.; Wei, T.; Guan, X.Y.; Guo, W.H. The combination of glass dust and glass fiber as fluxing agents for ceramizable silicone rubber composites. RSC Adv. 2017, 7, 38805-38811. [CrossRef]

10. Hu, S.; Chen, F.; Li, J.G.; Shen, Q.; Huang, Z.X.; Zhang, M.L. The ceramifying process and mechanical properties of silicone rubber/ammonium polyphosphate/aluminium hydroxide/mica composites. Polym. Degrad. Stabil. 2016, 126, 196-203. [CrossRef]

11. Hamdani, S.; Pommier, A.; Longuet, C.; Lopez-Cuesta, J.M.; Ganachaud, F. Calcium and aluminium-based fillers as flame-retardant additives in silicone matrices II. Analyses on composite residues from an industrial-based pyrolysis test. Polym. Degrad. Stabil. 2011, 96, 1562-1572. [CrossRef]

12. Imiela, M.; Anyszka, R.; Bieliński, D.M.; Pẹdzich, Z.; Napierała, M.Z.; Szumera, M. Effect of carbon fibers on thermal properties and mechanical strength of ceramizable composites based on silicone rubber. J. Therm. Anal. Calorim. 2016, 124, 197-203. [CrossRef]

13. Marosi, G.; Márton, A.; Anna, P.; Bertalan, G.; Marosföi, B.; Szép, A. Ceramic precursor in flame retardant systems. Polym. Degrad. Stabil. 2002, 77, 259-265. [CrossRef]

14. Mansouri, J.; Wood, C.; Roberts, K.; Cheng, Y.B.; Burford, R.P. Investigation of the ceramifying process of modified silicone-silicate compositions. J. Mater. Sci. 2007, 42, 6046-6055. [CrossRef]

15. Guo, J.H.; Gao, W.; Wang, Y.; Liang, D.; Li, H.J.; Zhang, X. Effect of glass frit with low softening temperature on the properties, microstructure and formation mechanism of polysiloxane elastomer-based ceramizable composites. Polym. Degrad. Stabil. 2017, 136, 71-79. [CrossRef]

16. Hanu, L.G.; Simon, G.P.; Cheng, Y.B. Thermal stability and flammability of silicone polymer composites. Polym. Degrad. Stabil. 2006, 91, 1373-1379. [CrossRef]

17. Shen, K.K.; Kochesfahani, S.; Jouffret, F. Zinc borates as multifunctional polymer additives. Polym. Adv. Technol. 2010, 19, 469-474. [CrossRef]

18. Al-Hassany, Z.; Genovese, A.; Shanks, R.A. Fire-retardant and fire-barrier poly(vinyl acetate) composites for sealant application. Express Polym. Lett. 2010, 4, 79-93. [CrossRef]

19. Anyszka, R.; Bielinski, D.M.; Jẹdrzejczyk, M. Thermal behavior of silicone rubber based ceramizable composites characterized by fourier transform infrared (FT-IR) spectroscopy and microcalorimetry. Appl. Spectrosc. 2013, 67, 1437-1440. [CrossRef] [PubMed]

20. Anyszka, R.; Bieliński, D.M.; Pędzich, Z.; Dul, J. Ceramizable silicone rubber-based composites. Adv. Sci. Technol. 2012, 66, 82-88.

21. Wilson, I.R. Kaolin and halloysite deposits of China. Clay Miner. 2004, 39, 1-15. [CrossRef] 
22. Lvov, Y.M.; Shchukin, D.G.; Möhwald, H.; Price, R.R. Halloysite clay nanotubes for controlled release of protective agents. ACS Nano 2008, 2, 814-820. [CrossRef] [PubMed]

23. Pasbakhsh, P.; Ismail, H.; Fauzi, M.N.A.; Bakar, A.A. EPDM/modified halloysite nanocomposites. Appl. Clay Sci. 2010, 48, 405-413. [CrossRef]

24. Alwaan, I.M.; Hassan, A.; Piah, M.A.M. Effect of zinc borate on mechanical and dielectric properties of metallocene linear low-density polyethylene/rubbers/magnesium oxide composite for wire and cable applications. Iran Polym. J. 2015, 24, 279-288. [CrossRef]

25. Yang, S.N.; Lv, G.P.; Liu, Y.; Wang, Q. Synergism of polysiloxane and zinc borate flame retardant polycarbonate. Polym. Degrad. Stabil. 2013, 98, 2795-2800. [CrossRef]

26. Wang, J.; Ji, C.; Yan, Y.; Zhao, D.; Shi, L. Mechanical and ceramizable properties of silicone rubber filled with different inorganic fillers. Polym. Degrad. Stabil. 2015, 121, 149-156. [CrossRef]

27. Yuan, P.; Tan, D.Y.; Annabi-Bergaya, F.; Yan, W.C.; Fan, M.D.; Liu, D.; He, H.P. Changes in structure, morphology, porosity, and surface activity of mesoporous halloysite nanotubes under heating. Clays Clay Miner. 2012, 60, 561-573. [CrossRef]

28. Kokubo, T.; Ito, S.; Sakka, S.; Yamamuro, T. Formation of a high-strength bioactive glass-ceramic in the system $\mathrm{MgO}-\mathrm{CaO}-\mathrm{SiO}_{2}-\mathrm{P}_{2} \mathrm{O}_{5}$. J. Mater. Sci. 1986, 21, 536-540. [CrossRef]

(C) 2018 by the authors. Licensee MDPI, Basel, Switzerland. This article is an open access article distributed under the terms and conditions of the Creative Commons Attribution (CC BY) license (http:/ / creativecommons.org/licenses/by/4.0/). 


\title{
Article \\ Effect of Polysiloxanes on Roughness and Durability of Basalt Fibres-Reinforced Cement Mortar
}

\author{
Danuta Barnat-Hunek ${ }^{1}$, Grzegorz Łagód ${ }^{2, *}$, Stanisław Fic ${ }^{1}$ and Monika Jarosz-Hadam ${ }^{3}$ \\ 1 Faculty of Civil Engineering and Architecture, Lublin University of Technology, Nadbystrzycka 40, \\ 20-618 Lublin, Poland; d.barnat-hunek@pollub.pl (D.B.-H.), s.fic@pollub.pl (S.F.) \\ 2 Faculty of Environmental Engineering, Lublin University of Technology, Nadbystrzycka 40B, \\ 20-618 Lublin, Poland \\ 3 Faculty of Economics and Engineering, Pope John Paul II State School of Higher Education in Biała \\ Podlaska, Sidorska 95/97, 21-500 Biała Podlaska, Poland; m.jarosz-hadam@dydaktyka.pswbp.pl \\ * Correspondence: g.lagod@pollub.pl; Tel.: +48-81-538-43-22 (ext. 4322)
}

Received: 15 March 2018; Accepted: 7 April 2018; Published: 9 April 2018

\begin{abstract}
The influence of roughness and the way it affects the adhesion properties and surface free energy (SFE) of polysiloxanes hydrophobised basalt fibres-reinforced cement mortars were determined in this article. The physical properties of mortars were investigated in the experimental part, which also explored the impact of hydrophobisation and basalt fibres (BF) addition on SFE, frost resistance, contact angle (CA), and roughness. A device capable of calculating all parameters was used to indicate the surface roughness and 3D topography. Prior to and after conducting surface and weight hydrophobisation, the contact angle of mortars was specified. Subsequently, it was used for carrying out SFE calculation by means of Neumann's method, enabling us to characterize the adhesion properties and wettability of mortars. The research indicated that the surface roughness was substantially decreased, in turn raising the frost resistance. The corrosion resistance drops when the surface roughness, water absorption, and number of fibres in the mortar increase. The SEM images presenting the structure of polysiloxane coating and mortars were provided.
\end{abstract}

Keywords: polysiloxanes; mortar; basalt fibre; roughness; surface free energy

\section{Introduction}

The basalt fibre (BF), developed in Moscow Research Institute of Glass and Plastic in 1953-1954, is a technologically advanced fibre $[1,2]$. While analyzing its chemical composition it can be observed that silica constitutes the main component (over $45 \%$ ), followed by $\mathrm{Al}_{2} \mathrm{O}_{3}$ (approximately $11 \%-13 \%$ ) [1-4]. The diameter of fibres usually ranges from 10 to $20 \mu \mathrm{m}$, whereas the length varies from 3 to $120 \mathrm{~mm}$ [1]. The production process of basalt fibres consumes less energy and is cheaper than in the case of glass or carbon fibres [5]; additionally, it is not hazardous or carcinogenic [6]. According to Hao L.C. and $\mathrm{Yu}$ W.D. the thermal stability of basalt fibres is superior to the one characterizing glass fibres [7]. On the other hand, Moiseev E.A. et al. noted that the crystallization behavior and presence of iron oxide in the production of basalt fibres have an impact on the heat temperature stability [8,9]. The literature studies indicate that basalt fibres are employed in mortars in order to reinforce cement matrices, owing to their properties including high durability, better stability, improved toughness, and ability to resist repeated impact [4]. The basalt fibres in mortars improve the flexural and tensile strength, ductility, as well as cracking energy of cement materials. The professional literature indicates that a sensible addition of basalt fibres amounts to approximately $1-3 \%(w / w)$ of binder [4]. The addition of basalt fibres to the mortar reduces the drying shrinkage and greatly improves the abrasion resistance and the resistance to frost and alkali $[10,11]$. The content of fibres frequently increases the porosity and absorptivity, which was indicated by the authors in another work [12]. The effect of porosity within the interfacial 
zone between layers on pull-off adhesion was described. by Sadowski Ł. et al. [13]. The morphology of concrete surface plays an important role in majority of classic physical phenomena, such as electric and thermal conductivity, adhesion, and wettability [14,15]. Sadowski Ł. and Mathia T.G. observed that the morphology of concrete surface depends on aggregate size, material strength, moisture and cleanliness of substrate, and preparation of the surface [14]. The corrosion of elements repaired with concrete is strongly dependent on the appropriate adhesion between layers [16,17].

The content of short basalt fibres causes changes in the topography and roughness of the surface, which is closely connected with the adhesion of these mortars to the substrate, as well as wettability of mortars and adhesion of paints or impregnating preparations. Theoretically, knowing the structure of a rough surface, it is possible to predict the general behaviour of a liquid on it. There are empirical theories pertaining to the wettability of rough surfaces. Usually, it is assumed that if $R_{a}<0.5 \mu \mathrm{m}$, the impact of roughness on the contact angle is insignificant [18]. Certain publications describe the physical mechanisms characterizing this phenomenon [19]. Wenzel [20] noted that a rough surface with hydrophobic film behaved as if it was more hydrophobic in certain spots and more hydrophilic in other. Wenzel also suggests that the surface geometry has a significant impact on the static contact angle [20]. Bico et al. [21] indicated the influence of concrete surface geometry on the adhesion of repair systems. The obtained results indicate that in the case of surface roughness, the occurrence of cracks and loose concrete chunks are important factors that have an impact on the adhesion of repair systems, similarly to, e.g., hydrophobisation.

One of the methods of protecting wettable mortar surfaces is the impregnation of porous construction materials [22-24]. Organosilicon compounds are frequently used for the hydrophobisation of construction materials. Silanes and siloxanes are considered one of the most efficient and safest hydrophobising agents. Alkyl potassium silicates, alkoxysilanes, hydrated siloxanes, and siloxanes in hydroxide form are used as silicone hydrophobisers. Alkyl potassium silicates are commercially available only as a highly alkaline $(\mathrm{pH}=14)$ aqueous solution. The remaining compounds are soluble only in organic solvents.

The studies indicated high hydrophobisation efficiency of mortars and lightweight concretes [22,25-27]. Silanes, siloxanes, and silicone resins do not differ in respect to the activity, but rather in terms of the molecule size and structure. The pore diameter and surface porosity indicate the possibility of adsorption of a compound and the critical size of a molecule [28]. Certain compounds, including molecular siliconates are characterized by large diameters, which prevent the penetration of the fine-pored structure of some materials, such as cement mortars. Because polysiloxanes agents are often used in the hydrophobisation of building materials, a preparation with large molecules was applied in our research. The chemical composition of monomers is the main factor governing the hydrophobisation efficiency. The alkyl groups (R) regulate the concrete hydrophobisation efficiency, while the alkoxyl groups (OR') determine the compound reactivity. The type of solvent, i.e., a carrier, is a decisive parameter when it comes to choosing appropriate preparation. The applicable solvents include alcohol, white sprit, and water. However, the alcohol-based preparations do not guarantee achieving the required hydrophobisation efficiency. Therefore, they are not used in civil engineering [12,29].

To the best of our knowledge, basalt-reinforced hydrophobised cement mortars have not been tested in terms of roughness and its influence on wettability, surface free energy (SFE), and frost corrosion. Therefore, determining the relation between interphase roughness of cement mortars as well as wettability and SFE seems necessary because the roughness of cement mortars has a great impact on the type of damage [29]. The physical condition of thin siloxane film in capillaries of porous materials was investigated and analyzed on the basis of mortars reinforced with basalt fibres. The research performed on the surface of cement mortars aimed to indicate a diversified geometrical structure of the considered cement mortars containing basalt fibres, taking into account their surface free energy and frost resistance following hydrophobisation carried out by means of a methyl silicone resin in potassium hydroxide. 


\section{Materials and Methods}

The presented research consisted of three stages of examinations: characterisation of cement mortars and water repellent agents, description of the sample preparation and test methods, and determination of the parameters of hydrophobised mortars.

\subsection{Characteristics of Materials}

Two series were prepared, each comprising four mortars with BF. Their compositions per $\left(\mathrm{kg} \cdot \mathrm{m}^{-3}\right)$ are presented in Table 1.

Table 1. Composition of the mortars with basalt fibres.

\begin{tabular}{cccccccccc}
\hline \multirow{2}{*}{ Materials } & \multirow{2}{*}{ Unit } & \multicolumn{4}{c}{ Series 1 } & \multicolumn{4}{c}{ Series 2 } \\
\cline { 3 - 10 } & & M0 & M0.5 & M1 & M1.5 & H0 & H0.5 & H1 & H1.5 \\
\hline Portland cement I 32.5R & $\left(\mathrm{kg} \cdot \mathrm{m}^{-3}\right)$ & 280 & 280 & 280 & 280 & 274.4 & 274.4 & 274.4 & 274.4 \\
Quartz sand $<2 \mathrm{~mm}$ & $\left(\mathrm{~kg} \cdot \mathrm{m}^{-3}\right)$ & 1483 & 1483 & 1483 & 1483 & 1483 & 1483 & 1483 & 1483 \\
Water & $\left(\mathrm{kg} \cdot \mathrm{m}^{-3}\right)$ & 126 & 126 & 126 & 126 & 126 & 126 & 126 & 126 \\
Hydrophobic admixture & $\left(\mathrm{kg} \cdot \mathrm{m}^{-3}\right)$ & - & - & - & - & 5.49 & 5.49 & 5.49 & 5.49 \\
Basalt fiber 12-18 $\mu \mathrm{m}$ & $\left(\mathrm{kg} \cdot \mathrm{m}^{-3}\right)$ & - & 13.2 & 26.4 & 39.6 & - & 13.2 & 26.4 & 39.6 \\
Basalt fiber 12-18 $\mu \mathrm{m}$ & $(\%)$ & 0 & 0.5 & 1 & 1.5 & 0 & 0.5 & 1 & 1.5 \\
\hline
\end{tabular}

Series 1 involves the reference mortars (M0-M1.5) without hydrophobising agent addition, whereas Series 2 (H0-H1.5) includes an addition of cementitious waterproofing material—siloxane. The surface-hydrophobised samples were designated as MR (resin hydrophobised series 1-M) and HR (resin hydrophobised series $2-\mathrm{H}$ ). The addition was dosed in the amount of $2 \%$ in relation to cement mass. Mortars in each series differed in respect to the BF content. The quantity of BF varied in percentage: $0 ; 0.5 ; 1 ; 1.5 \%$. The water/cement $(w / c)$ ratio equal to 0.45 was assumed. The technical parameters of applied Portland cement CEM I 32.5R are presented in Table 2.

Table 2. Technical parameters of Portland cement CEM I 32.5R [22,29].

\begin{tabular}{cccccc}
\hline Parameters & Unit & Value & Parameters & Unit & Value \\
\hline Specific surface & $\left(\mathrm{cm}^{2} \cdot \mathrm{g}^{-1}\right)$ & 3985 & Density & $\left(\mathrm{g} \cdot \mathrm{cm}^{-3}\right)$ & 3.05 \\
Initial setting time & $(\mathrm{min})$ & 248 & Volume stability & $(\mathrm{mm})$ & $<10$ \\
Loss on ignition by weight cement & $(\%)$ & 5.0 & $\mathrm{SO}_{3}$ content & $(\%)$ & 2.798 \\
Compressive strength & $(\mathrm{MPa})$ & & $\mathrm{Cl}$ content & $(\%)$ & 0.066 \\
after 2 days & & 17.6 & $\mathrm{Cr}(\mathrm{VI})$ diss. content & $(\mathrm{ppm})$ & 0.26 \\
after 28 days & & 43.2 & $\mathrm{Na}_{2} \mathrm{O}_{\text {eq }}$ content & $(\%)$ & 0.78 \\
\hline
\end{tabular}

The characteristics of applied BF are presented in Table $3[2,3,30]$. The employed loose fibres and fibres in mortars were shown in SEM images in Figure 1.

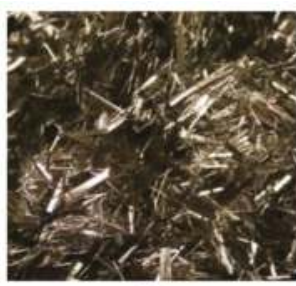

(a)

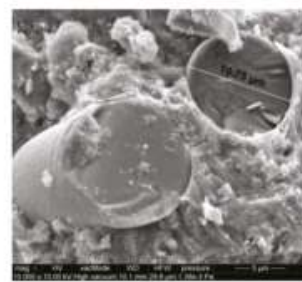

(b)

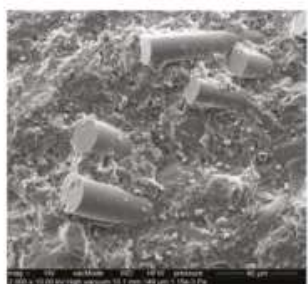

(c)

Figure 1. Basalt fibres: (a) loose, (b) in mortar $\times 10,000$, (c) in mortar $\times 2000$. 
Table 3. Parameters of basalt fibres [2,3,30].

\begin{tabular}{ccc}
\hline Parameters & Unit & Value \\
\hline Hardness on the Mosh' scale & $(-)$ & 8.5 \\
Elongation to fracture & $(\%)$ & $2.4-3.1$ \\
Softening temperature & $\left({ }^{\circ} \mathrm{C}\right)$ & 960 \\
Modulus of elasticity & $(\mathrm{GPa})$ & $89-110$ \\
Tensile strength & $(\mathrm{MPa})$ & $2800-4500$ \\
Thermal conductivity & $\left(\mathrm{W} \cdot \mathrm{m}^{-1} \cdot \mathrm{K}^{-1}\right)$ & 1.67 \\
Moisture absorption & $(\%)$ & $<0.1$ \\
Coefficient of linear thermal expansion & $\left(\mathrm{K}^{-1}\right)$ & $5.5 \times 10^{-7}$ \\
Specific heat capacity & $\left(\mathrm{kJ} \cdot \mathrm{kg}^{-1} \cdot \mathrm{K}^{-1}\right)$ & 0.86 \\
Constant operating temperature & $\left({ }^{\circ} \mathrm{C}\right)$ & 680 \\
Melting temperature & $\left({ }^{\circ} \mathrm{C}\right)$ & 1450 \\
Operating temperature range & $\left({ }^{\circ} \mathrm{C}\right)$ & -260 to +750 \\
\hline
\end{tabular}

The utilized basalt fibres were about $24 \mathrm{~mm}$ in length, with low diameter of $11-18 \mu \mathrm{m}$. The fibres were characterized by rough and irregular surface. The constituent minerals of the considered igneous basalt rock include plagioclase: $\mathrm{Na}\left(\mathrm{AlSi}_{3} \mathrm{O}_{8}\right)-\mathrm{Ca}\left(\mathrm{Al}_{2} \mathrm{SiO}_{8}\right)$; pyroxene: $\mathrm{XY}_{2}\left[\left(\mathrm{Si}, \mathrm{Al}_{2} \mathrm{O}_{6}\right]\right.$ (where $\mathrm{X}-\mathrm{Ca}, \mathrm{Mg}, \mathrm{Fe}^{2+}$ and $\mathrm{Y}$ stands for $\mathrm{Fe}^{3+}, \mathrm{Al}$, $\mathrm{Ti}$ ); and olivine: $(\mathrm{Fe}, \mathrm{Mg})_{2} \mathrm{SiO}_{4}$, which was proven by Kamiya S. et al. [31] in a US Patent.

The classification of waterproofing preparations was carried out on the basis of the authors' own studies [22,28,32]. Mineral waterproofing grout-siloxane was used in the Series 2. It is a highly cementitious waterproofing admixture of siloxane for mortars with the following properties: strong adhesion to the substrate, water impermeable, resistant to water and frost, resistant to the effects of mechanical and chemical loads. The characteristic data of the product are described in Table 4 .

Table 4. Technical parameters of mineral waterproofing grout [32].

\begin{tabular}{ccc}
\hline Parameters & Unit & Value \\
\hline Water absorption coefficient $w_{24}$ & $\left(\mathrm{~kg} \cdot \mathrm{m}^{-2} \cdot \mathrm{h}^{-0.5}\right)$ & $<0.1$ \\
Water requirements & $(\%)$ & $20-21$ \\
Water vapour diffusion $\delta$ & $\left(\mathrm{g} \cdot \mathrm{m}^{-1} \cdot \mathrm{h}^{-1} \cdot \mathrm{hPa}^{-1}\right)$ & $<200$ \\
Compressive strength after 28 days & $(\mathrm{MPa})$ & 30 \\
Tensile bending strength after 28 days & $(\mathrm{MPa})$ & 6 \\
Working time & $(\mathrm{min})$ & 60 \\
\hline
\end{tabular}

The technical parameters of the applied surface and water hydrophobisation agent are presented in Table 5. The surface hydrophobisation was conducted using macromolecular polysiloxane in potassium hydroxide in an aqueous solvent: $\mathrm{R}-\mathrm{O}-\mathrm{Si}-[\mathrm{O}-\mathrm{Si}]_{\mathrm{n}}-\mathrm{O}-\mathrm{Si}-\mathrm{R}$, where: $\mathrm{R}-$ methyl derivative.

Table 5. Technical parameters of polysiloxane and water.

\begin{tabular}{lccc}
\hline Parameters & Unit & Polysiloxane & Water \\
\hline Density & $\left(\mathrm{g} \cdot \mathrm{cm}^{-3}\right)$ & 1.03 & 0.99 \\
Viscosity $\eta$ & $(\mathrm{Pa} \cdot \mathrm{s})$ & $0.98 \times 10^{-3}$ & $0.89 \times 10^{-3}$ \\
Surface tension $\gamma$ & $\left(\mathrm{N} \cdot \mathrm{m}^{-1}\right)$ & $77.24 \times 10^{-3}$ & $72 \times 10^{-3}$ \\
Surface tension-to-viscosity ratio $\eta / \gamma$ & $(-)$ & 78.82 & 80.90 \\
\hline
\end{tabular}

\subsection{Methods}

The studies on CEM I 32.5R were conducted in accordance with PN-EN 197-1:2012 standard [33]. Samples with the dimensions of $40 \mathrm{~mm} \times 40 \mathrm{~mm} \times 160 \mathrm{~mm}$ were prepared on the basis of EN 196-1:2016-07 standard [34]. All components, except for basalt fibres, were first mixed for two minutes. 
Afterward, BF were added. Following another two minutes, water was added and mixed for subsequent two minutes. Moulds were filled to the half of their capacity and compacted for one minute on a flow table. Afterward, a second layer of mortars was laid, and the samples were compacted again. The samples were stored under laboratory conditions for $24 \mathrm{~h}$ and then removed from the mould and stored in a climatic chamber at the temperature of $23.5^{\circ} \mathrm{C}$ and relative humidity of $73.5 \%$ for 28 days.

Investigations pertaining to density, as well as volumetric and apparent densities, and open porosity of mortars were carried out in line with PN-EN 1015-10:2001 standard [35]. The research involved six samples with the dimensions of $40 \mathrm{~mm} \times 40 \mathrm{~mm} \times 160 \mathrm{~mm}$. Prior to the investigation, the samples were dried to a constant mass. The flexural strength test was carried out 28 days after moulding, on the basis of EN 1015-11:2001 [36] on three samples of each mortar. The compression strength test was performed on halves of the mortar blocks obtained in the course of flexural strength test (6 samples). The samples were dried at the temperature of $65^{\circ} \mathrm{C}$ to a constant weight, weighed, and hydrophobised (by applying the preparation twice using a brush) with the preparation of polysiloxane in potassium hydroxide. The preparation-water ratio was 1:8. Before other investigations were carried out, the samples were seasoned for 7 days under laboratory conditions in order to enable the hydrolytic polycondensation to occur, yielding polysiloxane gel in the subsurface zone of mortars.

The efficiency of hydrophobisation and its influence on the durability of mortars with basalt fibres was evaluated afterwards. Absorptivity of mortars was indicated in accordance with BS 1881-122:2011 standard [37] and EN ISO 7783:2012 standard [38]. The samples were studied in 1 and 14-day intervals in order to determine the impact of moisture on the hydrophobized mortars. The resistance to freezing-thawing (F-T) cycles was determined on the basis of PN-EN 12012:2007 standard [39]. The experiment involved the application of six samples for each batch of mortar with the dimensions $40 \mathrm{~mm} \times 40 \mathrm{~mm} \times 160 \mathrm{~mm}$. The samples were periodically frozen at the temperature of $-18 \pm 2{ }^{\circ} \mathrm{C}$ for at least $4 \mathrm{~h}$ and then thawed in water at the temperature of $18 \pm 2{ }^{\circ} \mathrm{C}$ for a period between two and four hours. Each freeze-thaw (F-T) process was a single experiment cycle. Fifty cycles were conducted instead of the required 25 to prove the increased frost resistance of the examined mortars. After the final cycle was finished, the samples were dried to a constant weight and weighed again to measure the mass lost after determining the frost resistance. The compression strength test of mortars was performed as well.

Determination of surface roughness and 3D topography was conducted in a T8000 RC120-400 device by JENOPTIC, Jena, Germany, similarly to the procedure described in Barnat-Hunek et al. [29]. The measurements were performed using unified GUI (graphical user interface), enabling to calculate all parameters of considered roughness and undulation profiles, as well as evaluation of the geometric characteristics both before and after conducting the surface hydrophobisation. The device has a vertical measuring range of $60 \mathrm{~mm}$ and resolution of $50 \mathrm{~nm}$, as well as wavecontour ${ }^{\mathrm{TM}}$ digital probing system with digital linear scales in the $\mathrm{Z}$ and $\mathrm{X}$-axes. Roughness was measured on inclined or bent surfaces with the resolution of $6 \mathrm{~nm}$ in the measurement range of $6 \mathrm{~mm}$. Roughness and contour are evaluated by two separate probing systems. Ten randomly selected fragments of mortar samples were examined, measuring the porosity of a $3.5 \times 3.5 \mathrm{~mm}^{2}$ area. The measurement time of a single area approximated two hours. The images of surface structure and profilograms obtained directly by using a 3D profilographometer reflected the obtained roughness parameters characterizing the surface of studied mortars reinforced with basalt fibres.

The mortars containing basalt fibres have quite uneven surface, which may be characterized by the following parameters $[29,40]$ :

- $\quad R_{\mathrm{a}}$-Average Roughness defined as the average deviation of the profile in relation to its mean line and parameter more sensitive to peaks and valleys;

- $\quad R_{\mathrm{pm}}$-Mean Peak Height defined as the mean peak height from each length of sampling;

- $\quad R_{\mathrm{vm}}-$ Mean Valley Depth as the mean maximum value of valley depth for each length of sampling;

- $\quad R_{\mathrm{p}}$-Maximum Peak Height as the maximum height of peak within evaluation length;

- $R_{\mathrm{V}}$-Maximum Valley Depth as the maximum depth observed within the evaluation length; 
- $\quad R_{\max }$-Maximum Peak-to-Valley Height understood as the maximum peak-to-valley height within any of the sampling lengths; $R_{\max }=R_{\mathrm{V}}+R_{\mathrm{p}}$.

The contact angle (CA) characterizing the liquid drop was measured on a research stand, comprising a goniometer and a camera used for capturing the image of a drop put onto surface of a sample. The analysis of the contact angle was conducted with distilled water. Wetting angles $\theta_{w}$, which correspond to the surface coatings, were measured with a liquid characterized by known total SFE values $\left(\gamma_{\mathrm{w}}\right)$. The constant volumes of liquid drops approximated $2 \mathrm{~mm}^{3}$ and were applied onto the sample surface by means of a micropipette. The surfaces of samples were corrected smoothed to mitigate the impact of surface roughness. Five drops were applied onto each sample, given the heterogeneity of the material. The measurements were conducted at the temperature approximating $22.5^{\circ} \mathrm{C}$ at the moment of applying a drop (Figure 2).

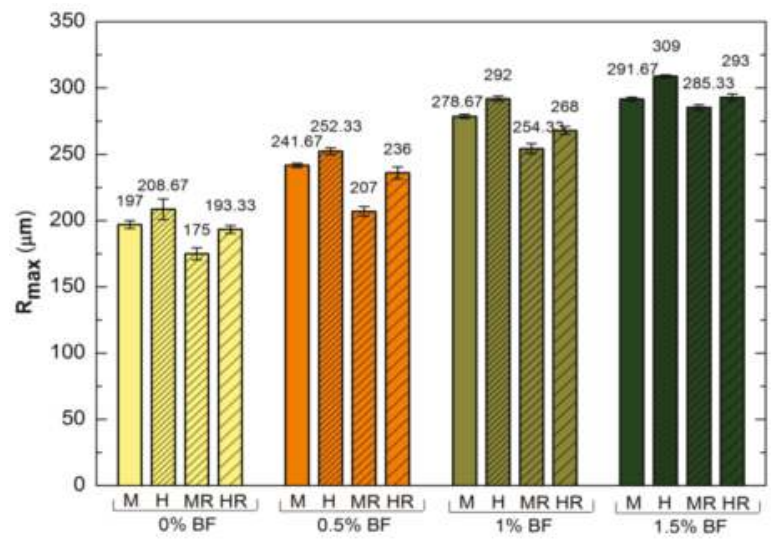

Figure 2. Maximum Peak-to-Valley Height $\left(R_{\max }\right)$ of basalt fibres-reinforced cement mortars before and after hydrophobisation with polysiloxanes.

The Neumann model, which constitutes one of the most common methods of calculating SFE, was used in order to determine this parameter [12,22]. The employed equation was as follows [41]:

$$
\cos \theta_{\mathrm{w}}=\left[e^{-0.000125\left(\gamma_{\mathrm{s}}-\gamma_{w}\right)^{2}} 2 \sqrt{\frac{\gamma_{\mathrm{s}}}{\gamma_{\mathrm{w}}}}-1\right]
$$

where: $\gamma_{\mathrm{S}}$-total SFE, $\gamma_{\mathrm{w}}-\mathrm{SFE}$ of water $=72.8\left(\mathrm{~mJ} \cdot \mathrm{mm}^{-2}\right), \theta \mathrm{w}$ - water contact angle.

Scanning electron microscopy (SEM) (Quanta FEG 250 microscope by FEI, Hillsboro, OR, USA) was employed to determine the morphology, structure of mortars, distribution of polysiloxane gel, and the interfacial transition zone between the cement paste and BF. The samples for SEM studies were glued to a carbon holder by means of carbon glue. Afterwards, they were covered with a $50 \mathrm{~nm}$ thick layer of carbon in a coating machine in order to achieve conductivity on the surface of sample.

\section{Results}

\subsection{Properties of Basalt Fibres-Reinforced Cement Mortar}

Table 6 presents both the mechanical and physical properties characterizing the considered mortars prior to being hydrophobized. 
Table 6. Technical parameters of non-hydrophobised basalt fibres-reinforced cement mortar.

\begin{tabular}{lccccccccc}
\hline \multirow{2}{*}{ Parameters } & \multirow{2}{*}{ Unit } & \multicolumn{4}{c}{ Series 1 } & \multicolumn{4}{c}{ Series 2 } \\
\cline { 3 - 10 } & & M0 & M0.5 & M1 & M1.5 & H0 & H0.5 & H1 & H1.5 \\
\hline Apparent density $\rho_{\mathrm{a}}$ & $\left(\mathrm{kg} \cdot \mathrm{m}^{-3}\right)$ & 2020 & 1956 & 1941 & 1823 & 1952 & 1892 & 1823 & 1741 \\
Density $\rho$ & $\left(\mathrm{kg} \cdot \mathrm{m}^{-3}\right)$ & 2320 & 2301 & 2287 & 2245 & 2300 & 2265 & 2230 & 2207 \\
Total porosity $P$ & $(\%)$ & 12.9 & 14.9 & 15.2 & 18.7 & 15.1 & 16.5 & 18.3 & 21.1 \\
Open porosity $P_{\mathrm{o}}$ & $(\%)$ & 5.3 & 7.5 & 9.2 & 11.4 & 8.8 & 9.9 & 10.4 & 12.8 \\
Compressive strength $f_{\mathrm{c}}$ & $(\mathrm{MPa})$ & 9.53 & 9.01 & 8.31 & 7.63 & 7.23 & 7.06 & 6.41 & 5.68 \\
Flexural tensile strength $f_{\mathrm{m}}$ & $(\mathrm{MPa})$ & 6.10 & 6.74 & 7.87 & 8.96 & 5.32 & 5.98 & 6.47 & 7.06 \\
\hline
\end{tabular}

\subsection{Effectiveness of Hydrophobisation of Basalt Fibres-Reinforced Cement Mortar}

\subsubsection{Roughness and Microstructure of Mortars}

The characteristics of roughness obtained for the tested mortars are presented in Table 7.

Table 7. Roughness characteristics of basalt fibres-reinforced cement mortars.

\begin{tabular}{cccccccc}
\hline \multirow{2}{*}{ Type of mortars } & \multicolumn{6}{c}{ Mortar roughness characteristics $(\mu \mathrm{m})$} \\
\cline { 3 - 8 } & $\boldsymbol{R}_{\mathbf{a}}$ & $\boldsymbol{R}_{\mathbf{v m}}$ & $\boldsymbol{R}_{\mathbf{p m}}$ & $\boldsymbol{R}_{\mathbf{p}}$ & $\boldsymbol{R}_{\mathbf{v}}$ & $\boldsymbol{R}_{\max }$ \\
\hline \multirow{4}{*}{ Series 1 } & M0 & 13.3 & 102 & 42 & 52 & 145 & 197 \\
& M1 & 16.2 & 140 & 70 & 78.7 & 163 & 241.7 \\
& M1.5 & 18.4 & 167 & 75 & 90 & 188.7 & 278.7 \\
& H0 & 19.3 & 179 & 84 & 95.7 & 196 & 291.7 \\
\hline \multirow{4}{*}{ Series 2 } & H0.5 & 19.3 & 111 & 50 & 58.7 & 151 & 208.7 \\
& H1 & 22.9 & 179 & 82 & 99 & 193 & 292 \\
& H1.5 & 25.7 & 193 & 92 & 108 & 201 & 309 \\
\hline
\end{tabular}

Figure 2 presents the changes of mortar $R_{\max }$ with reactive siloxane (Series 2 ) and the changes following the surface hydrophobisation with polysiloxane in potassium hydroxide.

Microroughness and the representative profilograms showing the surface of M1 basalt fibres-reinforced cement mortars-before and after surface modification with polysiloxanes-are presented in Figure 3 a,b.

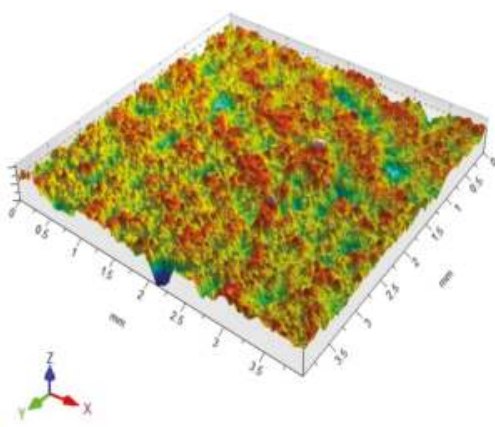

(a)

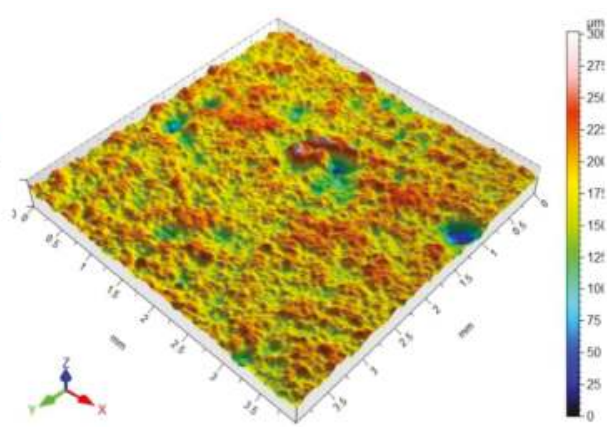

(b)

Figure 3. Microroughness and representative profilograms showing the surface of basalt fibres-reinforced cement mortars: (a) unmodified M1; (b) M1 with polysiloxane coating. 
Figure 4 presents the SEM images showing the surface of basalt fibres-reinforced cement mortars before and after hydrophobisation.

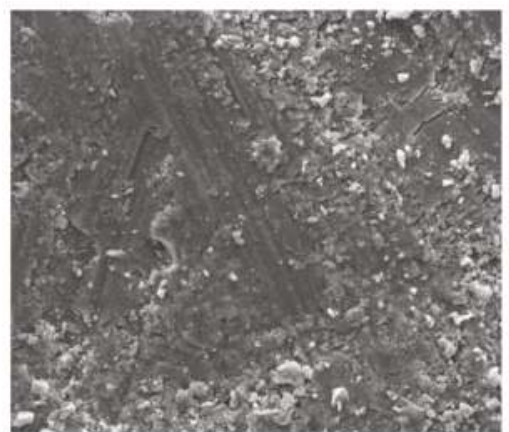

(a) M0-unmodified

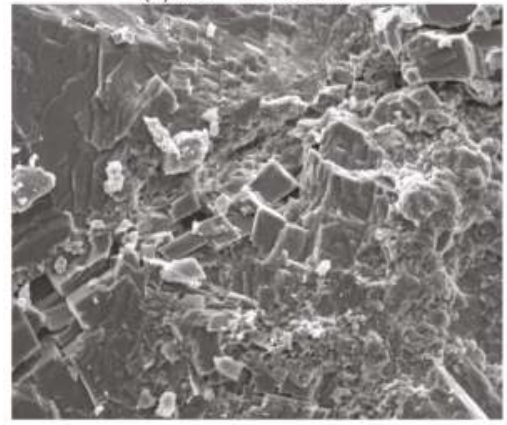

(c) M1-unmodified

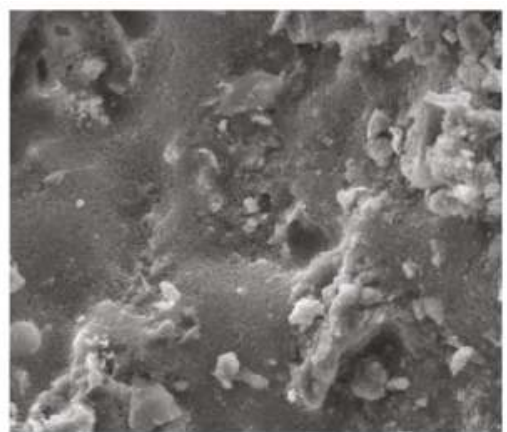

(b) MOR-with polisiloxane coating

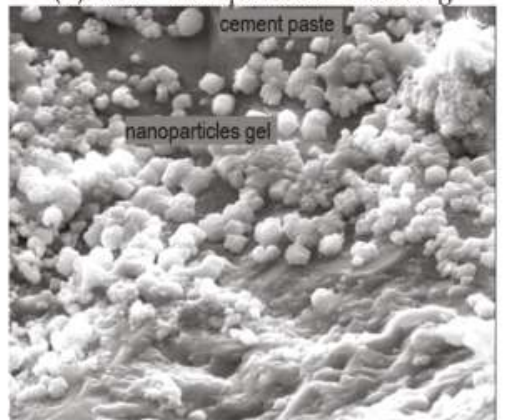

(d) M1R-with polisiloxane coating

Figure 4. Surface microstructure of unmodified basalt fibres-reinforced cement mortars and the mortars with polysiloxane coating $(\times 4000)$.

Figure 5 presents the microstructure and adhesion of cement paste to BF.

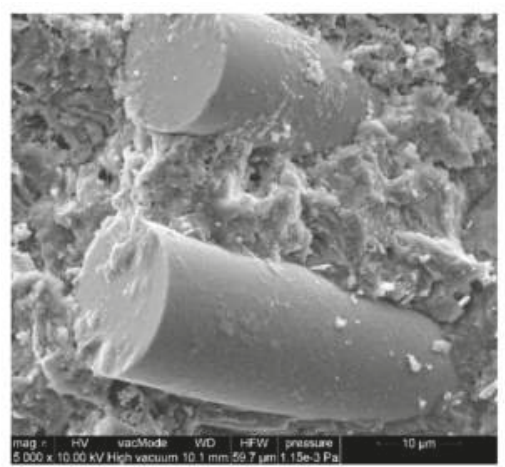

(a) M1 ( 5000$)$

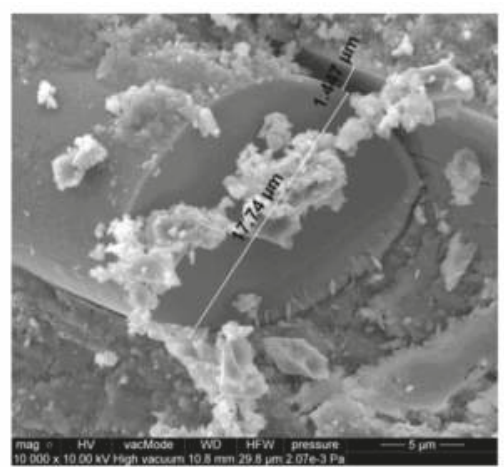

(b) $\mathrm{H} 1(\times 10000)$

Figure 5. Cont. 


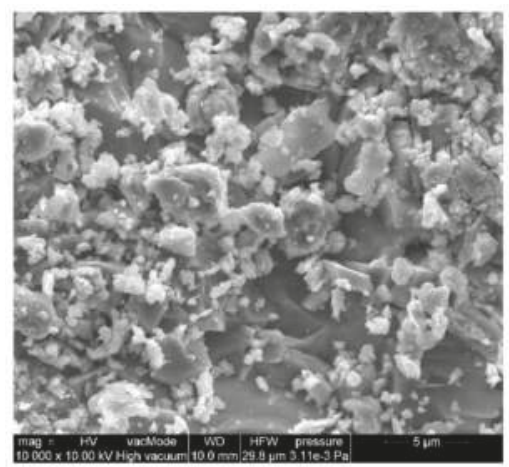

(c) $\mathrm{M} 1(\times 10,000)$

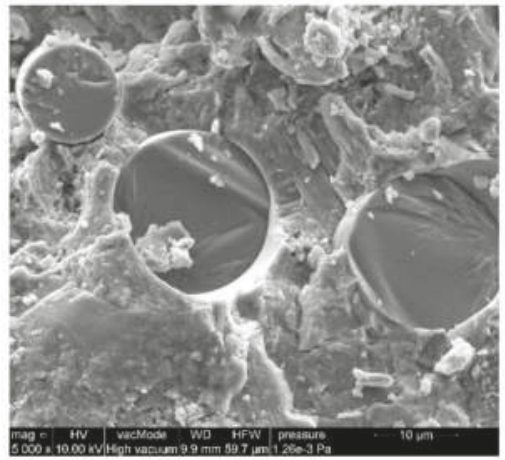

(e) M1 ( $\times 5000)$

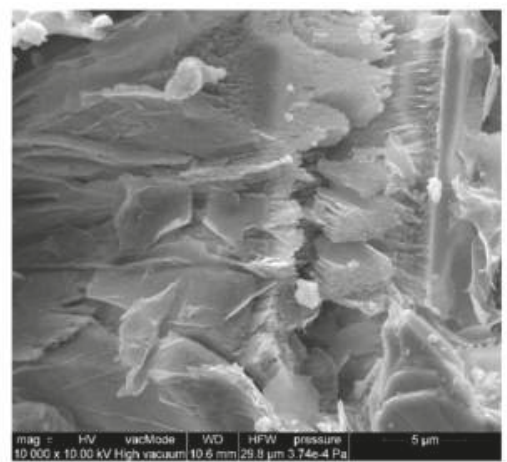

(d) $\mathrm{H} 1(\times 10,000)$

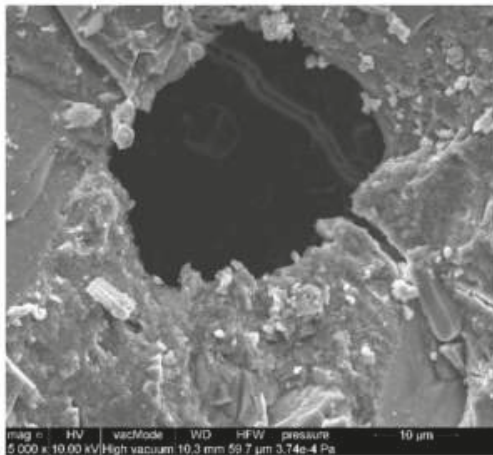

(f) $\mathrm{H} 1(\times 5000)$

Figure 5. Internal microstructure of basalt fibres-reinforced cement mortars: unmodified (M1) and with siloxane admixture (H1).

\subsubsection{Absorptivity, Wettability, Surface Free Energy (SFE) of Mortars}

In order to show the impact of hydrophobisation on the changes in absorptivity in time, an experiment was conducted after one and 14 days. The results are presented in Figure 6.

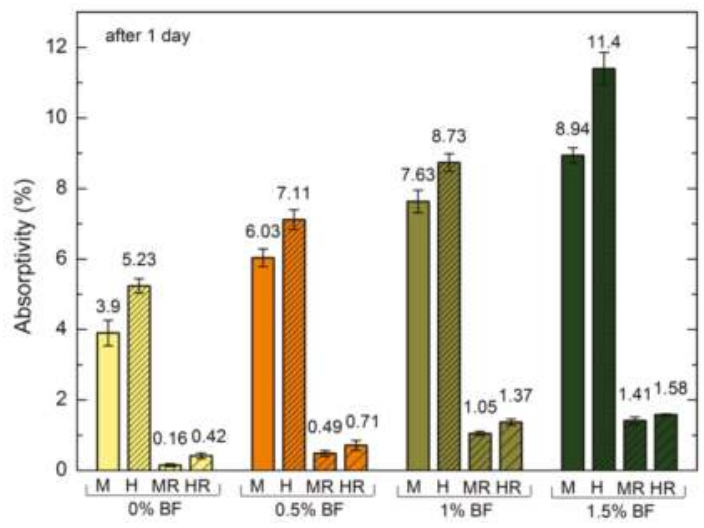

Figure 6. Cont. 


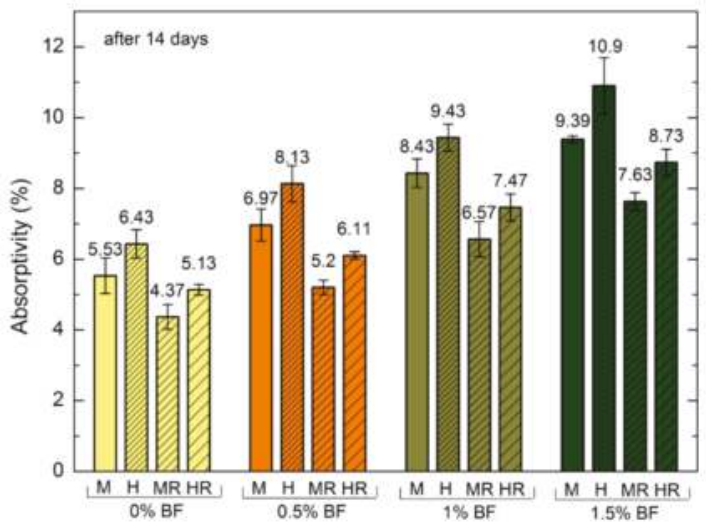

Figure 6. Absorptivity of hydrophobized and reference mortars with BF after one and 14 days.

Contact angle (CA) and SFE constitute other parameters defining the wettability of a material [22,29]. Figure 7 presents exemplary photos of CA water measurements of cement mortars. The SFE of all analyzed cement mortars was calculated by means of the Neumann's method on the basis of CA measurement. Table 8 presents the CA of water measured on each mortar and the SFE calculated on its basis.

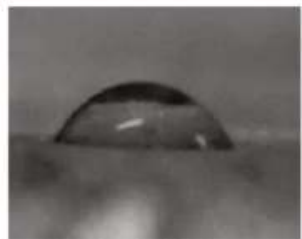

(a) $\mathrm{M} 0, \mathrm{CA}=52.2^{\circ}$

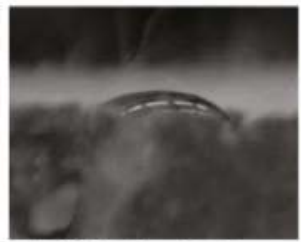

(b) $\mathrm{M} 1.5, \mathrm{CA}=39.4^{\circ}$

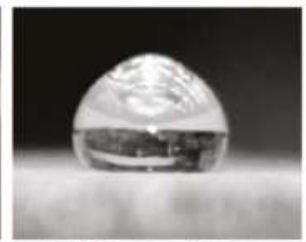

(c) $\mathrm{M} 0 \mathrm{R}, \mathrm{CA}=122.1^{\circ}$

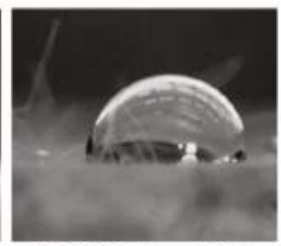

(d) $\mathrm{M} 1.5 \mathrm{R}, \mathrm{CA}=87.5^{\circ}$

Figure 7. Contact angle of a water drop in mortar with/without BF.

Table 8. Contact angle (CA) and surface free energy (SFE) of basalt fibres-reinforced cement mortars.

\begin{tabular}{ccccccccccc}
\hline \multirow{2}{*}{ Parameters } & \multirow{2}{*}{ Unit } & \multicolumn{4}{c}{ Series 1 } & \multicolumn{4}{c}{ Series 2 } \\
\cline { 3 - 10 } & & M0 & M0.5 & M1 & M1.5 & H0 & H0.5 & H1 & H1.5 \\
\hline CA before hydrophobisation & $\left({ }^{\circ}\right)$ & 52.2 & 48.2 & 41.5 & 39.4 & 47.2 & 40.7 & 39.1 & 30.4 \\
CA after hydrophobisation & $\left({ }^{\circ}\right)$ & 122.1 & 114.1 & 94.7 & 87.5 & 100.8 & 98.7 & 82.5 & 77.6 \\
SFE before hydrophobisation & $\left(\mathrm{mJ} \cdot \mathrm{m}^{-2}\right)$ & 52.49 & 54.81 & 58.56 & 59.70 & 55.38 & 59.0 & 59.86 & 64.29 \\
SFE after hydrophobisation & $\left(\mathrm{mJ} \cdot \mathrm{m}^{-2}\right)$ & 10.53 & 14.78 & 26.32 & 30.81 & 22.58 & 23.86 & 33.93 & 37.0 \\
\hline
\end{tabular}

\subsubsection{Frost Resistance of Mortars}

The test used for the evaluation of mortars durability involved investigation of the frost resistance. The condition of samples following a frost resistance test was presented in Figures 8 and 9. 


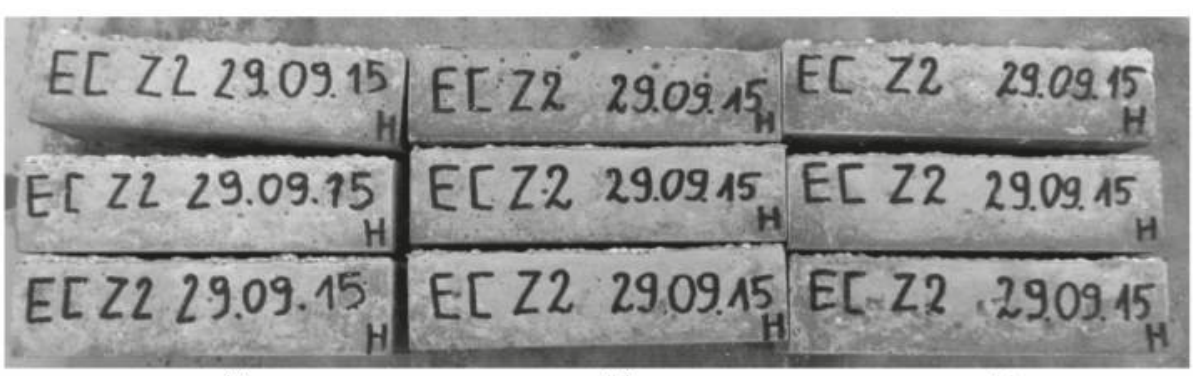

(a)

(b)

(c)

Figure 8. Condition of mortars Series 1 after 50 freeze-thaw (F-T) cycles: (a) M1; (b) M1R; (c) M0R.

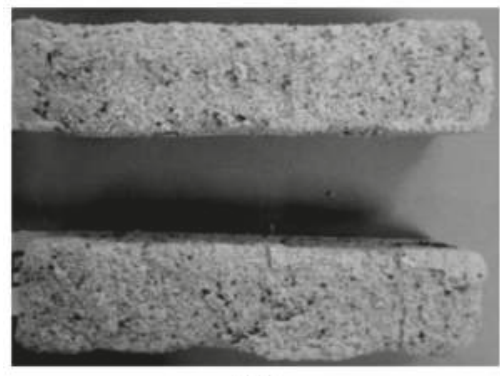

(a)

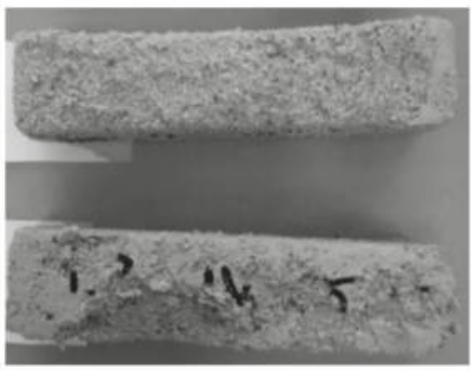

(b)

Figure 9. Condition of mortars Series 2 after 50 F-T cycles: (a) H0; (b) H1.5. Table 9

The mass loss and decrease in the compressive strength following 50 F-T cycles was shown in

Table 9. Mass loss and decrease in the compressive strength of basalt fibres-reinforced cement mortars after 50 F-T cycles.

\begin{tabular}{cccccccccc}
\hline \multirow{2}{*}{ Parameters } & \multirow{2}{*}{ Unit } & \multicolumn{4}{c}{ Series 1 } & \multicolumn{4}{c}{ Series 2 } \\
\cline { 3 - 10 } & & M0 & M0.5 & M1 & M1.5 & H0 & H0.5 & H1 & H1.5 \\
\hline Mass loss before hydrophobisation & $(\%)$ & 0.3 & 0.5 & 0.6 & 0.8 & 2.1 & 2.8 & 5.3 & 8.3 \\
\hline Mass loss after hydrophobisation & $(\%)$ & 0.01 & 0.1 & 0.2 & 0.24 & 0.7 & 1.2 & 2.4 & 2.7 \\
\hline $\begin{array}{c}\text { Decrease the compressive strength } \\
\text { before hydrophobisation }\end{array}$ & $(\%)$ & - & 1.1 & 2.3 & 2.5 & 4.3 & 6.7 & 13.2 & 24.3 \\
\hline $\begin{array}{c}\text { Decrease the compressive strength } \\
\text { after hydrophobisation }\end{array}$ & $(\%)$ & - & 0.4 & 0.9 & 1.1 & 2.1 & 3.2 & 4.5 & 5.2 \\
\hline
\end{tabular}

\section{Discussion}

\subsection{Properties of Basalt Fibres-Reinforced Cement Mortar}

Depending on the content of basalt fibres and hydrophobising admixture, mortars are characterized by diversified apparent density ranging from $1741 \mathrm{~kg} \cdot \mathrm{m}^{-3}$ for the $\mathrm{H} 1.5$ mortar with $1.5 \%$ basalt fibres and hydrophobising admixture content to $2020 \mathrm{~kg} \cdot \mathrm{m}^{-3}$ in the case of the standard cement mortar (M0). Porosity varies from 12.9 to $21.1 \%$. The highest porosity was observed in the mortar with hydrophobising admixture and the highest amount of analyzed fibres (H1.5). The drop in 
the compressive strength resulting from mineral waterproofing grout addition amounts to $24.1 \%$ for M0 and 25.5\% for M1.5. When 1.5\% basalt fibres addition was applied, the flexural strength increased by $32 \%$ in Series 1 and $24.6 \%$ in Series 2 . The presence of basalt fibres caused a great (39\%) rise of porosity. The smaller impact of the basalt fibres amount in a mortar can be observed in the results of strength tests. The M0 mortar without fibres or admixture exhibits the highest compressive strength. It can be observed that the strength is determined by the amount of fibres, but mainly by the content of mineral waterproofing grout in the mixtures. Similar conclusions can be formulated while analyzing the results obtained from the examination of physical properties. The strength of samples drops to the value as low as $26 \%$, when mineral waterproofing grout is utilized in mixtures. A similar impact of mineral hydrophobising admixture was confirmed by authors in the studies on other lightweight cement mortars [32]. A grain of cement is covered with polymer film, which hampers or prevents cement hydration, which was described by Łukowski P. [42]. Hence, the decrease in the compressive strength occured. The $5 \% w / w$ admixture to the cement is insufficient to create separate continuous phase in the concrete mixture. In principle, polymer should form a continuous film in the cement binder. The polymer admixture causes the formation of chemical bonds between particular concrete components, having an influence on the changes in microstructure as well as the changes in strength parameters. Unfavourable factors include the bilateral disruption of the polysiloxane gel hardening process in the aqueous environment of cement slurry and hydration of cement in the presence of polymer compound. The presence of water weakens the resin hardening process and thus the final polymer cross-linking reaction forming a film of polysiloxane gel is disrupted. It is likely that this phenomenon occurred with Series 2 cement mortars. In the case of the siloxane admixture utilized in the studies, it had an undesirable influence on the strength of mortars. As far as the lightweight cement mortars with expanded clay aggregate, perlite or zeolite are concerned, the active polycondensation reaction has occurred, forming cross-linked resin which may reinforce the material, which was shown by Barnat-Hunek D. [43]. The porous interfacial zone between the slurry and aggregate was sealed by polymers, which was confirmed in the research conducted by Czarnecki L. and Łukowski P. [44]. Thus, the adhesion of the slurry to aggregate is improved in the contact zone, thus increasing the strength of the entire material, as proven by Shaker F. et al. [45].

Following the analysis involving estimation of the pore structure parameters of the composite using Mercury intrusion porosimeter, thermogravimetric analysis and strength tests, Palou et al. [46] stated that the porosity by crystallization pressure could be enhanced in hardened pastes, which reduce the compression strength. Due to a drop in the strength, Palou et al. recommended that the share of fibres in a mortar should be within the approximate range of $0.1 \%-1.5 \%$ of cement weight [46]. Quattrociocchi et al. [47], who added 1, 3, 6\% BF to cement mortars, reported that the dispersion of fibres proved to be more difficult as their content increased in the mixture, which in turn reduced its workability. Therefore, it was necessary to add more water to the mixture, causing a potential increase in porosity. This significant reduction in liquidity was caused by the formation of spatial cross-linked structure due to a random distribution of fibres mixed with the mortar, which was proven in the research carried out by Ma et al. [48].

In our paper, BF improved the flexural strength by about 30\%. Dong et al. [49] indicated that the thermal contraction in a cement mortar can be reduced through reinforcement with basalt fibres. The fibres not only increase the flexural strength, but also improve the shock resistance and resistance against thermal contraction cracking. Semenov et al. [50] report that flexural tensile strength increased by $55 \%$ in the case of $1 \%$ basalt fibres addition, in comparison with the reference mortar samples. Li et al. [51] stated that cement mortars with basalt fibres find practical application in repairs. They observed that $30 \%$ of maximum crack width of bent, repaired reinforced concrete elements can be controlled. 


\subsection{Effectiveness of Hydrophobisation of Basalt Fibres-Reinforced Cement Mortar}

\subsubsection{Roughness and Microstructure of Mortars}

The presented study on surface roughness enables to indicate differentiation in the geometrical structure of the surface of both hydrophobized as well as reference materials, in terms of mechanical adhesion. This structure may govern the infiltration of polysiloxanes into rough surface of cement mortars with BF and increase the share of mechanical abrasion, thus influencing the strength of adhesion bonds and the hydrophobisation efficiency.

The analyzed roughness parameters indicate that the content of basalt fibres caused an increase in the average roughness $R_{\mathrm{a}}$ as well as $R_{\mathrm{vm}}$ the mean maximum value of valley depth for each length of sampling, $R_{\mathrm{p}}$ the maximum height of peak within evaluation length and the maximum valley depth $-R_{\mathrm{v}}$. $R_{\max }$ is the highest for the mortar with the greatest basalt fibres content and is $32 \%$ higher than $R_{\max }$ of the reference mortar both in Series 1 and 2 .

Reactive polysiloxane utilized in Series 2 mixtures increased the average roughness $R_{a}$ by up to $31 \%$ for the standard mortars and by up $13 \%$ with the highest share of basalt fibres (H1.5). However, $\mathrm{Ra}$ is only a mean value, which does not indicate the largest deviations on the material surface. Therefore, the $R_{\max }$ values that indicate the highest amplitude being a sum of the largest peak and deepest valley, were analyzed $\left(R_{\mathrm{p}}+R_{\mathrm{v}}\right)$. The $R_{\max }$ parameter is highest after the application of reactive siloxane admixture for all analyzed cement mortars, regardless of the BF amount. Following surface hydrophobisation, roughness is reduced by $11 \%-16 \%$ in the case of non-modified cement mortars, and by $4 \%-6 \%$ for Series 2 mortars. The mortars with the highest amount of BF are characterized by the greatest roughness, which is $30 \%-32 \%$ higher than $R_{\max }$ of standard cement mortars. According to Hay et al., impregnation is hindered by a multitude of pores and voids on the material surface, in addition to high roughness. This is particularly apparent when a preparation with high surface tension and viscosity is used, subsequently depositing on the peaks found on a rough surface [52]. A border layer weakening adhesion is formed when bubbles of air become trapped under the thin film of hydrophobising agent [43]. It is likely that the interaction characterizing the substrate and molecules of polymer coating is weakened when roughness is excessive.

The obtained results pertaining to the surface roughness parameters of the considered cement mortars are reflected in the images of surface structure and profilograms, obtained directly by means of a 3D profilografometer. The representative surface profilograms (Figure 3) of basalt fibres-reinforced cement mortars present visible differences in the microroughness of cement mortars before and after the application of polysiloxane film. Large polysiloxane gel molecules partially fill the pores of rough surface of mortars with BF addition, which was clearly presented in SEM images (Figure 4b,d). In the case of MOR mortars without basalt fibres, a thick, tight film of siloxane gel was formed. This situation is undesirable, because higher hydrophobisation efficiency is obtained when a thin film of polysiloxanes is formed in the material structure. This film slightly fills the pores of porous material forming a thin hydrophobic layer, which is more resistant to damage, cracks, and scratches, resulting from, e.g., frost corrosion [22,29,43]. In the case of the cement mortars with basalt fibres (Figure 4d), crystallization of polysiloxane gel phase, which loosely covers the surface of cement mortars, was clearly observed. Due to increased porosity, a thick, tight film was not formed.

Figure 5a,b,e depicts the adhesion of cement paste to BF. It can be observed that in the case of the mortar without siloxane admixture, the adhesion of slurry to fibres is very good. C-S-H phases have clearly crystallized (Figure 5a,e). In $\mathrm{H} 1$ mortar with hydrophobising admixture, characterized by higher porosity, $1.5 \mu \mathrm{m}$-wide cracks appear between fibres and slurry. Loose contact zone between BF and slurry causes a reduction in compression strength, which was described in Section 4.1.

According to [46], in the earlier period of hydrothermal hardening, hydrated products such as $\mathrm{C}-\mathrm{S}-\mathrm{H}$ were formed in the course of a chemical process. Due to the higher crystallization pressure than in the case of non-modified mortars, the porosity of cement mortars increases (Figure 5f), simultaneously reducing the compression strength. According to Palou et al. [46], a partial thermal 
decomposition of $\mathrm{C}_{3} \mathrm{AH}_{6}$ to $\mathrm{C}_{3} \mathrm{AH}_{1.5}$ occurs under high pressure in the later stage of the hardening process, resulting in the saturation of a sample with carbon dioxide. Additionally, the content of siloxane admixture slows down the cement hydration, affecting the quality and type of produced phases, which is illustrated, e.g., in Figure $5 c$,d. Figure $5 c$ presents the C-S-H phases and crystallized ettringite. On the other hand, there are no visibly crystallized ettringite lumps in Figure 5 because they are only found in insignificant amounts. The figure shows the lamellar rather than spherical structure of phases, in contrast to typical cement mortar. This indicates that both BF and siloxane addition caused changes in the structure of mortars, which affects their physical and strength parameters, e.g., high porosity, as shown in Figure $5 f$.

\subsubsection{Absorptivity, Wettability, SFE of Mortars}

The highest absorptivity is exhibited by H1.5 (Figure 6) mortar with siloxane admixture which has the highest porosity. In the first day of the experiment, the influence of surface hydrophobisation with polysiloxanes is the most evident. The absorptivity of standard mortars with this preparation was $95 \%$ and $92 \%$ lower for MOR and HOR, respectively. In the case of mortars with the highest BF content, this difference amounts to $84 \%$ and $86 \%$ for M1.5R and H1.5R, respectively. After 14 days of research, the differences between the hydrophobized and standard cement mortars were significantly lower and equalled $21 \%$ for M0R and $18 \%$ for M1.5R.

The obtained results indicate that the type of mortar governs the values of CA. The CA measurements showed that the contact angles of the hydrophobized cement mortars are significantly higher than in standard mortars (by up to $57 \%$ ). Conversely, the lowest contact angle value was obtained in the case of $\mathrm{H} 1.5$ mortar with $1.5 \% \mathrm{BF}$ content and reactive siloxanes addition. The contact angle drops as the fibres content increases, which is connected with higher porosity and absorptivity of mortars.

The greatest contact angle was observed for M0 mortars, both prior to and following hydrophobisation. In this case, the polysiloxane film changed the adhesion parameters by $57 \%$. Hydrophobisation reduced CA by $9.6 \%$ and $23 \%$ for $\mathrm{H} 0$ and $\mathrm{H} 1.5$, respectively. As the content of BF increased, CA exhibited a drop from $7 \%$ to $24.5 \%$ in Series 1 and from $13.7 \%$ to $35 \%$ in Series 2.

SFE in the case of all cement mortars before surface hydrophobisation is relatively high and indicates good hydrophilicity of mortars $\left(\mathrm{CA}<90^{\circ}\right.$ ). Hydrophobisation with polysiloxanes greatly reduced adhesion to mortar surface, which is connected with higher resistance to corrosive liquid infiltration, e.g., salty solutions. Even in the case of H1.5 mortars characterized by highest absorptivity, hydrophobisation turned out to be successful and reduced the negative impact of basalt fibres on wettability, because SFE of this mortar following hydrophobisation $\left(37 \mathrm{~mJ} \cdot \mathrm{m}^{-2}\right)$ is still $29.5 \%$ lower than the one of $\mathrm{M} 0$ reference mortars without basalt fibres.

The characteristics of surface properties, especially SFE, are considered important parameters required for understanding the mechanisms of surface phenomena $[12,22,43]$. SFE is the result of attraction between the molecules in the surface layer and volume of a material [43]. The literature includes papers on applying thin silane films on various substrates in order to modify their final wettability $[12,22,29,53]$. On the basis of the literature data on SFE of hydrophobized materials, it can be stated that there are few such studies, which was also observed by Cappalletti G. et al. [53]. The authors of [53] examined nine different types of hydrophobic films based on silanes, siloxanes, chlorosilanes, and others. In the majority of cases, the obtained SFE values did not exceed $42 \mathrm{~mJ} \cdot \mathrm{m}^{-2}$; for octadecyltrichlorosilane were lower than $23.5 \mathrm{~mJ} \cdot \mathrm{m}^{-2}$. CA over $120^{\circ}$ can be obtained through chemical modification of surface with siloxanes. The studies on SFE of silane and polisiloxane films were described, i.a. in the papers by Król P. et al. [54], Courard L. et al. [55], and Selvakumar N. et al. [56]. The paper [54] reports that SFE of silanes did not exceed $23 \mathrm{~mJ} \cdot \mathrm{m}^{-2}$, and approximately $42 \mathrm{~mJ} \cdot \mathrm{m}^{-2}$ in the case of triethoxysilanes. Polymer films reached SFE in the range of 21 to $45 \mathrm{~mJ} \cdot \mathrm{m}^{-2}$. In our work, similar and slightly lower SFE values were obtained for polysiloxane films in aqueous solvent, ranging from 10.53 to $37 \mathrm{~mJ} \cdot \mathrm{m}^{-2}$ depending on the content of $\mathrm{BF}$ and 
hydrophobising admixture. This indicates a very good hydrophobisation efficiency, especially in terms of protection against water and frost corrosion, which was described in Section 4.2.3.

It can be observed that roughness as well as SFE, and consequently wettability of the mortar surface increase along with the content of basalt fibres and siloxane admixture. The polysiloxane film reduces these parameters. Linear dependencies were observed between roughness and SFE; a regression model with a single variable was employed (Figure 10).

These correlations mainly stem from changes in the porosity of cement mortars depending on their composition and the applied preparation or hydrophobising admixture. This is because porosity is strictly connected with absorptivity, contact angle and SFE. Porosity is also connected with the compression strength. Figure 10 indicates that porosity drops as a result of hydrophobisation, however SFE mainly depends on the chemical changes occurring on the surface of material in the course of polysiloxane film application, not only on roughness. Despite similar roughness of the cement mortars, SFE is much lower for the surfaces covered with polysiloxane film. Siloxanes are capable of forming durable spatial cross-links. Owing to their chemical similarity, the hydrophobic substance forms lasting and resistant bonds with silanol groups present in hydrophilic construction materials after the contact with the mineral surface [43]. During the application of silicones on the surface of material, they bind their inorganic part $\mathrm{Si}-\mathrm{O}-\mathrm{Si}$ with the mineral substrate, directing non-wettable organic groups $\mathrm{R}$ outwards, resulting in a significant reduction of SFE value.

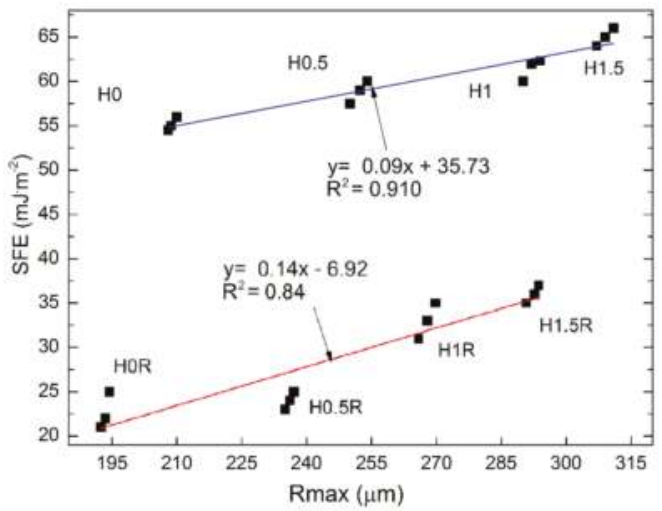

Figure 10. Correlation between roughness and surface free energy (SFE) of mortars with basalt fibres Series 2: blue line-unmodified; red line-hydrophobized surface.

\subsubsection{Frost resistance}

The frost resistance test did not exhibit significant differences in the behaviour of the cement mortars subjected to the F-T cycles. The hydrophobized samples (Figure $8 \mathrm{~b}, \mathrm{c}$ ) showed virtually no damage, similarly to the samples of M1 mortar without hydrophobisation, which were characterized by small chippings on the surface (Figure 8a). In the case of the mortars with reactive siloxane admixture, more serious damage of samples occurred, as presented in Figure 9. Loosening, flaking and losses were observed in the samples.

The greatest mass loss corresponds to H1.5 mortar and amounts to $8.3 \%$, which is over 10 times greater value than the mortar with the same BF content, but without siloxanes (M1.5). Siloxane admixture dramatically lowered the frost resistance of the mortar. The greatest reduction in strength after $50 \mathrm{~F}-\mathrm{T}$ cycles was observed for this cement mortar, equalling as high as $24.3 \%$, whereas the mortar without siloxanes experienced only a $2.5 \%$ reduction. Such situation has occurred because the hardening process of the polysiloxane gel was disrupted in the aqueous environment of cement slurry and hydration of cement in the presence of a polymer compound, which was mentioned in the 
Section 4.1 and SEM analyses. The admixture had a negative impact on the strength and frost resistance of the cement mortars with BF. However, the application of polysiloxanes as a protective film yielded the expected results. The mass loss of the cement mortars with $1.5 \%$ BF dropped almost three-fold, whereas the compression strength reduction decreased almost by the factor of five. Jiang et al. [10] showed that the cement mortars with basalt fibres ale characterised by lower early workability, better flexural strength, abrasion resistance, water permeability, but also a greater mass loss resulting from freezing and thawing, due to higher porosity, which was also indicated in our studies.

One of the principal parameters having an influence on the hydrophobisation efficiency is the adhesion of siloxane films to the substrate. During freezing and thawing, resulting from the infiltration of water through a polysiloxane gel film, delamination may occur at the contact spot between the material and film. No such situation occurred in the analyzed cement mortars with BF. All of the analyzed mortars have successfully protected the samples against the effect of frost. However, there are papers, including the authors' own works, which indicate that molecules of polysiloxane gel covered concrete too tightly, resulting in excessive ice crystallization pressure, which severely damaged the lightweight aggregate and the contact spot between the aggregate and cement paste [43]. Similar observations related to the high hydrophobisation efficiency were made in the work by Barnat-Hunek D. [43] concerning hydrophobized UHPC concretes with the addition of steel and polypropylene fibres. The concrete with steel fibres is especially vulnerable to frost corrosion. The application of methyl-silicone resins, particularly alkyl-alkoxy-siloxanes, has greatly increased the resistance of UHPC to F-T cycles. Even after 180 F-T cycles, in majority of cases the CA value was still greater than $100^{\circ}$, which indicates good hydrophobicity of concrete surface.

The corrosion resistance characterizing cement mortars is indirectly dependent on the frost resistance.

Frost and salt resistance are considered important elements for the assessment of concrete durability. The transition of water from the liquid to solid phase increases its volume by approximately $9 \%$. When the pores in concrete are almost completely saturated, freezing may cause serious cracks and damage of concrete.

Due to a strong correlation between roughness and SFE, the authors intended to prove that a correlation exists between the variable parameter connected with moisture, adhesion properties (SFE), and frost resistance. In this case, a drop in the strength of cement mortars following 50 F-T cycles corresponded to frost resistance. This parameter, expressed as a percentage of compression strength reduction, depends on SFE. This dependency can be described by means of a linear function (Figure 11).

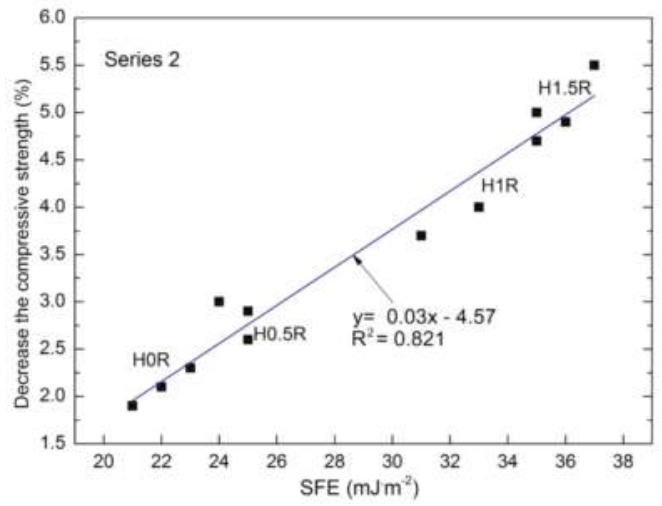

Figure 11. Correlation between SFE and frost resistance of mortars with basalt fibres. 
The correlations described by the formula $y=0.03 x-4.57$ indicate a good coefficient of determination $R^{2}=0.821$. Taking into account the data presented in Figure 11 and other obtained results, it can be concluded that both the moisture parameters, as well as adhesion properties (SFE) are closely connected with frost resistance. The lower the wettability and SFE, the higher the frost resistance. Protection of the cement mortars with BF against moisture, provided through surface hydrophobisation, has greatly increased frost resistance.

\section{Conclusions}

This work analyzed the physical properties as well as the microstructure characterizing the cement mortars with basalt fibres subjected to hydrophobisation.

On the basis of the analysis of research, the following conclusions were drawn:

1. The addition of BF has a negative impact on compression strength and frost resistance. However, it improves the flexural strength of the analyzed mortars with and without siloxane addition by $24 \%$ and $32 \%$, respectively.

2. The BF addition caused changes in the structure of cement mortars and altered the roughness parameters. Along with the increase of basalt fibres, porosity increases by up to $32 \%$.

3. Reactive polysiloxane applied in the Series 2 mixtures increased the average roughness by up to $31 \%$ for the standard mortars and by up $13 \%$ with the highest share of basalt fibres (H1.5). The polymer admixture causes creation of chemical bonds between particular concrete components, influencing the changes in the microstructure and the strength parameters. The hardening process of polysiloxane gel in the aqueous environment of cement slurry and cement hydration in the presence of polymer compound are disrupted. The presence of water weakens the resin hardening process, and the final polymer cross-linking reaction yielding a film of polysiloxane gel is disrupted.

4. A linear reaction between the roughness and adhesion properties described by SFE was presented. The highest hydrophobicity was obtained in the case of the reference MOR mortar with SFE equal to $10.53 \mathrm{~mJ} \cdot \mathrm{m}^{-2}$.

5. The investigation pertaining to the roughness following hydrophobisation, as well as the produced 3D profilograms, confirmed the influence of polysiloxane film on roughness reduction. The cement mortars without the polysiloxane film are characterized by four times greater adhesion, which is connected with the durability of mortars. Following hydrophobisation, the frost resistance of the mortars with $1.5 \%$ basalt fibres addition dropped almost three-fold, whereas the reduction in the compression strength decreased almost by a factor of five.

6. Due to the negative impact of siloxane admixture in the cement mortars with basalt fibres additionon the frost resistance, compression strength, and adhesion of cement paste to fibres, its application in this type of mortars is not recommended.

7. Due to a reduction in wettability and increase in frost resistance, performing the surface hydrophobisation by means of polysiloxanes is encouraged in order to improve the durability of cement mortars with basalt fibres.

Acknowledgments: This work was financially supported by the Ministry of Science and Higher Education in Poland within the statutory research number S/14/2018 and S-70/WIŚ/2018 and 2017-WNET/KNT/ZB/1.

Author Contributions: Danuta Barnat-Hunek conceived the idea of the experiment. Stanisław Fic and Danuta Barnat-Hunek conducted the experimental part. Monika Jarosz-Hadam, Danuta Barnat-Hunek, and Grzegorz Łagód analyzed the results and discussed the experimental results. Grzegorz Łagód translated the article. Danuta Barnat-Hunek, Grzegorz Łagód, and Monika Jarosz-Hadam provided manuscript formatting. Danuta Barnat-Hunek and Grzegorz Łagód provided substantive comments.

Conflicts of Interest: The authors declare no conflict of interest. 


\section{References}

1. Fiore, V.; Scalici, T.; Bella, G.D.; Valenza, A. A review on basalt fiber and its composites. Compos. B Eng. 2015, 74, 74-94. [CrossRef]

2. Morova, N. Investigation of usability of basalt fibres in hot mix asphalt concrete. Constr. Build. Mater. 2013, 47, 175-180. [CrossRef]

3. Militky, J.; Kovačič, V.; Rubnerová, J. Influence of thermal treatment on tensile failure of basalt. Eng. Fract. Mech. 2002, 69, 1025-1033. [CrossRef]

4. Ralegaonkar, R.; Gavali, H.; Aswath, P.; Abolmaali, S. Application of chopped basalt fibers in reinforced mortar, A review. Constr. Build. Mater. 2018, 164, 589-602. [CrossRef]

5. Dhand, V.; Mittal GRhee, K.Y.; Park, S.J.; Hui, D. A short review on basalt fiber reinforced polymer composites. Compos. B Eng. 2015, 73, 166-180. [CrossRef]

6. Kogan, F.M.; Nikitina, O.V. Solubility of chrysotile asbestos and basalt fibers in relation to their fibrogenic and carcinogenic action. Environ. Health. Persp. 1994, 102, 205-206. [CrossRef]

7. Hao, L.C.; Yu, W.D. Evaluation of thermal protective performance of basalt fiber nonwoven fabrics. J. Therm. Anal. Calorim. 2010, 100, 551-555. [CrossRef]

8. Moiseev, E.A.; Gutnikov, S.I.; Malakho, A.P.; Lazoryak, B.I. Effect of iron oxides on the fabrication and properties of continuous glass fibers. Inorg. Mater. 2008, 44, 1026-1030. [CrossRef]

9. Nasir, V.; Karimipour, H.; TBehrooz, F.; MShokrieh, M. Corrosion behavior and crack formation mechanism of basalt fiber in sulphuric acid. Corros. Sci. 2012, 64,1-7. [CrossRef]

10. Jiang, C.; Huang, S.; Zhu, Y.; Lin, Y.; Chen, D. Effect of polypropylene and basalt fiber on the behavior of mortars for repair applications. Adv. Mater. Sci. Eng. 2016, 2016, 1-11. [CrossRef]

11. Santarelli, M.L.; Sbardella, F.; Zuena, M.; Tirillo, J.; Sarasini, F. Basalt fiber reinforced natural hydraulic lime mortars: A potential bio-based material for restoration. Mater. Des. 2014, 63, 398-406. [CrossRef]

12. Barnat-Hunek, D.; Smarzewski, P. Influence of hydrophobisation on surface free energy of hybrid fiber reinforced ultra-high performance concrete. Constr. Build. Mater. 2016, 102, 367-377. [CrossRef]

13. Sadowski, Ł.; Stefaniuk, D.; Hoła, J. The effect of the porosity within the interfacial zone between layers on pull-off adhesion. Constr. Build. Mater. 2017, 152, 887-897. [CrossRef]

14. Sadowski, Ł.; Mathia, T.G. Review. Multi-scale metrology of concrete surface morphology: Fundamentals and specificity. Constr. Build. Mater. 2016, 113, 613-621. [CrossRef]

15. Halicka, A. Influence new-to-old concrete interface qualities on the behaviour of support zones of composite concrete beams. Constr. Build. Mater. 2011, 25, 4072-4078. [CrossRef]

16. Czarnecki, L.; Łukowski, P.; Garbacz, A. The Protection and Repair of Concrete Structures. In Comment to PN-EN 1504; PWN: Warsaw, Poland, 2016. (In Polish)

17. Sadowski, Ł.; Hoła, J.; Czarnecki, S. Non-destructive neural identification of the bond between concrete layers in existing elements. Constr. Build. Mater. 2016, 127, 49-58. [CrossRef]

18. Wu, S. Polymer Interface and Adhesion; CRC Press: Boca Raton, FL, USA, 1982.

19. Quere, D. Rough ideas on wetting. Physica A 2002, 313, 32-46. [CrossRef]

20. Wenzel, R.N. Resistance of solid surfaces to wetting by water. Ind. Eng. Chem. 1936, 28, 988-994. [CrossRef]

21. Bico, J.; Tordeux, C.; Quéré, D. Rough wetting. Europhys. Lett. 2001, 55, 214-220. [CrossRef]

22. Barnat-Hunek, D.; Łagód, G.; Siddique, R. Properties of hydrophobised lightweight mortars with expanded cork. Constr. Build. Mater. 2017, 155, 15-25. [CrossRef]

23. Xue, X.; Li, Y.; Yang, Z.; He, Z.; Dai, J.-G.; Xu, L.; Zhang, W. A systematic investigation of the waterproofing performance and chloride resistance of a self-developed waterborne silane-based hydrophobic agent for mortar and concrete. Constr. Build. Mater. 2017, 155, 939-946. [CrossRef]

24. Czarnecki, L.; Garbacz, A.; Krystosiak, M. On the ultrasonic assessment of adhesion between polymer coating and concrete substrate. Cem. Concr. Compos. 2006, 28, 360-369. [CrossRef]

25. Suchorab, Z.; Barnat-Hunek, D.; Franus, M.; Łagod, G. Mechanical and physical properties of hydrophobized lightweight aggregate concrete with sewage sludge. Materials (Basel) 2016, 9, 317. [CrossRef] [PubMed]

26. Brzyski, P.; Barnat-Hunek, D.; Fic, S.; Szelag, M. Hydrophobisation of lime composites with lignocellulosic raw materials from flax. J. Nat. Fibers. 2017, 14, 609-620. [CrossRef]

27. Tittarelli, F. Oxygen diffusion through hydrophobic cement-based materials. Cem. Concr. Res. 2009, 39, 924-928. [CrossRef] 
28. Fic, S.; Szewczak, A.; Barnat-Hunek, D.; Łagód, G. Processes of Fatigue Destruction in NanopolymerHydrophobised Ceramic Bricks. Materials 2017, 10, 44. [CrossRef] [PubMed]

29. Barnat-Hunek, D.M.; Widomski, K.; Szafraniec, M.; Łagód., G. Impact of Different Binders on the Roughness, Adhesion Strength, and Other Properties of Mortars with Expanded. Cork. Mater. 2018, 11, 364. [CrossRef] [PubMed]

30. Jian-jun, L.; Ying, M.; Yan-chun, L. The performance of green basalt fiber and its application in the civil engineering field. Appl. Mech. Mater. 2012, 193-194, 548-552.

31. Kamiya, S.; Tanaka, I.; Imamura, K.; Sasaki, H.; Nakagawa, N. Basalt fiber material. US Patent 7,767,603 B2, 3 August 2010.

32. Barnat-Hunek, D.; Smarzewski, P. Analysis of the physical properties of hydrophobised lightweightaggregate mortars. Compos. Theory Pract. 2016, 16, 96-102.

33. PN EN 197-1:2012 Cement-Part 1: Composition, Specifications and Conformity Criteria for Common Cements; PKN: Warsaw, Poland, 2012.

34. EN 196-1:2016-07 Methods of Testing Cement_Part 1: Determination of Strength; BSI: London, UK, 2016.

35. PN-EN 1015-10:2001 Methods of Test Mortar for Masonry - Vol. 10: Determination of Density of the Dry Hardened Mortar; PKN: Warsaw, Poland, 2001.

36. PN-EN 1015-11:2001 Methods of Testing Mortars for Walls-Part 11: Determination of Flexural Strength and Compressive Strength of the Hardened Mortar; PKN: Warsaw, Poland, 2001.

37. BS 1881-122:2011 Testing Concrete. Method for Determination of Water Absorption; BSI: London, UK, 2011.

38. PN-EN ISO 7783:2012 Farby i Lakiery-Oznaczanie WłAśCiwośCi Przenikania Pary Wodnej. Metoda z Zastosowaniem Naczynka; PKN: Warsaw, Poland, 2012.

39. PN-B-12012:2007 Methods of Test for Masonry Units—Determination of Resistance to Freeze-Thaw Masonry Ceramic; PKN: Warsaw, Poland, 2007.

40. Santos, P.M.D.; Júlio, E.N.B.S. A state-of-the-art review on roughness quantification methods for concrete surfaces. Constr. Build. Mater. 2013, 38, 912-923. [CrossRef]

41. Neumann, A.W.; Good, R.J.; Hope, C.J.; Sejpal, M. An equation-of-state approach to determine surface tensions of low-energy solids from contact angles. J. Colloid Interface Sci. 1974, 49, 291-304. [CrossRef]

42. Łukowski, P. Role of polymers in forming of properties of polymer-cement binders and composites [in Polish], Prace Naukowe Politechniki Warszawskiej. Budownictwo 2008, 148, 3-159.

43. Czarnecki, L.; Łukowski, P. Importance of Interphase Surfaces in Concrete-Like Polimer Composites Composites [In Polish]; I Konferencja Naukowa: Inżynieria Materiałowa, Bydgoszcz, 1989; pp. 97-114.

44. Shaker, F.A.A.; El-Dieb, S.; Reda, M.M. Durability of Styrene-Butadiene latex modified concrete. Cem. Concr. Res. 1997, 27, 711-720. [CrossRef]

45. Palou, M.T.; Bagel, L.; Zivica, V.; Kuliffayova, M.; Kozankova, J. Influence of hydrothermal curing regimes on the hydration of fiber-reinforced cement composites. J. Therm. Anal. Calorim. 2013, 113, 219-229. [CrossRef]

46. Quattrociocchi, G.; Albe, M.; Tirillo, J.; Sarasini, F.; Valente, M.; Santarelli, M.L. Basalt fibers as a sustainable reinforcement for cement based mortars: Preliminary study, materials characterization VII 109. WIT Trans. Eng. Sci. 2015, 90, 109-120. [CrossRef]

47. Ma, J.; Zhang, M.; Zhao, G. Experimental research on basalt fiber reinforced cementitious composites. Appl. Mech. Mater. 2013, 253-255, 533-536. [CrossRef]

48. Dong, Q.Q.; Jiang, C.H.; McCarthy, T.J.; Chen, D. Influence of basalt fiber on performance of cement mortar. Key Eng. Mater. 2010, 426-427, 93-96. [CrossRef]

49. Semenov, V.; Rozovskaya, T.; Gubsckiy, A.; Gareeva, R. Effective light-weight masonry mortars with dispersed reinforcement. Proc. Eng. 2016, 153, 630-637. [CrossRef]

50. Li, Z.; Bu, N.; Cong, X. Contrast experiment of common crack repair method and comprehensive new repair technology research. Appl. Mech. Mater. 2012, 166-169, 1587-1591. [CrossRef]

51. Hay, K.M.; Dragila, M.I.; Liburdy, J. Theoretical model for the wetting of a rough surface. J. Colloid Interface Sci. 2008, 325, 472-477. [CrossRef] [PubMed]

52. Barnat-Hunek, D. SURFACE Free Energy as a Factor Affecting Hydrophobisation Effectiveness in Protection of Building Construction; Publishing House of Lublin university of Technology: Lublin, Poland, 2016; 230p, ISBN 978-83-7947-216-1. 
53. Cappelletti, G.; Ardizzone, S.; Meroni, D.; Soliveri, G.; Ceotto, M.; Biaggi, C.; Benaglia, M.; Raimondi, L. Wettability of bare and fluorinated silanes: A combined approach based on surface free energy evaluations and dipole moment calculations. J. Colloid Interface Sci. 2013, 389, 284-291. [CrossRef] [PubMed]

54. Król, P.; Lechowicz, J.B.; Król, B. Modelling the surface free energy parameters of polyurethane coats-Part 1. Solvent-based coats obtained from linear polyure-thane elastomers. Colloid Polym. Sci. 2013, 291, 1031-1047. [CrossRef] [PubMed]

55. Courard, L.; Michel, F.; Perkowicz, S.; Garbacz, A. Effects of limestone fillers on surface free energy and electrical conductivity of the interstitial solution of cement mixes. Cem. Concr. Comp. 2014, 45, 111-116. [CrossRef]

56. Selvakumar, N.; Barshilia, H.C.; Rajam, K.S. Effect of substrate roughness on the apparent surface free energy of sputter deposited superhydrophobicpolytetra-fluoroethylene coatings: A comparison of experimental data with different theore-tical models. J. Appl. Phys. 2010, 108, 013505. [CrossRef]

(C) 2018 by the authors. Licensee MDPI, Basel, Switzerland. This article is an open access article distributed under the terms and conditions of the Creative Commons Attribution (CC BY) license (http:/ / creativecommons.org/licenses/by/4.0/). 
Article

\title{
Non-Leachable Hydrophilic Additives for Amphiphilic Coatings
}

\author{
Guillaume Gillet *, Fabrice Azemar, Fabienne Faÿ, Karine Réhel and Isabelle Linossier \\ University of Southern Brittany, EA 3884, Laboratoire de Biotechnologie et Chimie Marines (LBCM), \\ Institut Universitaire Européen de la Mer IUEM, F-56100 Lorient, France; fabrice.azemar@univ-ubs.fr (F.A.); \\ fabienne.fay@univ-ubs.fr (F.F.); karine.rehel@univ-ubs.fr (K.R.); isabelle.linossier@univ-ubs.fr (I.L.) \\ * Correspondence: guillaume.gillet@univ-ubs.fr; Tel.: +33-627001239
}

Received: 23 March 2018; Accepted: 12 April 2018; Published: 16 April 2018

\begin{abstract}
Amphiphilic surfaces are particularly effective at inhibiting the adhesion of microorganisms (bacteria, cells, microalgae, etc.) in liquid media. The aim of this study is to determine the best hydrophilic linker to promote bonding between poly(ethylene glycol) (PEG) as a hydrophilic additive and poly(dimethyl siloxane) (PDMS) as the hydrophobic matrix. Various parameters have been studied (molecular weight, linker type, and polymer end-group), as well as the efficiency of the linking, the capacity of PEG to access to the surface of the film, and overall film homogeneity. According to the results, a PDMS linker paired with a PEG moiety allows for compatibilization of the compounds during cross-linking. This compatibilization seems to provide a good bonding with the matrix and a good surface access to the hydrophilic moiety. Therefore, this structure comprising a linking function attached to the PDMS-PEG copolymer has high potential as a non-releasable additive for amphiphilic coating applications.
\end{abstract}

Keywords: poly(ethylene glycol) (PEG); hydrophilic; non-releasable; polydimethylsiloxane; coatings; cross-linking; surface; amphiphilic; anti-bioadhesion

\section{Introduction}

Silicone elastomers such as poly(dimethyl siloxane) (PDMS) have received great attention regarding their potential use as anti-bioadhesion coatings [1]. PDMS is a soft polymer with attractive chemical and physical properties such as biocompatibility, low toxicity, flexible surface chemistry, low permeability to water, elastomeric properties, ease of fabrication and use, as well as low manufacturing costs [2]. It is widely used to reduce or prevent bioadhesion in biomedical field [3-8]. However, these kinds of coatings are prone to protein adsorption and accumulation of strongly adherent diatom slimes [9]. The addition of oil (e.g., amphiphilic copolymers) is a common technique for enhancing the biofouling-resistance properties of silicone-matrix fouling-release coatings [10]. Kavanagh et al. [11] have used two PDMS-based fluids that significantly reduced the adhesion strength of barnacles compared to unmodified elastomers. It has been reported that these "oils" are usually block copolymers that can migrate through the coatings to function as surfactants and produce a weak boundary layer affecting the surface properties [12,13]. Poly(ethylene glycol) (PEG) has been widely used as an additive for fouling release [14-16]. Camos Noger et al. [17] studied the diffusion ability of PEG-based amphiphilic compounds containing different hydrophobic components through a PDMS matrix via contact angle measurements. Wu et al. modified a PDMS surface with a PEG-based block copolymer to improve the anti-protein effect with respect to fluorescent tagged antibody adhesion [18]. The lubricity of PDMS surfaces has also been increased by using poly(acrylic acid)- and PEG-based block copolymers, as shown by Røn et al. [19]. Sundaram et al. [9] used the combination of PDMS and PEG chains to test the advantages over conventional silicones against diatom fouling [17,20]. Silicone oils have been classified as hazardous substances and cannot be released into water environments. 
While no hazards are substantiated for silicone resins, PDMS fluids are a matter of concern. Their inert oily nature and relative extreme persistence are a potential threat to aqueous environments as well as the human body. According to Nendza et al. [21], a favorable option may be the use of silicone-based coatings formulated without leaching silicone oils. This option may be achieved by using compounds with an ability to chemically bind to the network during cross-linking [22].

The aim of this study is to determine the optimal method of incorporating sustainable hydrophilic compounds into silicone coatings to obtain one with amphiphilic surface properties. The ability of the hydrophilic compound to remain in the coating and to access the surface presents two advantages: the durability of amphiphilic properties is increased, and the release of polymers into the environment or the body is avoided.

In this study, a hydrophilic PEG-based component was introduced into a PDMS matrix. The PDMS used contains methoxysilane end groups, which, in the presence of water and catalyst, can cross-link and form a tridimensional network. In this network, different sizes of PEG have been incorporated in an un-cross-linked blend that (1) is able to react with the cross-linking group (methoxysilane) of the PDMS network, (2) contains "unreactive" groups with respect to methoxysilane as a negative reference, and (3) contains a linker (PDMS, alkyl chain $\mathrm{C}_{14}$, or alkyl chain $\mathrm{C}_{8}$ ) to be used as a compatibilizer. The un-cross-linked blend was applied to the surface of polycarbonate and glass to proceed with different tests after curing. The cross-linking potential was then evaluated via soxhlet extraction and a swelling test. The surface accessibility potential of the hydrophilic compounds was characterized by contact angle measurements. Lastly, the surface homogeneity was observed by scanning electron microscopy.

\section{Materials and Methods}

\subsection{Materials}

Poly(ethylene glycol) methyl ether ( $\left.M_{\mathrm{n}}: 2000 \mathrm{~g} / \mathrm{mol} \mathbf{P E G} 45\right)$, pentenoic acid 97\%, $N, N^{\prime}$-dicyclohexylcarbodiimide $99 \%$, 4-dimethylaminopyridine $>99 \%$, tetraethyl orthosilicate reagent grade $98 \%$, azobisisobutyronitrile $98 \%$, and phosphoric acid $85 \%$ in water were acquired from Sigma-Aldrich (Saint-Louis, MO, USA). Hydroxyl-terminated poly(ethylene glycol) $\left(M_{\mathrm{n}}: 400 \mathrm{~g} / \mathrm{mol}\right.$, PEGg) was provided by Biochemica (Billingham, UK). Tegomer ${ }^{\circledR}$ (Essen, Germany) H-Si 2315 polydimethylsiloxane bis(hydroxyl) $\left(M_{\mathrm{n}}: 2315 \mathrm{~g} / \mathrm{mol}\right.$ per manufacturer's specifications, $\left.\mathbf{P D M S} \mathbf{2}_{28}\right)$ was kindly supplied by EVONIK (Essen, Germany). (3-Mercaptopropyl)trimethoxysilane $>96 \%$ was purchased from TCI (Tokyo, Japan). PDMS-SiOH (copolymer PEG-PDMS, mono Si-OH end function, which may react with cross-linking function, pendant chain PEG per manufacturer specifications) and 3-(triethoxysilyl)propyl isocyanate $98 \%$ are commercially available (Momentive, Waterford, NY, USA) and were used in bulk reaction ( $1 / 1.05$ equiv, $\mathrm{N}_{2}, 20^{\circ} \mathrm{C}$ ) to form PDMS-TMS, which was ended with bis(trimethoxysilane) and could react with cross-linking functions Trimethoxysilane-terminated poly(ethylene glycol) $\left(M_{\mathrm{n}}: 350 \mathrm{~g} / \mathrm{mol} \mathbf{P E G}_{6}\right)$ was also purchased from Momentive. US-CF 2403 methyl siloxane resin with methoxy function was provided by Dow Corning (Midland, MI, USA) $\left(M_{\mathrm{W}}<1000 \mathrm{~g} / \mathrm{mol}\right.$ per manufacturer specifications) and was named PDMS $_{13}$. All products were used as received.

\subsection{Synthesis and Coating}

\subsubsection{Synthesis of Bis(Trimethoxysilane)-Terminated Poly(Dimethyl Siloxane) ( $\left.\mathrm{PDMS}_{28}\right)$}

PDMS $_{28}$ was prepared via Steglich esterification followed by a thiol-ene reaction. Firstly, dihydroxyalkyl poly(dimethyl siloxane) (15 g, 1 equiv), pentenoic acid (1.94 g, 3 equiv), $N, N^{\prime}$-dicyclohexylcarbodiimide ( $8.08 \mathrm{~g}, 6$ equiv), and 4-dimethylaminopyridine $(0.397 \mathrm{~g}, 0.5$ equiv) were dissolved in $30 \mathrm{~mL}$ of tetrahydrofuran (THF). The mixture was degassed by a nitrogen flow, and the reaction occurred over $48 \mathrm{~h}$ under reflux at $75^{\circ} \mathrm{C}$. After quenching the reaction, the medium was filtered 
and solvent was removed. The products were solubilized in $40 \mathrm{~mL}$ of hexane and washed 5 times with $5 \mathrm{~mL}$ of 50:50 acetonitrile-methanol. The hexane phase was evaporated under vacuum, and the product was characterized via ${ }^{1} \mathrm{H}$ NMR in deuterated chloroform. Thereafter, the yellow oily product was mixed with azobisisobutyronitrile $(0.1968 \mathrm{~g}, 0.2$ equiv) and (3-mercaptopropyl)trimethoxysilane $(2.59 \mathrm{~g}, 2.2$ equiv) and dissolved in THF $(30 \mathrm{~mL})$. After degassing with nitrogen, thiol-ene reaction occurred over $24 \mathrm{~h}$ under reflux at $80^{\circ} \mathrm{C}$. The medium was carefully removed to avoid any contact with ambient air. A small part of the product was then collected, purified with process previously reported for the Steglich esterification, and analyzed by ${ }^{1} \mathrm{H}$ NMR.

\subsubsection{Functionalization of $\mathrm{PEG}_{45}$ with Short Alkyl Chain $\left(\mathrm{C}_{8}\right)$ and Trimethoxysilane End Groups (45-Alk8-TMS)}

45-Alk8-TMS was prepared using Steglich esterification and thiol-ene (Figure 1) of PEG $_{45}$ ( $1 \mathrm{~g}, 1$ equiv), pentenoic acid ( $0.1 \mathrm{~g}, 2$ equiv), $N, N^{\prime}$-dicyclohexylcarbodiimide ( $0.206 \mathrm{~g}, 2$ equiv), and 4-dimethylaminopyridine $(0.0305 \mathrm{~g}, 0.5$ equiv) were dissolved in dichloromethane $(5 \mathrm{~mL})$. The mixture was degassed by a nitrogen flow and the reaction occurred for $48 \mathrm{~h}$ under reflux at $75^{\circ} \mathrm{C}$. After quenching the reaction, the medium was filtered, and the solvent was removed. Products were solubilized in low THF volume and precipitated in diethyl ether. The solid was gathered and dried under vacuum. The product was characterized by ${ }^{1} \mathrm{H}$ NMR and thereafter ( $2 \mathrm{~g}, 1$ equiv) was mixed with azobisisobutyronitrile ( $0.0656 \mathrm{~g}$, 0.2 equiv), (3-mercaptopropyl)trimethoxysilane $(0.216 \mathrm{~g}, 1.1$ equiv) and finally dissolved in THF $(5 \mathrm{~mL})$. The medium was placed under nitrogen, and the thiol-ene reaction occurred over $24 \mathrm{~h}$ under reflux at $80^{\circ} \mathrm{C}$. Afterward, while avoiding any contact with ambient air, the solvent was removed under vacuum. A small part of the product was then collected and purified before the Steglich esterification process reported above, and finally analyzed via ${ }^{1} \mathrm{H}$ NMR.

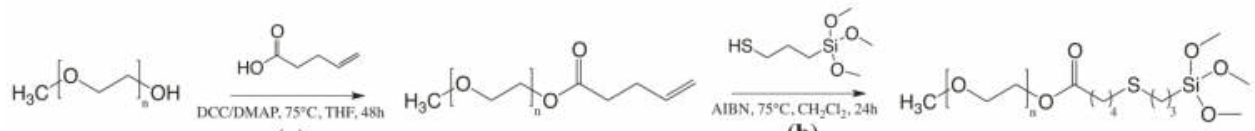

(a)

(b)

Figure 1. Synthesis of poly(ethylene glycol) (PEG) with short alkyl chain $\left(\mathrm{C}_{8}\right)$ and trimethoxysilane end group. (a) Steglich esterification. (b) Thiol-ene coupling.

\subsubsection{Functionalization of $\mathrm{PEG}_{45}$ with Alkyl Chain $\mathrm{C}_{14}$ and Trimethoxysilane End Groups (45-Alk14-TMS)}

45-Alk14-TMS was prepared using the same procedure described above for 45-Alk8-TMS The products used for Steglich esterification were poly(ethylene glycol) 45 ( $1 \mathrm{~g}, 1$ equiv), undecenoic acid ( $0.186 \mathrm{~g}, 2$ equiv), $N, N^{\prime}$-dicyclohexylcarbodiimide ( $0.206 \mathrm{~g}, 2$ equiv), and 4-dimethylaminopyridine ( $0.0305 \mathrm{~g}, 0.5$ equiv). The product obtained ( $2 \mathrm{~g}, 1$ equiv) was mixed with azobisisobutyronitrile ( $0.658 \mathrm{~g}, 0.2$ equiv) and (3-mercaptopropyl)trimethoxysilane ( $0.213 \mathrm{~g}, 1.1$ equiv) and, after treatment, was analyzed by ${ }^{1} \mathrm{H}$ NMR.

\subsubsection{Film Preparation}

The structure was composed of different number of ethylene oxide units (poly(ethylene glycol)) attached with a linker " $\mathrm{R}$ " to the polymer terminal function " $\mathrm{X}$ " (Figure 2). Details about the number of units, the structure of the linker, and the structure of the terminal function can be found in Table 1 . The reference sample was composed of PDMS (13 or 28 units), to study the influence of the PDMS' molecular weight, the ethanol (50 wt \% of the PDMS), and the phosphoric acid (10 wt \% of the PDMS). The PEG-based additives (10 wt \% of the PDMS) were first mixed with ethanol, the PDMS bis(trimethoxysilane) end-groups were then placed into a small vial, and the phosphoric acid was 
added (10 wt \% of the PDMS). After each addition, the reaction medium was homogenized for $2 \mathrm{~min}$ with a vortex. The product was coated on a polycarbonate (PC) sheet or a glass slide with a wet film thickness of $200 \mu \mathrm{m}$. Glass slides and PC sheets were cured at $20^{\circ} \mathrm{C}$ for $48 \mathrm{~h}$, and the samples were then stored in a drying oven at $30^{\circ} \mathrm{C}$. The film was removed from the PC sheets so that swelling test, soxhlet extraction, and scanning electron microscopy could proceed.

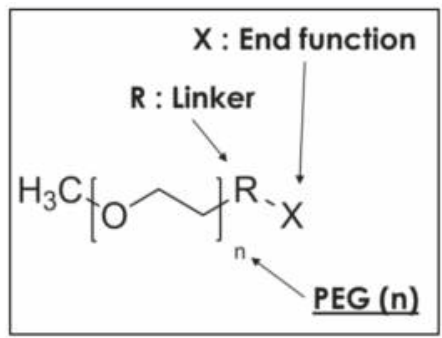

Figure 2. Structure of PEG-based additives.

Table 1. Composition of hydrophilic additives of poly(dimethyl siloxane) (PDMS) coatings.

\begin{tabular}{ccccc}
\hline n: PEG & R: Linker & X: End Function & Samples & States of Additive \\
\hline 0 & - & & Reference & - \\
6 & - & Trimethoxysilane & 6-TMS & Liquid \\
9 & - & Hydroxy & 9-OH & Liquid \\
X & PDMS & Silanol & PDMS-SiOH & Liquid \\
X & PDMS & Trimethoxysilane & PDMS-TMS & Liquid \\
45 & - & Hydroxy & 45-OH & White solid \\
45 & Alkyl C & Trimethoxysilane & 45-Alk8-TMS & Yellow solid \\
45 & Alkyl C 14 & Trimethoxysilane & 45-Alk14-TMS & Yellow solid \\
\hline
\end{tabular}

\subsection{Characterizations}

\subsubsection{Nuclear Magnetic Resonance}

${ }^{1} \mathrm{H}$ NMR spectra were obtained on a Spinsolve Benchtop NMR $60 \mathrm{MHz}$ and proceeded in $\mathrm{CDCl}_{3}$. The esterification yield of PDMS was determined by the shift of protons close to the reactive function, 3.34-3.98 ppm (m, $\left.2 \mathrm{H}, \mathrm{CH}_{2} \mathrm{OH}\right)$. Esterification of $\mathrm{PEG}$ was confirmed by the apparition of allyl protons peak, 6.34-5.36 ppm (m, 2H, $\left.\mathrm{CH}_{2} \mathrm{CHCH}_{2}\right)$ and 5.34-4.62 ppm (m, $\left.4 \mathrm{H}, \mathrm{CH}_{2} \mathrm{CHCH}_{2}\right)$, and protons peak close to the ester function, 3.96-4.43 ppm $\left(\mathrm{m}, 4 \mathrm{H}, \mathrm{CH}_{2} \mathrm{COOCH}_{2}\right)$. Thiol-ene coupling yield for all compounds was determined by the decrease of allyl protons peaks.

\subsubsection{Scanning Electron Microscopy}

Coatings were observed with an electron microscope JEOL 6460 LV equipped with an Oxford Inca 300 X-Ray microanalysis. Samples were separated from the polycarbonate sheet and placed on the metallization support. The surface was then metallized with carbon to obtain a conductive material. Each sample was scanned, and a screenshot of the most representative global architecture was taken (magnification $100 \times$ ). For all investigations, the beam energy was $20 \mathrm{kV}$.

\subsubsection{Soxhlet Extraction}

The amount of un-cross-linked material in a film cured on a polycarbonate was determined by soxhlet extraction. The film was extracted with chloroform in a soxhlet device for $24 \mathrm{~h}$. The weight loss was calculated as the weight difference between the extracted and unextracted weight divided by the unextracted weight. 


\subsubsection{Contact Angle Measurement}

Measurements were taken at room temperature with a contact angle system (Digidrop GBX, Dublin, Ireland) equipped with a syringe, a video camera, and an acquisition of angle measurements. The film was cured on the glass slide and then put in a drying oven at $30{ }^{\circ} \mathrm{C}$ for a week before contact measurements to have repeatable conditions. The contact angle of a $2 \mu \mathrm{L}$ drop of water was measured at 0,15 , and $30 \mathrm{~s}$ after contact between the drop and the film surface. The indicated values are an average of 5-8 measurements taken on different areas of three different films.

\section{Results}

\subsection{Synthesis}

\subsubsection{Synthesis of Dihydroxylalkyl Polydimethylsiloxane $\left(\mathrm{PDMS}_{28}\right)$}

Dry product was analyzed by ${ }^{1} \mathrm{H}$ NMR. The shift of protons from 3.34-3.98 to 3.76-4.40 ppm is due to the transformation of the hydroxyl group into an ester group (Figures S1). Its relative intensity compares to the one of the dimethyl peaks in the PDMS backbone to give a yield close to $100 \%$. Moreover, the presence of peaks corresponding to allyl protons at 6.34-5.36 ppm and 5.34-4.62 ppm and their relative intensities of $2 \mathrm{H}$ and $4 \mathrm{H}$, respectively, confirm the high yield obtained. Analysis of the ${ }^{1} \mathrm{H}$ NMR spectrum after the thiol-ene reaction shows a decrease of the allyl protons peaks. The yield calculated from the relative intensities is $93 \%$. Moreover, the presence of a methoxy proton peak (3.43-3.65 ppm) after purification confirms the end-group modification. (Figures S1 and S2).

\subsubsection{Poly(Ethylene Glycol) End-Group Modifications}

${ }^{1} \mathrm{H}$ NMR spectra of purified compounds obtained after the Steglich esterification show peaks corresponding to allyl protons. These peaks confirm the end-group modification for both linker types with different sizes (Figure S3 and S4).

As with the thiol-ene reaction of PDMS, the decrease of allyl proton peaks on spectra confirm the coupling between the PEG and the (3-mercaptopropyl)trimethoxysilane. Relative intensities indicate a yield of 74\% for 45-Alk8-TMS and 78\% for 45-Alk14-TMS.

\subsection{Film Preparation}

The solid additives 45-Alk14-TMS, 45-Alk8-TMS, and 45-PEG-OH were solubilized with the addition of ethanol at $50 \mathrm{wt} \%$ of PDMS matrix weight. These PEG-based additives contain 45 ethylene oxide (EO) units, making their melting points higher than room temperature, which explains their solid state. They have to first be solubilized before their incorporation in the coatings for good dispersion [23]. The amount of matrix, solvent, catalyst, and additives for each film were kept consistent. This allows for samples to be compared under similar cross-linking conditions, with the only differing factor as the additive. Coatings obtained after $48 \mathrm{~h}$ in the oven are colorless, smooth, and without cracks.

\subsection{Scanning Electron Microscopy}

The PDMS reference presented a smooth surface indicating that the cross-linking system and the catalyst-solvent combination-proportion are efficient. The coatings prepared with PDMS $_{\mathbf{2 8}}$ present the best results for film containing liquid hydrophilic additives as PDMS-TMS. Nevertheless, their surfaces are less homogeneous, the linking of hydrophilic additives is less important and there is no surface accessibility compared to those with $\mathbf{P D M S}_{13}$. In addition, the surface presents a high amount of bubbles and drying defaults due to its higher cross-linking speed. The crosslinking and solvent-catalyst proportion are not suitable for $\mathbf{P D M S}_{\mathbf{2 8}}$ (Figure 3).

Surface homogeneity is essential for avoiding bio-adhesion: Dantas et al. [24] observed that reduction in the surface roughness was directly related to a decrease in bacterial adhesion. To have amphiphilic character over the whole surface, it is important to have a homogeneous material. 
The surface topography of $\mathbf{P D M S}_{\mathbf{1 3}}$-based films is shown in Figure 3. There are two main types of surface films. The first is a smooth and homogeneous surface shown in Figure 3a,c for 6-TMS and PDMS-TMS, respectively. The PDMS reference, 9-OH, and PDMS-SiOH coatings exhibited similar surface properties. This smooth and homogeneous surface is characteristic of PDMS reference and four other coatings that contain only liquid hydrophilic additive with fewer EO repeat units. The additives 6-TMS and 9-OH contain 6 and 9 EO units, respectively. The PDMS-SiOH and PDMS-TMS contain 20-50 EO units per PDMS chain and should be solid according to Majumdar et al. [23]. However, the EO units are split into several low repeat PEG branches along the PDMS backbone, so the product remains in a liquid state even with an EO content superior to 13 units. These compounds are present in the liquid state and remain liquids after drying. Thus, film homogeneity should not be disrupted by samples with higher EO content adopting a solid state. Surface homogeneity seems to be impacted by the number of consecutive EO units in the PEG chain, as opposed to the total. The second typical profile, which presents with aggregation and a rough surface, is shown in Figure $3 b$, d for 45-OH and 45-Alk8-TMS, respectively. The 45-Alk14-TMS sample was found to have the same kind of surface, but it is not shown here. This profile is thought to be due to solidification of the PEG. Indeed, PEG at $20^{\circ} \mathrm{C}$ with $13 \mathrm{EO}$ units or fewer $\left(600 \mathrm{~g} \cdot \mathrm{mol}^{-1}\right)$ are in a liquid state, and those above 13 units are present in a solid state [23]. These products have $45 \mathrm{EO}$ units and are present in a solid state at $20^{\circ} \mathrm{C}$ as expected. The heterogeneous surface and phase segregation is believed to be caused by the return of PEG to its solid state during the drying process. It has been noted that phase compatibilization was not improved despite the use of $\mathrm{C}_{8}$ and $\mathrm{C}_{14}$ linkers. The number of consecutive units of $\mathrm{EO}$ chain seems to be the only impacting factor.

Short linear PEG additives (where EO units < 13) or PEG-PDMS copolymers (where EO units < 13) are a good solution for obtaining homogeneous surfaces [23]. We conclude that it is also possible to use a copolymer with a pendant chain that allows for the incorporation of several EO units while keeping it in a liquid state and preserving the homogeneity of the surface.
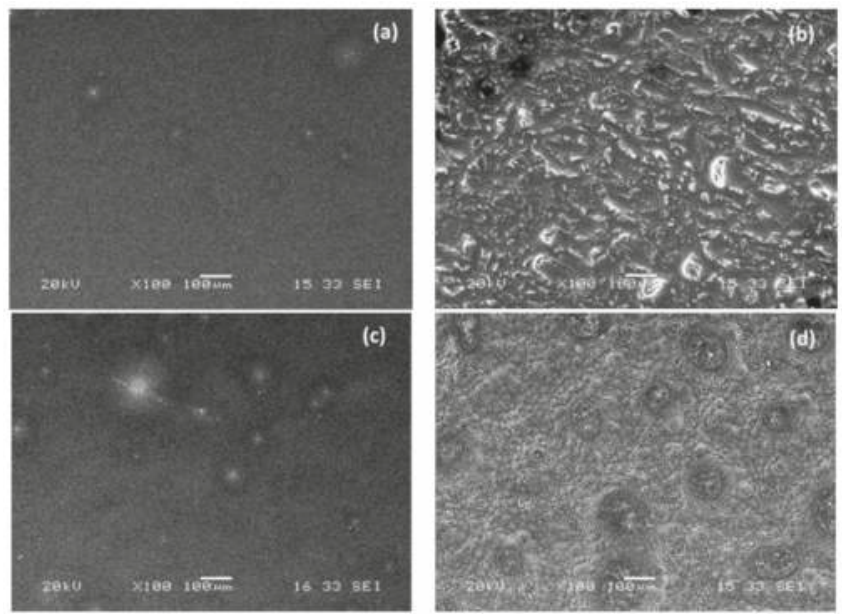

Figure 3. Scanning electron microscopy. Homogeneity of $\mathrm{PDMS}_{13}$-methoxy-ended cross-linked film with hydrophilic compounds incorporated (a) 6-TMS (b) 45-OH (c) PDMS-TMS, and (d) 45-Alk8-TMS. 


\subsection{Soxhlet Extraction}

Chemical additive retention was tested on hydrophilic compounds with varying cross-linking function and linkers. This included evaluating PEG of different molecular weights to study the entanglement possibilities between the two compounds.

Leaching potential of the different hydrophilic compounds inside the $\mathbf{P D M S} \mathbf{M S}_{\mathbf{1 3}}$ and $\mathbf{P D M S} \mathbf{S}_{\mathbf{2}}$ were tested. Figure 4 shows the weight loss of each coating. The PDMS reference had a higher weight loss $(4.7 \%)$ when compared to a similar study by Grunlan et al. [25]. This could be due to the catalyst amount and the length of PDMS chain in the silicone sample that was used. Higher catalyst proportions accelerate cross-linking, decrease the chain mobility, and lead to structure immobilization. Short PDMS chains decrease the network flexibility and therefore the chain mobility, leading to a faster structure immobilization (freezing). This freeze process stops the reaction and hinders chain linking to the matrix. These chains will be thereafter extracted by solvent and contribute to the weight loss.

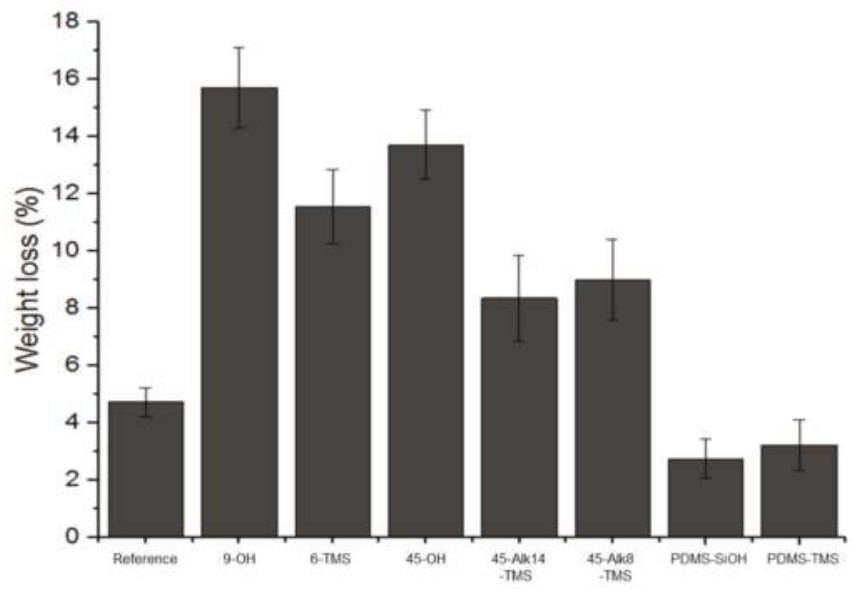

Figure 4. Uncross-linked materials and hydrophilic additives extracted from the film of PDMS $_{\mathbf{1 3}}$

In Figure 4, coatings containing 9-OH, 6-TMS, and 45-OH presented high amounts of weight loss $(15.7,11.6$, and $13.7 \%$, respectively) consistent with the absence of linking. Differences in these samples were not significant based on their standard deviations. As mentioned, weight loss from the hydrophilic additives $9-\mathrm{OH}$ and $45-\mathrm{OH}$ are due to a lack of linking. In addition, increasing the number of EO units to 45 does not provide any noticeable entanglement effects between the PEG and PDMS network. The 6-TMS structure is characterized by trimethoxysilane functionality, which induces cross-linking in the presence of water and $\mathrm{H}_{3} \mathrm{PO}_{4}$. However, the results suggest an absence of cross-linking with the PDMS. One advanced explanation is that the incompatibility between PEG and PDMS could lead to the gathering and collapsing of EO units around the cross-linking functional group. These phenomena would prevent the contact between the cross-linking function carried by the PEG and those present in the PDMS network, thus inhibiting the linking between these polymers.

Coatings containing the 45-Alk14-TMS and 45-Alk8-TMS additives showed similar behavior, with 8.4 and $9.0 \%$ weight loss. These compounds present reactive cross-linking functional groups like those of 6-TMS. The linker between the EO units and the trimethoxysilane group may protect the cross-linking ability against the collapsing of EO and the PEG cluster formation. However, it could be ineffective in avoiding the gathering of EO, which also decreases the accessibility between PEG and PDMS functions. This gathering seems to be confirmed by the domains visible in SEM images. For these products, we believe that the PEG chains collapse but do not end up surrounding the cross-linking group. Thus, to obtain efficient linking, the TMS group, by using a linker and by compatibilizing 
the hydrophilic additives with the PDMS matrix, should remain protected from both PEG collapsing and gathering.

PDMS $_{13}$ coatings formulated with PDMS-SiOH and PDMS-TMS additives resulted in very low weight losses of 2.8 and $3.2 \%$, respectively. This was unexpected, as these values were even lower than that of the PDMS reference (4.7\%). This indicates that these additives can be efficiently linked to the network and improve the cross-linking of the network itself. One possible explanation is that the presence of EO units on a very fluid copolymer increases the compatibility between ethanol and PDMS, and facilitates chain mobility and overall cross-linking. Liquid copolymers might act as lubricants that facilitate the mobility and cross-linking of the PDMS chains. PDMS linkers to small PEG chains (EO units < 13) combined with a linking function yield good linking properties in the PDMS network. The coatings prepared with PDMS $_{28}$ showed similar trends, and, again, coatings prepared with PDMS-SiOH and PDMS-TMS additives showed the lowest weight loss. However, each coating prepared with PDMS $_{28}$ had larger amounts of weight loss compared to their respective PDMS $_{13}$ variants.

\subsection{Contact Angle Measurements}

The amphiphilic character of the coating comes from the ability of the hydrophilic additives to migrate to the coating surface. This mobility could be provided by a high compatibilization between PDMS and the additives. The size of the additive should be a strong determinant as well. For non-cross-linkable additives, a low number of repeat units can provide high mobility. In contrast, linkable additives with a higher and sufficient number of repeat units will allow the attached chain to more easily access the surface. Contact angle results are shown in Figure 5 and demonstrate two main behaviors. The PDMS reference has an initial contact angle of $94^{\circ} \pm 2$, followed by $91^{\circ} \pm 3$ and $88^{\circ} \pm 3$ at 15 and $30 \mathrm{~s}$, respectively. Five other coatings presented similarly compared to the PDMS reference: 9-OH, 6-TMS, 45-OH, 45-Alk8-TMS, and 45-Alk14-TMS (Figure 5a,b). Two things must be noted about these coatings: (1) The contact angle at $t=0$ does not decrease, which means that there are probably no EO units at the surface. (2) The contact angles at $t=15 \mathrm{~s}$ and $t=30 \mathrm{~s}$ do not decrease significantly (with respect to PDMS reference), which could mean that the EO units cannot access the surface during this period of time. The most likely cause for the absence of EO units at the surface is that the hydrophilic glass slide may attract the PEG chains during the cross-linking. Furthermore, the lack of compatibility between these products and the PDMS matrix might restrain the chain mobility and hence the surface access.

Concerning the 45-Alk8-TMS and 45-Alk14-TMS coatings, the linking is slightly increased as demonstrated above and the surface access may be enhanced due to the length of the PEG chains. However, the $45 \mathrm{EO}$ units may collapse and be entangled in the PDMS matrix, limiting their mobility and the surface accessibility. Equivalent systems with lower number of EO units have shown good surface access and decreased contact angles $[15,23]$. Shorter PEG chains $(E O<13)$ should be used with $\mathrm{C}_{8}-\mathrm{C}_{14}$ linkers and linking functionality to obtain efficient surface accessibility.

Coatings containing PDMS-SiOH and PDMS-TMS additives had lower initial contact angles followed by a noticeable decrease. The coatings prepared with PDMS-SiOH and PDMS-TMS additives seem thus to have $\mathrm{EO}$ units present on the surface, even at $t=0$, demonstrating the ability of the hydrophilic additive to access the surface and increase the hydrophilicity. This may indicate that the hydrophilic additive is compatible and well dispersed within the PDMS matrix and does not accumulate particularly inside or at the bottom of the coatings. The decreasing contact angle over time indicates that the chains have sufficient mobility provided by the good compatibilization between these additives and the silicone matrix. The PEG chains collapsing does not seem to be an issue for these additives, as it decreases neither the mobility nor the surface accessibility. PDMS linkers increase the compatibility and the dispersion of the additive. The additive mobility after cross-linking seems to support this due to the combination of PDMS linkers and the small PEG chains, which do not cause detrimental entanglement. 

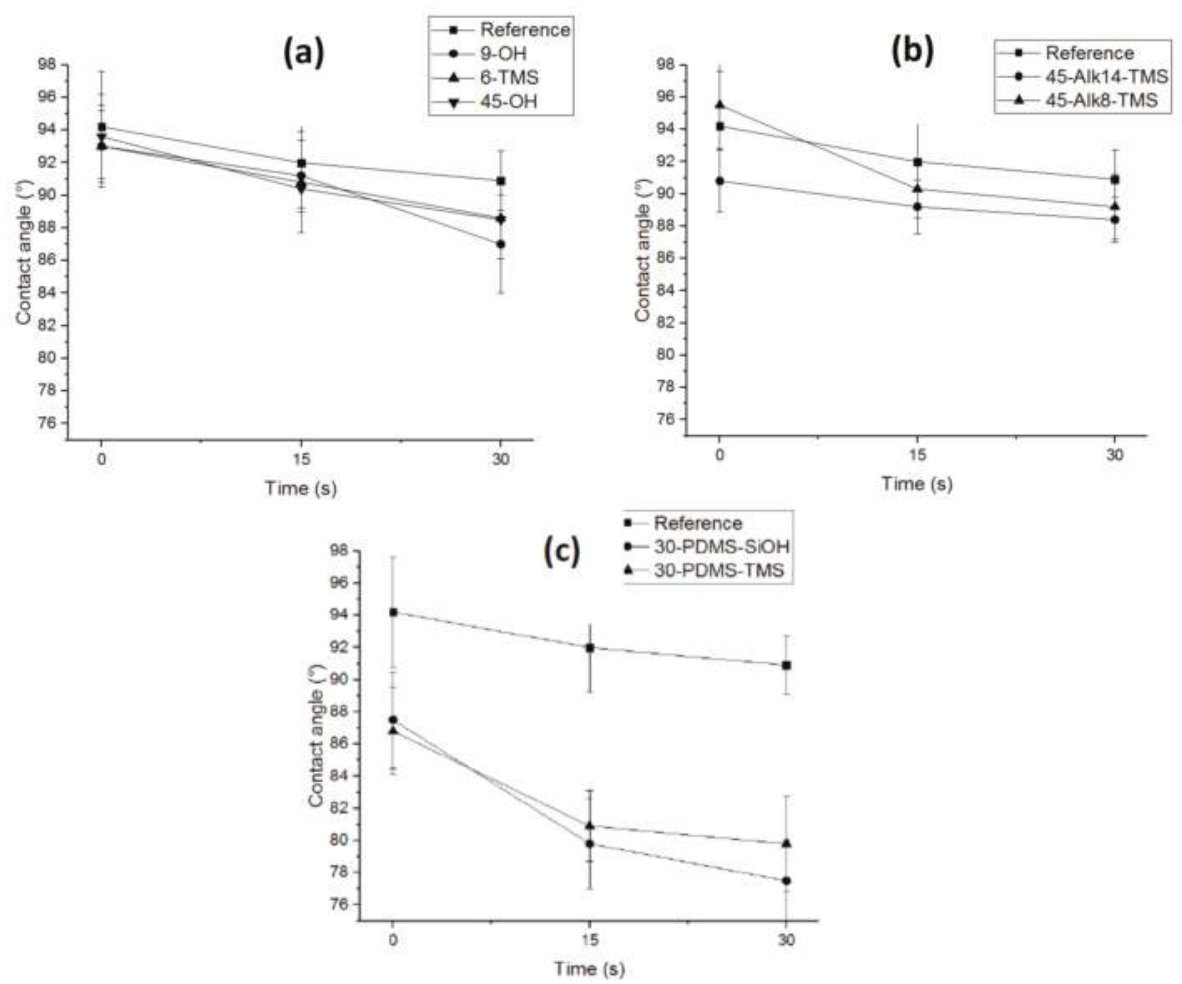

Figure 5. All hydrophilic additives were incorporated in the PDMS $_{13}$-matrix-ended methoxy. (a) Water contact angle of hydrophilic additives with no linker. (b) Contact angle of hydrophilic additives with linker alkyl. (c) Contact angle of PEG additives with linker PDMS.

All results presented above concern coatings prepared with PDMS $_{13}$. Compared to their PDMS $_{13}$ variants, coatings prepared with $\mathbf{P D M S}_{28}$ generally had higher initial contact angles $\left(98^{\circ} \pm 2.1\right)$ and presented less noticeable decreases of its contact angle over time. The coatings prepared with $\mathbf{P D M S}_{\mathbf{2 8}}$ presented less homogeneous surfaces, reduced linking ability, and less surface accessibility. This might be explained by the catalyst-solvent system, which may be suitable for PDMS $_{13}$ but inappropriate for PDMS $_{28}$. In this study, to obtain comparable results, all conditions were fixed and are the same for each coating (the amount of solvent, the amount of catalyst, the type of catalyst, the amount of additive, and the time of cross-linking) (Table S1).

\section{Discussion}

According to the results, a combination of three elements is needed to achieve an amphiphilic non-leachable and homogeneous surface: the number of EO units (PEG molecular weight), the presence of linkable groups, and the nature of the linker used. The first element, EO units, is critical toward achieving a homogeneous surface and with an amphiphilic character. Where the number of EO units was greater than 13, two issues could be observed: (1) the solidification of PEG during drying resulted in a heterogeneous surface, and (2) the gathering and collapsing of EO units around the linking group limited the bonding. Even with cross-linking functionality and an alkyl linker, the PEG additives 45-Alk8-TMS and 45-Alk14-TMS did not bond significantly with the network. A longer or more compatible linker seems necessary and/or the use of a lower molecular weight PEG. Indeed, a lower molecular weight PEG chain allows for the additive to be in a liquid state at room temperature 
$\left(20^{\circ} \mathrm{C}\right)$ [23], which facilitates the blending and drying of the film. Furthermore, lower molecular weight PEG can better access the surface. According to the results of the 6-TMS coating, the addition of a linking group seems necessary but does not achieve sufficient linking and surface accessibility. Our hypothesis is that the EO units collapse around the linking group, rendering it ineffective. In addition, the surface accessibility seems to require a good compatibilization. A linker must be added between the linking group and the EO units to improve compatibilization of the additive. The use of a PDMS linker provides a good compatibilization between both polymers. The addition of this linker appears to prevent collapse of EO units and enable cross-linking, as evidenced by the soxhlet results. The compatibilization also seems good (SEM) as well as the surface accessibility. Both the PDMS-TMS and PDMS-SiOH coatings presented qualities of good amphiphilic, non-leachable, and homogeneous additives. The main leverage for homogeneity seems to be the number of EO units in the PEG chain. For the accessibility, the nature of the linker is most important. For the linking, the size of the linker and its nature are critical. PDMS-TMS and PDMS-SiOH are good additive candidates with all the desired properties (homogeneity, accessibility, and linking).

\section{Conclusions}

In this study, the compatibilization of different PEG additives in a PDMS matrix was studied. This compatibilization is a critical parameter in achieving a formation of non-leaching coatings. Different properties such as linking ability of the additive in the matrix, surface accessibility, and coating homogeneity have been observed and discussed. It is suggested that the most important element is the presence of a reactive linking group that maintains the additive in the film. The availability of these groups depends on the presence of a linker that prevents the hindrance of the reactive chain end by EO groups. Another important element is the number of consecutive EO units. To obtain a homogeneous surface and promote surface accessibility, the additives should have a small number of EO units (under approximately 13 consecutive EO units) so that they remain liquid at room temperature and prevent disruption of the PDMS network. A third key factor is the compatibility provided by the linker. This compatibility contributes to the achievement of a homogeneous surface and can boost the surface accessibility of the EO units.

The best coatings were obtained with the copolymers PDMS-SiOH and PDMS-TMS, which contain a cross-linking group, a PDMS linker, and small pendant PEG chains. The linking group assures the linking to the PDMS network. The PDMS linker acts as a spacer to keep the linking active and as a compatibilizer due to its great affinity with the PDMS matrix. This kind of structure therefore has a high potential as an additive for non-leaching amphiphilic coatings.

Supplementary Materials: The supplementary materials are available online at http://www.mdpi.com/2073$4360 / 10 / 4 / 445 / s 1$.

Acknowledgments: The National Agency of Research (ANR) and the South Britany University (UBS) are gratefully acknowledged.

Author Contributions: Karine Réhel, Fabienne Faÿ and Isabelle Linossier designed the research strategy; Guillaume Gillet and Fabrice Azemar performed the experiments, analyzed data and wrote the paper. All the authors took part in fruitful discussions during paper preparation.

Conflicts of Interest: The authors declare no conflict of interest. The founding sponsors had no role in the design of the study; in the collection, analyses, or interpretation of data; in the writing of the manuscript; or in the decision to publish the results. 


\section{References}

1. Callow, M.E.; Fletcher, R.L. The Influence of Low Surface Energy Materials on Bioadhesion-A Review. Int. Biodeterior. Biodegrad. 1994, 34, 333-348. [CrossRef]

2. Ng, J.M.K.; Gitlin, I.; Stroock, A.D.; Whitesides, G.M. Components for Integrated Poly(Dimethylsiloxane) Microfluidic Systems. Electrophoresis 2002, 23, 3461-3473. [CrossRef]

3. Zhang, H.; Chiao, M. Anti-Fouling Coatings of Poly(Dimethylsiloxane) Devices for Biological and Biomedical Applications. J. Med. Biol. Eng. 2015, 35, 143-155. [CrossRef] [PubMed]

4. Abbasi, F.; Mirzadeh, H. Adhesion between Modified and Unmodified Poly(Dimethylsiloxane) Layers for a Biomedical Application. Int. J. Adhes. Adhes. 2004, 24, 247-257. [CrossRef]

5. Mata, A.; Fleischman, A.J.; Roy, S. Characterization of Polydimethylsiloxane (PDMS) Properties for Biomedical Micro/Nanosystems. Biomed. Microdevices 2005, 7, 281-293. [CrossRef] [PubMed]

6. Mahadik, S.A.; Pedraza, F.; Mahadik, S.S.; Relekar, B.P.; Thorat, S.S. Biocompatible Superhydrophobic Coating Material for Biomedical Applications. J. Sol-Gel Sci. Technol. 2017, 81, 791-796. [CrossRef]

7. Martin, S.; Bhushan, B. Transparent, Wear-Resistant, Superhydrophobic and Superoleophobic Poly(Dimethylsiloxane) (PDMS) Surfaces. J. Colloid Interface Sci. 2017, 488 (Suppl. C), 118-126. [CrossRef] [PubMed]

8. Subramaniam, A.; Sethuraman, S. Chapter 18-Biomedical Applications of Nondegradable Polymers. In Natural and Synthetic Biomedical Polymers; Kumbar, S.G., Laurencin, C.T., Deng, M., Eds.; Elsevier: Oxford, UK, 2014; pp. 301-308.

9. Sundaram, H.S.; Cho, Y.; Dimitriou, M.D.; Weinman, C.J.; Finlay, J.A.; Cone, G.; Callow, M.E.; Callow, J.A.; Kramer, E.J.; Ober, C.K. Fluorine-Free Mixed Amphiphilic Polymers Based on PDMS and PEG Side Chains for Fouling Release Applications. Biofouling 2011, 27, 589-602. [CrossRef] [PubMed]

10. Truby, K.; Wood, C.; Stein, J.; Cella, J.; Carpenter, J.; Kavanagh, C.; Swain, G.; Wiebe, D.; Lapota, D.; Meyer, A.; et al. Evaluation of the performance enhancement of silicone biofouling-release coatings by oil incorporation. Biofouling 2009, 15, 141-150. [CrossRef] [PubMed]

11. Kavanagh, C.J.; Swain, G.W.; Kovach, B.S.; Stein, J.; Darkangelo-Wood, C.; Truby, K.; Holm, E.; Montemarano, J.; Meyer, A.; Wiebe, D. The Effects of Silicone Fluid Additives and Silicone Elastomer Matrices on Barnacle Adhesion Strength. Biofouling 2003, 19, 381-390. [CrossRef] [PubMed]

12. Camós Noguer, A.; Olsen, S.M.; Hvilsted, S.; Kiil, S. Field Study of the Long-Term Release of Block Copolymers from Fouling-Release Coatings. Prog. Org. Coat. 2017, 112, 101-108. [CrossRef]

13. Narrainen, A.P.; Hutchings, L.R.; Ansari, I.; Thompson, R.L.; Clarke, N. Multi-End-Functionalized Polymers: Additives to Modify Polymer Properties at Surfaces and Interfaces. Macromolecules 2007, 40, 1969-1980. [CrossRef]

14. Faÿ, F.; Hawkins, M.L.; Réhel, K.; Grunlan, M.; Linossier, I. Non-Toxic, Anti-Fouling Silicones with Variable PEO-Silane Amphiphile Content. Green Mater. 2016, 4, 53-62. [CrossRef]

15. Ciriminna, R.; Bright, F.V.; Pagliaro, M. Ecofriendly Antifouling Marine Coatings. ACS Sustain. Chem. Eng. 2015, 3, 559-565. [CrossRef]

16. Yang, W.J.; Neoh, K.-G.; Kang, E.-T.; Teo, S.L.-M.; Rittschof, D. Polymer Brush Coatings for Combating Marine Biofouling. Prog. Polym. Sci. 2014, 39, 1017-1042. [CrossRef]

17. Camós Noguer, A.; Olsen, S.M.; Hvilsted, S.; Kiil, S. Diffusion of Surface-Active Amphiphiles in Silicone-Based Fouling-Release Coatings. Prog. Org. Coat. 2017, 106 (Suppl. C), 77-86. [CrossRef]

18. Wu, Z.; Hjort, K. Surface Modification of PDMS by Gradient-Induced Migration of Embedded Pluronic. Lab Chip 2009, 9, 1500-1503. [CrossRef] [PubMed]

19. Røn, T.; Javakhishvili, I.; Hvilsted, S.; Jankova, K.; Lee, S. Ultralow Friction with Hydrophilic Polymer Brushes in Water as Segregated from Silicone Matrix. Adv. Mater. Interfaces 2016, 3, 1500472. [CrossRef]

20. Li, J.; Xie, Z.; Wang, G.; Ding, C.; Jiang, H.; Wang, P. Preparation and Evaluation of Amphiphilic Polymer as Fouling-Release Coating in Marine Environment. J. Coat. Technol. Res. 2017, 14, 1237-1245. [CrossRef]

21. Nendza, M. Hazard Assessment of Silicone Oils (Polydimethylsiloxanes, PDMS) Used in Antifouling-/Foul-Release-Products in the Marine Environment. Mar. Pollut. Bull. 2007, 54, 1190-1196. [CrossRef] [PubMed]

22. Zhang, X.; Brodus, D.; Hollimon, V.; Hu, H. A Brief Review of Recent Developments in the Designs that Prevent Bio-Fouling on Silicon and Silicon-Based Materials. Chem. Cent. J. 2017, 11, 18. [CrossRef] [PubMed] 
23. Majumdar, R.; Alexander, K.S.; Riga, A.T. Physical Characterization of Polyethylene Glycols by Thermal Analytical Technique and the Effect of Humidity and Molecular Weight. Pharmazie 2010, 65, 343-347. [PubMed]

24. Dantas, L.C.; da Silva-Neto, J.P.; Dantas, T.S.; Naves, L.Z.; das Neves, F.D.; da Mota, A.S. Bacterial Adhesion and Surface Roughness for Different Clinical Techniques for Acrylic Polymethyl Methacrylate. Int. J. Dent. 2016, 2016, 8685796. [CrossRef] [PubMed]

25. Murthy, R.; Cox, C.D.; Hahn, M.S.; Grunlan, M.A. Protein-Resistant Silicones: Incorporation of Poly(ethylene oxide) via Siloxane Tethers. Biomacromolecules 2007, 8, 3244-3252. [CrossRef] [PubMed]

(C) 2018 by the authors. Licensee MDPI, Basel, Switzerland. This article is an open access article distributed under the terms and conditions of the Creative Commons Attribution (CC BY) license (http:/ / creativecommons.org/licenses/by/4.0/). 
Article

\title{
Synthesis, Characterization and Microstructure of New Liquid Poly(methylhydrosiloxanes) Containing Branching Units $\mathrm{SiO}_{4 / 2}$
}

\author{
Jerzy J. Chruściel ${ }^{1, *}$, Marzena Fejdyś ${ }^{2}$ and Witold Fortuniak ${ }^{3}$ \\ 1 Textile Research Institute, Brzezińska Str. 5/15, 92-103 Łódź, Poland \\ 2 The Institute of Security Technologies "MORATEX", Marii Skłodowskiej-Curie Str. 3, 90-505 Łódź, Poland; \\ mfejdys@moratex.eu \\ 3 Centre of Molecular and Macromolecular Studies, Polish Academy of Sciences, Sienkiewicza Str. 112, \\ 90-363 Łódź, Poland; wfortuni@cbmm.lodz.pl \\ * Correspondence: jchrusci@p.lodz.pl or jchrusciel@iw.lodz.pl; Tel.: +48-42-61-63-187; Fax: +48-42-67-92-638
}

Received: 28 March 2018; Accepted: 25 April 2018; Published: 28 April 2018

\begin{abstract}
Six liquid branched poly(methylhydrosiloxanes) of new random structures (PMHS-Q), containing quadruple branching units $\mathrm{SiO}_{4 / 2}(\mathrm{Q})$, both $\mathrm{MeHSiO}\left(\mathrm{D}^{\mathrm{H}}\right)$ and $\mathrm{Me}_{2} \mathrm{SiO}(\mathrm{D})$ chain building units (or only mers $\mathrm{MeHSiO}$ ), and terminal groups $\mathrm{Me}_{3} \mathrm{SiO}_{0.5}(\mathrm{M})$ were prepared by a hydrolytic polycondensation method of appropriate organic chlorosilanes and tetraethyl ortosilicate (TEOS), in diethyl ether medium at temperature below $0{ }^{\circ} \mathrm{C}$. Volatile low molecular weight siloxanes were removed by a vacuum distillation at $150-155{ }^{\circ} \mathrm{C}$. Yields of PMHS-Q reached from $55-69 \mathrm{wt} \%$. Their dynamic viscosities were measured in the Brookfield HBDV+IIcP cone-plate viscometer and ranged from 10.7-13.1 cP. Molecular weights (MW) of PMHS-Q $\left(\mathrm{M}_{\mathrm{n}}=2440-6310 \mathrm{~g} / \mathrm{mol}\right.$, $\left.\mathrm{M}_{\mathrm{W}}=5750-10,350 \mathrm{~g} / \mathrm{mol}\right)$ and polydispersities of $\mathrm{MW}\left(\mathrm{M}_{\mathrm{W}} / \mathrm{M}_{\mathrm{n}}=2.0-2.8\right)$ were determined by a size exclusion chromatography (SEC). All polymers were characterized by FTIR, ${ }^{1} \mathrm{H}$ - and ${ }^{29} \mathrm{Si}-\mathrm{NMR}$, and an elemental analysis. A microstructure of siloxane chains was proposed on a basis of ${ }^{29} \mathrm{Si}-\mathrm{NMR}$ results and compared with literature data.
\end{abstract}

Keywords: hyperbranched poly(methylhydrosiloxanes); hydrolytic polycondensation; ${ }^{29} \mathrm{Si}-\mathrm{NMR}$; topology of polysiloxane chains

\section{Introduction}

Poly(methylhydrosiloxanes) (PMHS) are inorganic-organic hybrid polymers with inorganic backbone, composed of alternatively bound silicon and oxygen atoms. Hydrogen atoms and methyl groups are the main substituents of silicon atoms in PMHS; however, other organic groups may be attached to silicon atoms in their structures as well. Most PMHS are colorless oils, though some of them are solids [1-9]. Linear, star, hyperbranched and dendritic poly(methylhydrosiloxanes), as well as spherical hydrosilicates, are important classes of functional silicones. Many methods of their preparation were described in our previous publication, concerning synthesis and characterization of branched PMHS containing triple branching units $\mathrm{MeSiO}_{1.5}$ (T) [9]. PMHS find numerous practical applications. Most often they are used as cross-linking agents in a technology of silicone elastomers [9-15].

One of the newer synthetic methods used for the preparation of poly(dimethylsiloxanes-co-methylhydrosiloxanes) (PDMS-co-PMHS) is based on an equilibration polymerization of poly(dimethylsiloxanes) with linear hydrosiloxane polymer and hexamethyldisiloxane $\mathrm{Me}_{3} \mathrm{SiOSiMe}_{3}$, towards phosphonitrile catalyst $\left[\mathrm{Cl}_{3} \mathrm{P}\left(\mathrm{NPCl}_{2}\right)_{2} \mathrm{PCl}_{3}\right]^{+} \mathrm{PCl}_{6}{ }^{-}$at elevated temperature [16-20].

A polymerization of 1,3,5,7-tetramethylcyclotetrasiloxane $\left(\mathrm{D}^{\mathrm{H}}{ }_{4}\right)\left[\mathrm{D}^{\mathrm{H}}=\mathrm{H}\left(\mathrm{CH}_{3}\right) \mathrm{SiO}\right]$ was carried out in the presence of the non-ionic emulgator $\mathrm{C}_{12} \mathrm{H}_{25}\left(\mathrm{OCH}_{2} \mathrm{CH}_{2}\right)_{23} \mathrm{OH}$ and dodecylbenzenesulphonic acid and it was accelerated by ultrasounds $[21,22]$. 
Weber and Paulasaari obtained poly(1-hydro-1,3,3,5,5-pentamethyltrisiloxane) with a regular chain structure by the polymerization of a new monomer, pentamethylcyclotrisiloxane $\mathrm{c}-\left(\mathrm{Me}_{2} \mathrm{SiO}\right)_{2}(\mathrm{MeHSiO})\left[\mathrm{c}-\mathrm{D}_{2} \mathrm{D}^{\mathrm{H}} ; \mathrm{D}=\left(\mathrm{CH}_{3}\right)_{2} \mathrm{SiO}\right]$ in tetrahydrofurane (THF), in the presence of $\mathrm{Ph}_{2} \mathrm{Si}(\mathrm{OLi})_{2}$, at $-79{ }^{\circ} \mathrm{C}[23]$. The starting monomer $\mathrm{c}-\mathrm{D}_{2} \mathrm{D}^{\mathrm{H}}$ was prepared by heterocondensation of $\mathrm{MeHSiCl}_{2}$ with $\mathrm{HO}\left(\mathrm{Me}_{2} \mathrm{SiO}\right)_{2} \mathrm{H}$ (yield: $49 \%$ ). At the same time was elaborated a new sequential polycondensation method ("one-pot") leading to three homological series of new PDMS- $b$-PMHS with a general formula:

$$
\mathrm{RMe}_{2} \mathrm{SiO}\left[\left(\mathrm{Me}_{2} \mathrm{SiO}\right)_{\mathrm{m}}(\mathrm{MeHSiO})_{\mathrm{k}}\right]_{\mathrm{n}}\left(\mathrm{Me}_{2} \mathrm{SiO}\right)_{\mathrm{m}} \mathrm{SiMe}_{2} \mathrm{R} \text {, }
$$

(where: $\mathrm{R}=-\mathrm{OH}$ or $-\mathrm{Me} ; \mathrm{m}=2,6,10,14, \sim 50 ; \mathrm{k}=1-4 ; \mathrm{n}=5,10,15,20$ ), containing single or multiple $\mathrm{MeHSiO}$ units in macromolecules $[9,24]$. This synthetic method is based on a non-stoichiometric polycondensation of appropriate dimethylsiloxane- $\alpha, \omega$-diols $\mathrm{HO}\left(\mathrm{Me}_{2} \mathrm{SiO}\right)_{\mathrm{m}} \mathrm{H}$ with siloxane oligomers of a general formula $\mathrm{Cl}(\mathrm{MeHSiO})_{\mathrm{k}-1} \mathrm{SiHMeCl}$, having chloro(hydro)silyl functionalities, followed by termination reactions with chlorotrimethylsilane $\mathrm{Me}_{3} \mathrm{SiCl}$, when PDMS- $b$-PMHS chains were blocked with $\left(\mathrm{CH}_{3}\right)_{3} \mathrm{SiO}_{0.5}(\mathrm{M})$ groups. An isolation and characterization of many model $\mathrm{H}$-siloxanes helped us to establish the regular microstructure of prepared PDMS- $b$-PMHS, which was further confirmed by ${ }^{1} \mathrm{H}$ - and ${ }^{29}$ Si-NMR studies $[9,25]$.

In recent years a growing interest has been observed in the field of syntheses of star, highly branched, and dendritic poly(methylhydrosiloxanes). Cage silsesquioxanes ("spherosiloxanes"): $\mathrm{T}^{\mathrm{H}} 8$, $\mathrm{T}^{\mathrm{H}}{ }_{10}, \mathrm{~T}^{\mathrm{H}}{ }_{12}, \mathrm{~T}^{\mathrm{H}}{ }_{14}$, and $\mathrm{T}_{16}^{\mathrm{H}}\left(\mathrm{T}^{\mathrm{H}}=\mathrm{HSiO}_{1.5}\right)$ are solids, which can be prepared by the hydrolytic polycondensation of: (a) trichlorosilane $\mathrm{HSiCl}_{3}$, carried out in the presence of $\mathrm{FeCl}_{3}$ in methanol medium [24,26-28], or (b) trimethoxysilane $\mathrm{HSi}\left(\mathrm{OCH}_{3}\right)_{3}$, saturated with anhydrous $\mathrm{HCl}$, in acetic acid solution [29-32], or in the presence of concentrated $\mathrm{H}_{2} \mathrm{SO}_{4}$ [33,34]. Octakis(dimethylsiloxy)octasilsesquioxane $\left[\left(\mathrm{HMe}_{2} \mathrm{SiO}\right) \mathrm{SiO}_{1.5}\right]_{8}\left[\mathrm{Q}_{8} \mathrm{M}_{8}^{\mathrm{H}}, \mathrm{Q}=\mathrm{SiO}_{4 / 2}, \mathrm{M}^{\mathrm{H}}=\mathrm{H}\left(\mathrm{CH}_{3}\right)_{2} \mathrm{SiO}_{0.5}\right]$ can be prepared with high yields in reaction of octakis(tetramethylammonium) octasilsesquioxane with $\mathrm{ClSiMe}_{2} \mathrm{H}$ [35-37], up to $85-91 \%$ yield [38,39]. Currently a low molecular mass star tetrakis(dimethylsiloxy)silane $\mathrm{Si}\left[\mathrm{OSi}\left(\mathrm{CH}_{3}\right)_{2} \mathrm{H}\right]_{4}$, octahydrosilsesquioxane $\left(\mathrm{T}^{\mathrm{H}}{ }_{8}\right)$ and cubic $\mathrm{Q}_{8} \mathrm{M}^{\mathrm{H}}{ }_{8}$ are commercially available [35,40-46].

Recently siloxane-polyhedral silsesquioxane copolymers (soluble in THF) were prepared by the dehydrogenative condensation of $\mathrm{T}^{\mathrm{H}}{ }_{8}$ with diphenylsilanediol, tetraphenyldisiloxane diol or oligodimethylsiloxane- $\alpha, \omega$-diols in the presence of diethylhydroxylamine, followed by trimethylsilylation $[47,48] . \mathrm{T}_{8}^{\mathrm{H}}$ was also applied as a precursor of mezoporous silica, which was prepared without using any template or surfactant [49].

An equilibration of octamethylcyclotetrasiloxane $\left[\left(\mathrm{Me}_{2} \mathrm{SiO}\right)_{4}, \mathrm{D}_{4}\right]$ with $\mathrm{Si}\left[\mathrm{OSi}\left(\mathrm{CH}_{3}\right)_{2} \mathrm{H}\right]_{4}$ and trifluoromethanesulphonic acid led to tetraarm star polysiloxane $\mathrm{Si}\left\{\left[\mathrm{OSi}\left(\mathrm{CH}_{3}\right)_{2}\right]_{n} \mathrm{OSi}\left(\mathrm{CH}_{3}\right)_{2} \mathrm{H}\right\}_{4}[50,51]$. Six- and eight-membered silicates: hexakis(dimethylsiloxy)cyclotrisiloxane $\left[\left(\mathrm{HMe}_{2} \mathrm{SiO}\right)_{2} \mathrm{SiO}_{3}\right.$ and octakis(dimethylsiloxy)cyclotetrasiloxane $\left[\left(\mathrm{HMe}_{2} \mathrm{SiO}\right)_{2} \mathrm{SiO}\right]_{4}$ were synthesized with low yields, from reactions of pyrolysis products of wollastonite. $\left[\left(\mathrm{HMe}_{2} \mathrm{SiO}_{2} \mathrm{SiO}_{3}\right.\right.$ was prepared in reaction of chlorodimethylsilane $\mathrm{HMe}_{2} \mathrm{SiCl}$ with pseudowollastonite $\mathrm{Ca}_{3} \mathrm{Si}_{3} \mathrm{O}_{9} \mathrm{Cl}_{6}$, while $\left[\left(\mathrm{HMe}_{2} \mathrm{SiO}\right)_{2} \mathrm{SiO}\right]_{4}$ was prepared by heating octakis(trimethylsiloxy)cyclotetrasiloxane $\left[\left(\mathrm{Me}_{3} \mathrm{SiO}\right)_{2} \mathrm{SiO}\right]_{4}$ with $1,1,3,3-$ -tetramethyldisiloxane $\mathrm{HMe}_{2} \mathrm{SiOSiMe}_{2} \mathrm{H}$ in the presence of trifluoromethanesulphonic acid [52,53]. The equilibration of $\left[\left(\mathrm{HMe}_{2} \mathrm{SiO}\right)_{2} \mathrm{SiO}_{3}\right.$ with $\mathrm{D}_{4}$ and triflic acid gave PMHS of the following branched structure: $-\left\{-\mathrm{OSi}\left[\left(\mathrm{OSiMe}_{2} \mathrm{O}\right)_{n} \mathrm{SiMe}_{2} \mathrm{H}_{2}-\right\}_{6}-[52]\right.$. The $\mathrm{Si}-\mathrm{H}$ terminated multifunctional silicone dendrimer, i.e., tetrakis(dimethylsiloxy)silane, was prepared with $69 \%$ yield by the reaction of TEOS and dimethoxysilane [54].

Zhang et al. synthesized polysilsesquioxanes of a ladder structure, containing units $\mathrm{HSiO}_{1.5}$ and $\mathrm{MeSiO}_{1.5}$, by hydrolysis of byproducts prepared through condensation of $\mathrm{HSiCl}_{3}$ and $\mathrm{MeSiCl}_{3}$ with p-phenylenediamine or ethylenediamine [55-57].

A silicone dendrimer of a third generation with symmetrical structure and the general formula $\left.\left(\mathrm{CH}_{3} \mathrm{SiO}_{1.5}\right)_{22}\left[\left(\mathrm{CH}_{3}\right)_{2} \mathrm{SiO}\right)\right]_{162}\left[\mathrm{H}\left(\mathrm{CH}_{3}\right)_{2} \mathrm{SiO}_{0.5}\right]_{24}$ was prepared by Masamune et al. [58] in a multistep synthesis from siloxane oligomers containing functional groups: $\mathrm{Si}-\mathrm{H}, \mathrm{Si}-\mathrm{Cl}, \mathrm{Si}-\mathrm{Br}$ and $\mathrm{Si}-\mathrm{OH}$. It had 24 terminal functional Si-H groups "on the surface". Branched resins, containing 10-14 
$\mathrm{Si}-\mathrm{H}$ functional groups in macromolecules were synthesized by the hydrolytic polycondensation of methyldichlorosilane with dimethyldichlorosilane, trimethylchlorosilane and methyltriethoxy)silane or phenyl(triethoxy)silane. These resins were used as crosslinking agents for addition of cured silicone elastomers [59]. Condensation of (triethoxy)silane $\mathrm{HSi}\left(\mathrm{OC}_{2} \mathrm{H}_{5}\right)_{3}$, towards $\mathrm{HCl}$ solution, in the mixture of THF and methylisobutyl ketone (MIBK), led to soluble multifunctional poly(hydrogensilsesquioxanes) (PHSSQ) of combined cage-like and network-like structures [60]. A solid four-membered silsesquioxane ring compound $\left(\mathrm{PhSiO}_{1.5}\right)_{8}(\mathrm{MeHSiO})_{2}$, so-called "double-deckershaped-silsesquioxane", was prepared from reaction of $\mathrm{MeHSiCl}_{2}$ with a byproduct, which was obtained via a condensation of phenyl(trimethoxy)silane and $\mathrm{NaOH}$ with $20 \%$ yield [61].

Twelve new liquid branched poly(methylhydrosiloxanes) with statistical structures (b-r-PMHS), containing triple branching units $\mathrm{MeSiO}_{1.5}(\mathrm{~T})$, both $\mathrm{Me}_{2} \mathrm{SiO}(\mathrm{D})$ and $\mathrm{MeHSiO}\left(\mathrm{D}^{\mathrm{H}}\right)$ chain building units (or only mers MeHSiO), and two b-r-PMHS containing five different structural units: $\mathrm{D}, \mathrm{D}^{\mathrm{H}}$, $\mathrm{T}$ and $\mathrm{T}^{\mathrm{H}}$ and trimethylsiloxy end groups $\mathrm{Me}_{3} \mathrm{SiO}_{0.5}(\mathrm{M})$ were prepared by the hydrolytic polycondensation method of appropriate chlorosilanes in diethyl ether medium at temperature $<0{ }^{\circ} \mathrm{C}$. Yields of b-r-PMHS ranged from 57-84 wt \% (after removal of low molecular weight oligosiloxanes by a vacuum distillation at $125-150{ }^{\circ} \mathrm{C}$ ). All polymeric products were characterized by FTIR, ${ }^{1} \mathrm{H}$ - and ${ }^{29} \mathrm{Si}-\mathrm{NMR}$, and elemental analysis. Their dynamic viscosities were very low and usually ranged from $\sim 8-30 \mathrm{cP}$, which presumably resulted from their globular structure [9].

Methyl-substituted silica gels with $\mathrm{Si}-\mathrm{H}$ functionalities were prepared by hydrolysis and condensation reactions of triethoxysilane and methyldiethoxysilane, used in various molar ratios [62]. They gave higher ceramic residue after pyrolysis than gels based only on $\mathrm{MeSiO}_{1.5}$ branching units [63].

In the present work, we describe the hydrolytic polycondensation synthetic route to new liquid branched poly(methylhydrosiloxanes) of random structures (PMHS-Q), containing both $\mathrm{MeHSiO}\left(\mathrm{D}^{\mathrm{H}}\right)$ and $\mathrm{Me}_{2} \mathrm{SiO}(\mathrm{D})$ chain building units (or only mers $\mathrm{MeHSiO}$ ), quadruple branching units $\mathrm{SiO}_{4 / 2}(\mathrm{Q}$ ), and terminal groups $\mathrm{Me}_{3} \mathrm{SiO}_{0.5}$, from appropriate organic chlorosilanes and tetraethoxysilane.

\section{Materials and Methods}

Dichloromethylsilane $\mathrm{MeHSiCl}_{2}$ (MDS, $99 \%$, b.p. $41^{\circ} \mathrm{C}$ ), dichlorodimethylsilane $\mathrm{Me}_{2} \mathrm{SiCl}_{2}$ (DDS, b.p. $70-71^{\circ} \mathrm{C}$ ), tetraethoxysilane $\mathrm{Si}\left(\mathrm{OC}_{2} \mathrm{H}_{5}\right)_{4}$ (b.p. $168^{\circ} \mathrm{C}$ ), (4-dimetylamino)pyridine (DMAP, $99 \%$ ) were all sourced from Aldrich Chemical Company Inc., USA. Chlorotrimethylsilane $\mathrm{Me}_{3} \mathrm{SiCl}$ was obtained from Fluka, Seelze, Germany (TMCS, $>99 \%$, b.p. $57^{\circ} \mathrm{C}$ ). Tetraethoxysilane $\mathrm{Si}(\mathrm{OEt})_{4}$ was obtained from Unisil, Tarnów, Poland (TEOS, $99 \%$, b.p. $168{ }^{\circ} \mathrm{C}$ ). Triethylamine $(>99 \%$, Fluka) was dried with anhydrous $\mathrm{KOH}$, decanted, and distilled over $\mathrm{P}_{2} \mathrm{O}_{5}$. Diethyl ether was purified and dried with anhydrous $\mathrm{KOH}$, and distilled over $\mathrm{CaH}_{2}$.

All products were analyzed by a nuclear magnetic resonance (NMR), infrared spectroscopy (FTIR) and gel chromatography (SEC). FTIR spectra (neat) were done on spectrophotometer IR Bio-Rad 175 C (American Laboratory Trading, East Lyme, CT 06333, USA) for samples placed between NaCI plates. ${ }^{1} \mathrm{H}-\mathrm{NMR}$ and ${ }^{29} \mathrm{Si}-\mathrm{NMR}$ (INEPT) spectra were recorded on Bruker DRX 500 machine (Bruker Physik AG, Karlsruhe, Germany) at CBMM PAN in Łódź. Hexamethyldisiloxane $\mathrm{Me}_{3} \mathrm{SiOSiMe}_{3}$ was used as an external standard in ${ }^{29} \mathrm{Si}-\mathrm{NMR}\left(\delta=6.98 \mathrm{ppm}\right.$, in $\left.\mathrm{CDCl}_{3}\right)$.

An elementary analysis $(\% \mathrm{C}$ and $\% \mathrm{H})$ was performed at the Centre of Molecular and Macromolecular Studies of the Polish Academy of Sciences in Łódź (CBMM PAN). The content of $\mathrm{Si}-\mathrm{H}$ groups was calculated from an integration ratio of their signals to $\mathrm{CH}_{3}$ signals in ${ }^{1} \mathrm{H}-\mathrm{NMR}$ spectra, and compared to theoretical integration ratios of $\mathrm{Si}-\mathrm{H}$ and $\mathrm{CH}_{3}$ signals. The content of $\mathrm{Si}$ was determined by the gravimetric method with $\mathrm{H}_{2} \mathrm{SO}_{4}$ (p.a.) [64].

Dynamic viscosities $\left(\eta^{25}\right)$ of polysiloxanes were measured at $25.0^{\circ} \mathrm{C}$ in a Brookfield cone-plate reoviscometer HBDV-II+cP (Brookfield Engineering Laboratories, Inc., Middleboro, MA 02346, USA), using a cone $\mathrm{CP} 40$.

The molecular masses and molecular mass distribution of polysiloxanes were analyzed by a size exclusion chromatography (SEC) in toluene solution, using LDC analytical chromatograph (Artisan 
Technology Group, Champaign, IL 61822, USA) equipped with refractoMonitor and a battery of two phenogel columns covering the MW range $10^{2}-10^{5} \mathrm{~g} \cdot \mathrm{mol}^{-1}$. Calibration was made with polystyrene Ultrastyrogel standards with MWs: $10^{2}, 10^{3}$, and $10^{4} \mathrm{~g} \cdot \mathrm{mol}^{-1}$.

Synthesis of Branched Polymethylhydrosiloxanes (PMHS-Q)

Branched polymethylhydrosiloxanes, containing only units $\mathrm{D}^{\mathrm{H}}$ and $\mathrm{Q}$, terminated with $\mathrm{Me}_{3} \mathrm{SiO}_{0.5}$ groups, with structures described by a general formula:

$$
\left(\mathrm{SiO}_{4 / 2}\right)_{\mathrm{y}}\left[\mathrm{CH}_{3}(\mathrm{H}) \mathrm{SiO}\right]_{\mathrm{n}}\left[\left(\mathrm{CH}_{3}\right)_{3} \mathrm{SiO}_{0.5}\right]_{\mathrm{p}}
$$

(where: $\mathrm{y}=1-3, \mathrm{n}=48-50, \mathrm{p}=2 \mathrm{y}+2$ ), and branched poly(dimethyl-co-methylhydro)siloxanes, containing both mers $\mathrm{D}$, as well mers $\mathrm{D}^{\mathrm{H}}$, units $\mathrm{Q}$ and end $\mathrm{Me}_{3} \mathrm{SiO}_{0.5}$ groups, of a general formula:

$$
\left(\mathrm{SiO}_{4 / 2}\right)_{\mathrm{y}}\left[\left(\mathrm{CH}_{3}\right)_{2} \mathrm{SiO}\right]_{\mathrm{m}}\left[\mathrm{CH}_{3}(\mathrm{H}) \mathrm{SiO}\right]_{\mathrm{n}}\left[\left(\mathrm{CH}_{3}\right)_{3} \mathrm{SiO}_{0.5}\right]_{\mathrm{p}}
$$

(where: $\mathrm{y}=1-3, \mathrm{~m}=\mathrm{n}=49-52, \mathrm{p}=2 \mathrm{y}+2$ ), were synthesized by the hydrolytic polycondensation of mixtures of tetraethoxysilane $\mathrm{Si}(\mathrm{OEt})_{4}$ and appropriate chlorosilanes: dichloromethylsilane $\mathrm{MeHSiCl}_{2}$, dichlorodimethylsilane $\mathrm{Me}_{2} \mathrm{SiCl}_{2}$, and chlorotrimethylsilane $\mathrm{Me}_{3} \mathrm{SiCl}$, in the medium of diethyl ether and water, at temperature ranged from $-10-0{ }^{\circ} \mathrm{C}$, within $3-5 \mathrm{~h}$. Molar ratios of chlorosilanes were changed, depending on expected molecular formula of polysiloxane. Amounts of substrates used in syntheses of branched PMHS-Q and times of additions of chlorosilanes are presented in Table 1. In the hydrolytic polycondensation reactions were used such amounts of distilled water, which were sufficient for a formation of hydrochloric acid with a final concentration about $20 \mathrm{wt} \%$.

Table 1. Amounts of substrates and a solvent, reaction time in syntheses of branched PMHS containing

\begin{tabular}{|c|c|c|c|c|c|c|c|}
\hline \multirow{3}{*}{ Substrates, solvent, and reaction conditions } & & \multicolumn{6}{|c|}{ Predicted molecular formulas of PMHS-Q * } \\
\hline & & 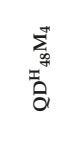 & 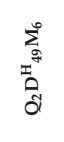 & $\begin{array}{l}\sum_{i^{\infty}}^{\infty} \\
x^{i n} \\
0 \\
0 \\
0\end{array}$ & 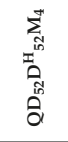 & 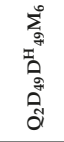 & $\begin{array}{l}\sum_{i}^{\infty} \\
x^{10} \\
0 \\
0 \\
0 \\
0 \\
0 \\
0\end{array}$ \\
\hline & & \multicolumn{6}{|c|}{ Amounts of reagents and a solvent } \\
\hline \multirow[b]{2}{*}{$\mathrm{Si}(\mathrm{OEt})_{4}$} & $(\mathrm{~mol})$ & 0.01 & 0.04 & 0.06 & 0.01 & 0.02 & 0.03 \\
\hline & $\left(\mathrm{cm}^{3}\right)$ & 2.2 & 8.9 & 13.4 & 2.2 & 4.6 & 6.7 \\
\hline \multirow[b]{2}{*}{$\mathrm{MeHSiCl}_{2}$ (MDS) } & $(\mathrm{mol})$ & 0.48 & 0.98 & 1.0 & 0.52 & 0.49 & 0.50 \\
\hline & $\left(\mathrm{cm}^{3}\right)$ & 50.0 & 102.0 & 104.1 & 54.1 & 51.5 & 52.1 \\
\hline \multirow[b]{2}{*}{$\mathrm{Me}_{2} \mathrm{SiCl}_{2}$ (DDS) } & $(\mathrm{mol})$ & - & - & - & 0.52 & 0.49 & 0.50 \\
\hline & $\left(\mathrm{cm}^{3}\right)$ & - & - & - & 63.1 & 59.8 & 60.6 \\
\hline \multirow{2}{*}{$\mathrm{Me}_{3} \mathrm{SiCl}(\mathrm{TMCS})$} & $(\mathrm{mol})$ & 0.04 & 0.12 & 0.16 & 0.04 & 0.06 & 0.08 \\
\hline & $\left(\mathrm{cm}^{3}\right)$ & 5.1 & 15.2 & 20.3 & 5.1 & 7.6 & 10.2 \\
\hline \multirow{2}{*}{$\mathrm{H}_{2} \mathrm{O}$} & $(\mathrm{mol})$ & 9.11 & 18.94 & 19.66 & 19.33 & 18.38 & 18.94 \\
\hline & $\left(\mathrm{cm}^{3}\right)$ & 164 & 341 & 354 & 348 & 331 & 341 \\
\hline \multirow{2}{*}{ (4-dimethylamino)-pyridine (DMAP) } & $(\mathrm{mol})$ & - & - & 0.003 & 0.0109 & 0.0108 & 0.01 \\
\hline & (g) & - & - & 0.3665 & 1.3316 & 1.3194 & 1.2217 \\
\hline \multirow{2}{*}{$\mathrm{Et}_{3} \mathrm{~N}$} & $(\mathrm{~mol})$ & - & - & 0.03 & 0.109 & 0.108 & 0.10 \\
\hline & $\left(\mathrm{cm}^{3}\right)$ & - & - & 4.2 & 101.2 & 15.1 & 13.9 \\
\hline Diethyl ether & $\left(\mathrm{cm}^{3}\right)$ & 50 & 80 & 60 & 100 & 80 & 90 \\
\hline Addition time of chlorosilanes and $\mathrm{Si}(\mathrm{OEt})_{4}$ & $(\min )$ & 50 & 60 & 95 & 80 & 55 & 95 \\
\hline Temperature during addition of chlorosilanes and $\mathrm{Si}(\mathrm{OEt})_{4}$ & $\left({ }^{\circ} \mathrm{C}\right)$ & $-4--2$ & $-1-3$ & $-1-3$ & $-1-3$ & $-1-6$ & $-2-2$ \\
\hline \multirow{2}{*}{$\begin{array}{l}\text { Stirring time after addition of chlorosilanes and } \mathrm{Si}(\mathrm{OEt})_{4} \\
\text { Drying of products: }\end{array}$} & $(\min )$ & 170 & 130 & 130 & 130 & 120 & 120 \\
\hline & & \multicolumn{3}{|c|}{ with anhydrous $\mathrm{MgSO}_{4}$} & \multicolumn{3}{|c|}{ by cooling in a fridge } \\
\hline
\end{tabular}
quadruple branching points, and methods of drying of products solutions.

* Average molecular composition of polymers, based on a stoichiometry of monomers. PMHS-Q: liquid branched poly(methylhydrosiloxanes) of random structures.

Reaction mixture was allowed to warm to room temperature within 120-170 min, acid layer was separated, and organosilicon layer was washed with water until neutral, transferred to an Erlenmayer flask, and dried at $\sim 4{ }^{\circ} \mathrm{C}$ with anhydrous magnesium sulfate overnight. Magnesium sulfate was filtered through Schott funnel G-3 and washed with ether. Alternatively, instead of drying with anhydrous 
$\mathrm{MgSO}_{4}$ traces of water were removed from products by cooling their ether solution in a refrigerator overnight, warming up the content of the flask to room temperature, and the ether solution of products was decanted from drops of water. The solvent was distilled off. In order to remove volatile cyclic and linear low molecular weight siloxane oligomers, the prepared products were heated at temperature $150-155^{\circ} \mathrm{C}$ under reduced pressure (16-21 mm Hg, 2128-2793 Pa), and subsequently under a vacuum (3-5 mm Hg, 400-665 Pa).

In a second step of syntheses of $\mathbf{Q}_{3} \mathrm{D}^{\mathrm{H}}{ }_{50} \mathbf{M}_{8}$ and other poly(dimethyl-co-methylhydro)siloxanes, containing both mers $\mathrm{D}$ and $\mathrm{D}^{\mathrm{H}}$, with a general formula:

$$
\left(\mathrm{SiO}_{4 / 2}\right)_{\mathrm{y}}\left[\left(\mathrm{CH}_{3}\right)_{2} \mathrm{SiO}\right]_{\mathrm{m}}\left[\mathrm{CH}_{3}(\mathrm{H}) \mathrm{SiO}\right]_{\mathrm{n}}\left[\left(\mathrm{CH}_{3}\right)_{3} \mathrm{SiO}_{0.5}\right]_{\mathrm{p}}
$$

(where: $\mathrm{y}=1-3, \mathrm{~m}=\mathrm{n}=49-52, \mathrm{p}=2 \mathrm{y}+2$ ), so called "extra blocking" of unreacted silanol groups $\mathrm{Si}-\mathrm{OH}$ was applied: in reactions with (chloro)trimethylsilane, in the presence of triethylamine, which was used as an acceptor of hydrogen chloride with $5 \%$ excess with respect to a stoichiometric amount. (4-Dimethylamino)pyridine (DMAP) was used as a nucleophilic catalyst in 1:10 mole ratio with respect to $\mathrm{Et}_{3} \mathrm{~N}$. Products untreated with extra amounts of TMCS and DMAP/Et $t_{3} \mathrm{~N}$ showed increase of their viscosity after few months and a presence of small drops of water from a homo- condensation reaction of residual $\mathrm{Si}-\mathrm{OH}$ groups.

The "extra blocking" reactions of silanol groups were carried out after drying step of ether solutions of products of the hydrolytic polycondensation, at room temperature within few hours. Precipitates of amines hydrochlorides were dissolved in diluted solution (5-10 wt \%) of hydrochloric acid, a water layers were discarded and washed with distilled water until neutral, dried with anhydrous $\mathrm{MgSO}_{4}$, and filtered. Ether was distilled off under atmospheric pressure and final products were evacuated under vacuum at temperature $150-155^{\circ} \mathrm{C}$ (Table 2). A chemical composition of volatile siloxanes was not analyzed.

Table 2. Yields of PMHS-Q, conditions of removal of volatile products, and results of measurements of dynamic viscosity of branched PMHS, containing quadruple branching units $Q$.

\begin{tabular}{|c|c|c|c|c|c|c|c|c|}
\hline \multicolumn{4}{|c|}{ PMHS-Q } & \multirow{3}{*}{$\begin{array}{c}\begin{array}{c}\text { Dynamic } \\
\text { viscosity }\end{array} \\
\text { (cP) }\end{array}$} & \multicolumn{2}{|c|}{ Evacuation conditions } & \multicolumn{2}{|c|}{ Volatile products } \\
\hline \multirow{2}{*}{\multicolumn{2}{|c|}{$\begin{array}{l}\text { Predicted molecular formula } \\
\text { (polymer abreviation) }\end{array}$}} & \multicolumn{2}{|c|}{ Yield } & & \multirow{2}{*}{$\begin{array}{c}\text { Bath temp. } \\
\left({ }^{\circ} \mathrm{C}\right)\end{array}$} & \multirow{2}{*}{$\begin{array}{l}\text { Time } \\
\text { (min) }\end{array}$} & \multirow{2}{*}{$\begin{array}{l}\text { B.p./pressure } \\
\left({ }^{\circ} \mathrm{C} / \mathrm{mm} \mathrm{Hg}\right)\end{array}$} & \multirow{2}{*}{$\begin{array}{c}\text { Mass } \\
\text { (g) }\end{array}$} \\
\hline & & (g) & $(w t \%)$ & & & & & \\
\hline $\mathrm{QD}_{48}^{\mathrm{H}} \mathrm{M}_{4}$ & (Q1) & 20.14 & 62 & 12.8 & 152 & 190 & $24 / 16-79 / 3.5$ & 9.27 \\
\hline$\widehat{\mathrm{Q}}_{2} \mathrm{D}^{\mathrm{H}}{ }_{49} \mathrm{M}_{6}$ & (Q2) & 48.70 & 68 & 11.0 & 154 & 200 & $23 / 16-77 / 3.5$ & 18.14 \\
\hline$\hat{\mathrm{Q}}_{3} \mathrm{D}^{\mathrm{H}}{ }_{50} \mathrm{M}_{8}$ & (Q3) & 52.82 & 69 & 13.1 & 155 & 190 & $23 / 18-70 / 3.5$ & 16.71 \\
\hline $\mathrm{QD}_{52} \mathrm{D}^{\mathrm{H}}{ }_{52} \mathrm{M}_{4}$ & (Q1D) & 40.29 & 55 & 12.5 & 150 & 210 & $21 / 21-80 / 5$ & 34.84 \\
\hline $\mathrm{Q}_{2} \mathrm{D}_{49} \mathrm{D}^{\mathrm{H}}{ }_{49} \mathrm{M}_{6}$ & (Q2D) & 40.49 & 56 & 11.8 & 155 & 200 & $27 / 19-78 / 4$ & 31.06 \\
\hline $\mathrm{Q}_{3} \mathrm{D}_{50} \mathrm{D}^{\mathrm{H}}{ }_{50} \mathrm{M}_{8}$ & (Q3D) & 43.77 & 58 & 10.7 & 155 & 190 & $21 / 16-74 / 4.5$ & 28.22 \\
\hline
\end{tabular}

\section{Results and Discussion}

\subsection{Synthesis of Branched Polymethylhydrosiloxanes (PMHS-Q)}

Syntheses of poly(methylhydrosiloxanes) with statistical and branched structures containing quadruple branching points $\mathrm{SiO}_{4 / 2}$ were carried out in the medium of diethyl ether at temperature below $0{ }^{\circ} \mathrm{C}$. Solutions of chlorosilanes and $\mathrm{Si}(\mathrm{OEt})_{4}$ in dry ether were added dropwise to water. In all syntheses were used such amounts of water which were necessary for hydrolysis reactions and dissolution of $\mathrm{HCl}$, allowing to obtain hydrochloric acid with concentrations approximately $20 \mathrm{wt} \%$.

Applying the hydrolytic polycondensation of mixtures of appropriate amounts of (tetraethoxy)-silane $\mathrm{Si}(\mathrm{OEt})_{4}$ and chlorosilanes: $\mathrm{MeHSiCl}_{2}, \mathrm{Me}_{2} \mathrm{SiCl}_{2}$, and $\mathrm{Me}_{3} \mathrm{SiCl}$, with water, at temperature from $-10-0{ }^{\circ} \mathrm{C}$, within $3-5 \mathrm{~h}$, were prepared branched poly(methylhydrosiloxanes) with $\mathrm{SiO}_{4 / 2}$ branching points and the general formula:

$$
\left[\mathrm{SiO}_{4 / 2}\right]_{\mathrm{y}}\left[\mathrm{CH}_{3}(\mathrm{H}) \mathrm{SiO}\right]_{\mathrm{n}}\left[\left(\mathrm{CH}_{3}\right)_{3} \mathrm{SiO}_{0.5}\right]_{\mathrm{p}}
$$


(where: $\mathrm{y}=1-3, \mathrm{n}=48-50, \mathrm{p}=2 \mathrm{y}+2$ ), containing quadruple branching points $\mathrm{SiO}_{4 / 2}(\mathrm{Q})$, mers $\mathrm{D}^{\mathrm{H}}$ and terminal groups $\mathrm{Me}_{3} \mathrm{SiO}_{0.5}$. Similarly, branched poly(dimethyl-co-methylhydro)siloxanescontaining branching units $\mathrm{Q}$, linear building blocks $\mathrm{D}$, and $\mathrm{D}^{\mathrm{H}}$, and terminal groups $\mathrm{M}$, were synthesized with the general formula:

$$
\left[\mathrm{SiO}_{4 / 2}\right]_{\mathrm{y}}\left[\left(\mathrm{CH}_{3}\right)_{2} \mathrm{SiO}\right]_{\mathrm{m}}\left[\mathrm{CH}_{3}(\mathrm{H}) \mathrm{SiO}\right]_{\mathrm{n}}\left[\left(\mathrm{CH}_{3}\right)_{3} \mathrm{SiO}_{0.5}\right]_{\mathrm{p}}
$$

where $\mathrm{y}=1-3, \mathrm{~m}=\mathrm{n}=49-52, \mathrm{p}=2 \mathrm{y}+2$. After addition of substrates stirring of obtained reaction mixtures was continued within next $2-3 \mathrm{~h}$, in order to reach full conversion of substrates and full hydrolysis of $\mathrm{Si}-\mathrm{Cl}$ and $\mathrm{Si}_{-}-\mathrm{OC}_{2} \mathrm{H}_{5}$ groups. In the case of syntheses of Q3, Q1D, Q2D, and Q3D termination reactions (so called "extra blocking" reactions) of unreacted silanol groups $\mathrm{Si}-\mathrm{OH}$ in reactions with (chloro)trimethylsilane were applied, in the presence of: (1) triethylamine as the acceptor of hydrogen chloride (used with $\sim 5-10 \%$ excess with respect to stoichiometric amounts); and (2) (4-dimethylamino)pyridine (DMAP) as the nucleophilic catalyst (used in 1:10 mole ratio with respect to $\mathrm{Et}_{3} \mathrm{~N}$ ).

Products not treated with additional amounts of TMCS and DMAP/Et $\mathrm{E}_{3} \mathrm{~N}$ showed increase of their viscosity after few months and a presence of traces of water, which could originate from the homocondensation reaction of residual $\mathrm{Si}-\mathrm{OH}$ groups. However, in the case of syntheses of $\mathbf{Q 1}$ and Q2 "extra blocking" was not applied, and no increase of their viscosity was observed during longer storage of these PMHS-Q. Ether solutions of products Q1, Q2, and Q3 were dried with anhydrous $\mathrm{MgSO}_{4}$, while polymers Q1D, Q2D, and Q3D were dried by freezing traces of water in the refrigerator overnight. Yields of prepared PMHS-Q ranged from 55-69 $\mathrm{wt} \%$ (Table 2). The highest yield was obtained for Q3.

The chemical structures of all PMHS-Q were confirmed by spectroscopic methods: FTIR and NMR $\left({ }^{1} \mathrm{H}\right.$ and ${ }^{29} \mathrm{Si}$ ) and the elemental analysis $(\% \mathrm{C}, \% \mathrm{H}$, and \% Si) (see Table 3 ).

Dynamic viscosities $\left(\eta^{25}\right)$ of PMHS-Q containing quadruple branching points $\mathrm{SiO}_{4 / 2}$, were very low and ranged from 10.7-13.1 cP. Low viscosities of PMHS-Q in comparison with linear polysiloxanes having similar molecular weights presumably may result from a globular structure of hyperbranched macromolecules. It is commonly known from a literature that dendrimers and hyperbranched polymers in solution and in melt have low viscosities. Their viscosities and molecular weights are much lower than those for linear analogs and depend on a degree of branching, a polarity of a solvent, a kind of functional group on their "surface", and also on $\mathrm{pH}$ of a polymer solution. Dendritic and hyperbranched polymers have a variable hydrodynamic radii depending on the property of solvents; they are smaller than those of their linear analogs with the same molar mass.

The values of molecular weights of prepared PMHS-Q determined by SEC method were lower than calculated values for predicted molecular formulas: $\mathrm{QD}_{52} \mathrm{D}_{52}^{\mathrm{H}} \mathrm{M}_{4}, \mathrm{Q}_{2} \mathrm{D}_{49} \mathrm{D}^{\mathrm{H}}{ }_{49} \mathrm{M}_{6}$, and $\mathrm{Q}_{3} \mathrm{D}_{50} \mathrm{D}_{50}^{\mathrm{H}} \mathrm{M}_{8}$. A polydispersity of molecular weights of PMHS-Q ranged from 2.0 to 2.8 . The molecular weights of dendrimers and hyperbranched polymers determined by SEC using polystyrene standards are regarded with some scepticism. The hydrodynamic radii were also susceptible to the polarity of functional groups on the periphery [65-67]. Values of $M_{n}$ and $M_{w}$ determined by SEC method with polystyrene standards for hyperbranched polysiloxanes were much lower than MW obtained with application of MALLS detectors [68-70].

Köhler et al. used the SEC, ${ }^{1} \mathrm{H}$ - and ${ }^{29} \mathrm{Si}$ NMR, and MALDI-TOF-MS methods for characterization of a linear poly(dimethylsiloxane)-co-poly(hydromethysiloxane) (PDMS-co-PHMS) copolymer with respect to chain length distribution, heterogeneity of chemical composition, and sequence distribution [71]. 
Table 3. Characteristics of polymethylhydrosiloxanes with branched, random structure of siloxane chains, containing branching units $Q$.

\begin{tabular}{|c|c|c|c|c|c|c|c|c|c|c|}
\hline \multirow{2}{*}{ PMHS-Q } & \multirow{2}{*}{$\mathbf{M}_{\mathrm{n}}$ (calc.) } & \multirow{2}{*}{$\mathbf{M}_{\mathbf{n}}$} & \multirow{2}{*}{$\mathbf{M}_{\mathbf{w}}$} & \multirow{2}{*}{$\mathbf{M}_{\mathbf{w}} / \mathbf{M}_{\mathbf{n}}$} & \multicolumn{2}{|c|}{$\% \mathrm{C}$} & \multicolumn{2}{|c|}{$\% \mathrm{H}$} & \multicolumn{2}{|c|}{$\% \mathrm{Si}$} \\
\hline & & & & & calc. & found & calc. & found & calc. & found \\
\hline $\mathrm{QD}_{48}^{\mathrm{H}} \mathrm{M}_{4}$ & 3271 & 6310 & 17,750 & 2.81 & 22.03 & $\begin{array}{l}21.51 \\
21.76\end{array}$ & 7.03 & $\begin{array}{l}6.61 \\
6.90\end{array}$ & 45.50 & 44.87 \\
\hline $\mathrm{Q}_{2} \mathrm{D}^{\mathrm{H}}{ }_{49} \mathrm{M}_{6}$ & 3554 & 3220 & 8330 & 2.59 & 22.64 & $\begin{array}{l}22.01 \\
22.11\end{array}$ & 7.09 & $\begin{array}{l}6.92 \\
7.01\end{array}$ & 45.05 & 45.15 \\
\hline $\mathrm{Q}_{3} \mathrm{D}^{\mathrm{H}}{ }_{50} \mathrm{M}_{8}$ & 3836 & 3840 & 10,350 & 2.69 & 23.16 & $\begin{array}{l}22.03 \\
22.14\end{array}$ & 7.14 & $\begin{array}{l}7.02 \\
6.85\end{array}$ & 44.65 & 43.81 \\
\hline $\mathrm{QD}_{52} \mathrm{D}^{\mathrm{H}}{ }_{52} \mathrm{M}_{4}$ & 7367 & 2650 & 6280 & 2.36 & 27.39 & $\begin{array}{l}28.89 \\
28.70\end{array}$ & 7.60 & $\begin{array}{l}7.89 \\
8.03\end{array}$ & 41.55 & 41.54 \\
\hline $\mathrm{Q}_{2} \mathrm{D}_{49} \mathrm{D}^{\mathrm{H}}{ }_{49} \mathrm{M}_{6}$ & 7187 & 2440 & 5750 & 2.35 & 27.57 & $\begin{array}{l}28.15 \\
28.40\end{array}$ & 7.63 & $\begin{array}{l}7.69 \\
7.88\end{array}$ & 41.42 & 41.40 \\
\hline $\mathrm{Q}_{3} \mathrm{D}_{50} \mathrm{D}^{\mathrm{H}}{ }_{50} \mathrm{M}_{8}$ & 7544 & 5100 & 10,210 & 2.00 & 27.07 & $\begin{array}{l}27.78 \\
27.92\end{array}$ & 7.65 & $\begin{array}{l}8.35 \\
7.98\end{array}$ & 41.32 & 41.30 \\
\hline
\end{tabular}

\subsection{Characterization of PMHS-Q by FTIR}

In all FTIR spectra of studied PMHS-Q containing quadruple branching points $Q$ were present absorption bands in the range $2160 \mathrm{~cm}^{-1}$, corresponding to stretching vibrations of Si-H bonds, and also absorption bands of the remaining groups of atoms: $\mathrm{Si}_{-}-\mathrm{CH}_{3}(2960,2890,1440,1400,1255$, and $\left.830-700 \mathrm{~cm}^{-1}\right)$, Si-O-Si $\left(1010-1110 \mathrm{~cm}^{-1}\right)$, and $\mathrm{Si}\left(\mathrm{CH}_{3}\right)_{3}\left(750,690 \mathrm{~cm}^{-1}\right.$ ) (see data in Table 4). Examples of the FTIR spectra of branched poly(methylhydrosiloxanes) are presented in Figures 1-3.

Table 4. Characteristic absorption bands in FTIR spectra of $\mathrm{Q}_{2} \mathrm{D}_{49} \mathrm{D}^{\mathrm{H}}{ }_{49} \mathrm{M}_{6}$.

\begin{tabular}{cccc}
\hline \multicolumn{2}{c}{ Wave number $\left[\mathrm{cm}^{-\mathbf{1}}\right]$} & Group or bond & Vibration \\
\hline found & literature data [72] & & \\
2965 & $2975-2950$ & $\mathrm{CH}_{3}$ & $v_{\text {asym } \mathrm{C}-\mathrm{H}}$ \\
2878 & $2885-2860$ & $\mathrm{CH}_{3}$ & $v_{\text {sym } \mathrm{C}-\mathrm{H}}$ \\
2164 & $2300-2100$ & $\mathrm{Si}-\mathrm{H}$ & $v$ Si-H \\
1450 & $1470-1420$ & $\mathrm{CH}_{3}$ & $\delta_{\text {asym } \mathrm{C}-\mathrm{H}}$ \\
1410 & $1390-1365$ & $\mathrm{CH}_{3}$ & $\delta_{\text {sym }} \mathrm{C}-\mathrm{H}$ \\
1260 & $1265-1250$ & $\mathrm{Si}-\mathrm{CH}_{3}$ & $\delta_{\text {asym }} \mathrm{Si}-\mathrm{C}$ \\
$1115-1027$ & $1100-1000$ & $\mathrm{Si}-\mathrm{O}-\mathrm{Si}$ & $v_{\text {asym }} \mathrm{Si}-\mathrm{O}$ \\
910 & $950-800$ & $\mathrm{Si}-\mathrm{H}$ & $\delta$ Si-H \\
864 & $860-750$ & $\mathrm{Si}-\mathrm{CH}$ & $v_{\text {asym }} \mathrm{Si}-\mathrm{C}$ \\
830 & $910-830$ & $\mathrm{Si}-\mathrm{O}$ & $v_{\text {asym }} \mathrm{Si}-\mathrm{O}$ \\
800 & 800 & $\mathrm{Si}-\mathrm{CH}_{3}$ & $\delta$ sym Si-C \\
759 & 755 & $\mathrm{Si}-\left(\mathrm{CH}_{3}\right)_{3}$ & $\delta_{\text {asym }} \mathrm{Si}-\mathrm{C}$ \\
\hline
\end{tabular}

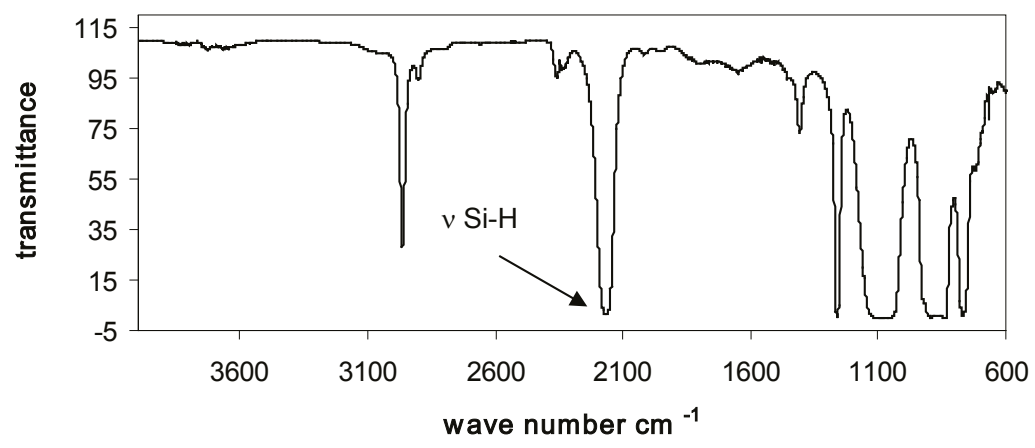

Figure 1. FTIR spectrum of $\mathrm{Q}_{2} \mathrm{D}^{\mathrm{H}}{ }_{49} \mathrm{M}_{6}$. 


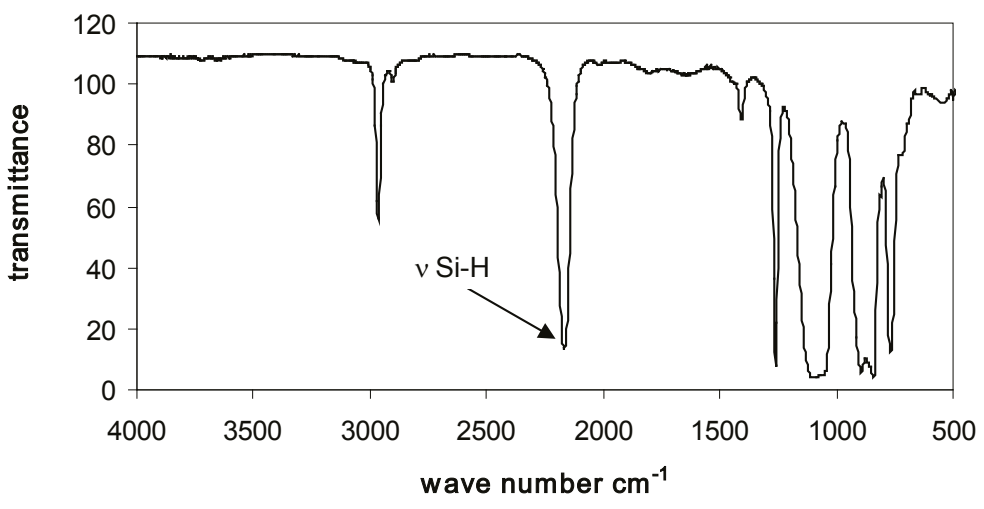

Figure 2. FTIR spectrum of $\mathrm{Q}_{3} \mathrm{D}^{\mathrm{H}}{ }_{50} \mathrm{M}_{8}$.

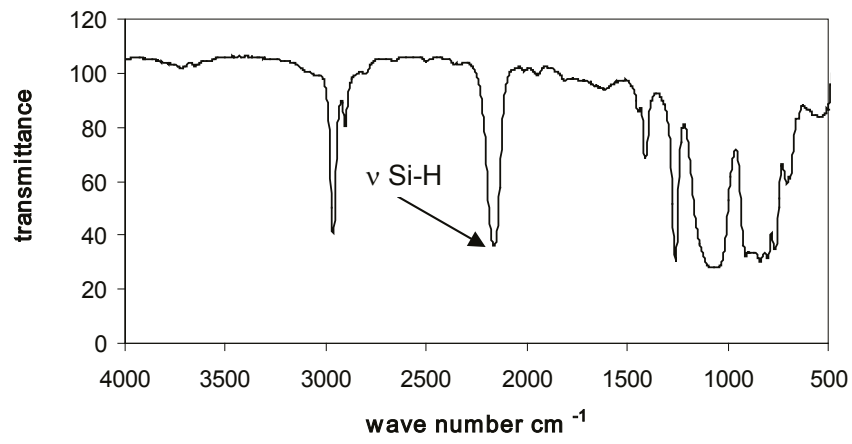

Figure 3. FTIR spectrum of $\mathrm{Q}_{2} \mathrm{D}_{49} \mathrm{D}^{\mathrm{H}}{ }_{49} \mathrm{M}_{6}$.

\subsection{Characterization of PMHS-Q by NMR}

In ${ }^{1} \mathrm{H}-\mathrm{NMR}$ spectra of copolymers, $\mathrm{QD}^{\mathrm{H}}{ }_{48} \mathbf{M}_{4}, \mathbf{Q}_{2} \mathrm{D}^{\mathrm{H}}{ }_{49} \mathrm{M}_{6}$ and $\mathrm{Q}_{3} \mathrm{D}^{\mathrm{H}}{ }_{50} \mathbf{M}_{8}$ were present signals at $\delta 0.01-0.22 \mathrm{ppm}$, corresponding to hydrogen atoms of $\mathrm{Si}_{-}-\mathrm{CH}_{3}$ groups and signals at $\delta$ about five parts per million, characteristic for hydrosilane groups $\mathrm{Si}-\mathrm{H}$. In the ${ }^{1} \mathrm{H}-\mathrm{NMR}$ spectra of copolymers: $\mathrm{QD}_{52} \mathrm{D}^{\mathrm{H}}{ }_{52} \mathrm{M}_{4}, \mathbf{Q}_{2} \mathrm{D}_{49} \mathrm{D}^{\mathrm{H}}{ }_{49} \mathrm{M}_{6}$, and $\mathrm{Q}_{3} \mathrm{D}_{50} \mathrm{D}^{\mathrm{H}}{ }_{50} \mathbf{M}_{8}$ were present signals at $\delta 0.0-0.30$ ppm, corresponding to hydrogen atoms of $\mathrm{Si}-\mathrm{CH}_{3}$ groups and signals at $\delta$ about five parts per million, characteristic for Si-H groups. Examples of the ${ }^{1} \mathrm{H}-\mathrm{NMR}$ and ${ }^{29} \mathrm{Si}-\mathrm{NMR}$ spectra of branched poly(methylhydrosiloxanes) are presented in Figures 4-7. 


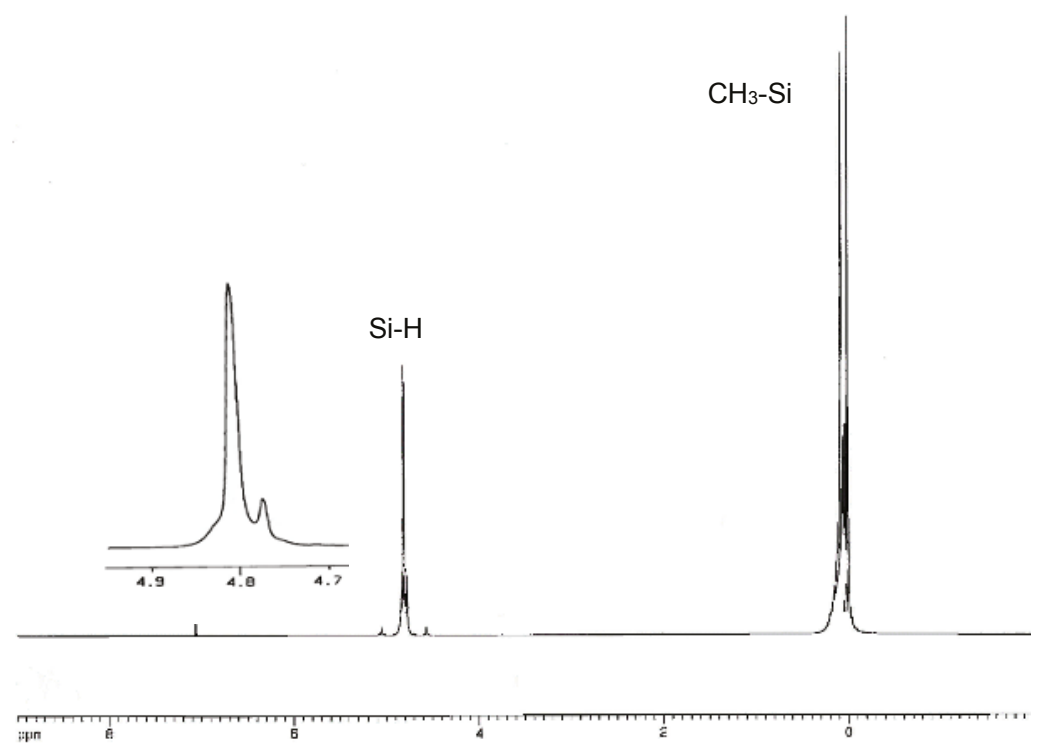

Figure 4. ${ }^{1} \mathrm{H}-\mathrm{NMR}$ spectrum (in $\mathrm{C}_{6} \mathrm{D}_{6}$ ) of polymethylhydrosiloxane $\mathrm{Q}_{3} \mathrm{D}^{\mathrm{H}}{ }_{50} \mathrm{M}_{8}$.

In ${ }^{29} \mathrm{Si}$-NMR INEPT spectra of copolymers $\mathbf{Q D}^{\mathrm{H}}{ }_{48} \mathbf{M}_{4}, \mathbf{Q}_{2} \mathrm{D}^{\mathrm{H}}{ }_{49} \mathbf{M}_{6}$ and $\mathbf{Q}_{3} \mathrm{D}^{\mathrm{H}}{ }_{50} \mathbf{M}_{8}$ were present signals at $\delta-31.62--39.97 \mathrm{ppm}$ corresponding to silicon atoms of units $\mathrm{D}^{\mathrm{H}}[8,9,54]$ and at $\delta$ 9.40-11.25 ppm corresponding to silicon atoms of end groups $\mathrm{Me}_{3} \mathrm{SiO}_{0.5}(\mathrm{M})$. In the range of $\delta$ $-63--68 \mathrm{ppm}$ in INEPT ${ }^{29} \mathrm{Si}-\mathrm{NMR}$ spectra were present signals of a very low intensity, from $\mathrm{Si}$ atoms of branching units $\mathrm{MeSiO}_{1,5}$, which could be formed during trace hydrolysis of $\mathrm{Si}-\mathrm{H}$ bonds. In the INEPT NMR spectra of copolymers $\mathbf{Q D}_{52} \mathrm{D}^{\mathrm{H}}{ }_{52} \mathbf{M}_{4}(\mathbf{Q} 1 \mathrm{D}), \mathbf{Q}_{2} \mathrm{D}_{49} \mathrm{D}^{\mathrm{H}}{ }_{49} \mathbf{M}_{6}(\mathrm{Q} 2 \mathrm{D})$, and $\mathbf{Q}_{3} \mathrm{D}_{50} \mathrm{D}^{\mathrm{H}}{ }_{50} \mathbf{M}_{8}$ (Q3D) existed signals at $\delta 7.27-9.92 \mathrm{ppm}$, corresponding to silicon atoms of end groups $\mathrm{Me}_{3} \mathrm{SiO}_{0.5}$ and at $\delta-34.61$ to $-38.87 \mathrm{ppm}$, characteristic for mers $\mathrm{MeHSiO}\left(\mathrm{D}^{\mathrm{H}}\right)$, and also at $\delta-18.77--21.85 \mathrm{ppm}$ from silicon atoms of units $\mathrm{Me}_{2} \mathrm{SiO}$ (D) [8,9,52]. It was impossible to observe signals of quadruple silicon atoms of units $\mathrm{SiO}_{4 / 2}$ in ${ }^{29} \mathrm{Si}-\mathrm{NMR}$ spectra, which were registered by the INEPT technique, so it was necessary to run ${ }^{29} \mathrm{Si}-\mathrm{NMR}$ spectra with application of the INVGATE program. A summary of chemical shifts data in the ${ }^{1} \mathrm{H}$ - and ${ }^{29} \mathrm{Si}-\mathrm{NMR}$ (INEPT and INVGATE) spectra of all PMHS-Q is presented in Table 5.

In the ${ }^{29}$ Si-NMR INVGATE spectra of branched random PMHS were present signals of silicon atoms corresponding to linear mers:

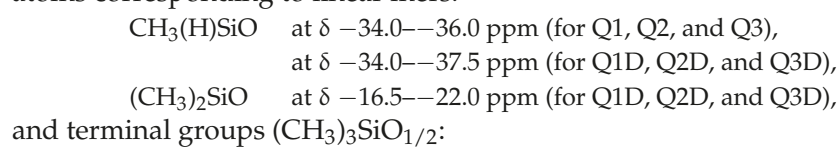

Resonance signals of $\mathrm{Si}$ atoms of branching units $\mathrm{Q}$ were present in the range of $\delta$ :

$$
-100.3--112.4 \mathrm{ppm}
$$

and they overlapped with very strong ${ }^{29} \mathrm{Si}$ signals of a borosilicate glass. 


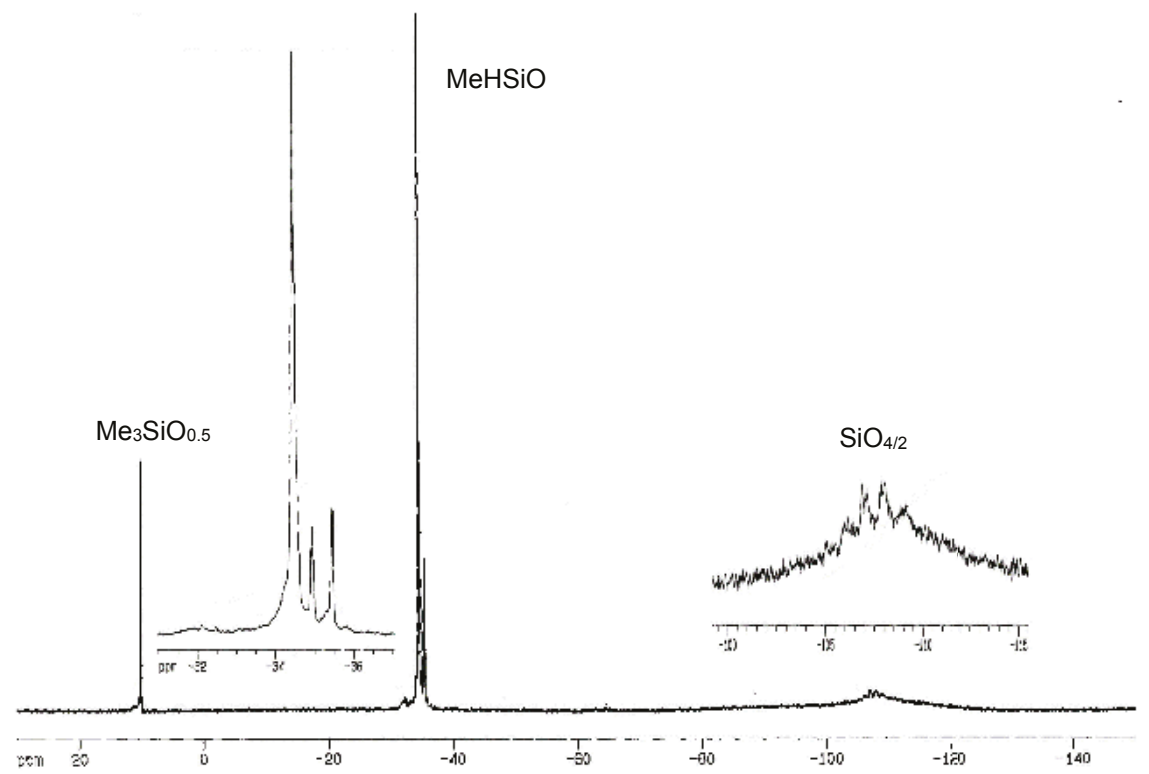

Figure 5. ${ }^{29} \mathrm{Si}-\mathrm{NMR}$ spectrum (INVGATE, in $\mathrm{C}_{6} \mathrm{D}_{6}$ ) of polymethylhydrosiloxane $\mathrm{Q}_{3} \mathrm{D}^{\mathrm{H}}{ }_{50} \mathrm{M}_{8}$.

In the ${ }^{29}$ Si-NMR INVGATE spectra of copolymers: $\mathrm{QD}^{\mathrm{H}}{ }_{48} \mathbf{M}_{4}, \mathbf{Q}_{2} \mathrm{D}^{\mathrm{H}}{ }_{49} \mathbf{M}_{6}$ and $\mathbf{Q}_{3} \mathrm{D}^{\mathrm{H}}{ }_{50} \mathbf{M}_{8}$ were present signals at: $\delta 9.40-11.25 \mathrm{ppm}$, corresponding to the terminal groups $\mathrm{Me}_{3} \mathrm{SiO}_{0.5}$, at $\delta$ $-31.81--36.25 \mathrm{ppm}$, characteristic for units $\mathrm{MeHSiO}\left(\mathrm{D}^{\mathrm{H}}\right)$, and also resonance signals in the range of $\delta-100.3--112.3 \mathrm{ppm}$ for Si atoms from units Q. According to data in the literature [73] chemical shifts of Si atoms from units Q exist in the range of $\delta-100--106 \mathrm{ppm}$.

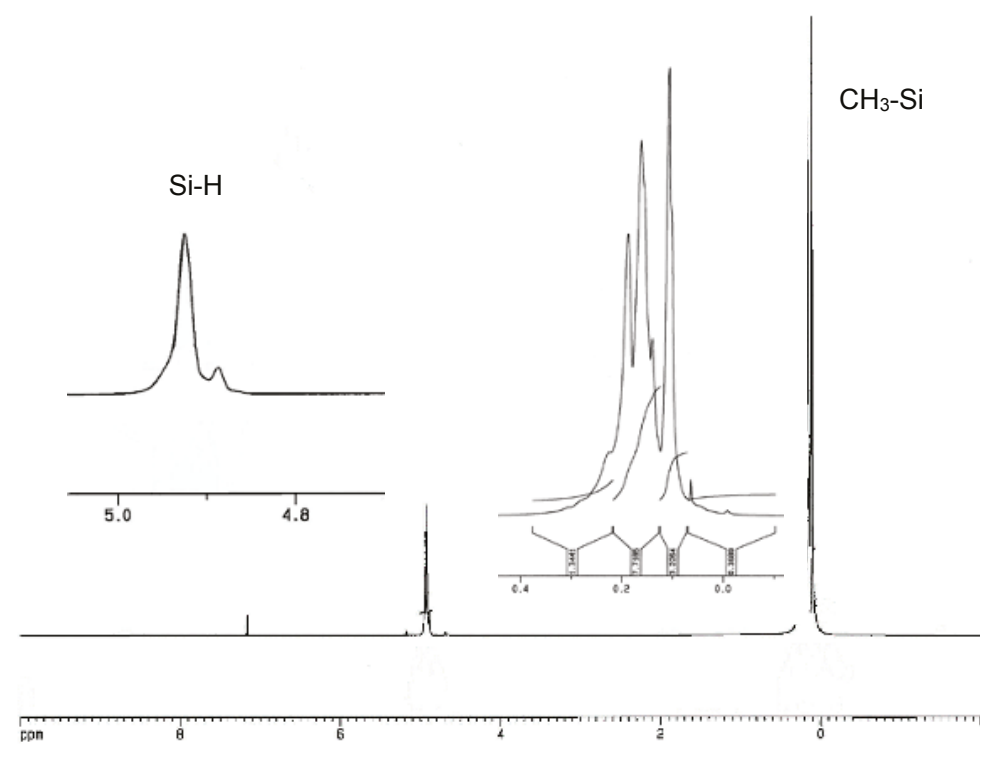

Figure 6. ${ }^{1} \mathrm{H}$-NMR spectrum (in $\mathrm{C}_{6} \mathrm{D}_{6}$ ) of polymethylhydrosiloxane $\mathrm{Q}_{2} \mathrm{D}_{49} \mathrm{D}^{\mathrm{H}}{ }_{49} \mathrm{M}_{6}$. 
Chemical shifts at $\delta$ 9-11 ppm have been assigned to resonances of $\mathrm{Si}$ atoms of $\mathrm{Me}_{3} \mathrm{SiO}_{0.5}(\mathrm{M})$ groups in the following sequences of the siloxane chain ends: $\underline{M} D^{H} D^{H} D^{H}, \underline{M} D^{H} D^{H} D, \underline{M} D^{H} D D^{H}$, $\underline{\mathrm{M}} \mathrm{D}^{\mathrm{H}} \mathrm{DD}, \underline{\mathrm{M}} \mathrm{D}^{\mathrm{H}} \mathrm{D}^{\mathrm{H}} \mathrm{Q}, \underline{\mathrm{M}} \mathrm{D}^{\mathrm{H}} \mathrm{QD}{ }^{\mathrm{H}}, \underline{\mathrm{M}} \mathrm{D}^{\mathrm{H}} \mathrm{DQ}$, and $\underline{\mathrm{M}} \mathrm{D}^{\mathrm{H}} \mathrm{QD}$, while chemical shifts at $\delta 7-8$ ppm belong to resonances of $\mathrm{Si}$ atoms of end groups $\mathrm{M}$ in the sequences: $\underline{\mathrm{MDDD}}, \underline{\mathrm{MDDD}}{ }^{\mathrm{H}}, \underline{\mathrm{MDD}}{ }^{\mathrm{H}} \mathrm{D}, \underline{\mathrm{M} D D^{\mathrm{H}}} \mathrm{D}^{\mathrm{H}}$, $\underline{\mathrm{M} D D Q}, \underline{\mathrm{M} D Q D}, \underline{\mathrm{MQDD}}$, and $\underline{\mathrm{M} Q D D^{\mathrm{H}}}$. Chemical shifts of middle silicon atoms of units $\mathrm{D}$ change in pentades, and magnetic interactions are shifted through four bonds in chain ends.

The sequences of dimethylsiloxane linkages in polymethylhydrosiloxane copolymers might be the following: $\mathrm{DD}^{\mathrm{H}} \underline{\mathbf{D}} \mathrm{D}^{\mathrm{H}} \mathrm{D}, \mathrm{DD}^{\mathrm{H}} \underline{\mathbf{D}} \mathrm{D}^{\mathrm{H}} \mathrm{D}^{\mathrm{H}}, \mathrm{D}^{\mathrm{H}} \mathrm{D}^{\mathrm{H}} \underline{\mathbf{D}} \mathrm{D}^{\mathrm{H}} \mathrm{D}^{\mathrm{H}}, \mathrm{QD}^{\mathrm{H}} \underline{\mathbf{D}} \mathrm{D}^{\mathrm{H}} \mathrm{Q}, \mathrm{MD}^{\mathrm{H}} \underline{\mathbf{D}} \mathrm{D}^{\mathrm{H}} \mathrm{D}, \mathrm{MD}^{\mathrm{H}} \underline{\mathbf{D}} \mathrm{D}^{\mathrm{H}} \mathrm{D}^{\mathrm{H}}$, $M D^{\mathrm{H}} \underline{\mathbf{D}} \mathrm{D}^{\mathrm{H}} \mathrm{Q}, \mathrm{MD}^{\mathrm{H}} \underline{\mathbf{D}} \mathrm{D}^{\mathrm{H}} \mathrm{D}(\delta$ of middle silicon atoms of units $\underline{\mathbf{D}}$ : $-20--22 \mathrm{ppm})$, and: $\mathrm{D}^{\mathrm{H}} \mathrm{D} \underline{\mathbf{D}} \mathrm{D}^{\mathrm{H}} \mathrm{D}^{\mathrm{H}}$, $\mathrm{D}^{\mathrm{H}} \underline{\mathrm{D}} \mathrm{D}^{\mathrm{H}} \mathrm{D}, \mathrm{DD} \underline{\mathbf{D}} \mathrm{D}^{\mathrm{H}} \mathrm{D}^{\mathrm{H}}, \mathrm{DD} \underline{\mathbf{D}} \mathrm{D}^{\mathrm{H}} \mathrm{D}(\delta$ of middle silicon atoms of units $\underline{\mathbf{D}}$ : -18--19.7 ppm).

In the ${ }^{29} \mathrm{Si}-\mathrm{NMR}$ spectra (recorded by INEPT and INVGATE techniques) in the range of $\delta$ $-33--37$ ppm exist signals of middle silicon atoms of units $\mathbf{D}^{\mathrm{H}}$, which undergo changes in pentades (Table 5). Signals of silicon atoms in the range of $\delta-102$ to $-109 \mathrm{ppm}$, presumably correspond to $\mathrm{Si}$ atoms in the central units $\underline{\mathbf{Q}}$, in the following sequences of siloxane structures:<smiles>[2H][Si]([2H])([2H])[2H]</smiles><smiles>[R][R]([2H])([2H])CC</smiles><smiles>[2H][13C]([2H])([2H])[2H]</smiles><smiles>[R][R]([2H])([2H])C</smiles><smiles>[R][Si]([2H])([2H])O</smiles>

Chemical shifts in the range of $7-11 \mathrm{ppm}$ in the ${ }^{29} \mathrm{Si}-\mathrm{NMR}$ spectra (INEPT and INVGATE) correspond to silicon atoms of the end groups $\mathrm{M}$ and change in tetrads (Table 5) $[8,9,74]$.

Signals at $\delta-64 \mathrm{ppm}$ of a very low intensity, registered both in INVGATE and INEPT ${ }^{29} \mathrm{Si}-\mathrm{NMR}$ spectra of these three copolymers, probably come from $\mathrm{Si}$ atoms of units $\mathrm{MeSiO}_{1.5}(\mathrm{~T})$, which were formed during syntheses of PMHS-Q from trace hydrolysis of Si-H bonds [74].

Assignments of all ${ }^{29} \mathrm{Si}-\mathrm{NMR}$ signals resulting from the microstructure of siloxane chain of branched polymethylhydrosiloxanes are summarized in Table 6 . 


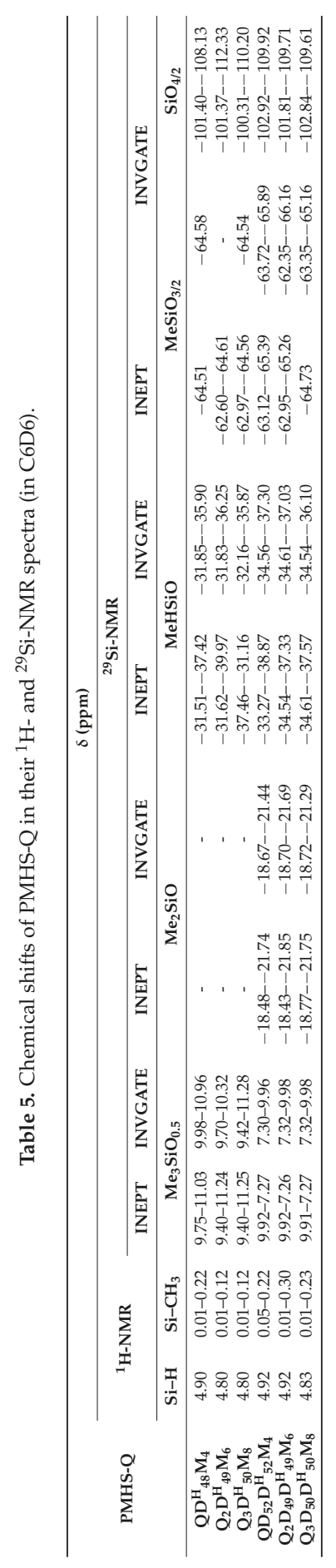




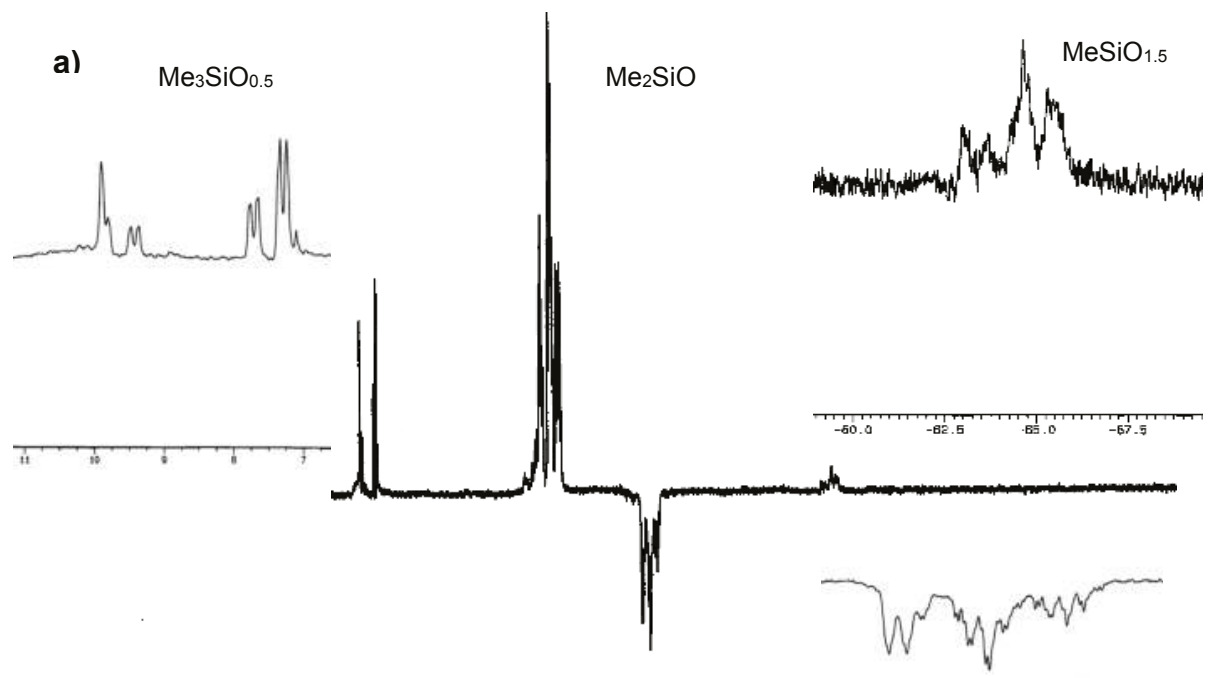

$\mathrm{MeHSiO}$

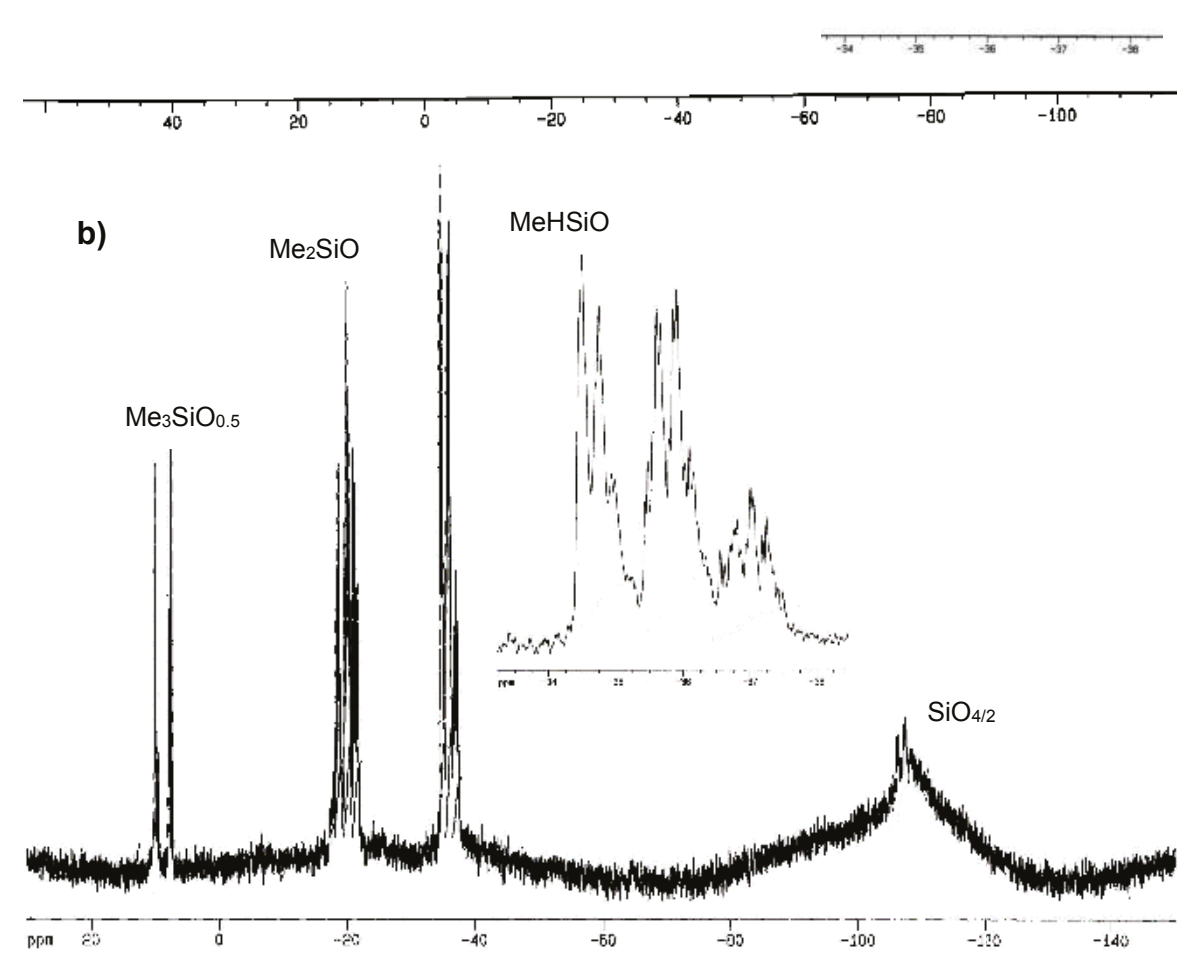

Figure 7. ${ }^{29} \mathrm{Si}-\mathrm{NMR}$ spectra (in $\mathrm{C}_{6} \mathrm{D}_{6}$ ) of polymethylhydrosiloxane $\mathrm{Q}_{2} \mathrm{D}_{49} \mathrm{D}^{\mathrm{H}}{ }_{49} \mathrm{M}_{6}$ : (a) INEPT, (b) INVGATE. 
Table 6. The microstructure of siloxane chains in PMHS-Q, containing quadruple branching units Q (all possible sequences among tetrads of terminal groups, and linear and star pentads); values of $\delta$ concern Si atoms in structural units denoted as bold and underlined.

\begin{tabular}{|c|c|c|c|c|c|}
\hline \multicolumn{6}{|c|}{$\delta^{29} \mathrm{Si}-\mathrm{NMR}(\mathrm{ppm})$} \\
\hline 9-11 & $7-8$ & $-18--19$ & $-20--22$ & $-33--37$ & $-101--109$ \\
\hline \multirow[t]{2}{*}{$\underline{\mathbf{M}} \mathrm{D}^{\mathrm{H}} \mathrm{D}^{\mathrm{H}} \mathrm{D}^{\mathrm{H}}$} & MDDD & $\mathrm{DD}^{\mathrm{H}} \underline{\mathbf{D}} \mathrm{D}^{\mathrm{H}} \mathrm{D}$ & $\mathrm{D}^{\mathrm{H}} \mathrm{D} \underline{\mathrm{D}} \mathrm{D}^{\mathrm{H}} \mathrm{D}^{\mathrm{H}}$ & $\mathrm{D}^{\mathrm{H}} \mathrm{D}^{\mathrm{H}} \underline{\mathbf{D}}^{\mathrm{H}} \mathrm{D}^{\mathrm{H}} \mathrm{D}^{\mathrm{H}}$ & \\
\hline & & & $\mathrm{D}^{\mathrm{H}} \mathrm{DD} \mathrm{D}^{\mathrm{H}} \mathrm{D}$ & $\mathrm{DD}^{\mathrm{H}} \mathbf{D}^{\mathrm{H}} \mathrm{D}^{\mathrm{H}} \mathrm{D}^{\mathrm{H}}$ & $\mathrm{D}$ \\
\hline \multirow[t]{2}{*}{$\underline{\mathbf{M}} \mathrm{D}^{\mathrm{H}} \mathrm{D}^{\mathrm{H}} \mathrm{Q}$} & $\underline{\mathbf{M D D D}^{\mathrm{H}}}$ & $\mathrm{DD}^{\mathrm{H}} \underline{\mathbf{D}} \mathrm{D}^{\mathrm{H}} \mathrm{D}^{\mathrm{H}}$ & $\mathrm{DDDD}{ }^{\mathrm{H}} \mathrm{D}^{\mathrm{H}}$ & $\mathrm{D}^{\mathrm{H}} \mathrm{D} \underline{\mathrm{H}}^{\mathrm{H}} \mathrm{D}^{\mathrm{H}} \mathrm{D}^{\mathrm{H}}$ & DQD \\
\hline & & & $\mathrm{DDD} \mathrm{D}^{\mathrm{H}} \mathrm{D}$ & $\mathrm{DD}^{\mathrm{H}} \underline{\mathrm{D}}^{\mathrm{H}} \mathrm{D}^{\mathrm{H}} \mathrm{D}$ & $\widehat{\bar{D}}$ \\
\hline \multirow[t]{2}{*}{$\underline{\mathbf{M}}{ }^{\mathrm{H}} \mathrm{QD}^{\mathrm{H}}$} & $\underline{\mathbf{M D D}}{ }^{\mathrm{H}} \mathrm{D}$ & $\mathrm{D}^{\mathrm{H}} \mathrm{D}^{\mathrm{H}} \underline{\mathbf{D}} \mathrm{D}^{\mathrm{H}} \mathrm{D}^{\mathrm{H}}$ & $\mathrm{D}^{\mathrm{H}} \mathrm{DDDQ}$ & $\mathrm{DD}^{\mathrm{H}} \underline{\mathbf{D}}^{\mathrm{H}} \mathrm{DD}^{\mathrm{H}}$ & \\
\hline & & & $\mathrm{D}^{\mathrm{H}} \mathrm{DDQD}$ & $\mathrm{D}^{\mathrm{H}} \mathrm{D} \mathrm{D}^{\mathrm{H}} \mathrm{D} \mathrm{D}^{\mathrm{H}}$ & \\
\hline \multirow[t]{2}{*}{$\underline{\mathbf{M}} \mathrm{D}^{\mathrm{H}} \mathrm{D}^{\mathrm{H}} \mathrm{D}$} & $\underline{\mathbf{M}} D^{\mathrm{H}} \mathrm{D}^{\mathrm{H}}$ & $\mathrm{DD}^{\mathrm{H}} \underline{\mathbf{D}} \mathrm{D}^{\mathrm{H}} \mathrm{Q}$ & $\mathrm{DD}^{\mathrm{H}} \underline{\mathrm{D}} \mathrm{DQ}$ & $\mathrm{D}^{\mathrm{H}} \mathrm{DD}^{\mathrm{H}} \mathrm{D}^{\mathrm{H}} \mathrm{Q}$ & $\mathrm{D}$ \\
\hline & & & $\mathrm{DD}^{\mathrm{H}} \underline{\mathrm{DQD}}$ & $\mathrm{D}^{\mathrm{H}} \mathrm{D}^{\mathrm{H}} \underline{\mathbf{D}}^{\mathrm{H}} \mathrm{QD}^{\mathrm{H}}$ & DQQD \\
\hline \multirow[t]{2}{*}{$\underline{\mathbf{M}} \mathrm{D}^{\mathrm{H}} \mathrm{D}^{\mathrm{H}} \mathrm{D}^{\mathrm{H}}$} & MDDQ & $\mathrm{QD}^{\mathrm{H}} \underline{\mathbf{D}} \mathrm{D}^{\mathrm{H}} \mathrm{Q}$ & DDDDQ & $\mathrm{DD}^{\mathrm{H}} \underline{\mathbf{D}}^{\mathrm{H}_{\mathrm{H}}} \mathrm{D}^{\mathrm{H}} \mathrm{Q}$ & $\overline{\mathrm{M}}$ \\
\hline & & & DDD̄QD & $\mathrm{D}^{\mathrm{H}} \mathrm{DD}^{\mathrm{H}} \mathrm{D}^{\mathrm{H}} \mathrm{Q}$ & \\
\hline \multirow[t]{2}{*}{$\underline{\mathbf{M}} \mathrm{D}^{\mathrm{H}} \mathrm{DD}^{\mathrm{H}}$} & $\underline{\text { MDQD }}$ & $\mathrm{MD}^{\mathrm{H}} \underline{\mathbf{D}} \mathrm{D}^{\mathrm{H}} \mathrm{D}$ & QDDDQ & $\mathrm{D}^{\mathrm{H}} \mathrm{DD}^{\mathrm{H}} \mathrm{QD}^{\mathrm{H}}$ & \\
\hline & & & QDD̄QDD & $\mathrm{DD}^{\mathrm{H}} \mathbf{D}^{\mathrm{HH}} \mathrm{QD}^{\mathrm{H}}$ & $\mathrm{D}$ \\
\hline \multirow[t]{22}{*}{$\underline{\mathbf{M}} \mathrm{D}^{\mathrm{H}} \mathrm{DD}$} & $\underline{\text { MQDD }}$ & $\mathrm{MD}^{\mathrm{H}} \underline{\mathbf{D}} \mathrm{D}^{\mathrm{H}} \mathrm{D}^{\mathrm{H}}$ & DQDQD & $\mathrm{DDD}^{\mathrm{H}} \mathrm{QD}^{\mathrm{H}}$ & DQM \\
\hline & & & DQDDQ & $\mathrm{D}^{\mathrm{H}} \mathrm{D}^{\mathrm{H}} \mathrm{D}^{\mathrm{H}} \mathrm{DQ}$ & $\overline{\mathrm{M}}$ \\
\hline & $\underline{\mathbf{M Q D D}}{ }^{\mathrm{H}}$ & $\mathrm{MD}^{\mathrm{H}} \underline{\mathbf{D}} \mathrm{D}^{\mathrm{H}} \mathrm{Q}$ & MDDDDQ & $\mathrm{D}^{\mathrm{H}} \mathrm{D}^{\mathrm{H}} \underline{\mathbf{D}}^{\mathrm{H}} \mathrm{QD}$ & \\
\hline & & & MDDQD & $\mathrm{DD}^{\mathrm{H}} \underline{\mathbf{D}}^{\mathrm{H}}$ & \\
\hline & & $\mathrm{MD}^{\mathrm{H}} \underline{\mathbf{D}} \mathrm{D}^{\mathrm{H}} \mathrm{D}$ & MQDDD & $\mathrm{DD}^{\mathrm{H}} \underline{\mathbf{D}}^{\mathbf{H}} \mathrm{QD}$ & $\mathrm{D}$ \\
\hline & & & MDDDQ & $\mathrm{D}^{\mathrm{H}} \mathrm{DD}^{\mathrm{H}} \mathrm{DQ}$ & DQQD \\
\hline & & & MQDQD & $\mathrm{D}^{\mathrm{H}} \mathrm{DD}^{\mathrm{H}} \mathrm{QD}$ & $\mathrm{D}$ \\
\hline & & & & $\mathrm{DDD}^{\mathrm{H}} \mathrm{D}^{\mathrm{H}} \mathrm{Q}$ & \\
\hline & & & & $\mathrm{DDD}^{\mathrm{H}} \mathrm{DQ}$ & D \\
\hline & & & & $\mathrm{MD}^{\mathrm{H}} \overline{\mathbf{D}^{\mathrm{H}}} \mathrm{D}^{\mathrm{H}} \mathrm{D}^{\mathrm{H}}$ & D \\
\hline & & & & $\mathrm{MD}^{\mathrm{H}} \underline{\overline{\mathbf{D}^{\mathrm{H}}}} \mathrm{D}^{\mathrm{H}} \mathrm{D}$ & $\mathrm{DQD}^{\mathrm{H}}$ \\
\hline & & & & $\mathrm{MD}^{\mathrm{H}} \underline{\mathrm{D}}^{\mathrm{H}} \mathrm{DD}^{\mathrm{H}}$ & \\
\hline & & & & $M D D^{\mathrm{H}} \mathrm{D}^{\mathrm{H}} \mathrm{D}^{\mathrm{H}}$ & \\
\hline & & & & $\mathrm{MDD}^{\mathrm{H}} \mathrm{D}^{\mathrm{H}} \mathrm{D}$ & \\
\hline & & & & $\mathrm{MD} \overline{\mathrm{D}^{\mathrm{H}}} \mathrm{DD}^{\mathrm{H}}$ & $\begin{array}{c}M \\
D O D\end{array}$ \\
\hline & & & & $M D^{\overline{\mathrm{H}}} \underline{\mathbf{D}}^{\mathrm{H}} \mathrm{D}^{\mathrm{H}} \mathrm{Q}$ & $\frac{\mathrm{DQ}}{\mathrm{D}^{\mathrm{H}}}$ \\
\hline & & & & $\mathrm{MDD} \overline{\mathrm{D}^{\mathrm{H}}} \mathrm{D}^{\mathrm{H}} \mathrm{Q}$ & \\
\hline & & & & $M D^{\overline{\mathrm{H}}} \underline{\mathbf{D}}^{\mathrm{H}} \mathrm{DQ}$ & \\
\hline & & & & $\mathrm{MD}^{\mathrm{H}} \underline{\mathrm{D}^{\mathrm{H}}} \mathrm{QD}$ & \\
\hline & & & & $\mathrm{MD}^{\mathrm{H}} \mathbf{D}^{\mathrm{H}} \mathrm{QD}^{\mathrm{H}}$ & \\
\hline & & & & $\mathrm{MDD}^{\mathrm{H}} \mathrm{QD}^{\mathrm{H}}$ & \\
\hline & & & & $\mathrm{MDD^{H }} D Q$ & \\
\hline
\end{tabular}

Author Contributions: Syntheses and characterization of branched PMHS were carried out by J.J. Chruściel and M. Fejdyś. Moreover, J.J. Chruściel measured dynamic viscosities of PMHS, M. Fejdyś (35\% total contribution) recorded FT-IR spectra, and W. Fortuniak (10\% contribution) performed measurements of molecular weights of PMHS by the SEC method. A manuscript has been written by J.J. Chruściel (55\% total contribution).

Acknowledgments: The authors would like to acknowledge the Ministry of Science and Higher Education (Poland), for a financial support through a grant 3 T08E 053 30. We are also very grateful to a Management of the Institute of Polymers and Dye Technology, Lodz University of Technology, and especially to Director Professor Marian Zaborski, for providing laboratories.

Conflicts of Interest: The authors declare no conflict of interest.

\section{References}

1. Eaborn, C. Organosilicon Compounds; Butterworth Scientific Publications: London, UK, 1960.

2. Noll, W. Chemie und Technologie der Silikone; 2 Auflage; Verlag Chemie: Weinheim Germany; GmbH: Weinheim Germany, 1968. 
3. Hardman, B.; Torkelson, A. Chapter Silicones. In Encyclopedia of Polymer Science and Engineering, 2nd ed.; Mark, F., Gaylord, N.G., Bikales, N.M., Eds.; J. Wiley \& Sons: New York, NY, USA, 1989; Volume 15, pp. 271-289.

4. Rościszewski, P.; Zielecka, M. Silikony, Właściwości i Zastosowanie; WNT: Warsaw, Poland, 2002; pp. 26-30, 38.

5. Chauhan, B.P.S. (Ed.) Hybrid Nanomaterials Synthesis, Characterization, and Applications; Wiley-VCH: Weinheim, Germany, 2011.

6. Kricheldorf, H.R. (Ed.) Silicon in Polymer Synthesis; Springer: Berlin/Heidelberg, Germany; New York, NY, USA, 1996.

7. Brook, M.A. Silicon in Organic, Organometallic and Polymer Chemistry; J Wiley \& Sons: New York, NY, USA; Chichester, UK; Weinheim, Germany; Brisbane, Australia; Singapore; Toronto, Japan, 2000; ISBN 0-471-19658-4.

8. Chruściel, J. Progress in chemistry of polymethylhydrosiloxanes. Polimery 1999, 44, 462-474.

9. Chruściel, J. Synthesis and characterization of new liquid, branched and random poly(methyl-hydrosiloxanes). e-Polymers 2008, 8, 1-23. [CrossRef]

10. Chruściel, J. Crosslinking of polydimethylsiloxane- $\alpha, \omega$-diols with polymethylhydrosiloxanes and some properties of prepared silicone elastomers. Polimery 1999, 44, 586-596.

11. Devimille, L.; Bresson, B.; Bokobza, L. Synthesis, structure and morphology of poly(dimethyl-siloxane) networks filled with in situ generated silica particles. Polymer 2005, 46, 4135-4143. [CrossRef]

12. Fejdyś, M.; Chruściel, J.; Miazga, A. Mechanical properties of silicone vulcanizates crosslinked with polymethylhydrosiloxanes containing silica and calcium carbonate. Polimery 2006, 51, 48-57.

13. Jia, L.; Du, Z.; Zhang, C.; Liu, C.; Li, H. Reinforcement of polydimethylsiloxane through formation of inorganic-organic hybrid network. Polym. Eng. Sci. 2008, 48, 74-79. [CrossRef]

14. Urayama, K.; Kawamura, T.; Kohjiya, S. Structure-mechanical property correlations of model siloxane elastomers with controlled network topology. Polymer 2009, 50, 347-356. [CrossRef]

15. Chruściel, J.; Leśniak, E. Preparation of flexible, self-extinguishing silicone foams. J. Appl. Polym. Sci. 2011, 119, 1696-1703. [CrossRef]

16. Hager, R. Phosphazene catalysis-Basic technology in silicone production. In Proceedings of the XI International Symposium on Organosilicon Chemistry, Montpellier, France, 1-6 September 1996; Universite Montpellier II: Montpellier, France, 1996. Abstracts, LD5.

17. Hager, R.; Schneider, O.; Schuster, J. Process for the Condensation and/or Equilibration of Organosilicon Compounds. U.S. Patent 5,380,902, 1995.

18. Razzano, J.S.; Anderson, P.P.; Perry, R.J. Production of Low Molecular Weight Linear Hydrogen Siloxanes. U.S. Patent 5,670,596, 23 September 1997.

19. Nye, S.A.; Riccio, D.A.; Wutzer, B.S. Process for Preparing Hydrogen Siloxane Copolymers. U.S. Patent 5,698,654, 1997.

20. Liao, W.P.; Nye, S.A. Process for Preparing Self-Curable Alkenyl Hydride Siloxane Copolymers and Coating Composition. European Patent EP 0838547 B1, 1998.

21. Palaprat, G.; Ganachaud, F.; Mauzac, M.; Hemery, P. Cationic polymerization of 2,4,6,8-tetramethylcyclotetrasiloxane processed by tuning the $\mathrm{pH}$ of the miniemulsion. Polymer 2005, 46, 11213-11218. [CrossRef]

22. Maisonnier, S.; Favier, J.C.; Masure, M.; Hemery, P. Poly(methylhydrosiloxane) synthesis in anionic aqueous emulsion. In Proceedings of the XI International Symposium on Organosilicon Chemistry, Montpellier, France, 1-6 September 1996; Universite Montpellier II: Montpellier, France, 1996. Abstracts, PB84.

23. Paulasaari, J.K.; Weber, W.P. Preparation of Highly Regular Poly(1-hydrido-1,3,3,5,5- -pentamethyltrisiloxane) and Its Chemical Modification by Hydrosilylation. Macromolecules 1999, 32, 6574-6577. [CrossRef]

24. Agaskar, P.A. Facile, High-Yield Synthesis of Functionalized Spherosilicates: Precursors of Novel Organolithic Macromolecular Materials. Inorg. Chem. 1990, 29, 1603. [CrossRef]

25. Chruściel, J.; Kulpiński, J.; Romanowski, Z. Synthesis of Some Block Polymethylhydrosiloxanes. Zeszyty Naukowe Politechniki Śląskiej Seria Chemia 1999, 140, 109-114.

26. Agaskar, P.A. New Synthetic Route to the Hydridospherosiloxanes $\mathrm{O}_{h}-\mathrm{HSi}_{8} \mathrm{O}_{12}$ and $D_{5 h}-\mathrm{H}_{10} \mathrm{Si}_{10} \mathrm{O}_{15}$. Inorg. Chem. 1991, 30, 2707-2708. [CrossRef]

27. Bassindale, A.R.; Gentle, T.E. Siloxane and Hydrocarbon Octopus Molecules with Silsesquioxane Cores. J. Mater. Chem. 1993, 3, 1319-1325. [CrossRef] 
28. Crivello, J.V.; Malik, R. Synthesis and Photoinitiated Cationic Polymerization of Monomers with the Silsesquioxane Core. J. Polym. Sci. Part A Polym. Chem. 1997, 35, 407-425. [CrossRef]

29. Tsuchida, A.; Bolin, C.; Sernetz, F.G.; Frey, H.; Mülhaupt, R. Ethene and Propene Copolymers Containing Silsesquioxane Side Groups. Macromolecules 1997, 30, 2818-2824. [CrossRef]

30. Zhang, C.; Babonneau, F.; Laine, R.M.; Soles, C.L.; Hristov, H.A.; Yee, A.F. Highly Porous Polyhedral Silsesquioxane Polymers. Synthesis and Characterization. J. Am. Chem. Soc. 1998, 120, 8380-8391. [CrossRef]

31. Zhang, C.; Laine, R.M. Hydrosilylation of Allyl Alcohol with $\left[\mathrm{HSiMe}_{2} \mathrm{OSiO}_{1.5}\right]_{8}$ : Octa(3-hydroxypropyldimethylsiloxy)octasilsesquioxane and Its Octamethacrylate Derivative as Potential Precursors to Hybrid Nanocomposites. J. Am. Chem. Soc. 2000, 122, 6979-6988. [CrossRef]

32. Constantopoulos, K.; Clarke, D.; Markovic, E.; Uhrig, D.; Clarke, S.; Matisons, J.G.; Simon, G. A new family of POSS monomers suitable for forming urethane polymerizable nanocomposite coatings. Polym. Prepr. 2004, 45, 668 .

33. Frye, C.; Collins, W.T. The Oligomeric Silsesquioxanes, $\left(\mathrm{HSiO}_{3 / 2}\right)_{\mathrm{n}}$. J. Am. Chem. Soc. 1970, 92, 5586-5588. [CrossRef]

34. Agaskar, P.A.; Day, V.W.; Klemperer, W.G. A New Route to Trimnethylsilylated Spherosilicates: Synthesis and Structure of $\left[\mathrm{Si}_{12} \mathrm{O}_{18}\right]\left(\mathrm{OSiMe}_{3}\right)_{12}, D_{3 h}-\left[\mathrm{Si}_{14} \mathrm{O}_{21}\right]\left(\mathrm{OSiMe}_{3}\right)_{14}$, and $C_{2 v}-\left[\mathrm{Si}_{14} \mathrm{O}_{21}\right]\left(\mathrm{OSiMe}_{3}\right)_{14}$. J. Am. Chem. Soc. 1987, 109, 5554-5556. [CrossRef]

35. Hasegawa, I.; Motojima, D. Dimethylvinylsilylation of $\mathrm{Si}_{8} \mathrm{O}_{20}{ }^{8-}$ silicate anion in methanol solutions of tetramethylammonium silicate. J. Organomet. Chem. 1992, 441, 373-380. [CrossRef]

36. Majoros, I.; Marsylko, T.M.; Kennedy, J.P. Synthesis and characterization of novel well-defined stars consisting of eight polyisobutylene arms emanating from an octa(dimethylsiloxy)octasilsesquioxane core. Polym. Bull. 1997, 38, 15-22. [CrossRef]

37. Provatas, A.; Luft, M.; Mu, J.C.; White, A.H.; Matisons, J.G.; Skelton, B.W. Silsesquioxanes: Part I: A key intermediate in the building of molecular composite materials. J. Organomet. Chem. 1998, 565, 159-164. [CrossRef]

38. Perrin, F.X.; Nguyen, T.B.V.; Margaillan, A. Linear and branched alkyl substituted octakis(dimethylsiloxy)octasilsesquioxanes: WAXS and thermal properties. Eur. Polym. J. 2011, 47, 1370-1382. [CrossRef]

39. Dutkiewicz, M.; Maciejewski, H.; Marciniec, B.; Karasiewicz, J. New Fluorocarbofunctional Spherosilicates: Synthesis and Characterization. Organometallics 2011, 30, 2149-2153. [CrossRef]

40. Hasegawa, I. Co-hydrolysis products of tetraethoxysilane and methyltriethoxysilane in the presence of tetramethylammonium ions. J. Sol-Gel Sci. Technol. 1993, 1, 57-63. [CrossRef]

41. Moran, M.; Casado, C.M.; Cuadrado, I.; Losada, J. Ferrocenyl Substituted Octakis(dimethylsiloxy)octasilsesquioxanes: A New Class of Supramolecular Organometallic Compounds. Synthesis, Characterization, and Electrochemistry. Organometallics 1993, 12, 4327-4333. [CrossRef]

42. Sellinger, A.; Laine, R.M. Silsesquioxanes as Synthetic Platforms. 3. Photocurable, Liquid Epoxides as Inorganic/Organic Hybrid Precursors. Chem. Mater. 1996, 8, 1592-1593. [CrossRef]

43. Laine, R.M. Nanobuilding blocks based on the $\left[\mathrm{OSiO}_{1.5}\right]_{\mathrm{x}}(\mathrm{x}=6,8,10)$ Octasilsesquioxanes. J. Mater. Chem. 2005, 15, 3725-3744. [CrossRef]

44. Hasegawa, I.; Ino, K.; Onishi, H. An improved procedure for syntheses of silyl derivatives of the cubeoctameric silicate anion. Appl. Organomet. Chem. 2003, 17, 287-290. [CrossRef]

45. Soh, M.S.; Yap, A.U.J.; Sellinger, A. Methacrylate and epoxy functionalized nanocomposites based on silsesquioxane cores for use in dental applications. Eur. Polym. J. 2007, 43, 315-327. [CrossRef]

46. Hessel, C.M.; Henderson, E.J.; Veinot, J.G.C. Hydrogen Silsesquioxane: A Molecular Precursor for Nanocrystalline $\mathrm{Si}_{-} \mathrm{SiO}_{2}$ Composites and Freestanding Hydride-Surface-Terminated Silicon Nano-particles. Chem. Mater. 2006, 18, 6139-6146. [CrossRef]

47. Gunji, T.; Shioda, T.; Tsuchihira, K.; Seki, H.; Kajiwara, T.; Abe, Y. Preparation and properties of polyhedral oligomeric silsesquioxane-polysiloxane copolymers. Appl. Organomet. Chem. 2010, 24, 545-550. [CrossRef]

48. Shioda, T.; Gunji, T.; Abe, N.; Abe, Y. Preparation and properties of polyhedral oligomeric silsesquioxane polymers. Appl. Organomet. Chem. 2011, 25, 661-664. [CrossRef]

49. Handke, M.; Kowalewska, A. Siloxane and silsesquioxane molecules-Precursors for silicate materials. Spectrochim. Acta Part A 2011, 79, 749-757. [CrossRef] [PubMed] 
50. Cai, G.; Weber, W.P. Synthesis of terminal Si-H irregular tetra-branched star polysiloxanes. Pt-catalyzed hydrosilylation with unsaturated epoxides. Polysiloxane films by photo-acid catalyzed crosslinking. Polymer 2004, 45, 2941-2948. [CrossRef]

51. Grunlan, M.A.; Lee, N.S.; Mansfeld, F.; Kus, E.; Finlay, J.A.; Callow, J.A.; Callow, M.E.; Weber, W.P. Minimally Adhesive Polymer Surfaces Prepared from Star Oligosiloxanes and Star Oligofluorosiloxanes. J. Polym. Sci. Part A Polym. Chem. 2006, 44, 2551-2566. [CrossRef]

52. Sargent, J.R.; Weber, W.P. Telechelic 4 and 6 branch star siloxanes by acid catalyzed incorporation of D4 units into the Si-O-Si bonds of tetra(dimethylsiloxy)silane and hexa(dimethylsiloxy)-cyclotrisiloxane. Polym. Prepr. 2000, 41, 604

53. Cai, G.; Sargent, J.R.; Weber, W.P. Preparation and reactivity of polyfunctional six- and eight-membered cyclic silicates. J. Organomet. Chem. 2004, 689, 689-693. [CrossRef]

54. Saxena, K.; Bisaria, C.S.; Saxena, A.K. Studies on the synthesis and thermal properties of alkoxysilane-terminated organosilicone dendrimers. Appl. Organomet. Chem. 2010, 24, 251-256. [CrossRef]

55. Zhang, R.; Dai, D.; Cui, L.; Xu, H.; Liu, C.; Xie, P. A glance at the relation of stepwise coupling polymerization to supramolecular chemistry. Mater. Sci. Eng. C 1999, 10, 13-18. [CrossRef]

56. Wan, Y.; Xu, L.; Ren, L.; Zhang, L.; Xie, P.; Liu, Y.; Zhang, R. Synthesis and mesomorphic properties of fishbone-like liquid crystalline polysilsesquioxanes V. Pd-coordinating, fishbone-like azo-based liquid crystalline polysilsesquioxanes. Liquid Cryst. 1998, 24, 871-876. [CrossRef]

57. Cao, M.; Li, Z.; Zhang, Y.; Xie, P.; Dai, D.; Zhang, R.; Lin, Y.; Chung, N.T. Synthesis and characterization of ladder-like copolymethyl-epoxysilsesquioxane. React. Funct. Polym. 2000, 45, 119-130. [CrossRef]

58. Uchida, H.; Kabe, Y.; Yoshino, K.; Kawamata, A.; Tsumuraya, T.; Masamune, S. General Strategy for the Systematic Synthesis of Oligosiloxanes. Silicone Dendrimers. J. Am. Chem. Soc. 1990, 112, 7077-7079. [CrossRef]

59. Zhao, S.; Feng, S. Hydrogen-Containing Silicone Resin as the Crosslinking Agent of Heat-Curable Silicone Rubber. J. Appl. Polym. Sci. 2003, 88, 3066-3069. [CrossRef]

60. Su, H.W.; Chen, W.C.; Lee, W.C.; King, J.S. New Photocurable Acrylic/Silsesquioxane Hybrid Optical Materials: Synthesis, Properties, and Patterning. Macromol. Mater. Eng. 2007, 292, 666-673. [CrossRef]

61. Seino, M.; Hayakawa, T.; Ishida, Y.; Kakimoto, M. Hydrosilylation Polymerization of Double-Decker-Shaped Silsesquioxane Having Hydrosilane with Diynes. Macromolecules 2006, 39, 3473-3475. [CrossRef]

62. Soraru, G.D.; D'Andrea, G.; Campostrini, R.; Baboneau, F. Characterization of Methyl-substituted Silica Gels with Si-H Functionalities. J. Mater. Chem. 1995, 5, 1363-1374. [CrossRef]

63. Campostrini, R.; D’Andrea, G.; Carturan, G.; Ceccato, R.; Soraru, G.D. Pyrolysis study of methyl-substituted $\mathrm{Si}-\mathrm{H}$ containing gels as precursors for oxycarbide glasses, by combined thermo-gravimetry, gas chromatographic and mass spectrometric analysis. J. Mater. Chem. 1996, 6, 585-594. [CrossRef]

64. Bažant, V.; Chvalovský, V.; Rathouský, I. Silikony; PWT: Warsaw, Poland, 1955; p. 153.

65. Morikawa, A.; Kakimoto, M.; Imai, Y. Synthesis and Characterization of New Polysiloxane Starburst Polymers. Macromolecules 1991, 24, 3469-3474. [CrossRef]

66. Chang, H.T.; Frechet, J.M.J. Proton-Transfer Polymerization: A New Approach to Hyperbranched Polymers. J. Am. Chem. Soc. 1999, 121, 2313-2314. [CrossRef]

67. Innoue, K. Functional dendrimers, hyperbranched and star polymers. Prog. Polym. Sci. 2000, 25, 453-571. [CrossRef]

68. Paulasaari, J.K.; Weber, W.P. Synthesis of Hyperbranched Polysiloxanes by Base-Catalyzed Proton-Transfer Polymerization. Comparison of Hyperbranched Polymer Microstructure and Properties to Those of Linear Analogues Prepared by Cationic or Anionic Ring-Opening Polymerization. Macromolecules 2000, 33, 2005-2010. [CrossRef]

69. Paulasaari, J.K.; Weber, W.P. Base catalyzed proton transfer polymerization of 1-hydroxypentamethylcyclotrisiloxane. Comparison of hyperbranched polymer microstructure and properties to those of highly regular linear analogs. Macromol. Chem. Phys. 2000, 201, 1585-1592. [CrossRef]

70. Jikei, M.; Kakimoto, M. Hyperbranched polymers: A promising new class of materials. Prog. Polym. Sci. 2001, 26, 1233-1285. [CrossRef]

71. Servaty, S.; Köhler, W.; Meyer, W.H.; Rosenauer, C.; Spickermann, J.; Räder, H.J.; Wegner, G. MALDI-TOF-MS Copolymer Analysis: Characterization of a Poly(dimethylsiloxane)--co-Poly(hydromethylsiloxane) as a Precursor of a Functionalized Silicone Graft Copolymer. Macromolecules 1998, 31, 2468-2474. [CrossRef] 
72. Silverstein, R.M.; Bassler, G.C. Spektroskopowe Metody Identyfikacji Związków Organicznych; PWN: Warsaw, Poland, 1969; pp. 72-118.

73. Glaser, R.H.; Wilkes, G.L.; Bronnimann, C.E. Solid-state ${ }^{29}$ Si NMR of TEOS-based multifunctional sol-gel materials. J. Non-Cryst. Solids 1989, 113, 73-87. [CrossRef]

74. Harris, R.K.; Kimber, B.J. ${ }^{29}$ Si nuclear magnetic resonance studies of some oligo- and polymeric siloxanes. J. Organomet. Chem. 1974, 70, 43-49. [CrossRef]

(C) 2018 by the authors. Licensee MDPI, Basel, Switzerland. This article is an open access article distributed under the terms and conditions of the Creative Commons Attribution (CC BY) license (http:/ / creativecommons.org/licenses/by/4.0/). 
Article

\title{
Hyperbranched Polysiloxanes Based on Polyhedral Oligomeric Silsesquioxane Cages with Ultra-High Molecular Weight and Structural Tuneability
}

\author{
Ning Liu ${ }^{1}$, Jianyi Yu ${ }^{2}$, Yaoyong Meng ${ }^{1, *}$ and Yuzhou Liu ${ }^{2,3, *}$ \\ 1 MOE Key Laboratory of Laser Life Science \& Laboratory of Photonic Chinese Medicine, College of \\ Biophotonics, South China Normal University, Guangdong 510631, China; liuningmengqi@163.com \\ 2 Beijing Advanced Innovation Center for Biomedical Engineering, Beihang University, Beijing 100191, China; \\ yujianyi9058@163.com \\ 3 School of Chemistry, Beihang University, Beijing 100191, China \\ * Correspondence: yaoyongmeng@aliyun.com (Y.M.); liuyuzhou@buaa.edu.cn (Y.L.); \\ Tel.: +86-20-3931-0266 (Y.M.); +86-10-8231-6866 (Y.L.)
}

Received: 14 March 2018; Accepted: 9 April 2018; Published: 5 May 2018

\begin{abstract}
Hyperbranched siloxane-based polymers with ultra-high molecular weight were synthesized by the Piers-Rubinsztajn reaction between octakis(dimethylsiloxy) octasilsesquioxane with different dialkoxysilanes, using tris(pentafluorophenyl) borane as the catalyst. The origin of the high molecular weight is explained by the high reactivity of the catalyst and strain energy of isolated small molecule in which all eight silane groups close into rings on the sides of a single cubic cage. The structural tuneability was further demonstrated by use of methyl(3-chloropropyl)diethoxysilane, which generates a polymer with similar ultra-high molecular weight. Introduction of phosphonate groups through the chloropropyl sites later leads to functionalized polymers which can encapsulate various transition metal nanoparticles.
\end{abstract}

Keywords: polyhedral oligomeric silsesquioxanes; high molecular weight; nanoparticles

\section{Introduction}

Polyhedral oligomeric silsesquioxanes (POSSs), as first synthesized by D.W. Scott in 1946 [1], have attracted considerable attention. POSSs are polysiloxanes consisting of the structure unit of $\left(\mathrm{RSiO}_{3 / 2}\right)_{\mathrm{n}}$, and they can exist in different forms, including amorphous, cage-type, dumbbell-shape, and ladder-structured ones [2-9]. Among them, cage-type POSSs have been investigated extensively, owing to their well-defined nano-sized structures. Polymers possessing POSS cages as structural subunits can have special properties, such as improved mechanical and thermal properties [10-13], and therefore the synthesis of such polymers has constantly been an attractive research direction.

POSS-cage-containing polymers can be designed and prepared in several different topologies depending on the synthesis methodologies, among which the controlled radical polymerization has received most attention. The alkene functional groups are first introduced into POSS cage molecules through organic synthesis, and then polymerization techniques such as RAFT [14-17], ATRP [18,19], ROMP [20-22] allow subsequent generation of POSS-cage-containing polymers either by themselves or with other alkene monomers. Other ways, such as coupling reactions, have also been utilized for a similar purpose. Depending on the number of functional groups within one POSS cage, the morphology of the resulting polymers can be radically different. The POSS cages with two connecting points can be incorporated into linear polymer backbones, and they can be attached sidelong of linear polymers if only one connecting point is present [23-28]. POSS cages with multiple connecting points usually lead to cross-linked polymers, and the special geometry of POSSs can make the polymers possess interesting permanent porosity [29-31]. 
However, for POSS cage monomers with multiple connecting points, it is also possible to generate soluble hyperbranched structures with POSS-cage incorporated either in the core or on the periphery with judicious design, and such polymers are of particular interest since they offer unique properties such as low viscosity and internal free space, not seen in other morphologies. Up to now, most hyperbranched POSS-cage-containing polymers have been prepared involving carbon-based chemistry in the backbone construction [32]. However, the presence of $\mathrm{C}-\mathrm{O}$ or $\mathrm{C}-\mathrm{N}$ bonds in the backbone can significantly deteriorate the thermal performance of such polymers even in the presence of POSS subunits. Polysiloxanes are special polymers which only involve strong $\mathrm{Si}-\mathrm{O}$ bonds in the backbone, and therefore can exhibit much improved thermal performance. In this regard, of particular interest to us is the hyperbranched POSS-cage-containing polysiloxanes (HPCCPs), since it offers all-siloxane type cage polymers, which can be possibly used in harsh conditions.

HPCCPs have been prepared from octakis(dimethylsiloxy) octasilsesquioxane $\left(\mathrm{Q}_{8}{ }^{\mathrm{DMS}}\right)$, which reacts with themselves or with phenylsiloxanediols through dehydrogenative polycondensation under certain catalysts [33-35]. However, the insufficient catalysis efficiency in these reactions failed to produce polymers with high molecular weight. As the reaction goes, the concentration of reactive groups gradually drops to a level when active ends of low molecular weight (MW) polymers stop to react further and the polymer stop to grow. The MW is usually between 2 to $50 \times 10^{3}$ dalton. In addition, the choice of starting materials is quite limited due to necessary use of unstable siloxanediols, resulting a poor structural variety in the final polymer. Therefore, there currently is a need to develop new methodologies to access high MW and rich structural tuneability in HPCCPs, which can endow more opportunities to such siloxane-based materials than what they have now.

We previously reported the synthesis of hyperbranched polysiloxanes through de-alkyl coupling reaction between hydrosilane and tetraethylorthosilicate catalyzed by $\left.\mathrm{B}_{(} \mathrm{C}_{6} \mathrm{~F}_{5}\right)_{3}$ [36]. The high efficiency obtained encouraged us to investigate the possibility of combining $\mathrm{Q}_{8}{ }^{\mathrm{DMS}}$ with different dialkoxysilanes to generate HPCCPs. The high catalysis efficiency can promise chances to achieve high MW polymers, while the redundant kinds of dialkoxysilanes available either commercially or through simple synthesis provide opportunities for structural variety.

Herein, we would like to report our work in this direction on the synthesis of HPCCP with ultra-high MW $\left(>1 \times 10^{6}\right)$ by use of simple starting materials. The great size and available functional groups enabled core environment modification and subsequent encapsulation of transition metal nanoparticles within the polymer.

\section{Experimental}

\subsection{Materials and Methods}

All reactions were carried out under an air atmosphere in oven-dried single-neck round bottom flasks with magnetic stirring bars. Tris(pentafluorophenyl)borane, $\mathrm{Q}_{8}{ }^{\mathrm{DMS}}$, cyclohexane, diphenyldimethoxysilane, 3-chloropropylmethyldiethoxysilane, triethyl phosphite, tetrahydrofuran, methanol, toluene, hexadecyltrimethylammonium chloride, sodium tetrahydroborate $\left(\mathrm{NaBH}_{4}\right)$, silver nitrate $\left(\mathrm{AgNO}_{3}\right)$, sodium chloropalladate $\left(\mathrm{Na}_{2} \mathrm{PdCl}_{4}\right)$, and sodium tetrachloroaurate(III) dehydrate $\left(\mathrm{NaAuCl}_{4} \cdot 2 \mathrm{H}_{2} \mathrm{O}\right)$ were purchased from InnoChem, Inc., Pudong, China, and then used without any further purification. Water was distilled before use. ${ }^{1} \mathrm{H}-\mathrm{NMR}(400 \mathrm{MHz})$ spectra were recorded in $\mathrm{CDCl}_{3}$ on a Bruker Avanced $400 \mathrm{NMR}$ spectrometer. The ${ }^{1} \mathrm{H}-\mathrm{NMR}$ spectra were referenced to residual solvent signals at $7.26 \mathrm{ppm}\left(\mathrm{CHCl}_{3}\right) .{ }^{29} \mathrm{Si}-\mathrm{NMR}$ spectra was recorded in $\mathrm{CDCl}_{3}$ with a $60 \mathrm{~s}$ delay at $120 \mathrm{MHz}$. Gel-permeation chromatography (GPC) analysis was carried out on a Viscotek GPC/SEC 270 max with RI/UV/viscosity/light scattering as the detectors, and toluene was used as the eluent. Dynamic light scattering (DLS) tests were carried out in THF with the concentration of $8 \mathrm{mg} / \mathrm{mL}$ on the Zetasizer Nano ZS90. For Transmission Electron Microscopy (TEM) imaging, the dichloromethane solution of relative samples (sodium chloropalladate/silver nitrate/sodium tetrachloroaurate(III) dehydrate) with the concentration of $0.001 \mathrm{mmoL} / \mathrm{mL}$ was drop-coated on the copper film, and were 
then imaged after drying in the air by HT7700 apparatus of HITACHI with the accelerating voltage of $200 \mathrm{kv}$. Thermogravimetric analysis (TGA) was conducted by TA SDT-Q600 either in the air or nitrogen at the heating speed of $10{ }^{\circ} \mathrm{C} / \mathrm{min}$ with around $5 \mathrm{mg}$ sample. Fourier Transform Infrared Spectroscopy (FTIR) was done by Nicolet 6700 FT-IR spectrometer on the KBr pallet with the transmission mode in the sample concentration range of $0.1-1.0 \mathrm{wt} \%$. Single-crystal X-ray diffraction was conducted on Bruker Apex II single-crystal X-ray diffraction with Mo source.

\subsection{Synthesis of Polymer 1}

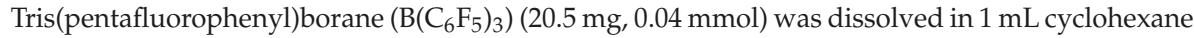
in a $10 \mathrm{~mL}$ two-neck flask equipped with a stirring bar. A mixture of $\mathrm{Q}_{8}{ }^{\mathrm{DMS}}(0.509 \mathrm{~g}, 0.5 \mathrm{mmol})$ and diphenyldimethoxysilane $(0.489 \mathrm{~g}, 2 \mathrm{mmol})$ in $6.25 \mathrm{~mL}$ cyclohexane was slowly added to the catalyst solution. After $1 \mathrm{~h}$, the solvent was removed using rotovap to obtain a white solid powder which was then dissolved in a small amount of tetrahydrofuran and precipitated out using methanol. The product was then washed repeatedly with methanol. After washing, the solid was dried under an oil pump to give white solid powder (polymer 1, $0.586 \mathrm{~g}$, yield $62 \%$ ).

\subsection{Synthesis of the Crystal $\mathbf{1}$}

$\mathrm{B}\left(\mathrm{C}_{6} \mathrm{~F}_{5}\right)_{3}(40 \mathrm{mg}, 0.0781 \mathrm{mmol})$ was dissolved in $50 \mathrm{~mL}$ cyclohexane at $50{ }^{\circ} \mathrm{C}$ in a $250 \mathrm{~mL}$ two-neck flask. A mixture of $\mathrm{Q}_{8}{ }^{\mathrm{DMS}}(6.3625 \mathrm{~g}, 6.25 \mathrm{mmol})$ and diphenyldimethoxysilane $(6.109 \mathrm{~g}, 25 \mathrm{mmol})$ in $40 \mathrm{~mL}$ cyclohexane was added to the catalyst solution. After addition, the reaction mixture was stirred for another $2 \mathrm{~h}$. The reaction mixture was then filtered, and the filtrate (about $100 \mathrm{~mL}$ ) was slowly added to $\mathrm{MeOH}(300 \mathrm{~mL})$. The solution was then transferred to a refrigerator, and kept overnight. The crystals were then collected through filtration (crystal 1, $0.44 \mathrm{~g}$, yield 3.5\%).

\subsection{Synthesis of Polymer 2}

$\mathrm{B}\left(\mathrm{C}_{6} \mathrm{~F}_{5}\right)_{3}(20 \mathrm{mg}, 0.04 \mathrm{mmol})$ was dissolved in $10 \mathrm{~mL}$ cyclohexane in a $50 \mathrm{~mL}$ two-neck flask equipped with a stirring bar. A mixture of $\mathrm{Q}_{8}{ }^{\mathrm{DMS}}(0.508 \mathrm{~g}, 0.5 \mathrm{mmol})$ and 3-chloropropylmethyldiethoxysilane $(0.42$ $\mathrm{g}, 2 \mathrm{mmol}$ ) in $2.5 \mathrm{~mL}$ cyclohexane was slowly added to the catalyst solution. After $1.5 \mathrm{~h}$, the solvent was removed using a rotovap to obtain a white solid powder which was then dissolved in a small amount of tetrahydrofuran and precipitated using methanol. The product was then washed repeatedly with methanol. After washing, the solid was dried under an oil pump as white powder (polymer 2, $0.13 \mathrm{~g}$, yield $16 \%$ ).

\subsection{Synthesis of Polymer 3}

Synthesis of polymer 3: polymer $2(100 \mathrm{mg})$ and triethyl phosphite $(0.16 \mathrm{~g}, 0.98 \mathrm{mmol})$ were mixed and heated at $180{ }^{\circ} \mathrm{C}$. After $48 \mathrm{~h}$, the reaction mixture was concentrated and dissolved in 1 $\mathrm{mL}$ tetrahydrofuran. Excessive $\mathrm{MeOH}$ was added to induce precipitation. After additional $\mathrm{MeOH}$ washing, the white solid was dried under an oil pump (polymer 3, $0.025 \mathrm{~g}$, yield 65\%).

\subsection{Transition Metal Nanoparticle Encapsulation by Polymer 3}

Gold nanoparticle encapsulation: a solution of $\mathrm{NaAuCl}_{4}(0.95 \mathrm{mg}, 0.0024 \mathrm{mmol})$ in deionized $\mathrm{H}_{2} \mathrm{O}$ $(1 \mathrm{~mL})$ was added to the solution of hexadecyltrimethylammonium chloride $(3.84 \mathrm{mg}, 0.012 \mathrm{mmol})$ in $\mathrm{CH}_{2} \mathrm{Cl}_{2}(1 \mathrm{~mL})$. The resulting mixture was stirred until $\mathrm{Au}$ (III) was transferred into the organic phase ( $\sim 60 \mathrm{~min})$. A solution of polymer $3(5 \mathrm{mg})$ in $\mathrm{CH}_{2} \mathrm{Cl}_{2}(1 \mathrm{~mL})$ was added. Subsequently, the gold salts in the solution was reduced by addition of an aqueous solution of $\mathrm{NaBH}_{4}(2.7 \mathrm{mg}, 0.072 \mathrm{mmol})$ in deionized $\mathrm{H}_{2} \mathrm{O}(1 \mathrm{~mL})$. The resulting organic layer was in mauve. The $\mathrm{CH}_{2} \mathrm{Cl}_{2}$ layer was separated and used for transmission electron microscopy (TEM) analysis.

Silver nanoparticle encapsulation: a solution of $\mathrm{AgNO}_{3}(1 \mathrm{mg}, 0.00388 \mathrm{mmol})$ in deionized $\mathrm{H}_{2} \mathrm{O}$ $(1 \mathrm{~mL})$ was added to the solution of hexadecyltrimethylammonium chloride $(6.21 \mathrm{mg}, 0.0194 \mathrm{mmol})$ 
in $\mathrm{CH}_{2} \mathrm{Cl}_{2}$ (1 mL). The resulting mixture was stirred until $\mathrm{Ag}$ (I) was transferred into organic phase ( $\sim 60 \mathrm{~min})$. A solution of polymer $3(1.97 \mathrm{mg})$ in $\mathrm{CH}_{2} \mathrm{Cl}_{2}(1 \mathrm{~mL})$ was added. Subsequently, the silver salts in the solution was reduced by addition of an aqueous solution of $\mathrm{NaBH}_{4}(4.4 \mathrm{mg}, 0.1164 \mathrm{mmol})$ in deionized $\mathrm{H}_{2} \mathrm{O}(1 \mathrm{~mL})$. The resulting organic layer was in earthy yellow. The $\mathrm{CH}_{2} \mathrm{Cl}_{2}$ layer was separated and used for TEM analysis.

Palladium nanoparticle encapsulation: a solution of $\mathrm{Na}_{2} \mathrm{PdCl}_{4}(0.59 \mathrm{mg}, 0.002 \mathrm{mmol})$ in deionized $\mathrm{H}_{2} \mathrm{O}(1 \mathrm{~mL})$ was added to the solution of hexadecyltrimethylammonium chloride $(3.2 \mathrm{mg}, 0.01 \mathrm{mmol})$ in $\mathrm{CH}_{2} \mathrm{Cl}_{2}(1 \mathrm{~mL})$. The resulting mixture was stirred until $\mathrm{Pd}$ (II) was transferred into organic phase ( $\sim 60 \mathrm{~min})$. A solution of polymer $3(1.01 \mathrm{mg})$ in $\mathrm{CH}_{2} \mathrm{Cl}_{2}(1 \mathrm{~mL})$ was added. Subsequently the palladium salts in the solution was reduced by addition of an aqueous solution of $\mathrm{NaBH}_{4}(2.3 \mathrm{mg}, 0.06 \mathrm{mmol})$ in deionized $\mathrm{H}_{2} \mathrm{O}(1 \mathrm{~mL})$. The resulting organic layer was in black-brown. The $\mathrm{CH}_{2} \mathrm{Cl}_{2}$ layer was separated and used for TEM analysis.

\section{Results and Discussion}

\subsection{Synthesis of Polymer 1}

As shown in Scheme 1, polymer 1 was synthesized by the reaction of $\mathrm{Q}_{8}{ }^{\mathrm{DMS}}$ with diphenyldimethoxysilane in cyclohexane, using tris(pentafluorophenyl) borane as the catalyst. The reaction proceeded very fast, and upon addition of reactants into the catalyst solution, gas bubbles formed immediately, indicating the releasing of methane gas and formation of $\mathrm{Si}-\mathrm{O}-\mathrm{Si}$ bond. The observed high efficiency of so-called Piers-Rubinsztajn reaction is consistent with previous reports [37,38]. The obtained product after precipitation purification is a white solid and dissolves in common solvents such as THF, dichloromethane, and cyclohexane very well, but not in methanol.
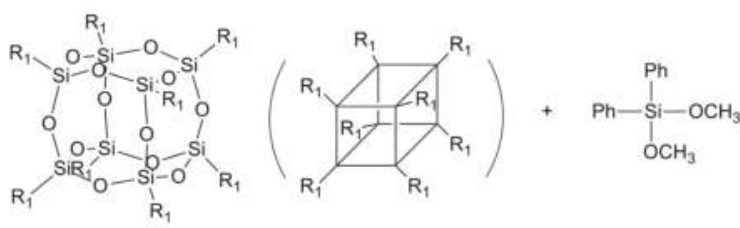

$R_{1}=-O-S i\left(M e_{2}\right)-H$

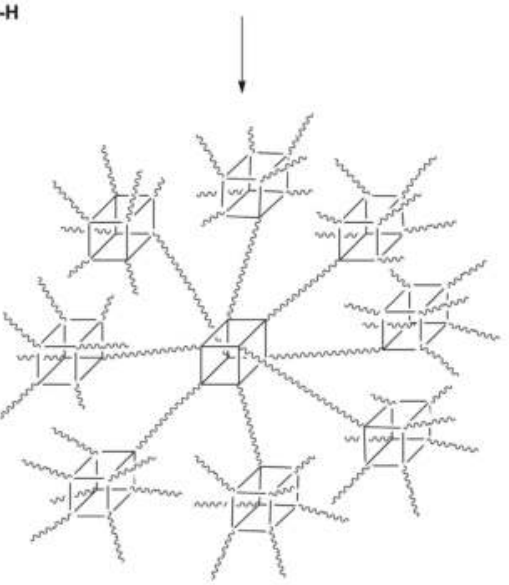

Polymer 1

Scheme 1. Reaction scheme to prepare polymer 1. 
The absolute molecular weight of polymer 1 was measured with GPC equipped with light scattering and viscosity detector. Surprisingly the MW was found to be as large as around 4.1 million (weight-averaged), corresponding to around 2200 POSS subunits. The polydispersity index (PDI) was around 1.2 (Figure S9 and Table S1). The giant size of the polymer is also confirmed by dynamic light scattering experiments, which indicates the averaged diameter of around $8 \mathrm{~nm}$ for a single molecule of polymer 1 (Figure 1D). The huge molecular weight is unprecedented for any POSS-cage containing polysiloxanes. Polymer $\mathbf{1}$ can be possibly regarded as one example of single molecular particles given its hyperbranched structures, and their exact solution behavior, whether adopting a fixed geometry and shape or randomly folded structures, remains as a curiosity which probably needs further investigation with respect to effect of incorporated rigid POSS cages.

A

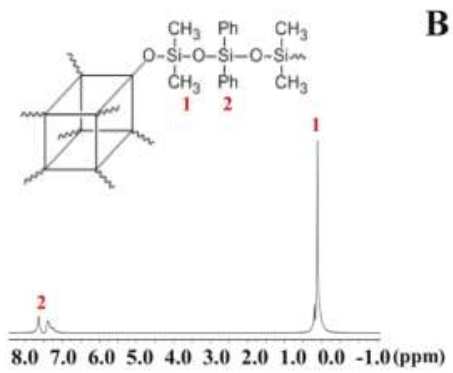

C

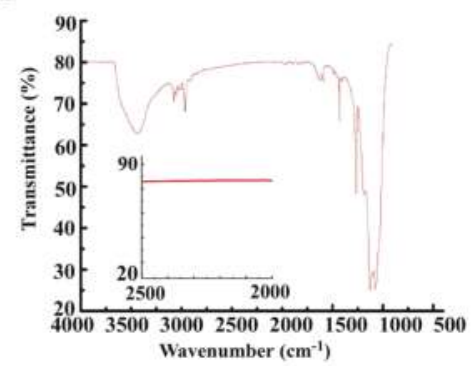

B
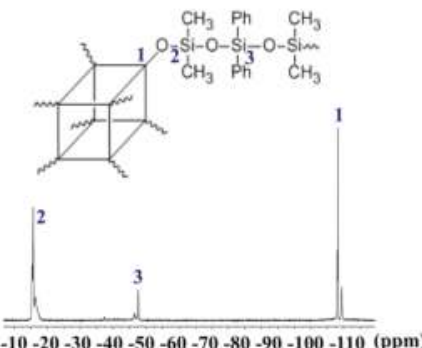

D

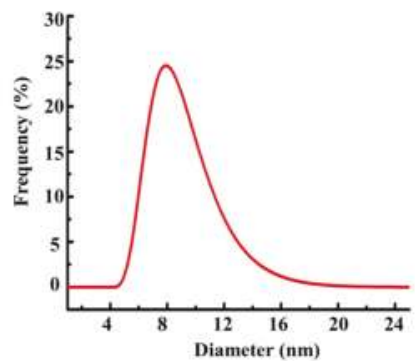

Figure 1. (A) the ${ }^{1} \mathrm{H}-\mathrm{NMR}$ of polymer 1 in $\mathrm{CDCl}_{3}$; (B) the ${ }^{29} \mathrm{Si}-\mathrm{NMR}$ of polymer $\mathbf{1}$ in $\mathrm{CDCl}_{3}$; The $\mathrm{Q}$ region was contaminated by the $\mathrm{Q}$ Si atoms in the NMR tube, and the spectrum was then flatten particularly in that region for the sake of clarity. (C) the IR of polymer 1 between 2000 and $2500 \mathrm{~cm}^{-1}$; (D) the dynamic light scattering (DLS) of polymer 1, showing the diameter of around $8 \mathrm{~nm}$.

We attributed the formation of high molecular weight polymer $\mathbf{1}$ to the high efficiency of the Piers-Rubinsztajn reaction, which forces the full consumption of the hydride and methoxy groups, and therefore the continuing growing of the polymer into a giant size. The IR spectrum of polymer 1 (Figure 1C) shows the absence of any peak between 2250 and $2100 \mathrm{~cm}^{-1}$, indicating the full consumption of $\mathrm{Si}-\mathrm{H}$ groups in polymer $\mathbf{1}$. The complete absence of terminating hydride is also confirmed by ${ }^{1} \mathrm{H} /{ }^{29} \mathrm{Si}-\mathrm{NMR}$ in Figure $\left.1 \mathrm{~A}, \mathrm{~B}\right)$, which also indicates the absence of any methoxy leftover within polymer 1, detailed analysis in Figures S3 and S4. These results are highly similar to our previous report about the reaction between tetramethyldisiloxane and tetraethylorthosilicate, in which all hydride and methoxy groups fully react to form a hyperbranched polymer [36].

In addition to the proposed hyperbranched structure as shown in Scheme 1, it is also reasonable to expect cross-linked particles to form during the reaction. However, the absence of precipitation after the reaction indicated high proclivity of the reaction to formation of the hyperbranched version instead of the cross-linked one. The full consumption of all reactive groups, as discussed above, combined 
with the good solubility of polymer $\mathbf{1}$, strongly suggests the assumption that significant intramolecular cyclization reaction must happened at the later stage of reaction. As the reaction goes, the concentration of small molecular reactants will drop to a level where the intramolecular reaction overwhelms the intermolecular reaction, and the intramolecular cyclization will happen predominantly to close up the polymer, which is then inert for further growth.

In order to prove this assumption, we carried out the reaction in a dilute concentration in order to induce the intramolecular cyclization reaction to happen at a much earlier stage in the hope of obtaining well-characterized small molecule. Luckily, we successfully obtained the single crystal structure of smallest version of polymer $\mathbf{1}$ (crystal 1, around 3.5\%), in which all adjacent hydride groups react intramolecularly with diphenyldimethoxysilane to close into a loop on the side of the POSS cage, as shown in Scheme 2. This small molecule forms nice block-like crystal to permit us conduct single-crystal X-ray diffraction on it. The unit cell of crystal 1 belongs to the triclinic group, and there is one POSS molecule for each unit cell (See Table S2 for unit cell parameters). The relative ${ }^{1} \mathrm{H}$ - and ${ }^{29} \mathrm{Si}-\mathrm{NMR}$ spectra (Figures S1 and S2) confirmed the structure. As shown in Figure 2, the full preservation of the POSS cage is consistent with the benign nature of the $\left.\mathrm{B}_{(} \mathrm{C}_{6} \mathrm{~F}_{5}\right)_{3}$ catalyst towards the $\mathrm{Si}-\mathrm{O}$ bonds, and the $\mathrm{Si}-\mathrm{O}$ bonds are usually uninterrupted during the Pier-Rubinsztajn reaction as shown by Brook et al. [39]. The structural rearrangement through the cleavage and reformation of $\mathrm{Si}-\mathrm{O}$ bonds by a lot of catalysts has been troublesome [29] and this problem seems to absent in our approach, therefore maximizing the structural fidelity of generated polymers according to the design rules.

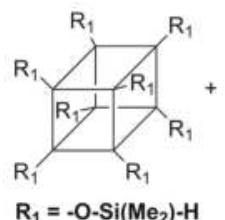<smiles>CO[Si](OC)(c1ccccc1)c1ccccc1</smiles>

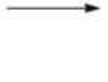

\section{crystal 1}

)$=-0-\mathrm{Si}\left(\mathrm{Me}_{2}\right)-\mathrm{O}-\mathrm{Si}\left(\mathrm{Ph}_{2}\right)-\mathrm{O}-\mathrm{Si}\left(\mathrm{Me}_{2}\right)-\mathrm{O}$ -

Scheme 2. Reaction scheme of crystal 1.

A

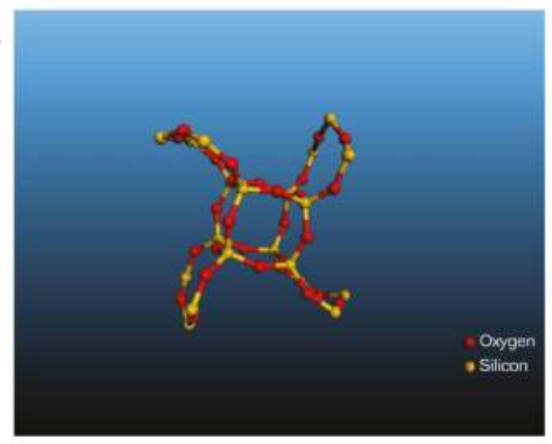

B

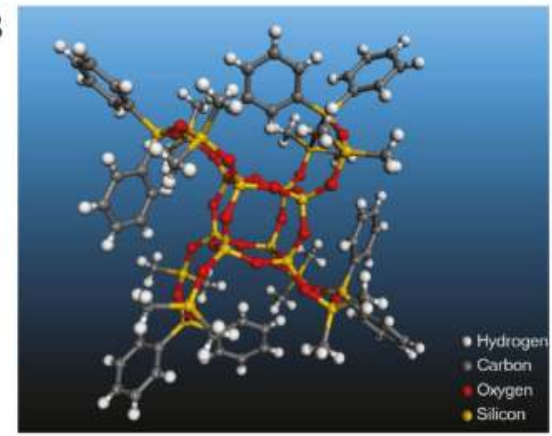

Figure 2. Images of the single crystal structure of crystal $\mathbf{1}$ without (A) and with (B) carbon and hydrogen elements.

The structural feature of crystal 1 sheds light into the structure of polymer 1 . As shown in Figure 2, the cyclopentasiloxane rings on the side of POSS cage in crystal $\mathbf{1}$ are all significantly distorted to one direction presumably due to the crowded surface of the POSS cage, and therefore the cyclopentasiloxane ring in crystal 1 should be more constrained than regular ones [40-43]. In other 
words, there will be lesser chance for the polymer to close up and stop growing due to the crowded space, and this facilitates the formation of high MW polymers. Only when polymer grows to a giant size, the intramolecular cyclization may happen due to the significantly reduced chance for intermolecular reaction with other reactive small molecules, and then the polymers stop growing. Combined with the absence of $\mathrm{SiH}$ or SiOMe groups, as supported by ${ }^{29} \mathrm{Si}-$ and ${ }^{1} \mathrm{H}-\mathrm{NMR}$, the end groups of polymer 1 are estimated to be mostly intramolecular loops. However it has to be pointed out that the possibility of other kind of intramolecular cyclization with polymer $\mathbf{1}$, other than the one shown in Figure 2 cannot be completely ruled out.

\subsection{TGA of Polymer 1}

As expected, TGA indicated a very good thermal performance with $5 \%$ weight loss temperature $\left(\mathrm{TD}_{5}\right)$ at $473{ }^{\circ} \mathrm{C}$ in nitrogen. The char value is around $80 \%$ even up to $900{ }^{\circ} \mathrm{C}$ (Figure $3 \mathrm{~A}$ ). In comparison, the $\mathrm{TD}_{5}$ for PDMS is only around $370{ }^{\circ} \mathrm{C}$ (in nitrogen) with a char value of below $40 \%$ at around $550{ }^{\circ} \mathrm{C}$ [44]. Apparently, polymer 1, with an all-Si-O backbone, performs much better than regular PDMS elastomers during aging. The role of POSSs may be explained by the facilitated formation of protective silicon oxide layer on the surface during heating, and therefore reduce the weight loss during aging. The presence of phenyl groups in polymer $\mathbf{1}$ is also beneficial, since they may help to form dense carbon layer upon heat, and this then adds up another mechanism of protection $[45,46]$.
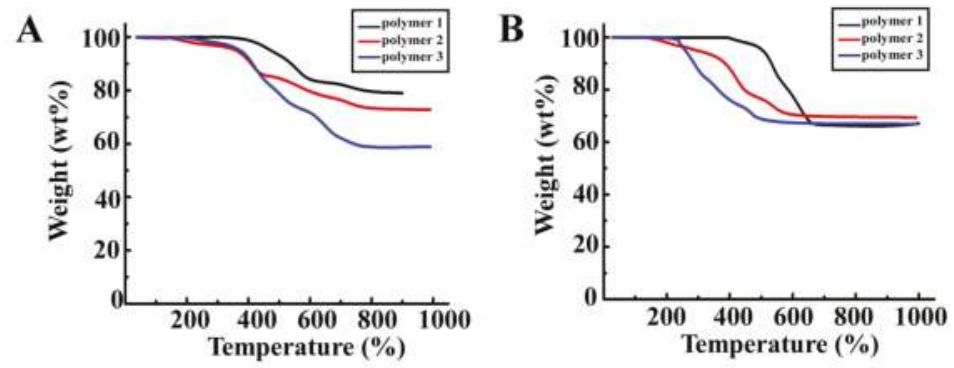

Figure 3. TGA plots of the polymer 1, polymer 2 and polymer 3 under the $\mathrm{N}_{2}$ (A) and air (B) atmosphere respectively.

Interestingly, the $\mathrm{TD}_{5}$ value is $503{ }^{\circ} \mathrm{C}$ in air (Figure 3B), presumably due to the accelerated formation of silicon oxide layer in the air atmosphere at an earlier stage than in nitrogen environment. However, the char value drops significantly down to around $65 \%$ at $900{ }^{\circ} \mathrm{C}$ and this may be explained by the reduced efficiency in the formation of carbon char by the phenyl groups in air at such high temperature, and they may instead burn up with oxygen in the air to generate volatile compounds. The performance of polymer 1 during heating in the air in terms of $\mathrm{TD}_{5}$ and char value is consistent with the above assumption of the roles of POSSs and phenyl groups. Polymer $\mathbf{1}$ is by far one of the most thermally stable polysiloxanes ever reported [47-49].

The presence of phenyl groups is crucial for high thermal performance in both nitrogen and air atmosphere. As shown in Figure 3A,B, neither polymer 2 or 3 failed to maintain as good performance as polymer 1 . In nitrogen atmosphere, the $\mathrm{TD}_{5}$ values for polymers 2 and 3 are $360^{\circ} \mathrm{C}$ and $365^{\circ} \mathrm{C}$, respectively, and they are around $100^{\circ} \mathrm{C}$ lower than that of polymer $1\left(473^{\circ} \mathrm{C}\right)$. This significant difference reflects the pivot role of phenyl by forming dense carbon layers to prevent degradation. Char values up to $900{ }^{\circ} \mathrm{C}$ are then $73 \%$ and 59\% respectively for polymers 2 and 3, also smaller than that of polymer $\mathbf{1}(80 \%)$.

The performance of polymers 2 and 3 in air during heating are also inferior to that of polymer 1, but in a slightly different way. In air, the $\mathrm{TD}_{5}$ values for polymers 2 and 3 are 294 and $259{ }^{\circ} \mathrm{C}$, respectively, more than $200{ }^{\circ} \mathrm{C}$ lower than that of polymer $1\left(503{ }^{\circ} \mathrm{C}\right)$. The exaggeratedly weak performance in 
terms of $\mathrm{TD}_{5}$ values are due to the high reactivity of oxygen molecules in the air towards the organic components other than phenyl groups in the polymers. The importance of the dense carbon layer formed by phenyl groups (below $500{ }^{\circ} \mathrm{C}$ ) are more obvious in air than in nitrogen, since oxygen molecules react much more readily with organic groups than nitrogen molecules, and the loss of the barrier of dense carbon layer in polymers 2 and $\mathbf{3}$ lead to more weight loss in air. In fact, the performance of polymers in air is actually more related to the realistic use of fire-retardant materials than in nitrogen. The almost unchanged char values up to $900{ }^{\circ} \mathrm{C}$, even slighter higher in polymer $2(69 \%)$ and polymer $3(67 \%)$, again confirms with above conclusion that all organic groups, either phenyl or 3-chloropropyl or phosphonate groups, burn up in the air at such a high temperature, and the remaining weight are mostly ascribed to the $\mathrm{Si} / \mathrm{O}$ backbone, which are almost the same for polymers $\mathbf{1}, \mathbf{2}$, and 3 , with the latter two actually slightly larger.

\subsection{Functional Group Variety and Application in Encapsulation of Inorganic Nanoparticles}

The use of diphenyldimethoxysilane instead of diphenylsilanediol in the synthesis of polymer 1 represents the significant advantage of our approach since there are broad choice of dialkoxysilanes available either commercially or through easy synthesis. In sharp contrast, the relative diol compounds used in previous approaches are mostly limited to diphenylsilanediol and silanol-terminated polydimethylsiloxane. For the demonstration of opportunities for our polymers to advance into other areas, we then changed the starting alkoxysilane to 3-chloropropylmethyldiethoxysilane, with the hope that chloropropyl group can be chemically modified subsequently for specific purposes.

The reaction between 3-chloropropylmethyldiethoxysilane and $\mathrm{Q}_{8}{ }^{\mathrm{DMS}}$ was conducted similarly as the procedure for polymer $\mathbf{1}$, and the resulting polymer $\mathbf{2}$ has a MW of 1.1 million (weight-averaged), confirming generality of this reaction (Figure S10 and Table S1). ${ }^{1} \mathrm{H}$ and ${ }^{29} \mathrm{Si}$ spectra (Figures S5 and $\mathrm{S6}$ ) indicated full consumption of all $\mathrm{Si}-\mathrm{H}$ and $\mathrm{Si}-\mathrm{OEt}$ groups, also supported by IR (Figure S8). The size of polymer 2 (Figure 4) was close to that of polymer $\mathbf{1}$ (Figure 1D), and both were around $9 \mathrm{~nm}$. The PDI of polymer 2, however, is around 2.3, much bigger than that of polymer $\mathbf{1}$, which is only 1.2. In fact, close inspection of the GPC curve of polymer 2 (Figure S10) revealed the bimodal nature of it while polymer 1 only exhibited a single peak (Figure S9), and this is consistent with the PDI difference. While exact cause for the broad dispersity of polymer 2 probably needs future analysis of its microstructure to distinguish different reaction pathways, the size and electronic structure of 3-chloropropyl groups is highly possible to play a role. In addition, the effect of substitution groups on the PDI was also observed in our previous report [38].

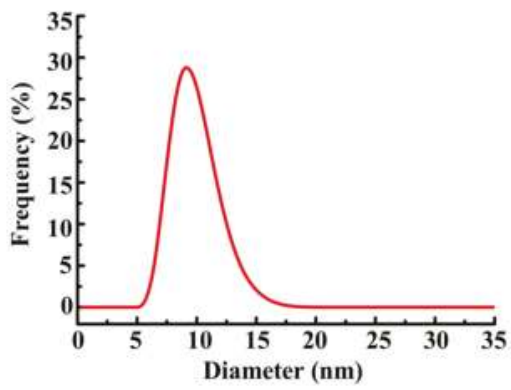

Figure 4. DLS of the polymer 2, showing the diameter of around $9 \mathrm{~nm}$.

Polymer 2 was then modified further through Michaelis-Arbuzov reaction to introduce phosphonate groups (as shown in Scheme 3), since the phoshonate groups have strong affinity to a wide range of transition metals and will be helpful in preparing hybrid materials. Refluxing polymer 2 with triethylphosphite resulted in around 59\% incorporation of phosphonate groups (as shown in 
Figure 5, detailed analysis in Figure S7), and the obtained polymer 3 dissolves in various solvents very well, but still not in methanol, probably because the polar phosphonate groups are buried within the polymer, preventing effective interaction with the methanol solvent molecules.

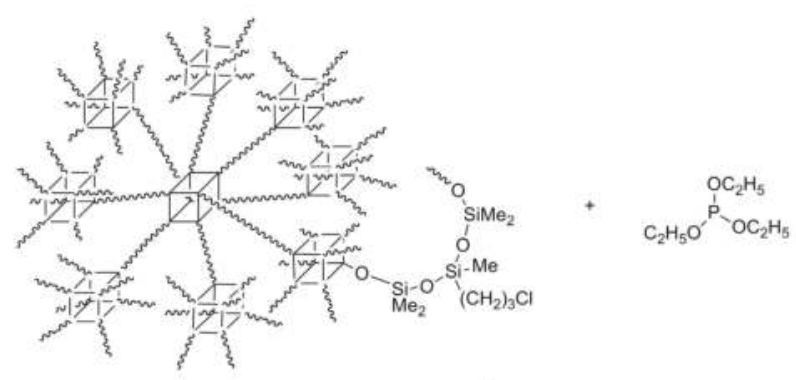

Polymer 2

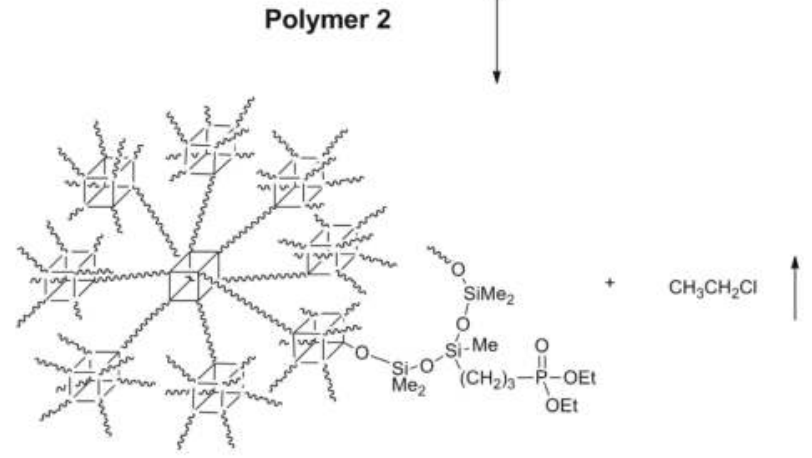

Polymer 3

Scheme 3. Reaction scheme to prepare polymer 3.
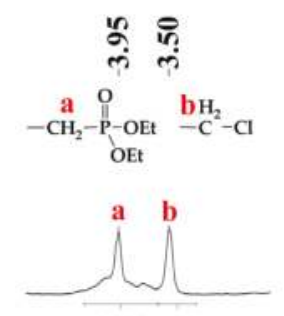

3.641 .91

\section{$\begin{array}{llll}4.5 & 4.0 & 3.5 & 3.0\end{array}(\mathrm{ppm})$}

Figure 5. ${ }^{1} \mathrm{H}-\mathrm{NMR}$ of polymer 3 and relative assignment of the peaks. The conversion of the chloropropyl groups into phosphonate-propyl groups was determined according to the integral ratio (Figure S7).

Dendritic polymers, either hyperbranched ones or dendrimers, have been proved useful in stabilizing transition nanoparticles [50-53], which are of high importance in organic catalysis. The presence of rigid POSS subunits are helpful for forming internal porosity to host such nanoparticle, especially with the assistance of polar phosphonate groups introduced in polymer 3 . In fact, polymer 3 turned to be a welcome host for various transition metal nanoparticles (NPs) even without the need 
of hydrolysis of the phosphonate groups into relative acid. For example, reduction of $\mathrm{NaAuCl}_{4}$ by the $\mathrm{NaBH}_{4}$ in the presence of polymer 3 (Scheme 4) resulted in a clear and mauve solution without any precipitation formed. TEM analysis clearly revealed the gold NPs around $4.4 \mathrm{~nm}$ (Figure 6A), consistent with the size of a single polymer and therefore supporting the encapsulation of the NPs within the core of hyperbranched polymer 3 . Simply replacing the gold salts with silver or palladium ones resulted in similar encapsulated NPs by polymer 3, as evidenced by TEM. The average sizes for $\mathrm{Ag}$ and Pd NPs are $5 \mathrm{~nm}$ and $4.3 \mathrm{~nm}$, respectively (Figure 6B,C). Interestingly, for Ag and Pd NPs, in addition to the random distribution (Figure S11), there exist unexpected circular assembly of these encapsulated particles, towards which we have no clear explanation yet. We suspect that the interaction between the exterior surface of the polymer may play a role in the formation of these novel shapes. The encapsulation of inorganic nanoparticles by polymer $\mathbf{1}$ adds up the library of polysiloxanes-stabilized nanoparticles [54-56]. Overall, the accommodation of different transition metal NPs is very attracting since they may find application in new catalysis reactions.
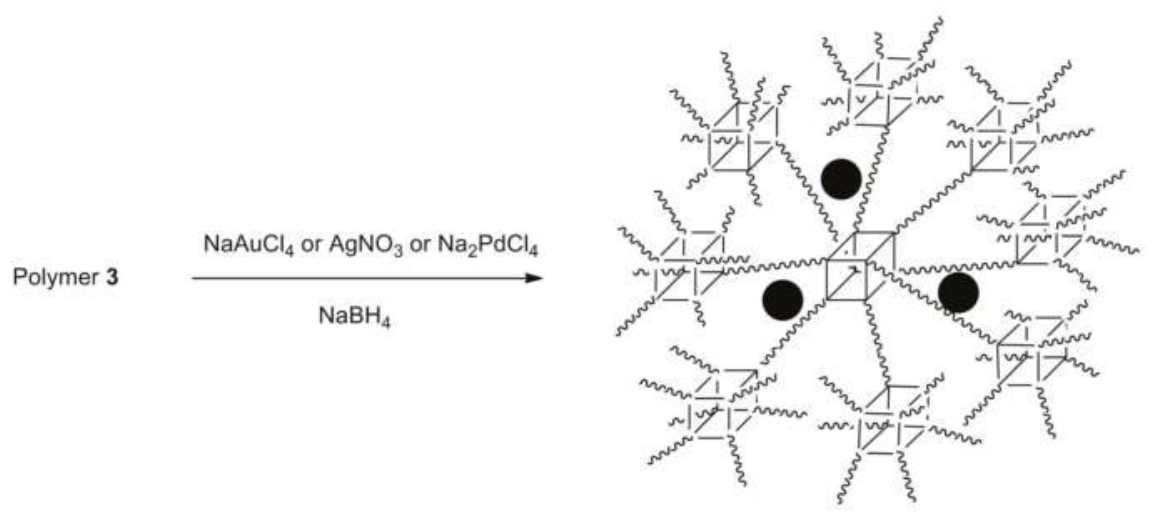

Scheme 4. Nanoparticle (NP) encapsulation by polymer 3 .
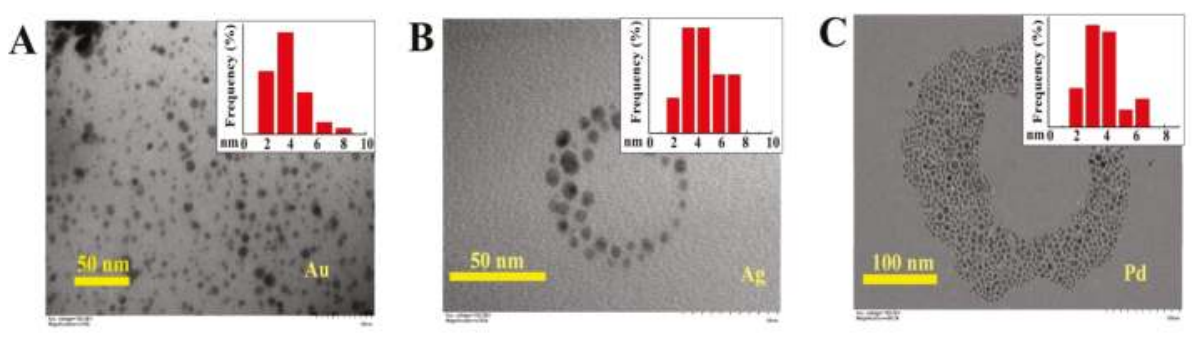

Figure 6. TEM images of nanoparticles of $\mathrm{Au}(\mathrm{A}), \mathrm{Ag}(\mathbf{B})$ and $\mathrm{Pd}(\mathrm{C})$ encapsulated within polymer 3. The distribution of particle sizes is shown in the insert of each image.

Compared to traditional polysiloxanes, which are mostly made from condensation reaction from dichlorosilane or ring opening reaction from cyclosiloxanes, our polymers present unique characters in terms of both high molecular weight and exchangeable functional groups. While traditional polysiloxanes are mostly limited to methyl version due to the fact that other functional groups bigger than methyl groups on the siloxane precursors tend to induce the formation of oligomers or short polymers, the polymers presented here are obviously free from these limitation, and will find more opportunities for utilization in a wide range of areas. 


\section{Conclusions}

We report in this manuscript for the first time the synthesis of POSS-cage-containing polysiloxanes with ultra-high molecular weight and structural tuneability. The great size of a single polymer around $10 \mathrm{~nm}$ in the hyperbranched morphology is rare. Given the possibility of introduction of various functional groups on the backbone while still maintaining high molecular weight, this series of polymers is expected to find usefulness in other areas. The introduction of phosphonate groups serves as an introductory example for possible application, and we are currently exploring further in this direction.

Supplementary Materials: The following are available online at http:/ /www.mdpi.com/2073-4360/10/5/496/s1, Figure S1: ${ }^{1} \mathrm{H}-\mathrm{NMR}$ of the crystal 1, Figure S2: ${ }^{29} \mathrm{Si}-\mathrm{NMR}$ of crystal 1, Figure S3: ${ }^{1} \mathrm{H}-\mathrm{NMR}$ of polymer 1, Figure S4: ${ }^{29} \mathrm{Si}-\mathrm{NMR}$ of polymer 1, Figure S5: ${ }^{1} \mathrm{H}-\mathrm{NMR}$ of polymer 2, Figure S6: $29 \mathrm{Si}-\mathrm{NMR}$ of polymer 2, Figure S7: ${ }^{1} \mathrm{H}-\mathrm{NMR}$ of polymer 3, Figure S8: IR of polymer 2, Figure S9: GPC of polymer 1, Figure S10: GPC of polymer 2, Figure S11: TEM images of nanoparticle coated polymers and corresponding particle size distribution, Table S1: GPC result summary of polymer 1 and polymer 2, Table S2: Unit cell parameters of crystal $\mathbf{1}$. The cif file of crystal $\mathbf{1}$ was deposited in CCDC database with the code of 1835605, which can be obtained free of charge from the Cambridge Crystallographic Data Center. The cif file was also provided in the supplementary materials.

Acknowledgments: This work was supported by Beihang University Research Fund 74004601 and the 111 Project (B14009). We thank the staff from BL17B at the Shanghai Synchrotron Radiation Facility for their assistance during data collection of the diffraction data of crystal 1.

Author Contributions: Y.L. conceived and designed the experiments; N.L. performed the experiments; N.L., Y.M. and J.Y. analyzed the data; Y.L., N.L. and Y.M. wrote the paper.

Conflicts of Interest: The authors declare no conflict of interest.

\section{References}

1. Scott, D.W. Thermal rearrangement of branched-chain methylpolysiloxanes. J. Am. Chem. Soc. 1946, 68, 356-358. [CrossRef]

2. Zheng, L.; Kasi, R.M.; Farris, R.J.; Coughlin, E.B. Synthesis and thermal properties of hybrid copolymers of syndiotactic polystyrene and polyhedral oligomeric silsesquioxane. J. Polym. Sci. Part A Polym. Chem. 2002, 40, 885-891. [CrossRef]

3. Baney, R.H.; Itoh, M.; Sakakibara, A.; Suzuki, T. Silsesquioxanes. Chem. Rev. 1995, 95, 1409-1430. [CrossRef]

4. Marcolli, C.; Calzaferri, G. Monosubstituted octasilasesquioxanes. Appl. Organomet. Chem. 1999, 13, $213-226$. [CrossRef]

5. Li, G.; Wang, L.; Ni, H.; Pittman, C.U. Polyhedral oligomeric silsesquioxane (POSS) polymers and copolymers: A review. J. Inorg. Organomet. Polym. 2001, 11, 123-154. [CrossRef]

6. Tereshchenko, T.A. Synthesis and application of polyhedral oligosilsesquioxanes and spherosilicates. Polym. Sci. 2008, 50, 249-262. [CrossRef]

7. Pielichowski, K.; Njuguna, J.; Janowski, B.; Pielichowski, J. Polyhedral oligomeric silsesquioxanes (POSS)-containing nanohybrid polymers. Adv. Polym. Sci. 2006, 201, 225-296. [CrossRef]

8. Abe, Y.; Gunji, T. Oligo- and polysiloxanes. Prog. Polym. Chem. 2004, 29, 149-182. [CrossRef]

9. Blanco, I.; Abate, L.; Bottino, F.A.; Bottino, P. Synthesis, characterization and thermal stability of new dumbbell-shaped isobutyl-substituted POSSs linked by aromatic bridges. J. Therm. Anal. Calorim. 2014, 117, 243-250. [CrossRef]

10. Fu, B.X.; Hsiao, B.S.; Pagola, S.; Stephens, P.; White, H.; Rafailovich, M.; Sokolov, J.; Mather, P.T.; Jeon, H.G.; Phillips, S. Structural development during deformation of polyurethane containing polyhedral oligomeric silsesquioxanes (POSS) molecules. Polymer 2001, 42, 599-611. [CrossRef]

11. Lee, A.; Lichtenhan, J.D. Thermal and viscoelastic property of epoxy-clay and hybrid inorganic-organic epoxy nanocomposites. J. Appl. Polym. Sci. 2015, 73, 1993-2001. [CrossRef]

12. Haddad, T.S.; Lichtenhan, J.D. Hybrid organic-inorganic thermoplastics: Styryl-based polyhedral oligomeric silsesquioxane polymers. Macromolecules 1996, 29, 7302-7304. [CrossRef]

13. Blanco, I.; Bottino, F.A. Effect of the substituents on the thermal stability of hepta cyclopentyl, phenyl substituted-polyhedral oligomeric silsesquioxane (hcp-POSS)/polystyrene (PS) nanocomposites. AIP Conf. Proc. 2012, 1459, 247-249. [CrossRef] 
14. Ramirez, S.M.; Diaz, Y.J.; Sahagun, C.M.; Duff, M.W.; Lawal, O.B.; Iacono, S.T.; Mabry, J.M. Reversible addition-fragmentation chain transfer (RAFT) copolymerization of fluoroalkyl polyhedral oligomeric silsesquioxane (F-POSS) macromers. Polym. Chem. 2013, 4, 2230-2234. [CrossRef]

15. Ye, Y.S.; Shen, W.C.; Tseng, C.Y.; Rick, J.; Huang, Y.J.; Chang, F.C.; Hwang, B.J. Versatile grafting approaches to star-shaped POSS-containing hybrid polymers using RAFT polymerization and click chemistry. Chem. Commun. 2011, 47, 10656-10658. [CrossRef] [PubMed]

16. Deng, Y.; Bernard, J.; Alcouffe, P.; Galy, J.; Dai, L. Nanostructured hybrid polymer networks from in situ self-assembly of RAFT-synthesized POSS-based block copolymers. J. Polym. Sci. Part A Polym. Chem. 2011, 49, 4343-4352. [CrossRef]

17. Zhang, Z.; Hong, L.; Gao, Y.; Zhang, W. One-pot synthesis of POSS-containing alternating copolymers by RAFT polymerization and their microphase-separated nanostructures. Polym. Chem. 2014, 5, 4534-4541. [CrossRef]

18. Wang, W.; Ding, W.L.; Yu, J.; Fei, M.; Tang, J.Y. Synthesis and characterization of a novel POSS/PS composite via ATRP of branched functionalized POSS. J. Polym. Res. 2012, 19, 9948-9953. [CrossRef]

19. Raus, V.; Čadová, E.; Starovoytova, L.; Janata, M. ATRP of POSS monomers revisited: Toward high-molecular weight methacrylate-POSS (co)polymers. Macromolecules 2014, 47, 7311-7320. [CrossRef]

20. Xu, W.; Kwon, Y.; Chung, C. Synthesis of novel block copolymers containing polyhedral oligomeric silsesquioxane (POSS) pendent groups via ring-opening metathesis polymerization (ROMP). Polymer 2007, 48, 6286-6293. [CrossRef]

21. Li, L.; Zhang, C.; Zheng, S. Synthesis of POSS-terminated polycyclooctadiene telechelics via ring-opening metathesis polymerization. J. Polym. Sci. Part A Polym. Chem. 2017, 55, 223-233. [CrossRef]

22. Gnanasekaran, D.; Reddy, B.S.R. Synthesis and characterization of nanocomposites based on copolymers of POSS-ONDI macromonomer and TFONDI: Effect of POSS on thermal, microstructure and morphological properties. Adv. Mater. Res. 2010, 123-125, 775-778. [CrossRef]

23. Wang, J.; Sun, J.; Zhou, J.; Jin, K.; Fang, Q. Fluorinated and thermo-crosslinked polyhedral oligomeric silsesquioxanes: New organic-inorganic hybrid materials for high performance dielectric application. ACS Appl. Mater. Interfaces 2017, 9, 12782-12790. [CrossRef] [PubMed]

24. Naka, K.; Masuoka, S.; Shinke, R.; Yamada, M. Synthesis of first- and second-generation imidazole-terminated POSS-core dendrimers and their $\mathrm{pH}$ responsive and coordination properties. Polym. J. 2012, 44, 353-359. [CrossRef]

25. Lichtenhan, J.D.; Otonari, Y.A.; Carr, M.J. Linear hybrid polymer building blocks: Methacrylate-functionalized polyhedral oligomeric silsesquioxane monomers and polymers. Macromolecules 1995, 28, 8435-8437. [CrossRef]

26. Hirai, T.; Leolukman, M.; Hayakawa, T.; Kakimoto, M.; Gopalan, P. Hierarchical nanostructures of organosilicate nanosheets within self-organized block copolymer films. Macromolecules 2008, 41, 4558-4560. [CrossRef]

27. Yu, C.B.; Ren, L.J.; Wang, W. Synthesis and self-assembly of a series of $n$ POSS- $b$-PEO block copolymers with varying shape anisotropy. Macromolecules 2017, 50, 3273-3284. [CrossRef]

28. Pramudya, I.; Rico, C.G.; Lee, C.; Chung, H. POSS-containing bioinspired adhesives with enhanced mechanical and optical properties for biomedical applications. Biomacromolecules 2016, 17, 3853-3861. [CrossRef] [PubMed]

29. Chaikittisilp, W.; Kubo, M.; Moteki, T.; Sugawaranarutaki, A.; Shimojima, A.; Okubo, T. Porous siloxaneorganic hybrid with ultrahigh surface area through simultaneous polymerizationdestruction of functionalized cubic siloxane cages. J. Am. Chem. Soc. 2011, 133, 13832-13835. [CrossRef] [PubMed]

30. Nischang, I.; Brüggemann, O.; Teasdale, I. Facile, Single-step preparation of versatile, high-surface-area, hierarchically structured hybrid materials. Angew. Chem. Int. Ed. 2011, 50, 4592-4596. [CrossRef] [PubMed]

31. Wang, D.; Xue, L.; Li, L.; Deng, B.; Feng, S.; Liu, H.; Zhao, X. Rational design and synthesis of hybrid porous polymers derived from polyhedral oligomeric silsesquioxanes via heck coupling reactions. Macromol. Rapid Commun. 2013, 34, 861-866. [CrossRef] [PubMed]

32. Naka, K.; Irie, Y. Synthesis of single component element-block materials based on siloxane-based cage frameworks. Polym. Int. 2017, 66, 187-194. [CrossRef]

33. Shioda, T.; Gunji, T.; Abe, N.; Abe, Y. Preparation and properties of polyhedral oligomeric silsesquioxane polymers. Appl. Organomet. Chem. 2011, 25, 661-664. [CrossRef] 
34. Gunji, T.; Igarashi, T.; Tsukada, S.; Abe, Y. Syntheses of cage octasilicate polymers. J. Sol-Gel Sci. Technol. 2017, 81, 21-26. [CrossRef]

35. Lichtenhan, J.D.; Vu, N.Q.; Carter, J.A.; Gilman, J.W.; Feher, F.J. Silsesquioxane-siloxane copolymers from polyhedral silsesquioxane. Macromolecules 1993, 26, 2141-2142. [CrossRef]

36. Wu, C.Y.; Liu, Y.Z. Hyperbranched polysiloxane with highly constrained rings and the effect of the attached arms on the assembly behavior. Polym. Chem. 2017, 8, 6490-6495. [CrossRef]

37. Rubinsztajn, S.; Cella, J.A. A new polycondensation process for the preparation of polysiloxane copolymers. Macromolecules 2005, 38, 1061-1063. [CrossRef]

38. Yu, J.Y.; Liu, Y.Z. Cyclic polysiloxanes with linked cyclotetrasiloxane subunits. Angew. Chem. Int. Ed. 2017, 56, 8706-8871. [CrossRef] [PubMed]

39. Morgan, J.; Chen, T.; Hayes, R.; Dickie, T.; Urlicha, T.; Brook, M.A. Facile synthesis of dendron branched silicone polymers. Polym. Chem. 2017, 8, 2743-2746. [CrossRef]

40. Park, S.H.; Liu, H.M.; Kleinsorge, M.; Grey, C.P.; Toby, B.H.; Parise, J.B. [Li-Si-O]-MFI: A new microporous lithosilicate with the MFI topology. Chem. Mater. 2004, 16, 2605-2614. [CrossRef]

41. Mintcheva, N.; Tanabe, M.; Osakada, K.; Georgieva, I.; Mihailov, T.; Trendafilova, N. Synthesis and characterization of a dinuclear platinum complex with silsesquioxanate ligand. J. Organomet. Chem. 2010, 695, 1738-1743. [CrossRef]

42. Malinovskii, S.T.; Tesuro Vallina, A.; Stoeckli-Evans, H. X-ray diffraction investigation of siloxanes. III. Structure and configuration of cyclic tetra- and pentasiloxanes bearing different organic substituents at silicon atoms. J. Struct. Chem. 2007, 48, 128-136. [CrossRef]

43. Bassindale, A.R.; Liu, Z.H.; Parker, D.J.; Taylor, P.G.; Horton, P.N.; Hursthouse, M.B.; Light, M.E. The reactions of dialkyl and diarylethoxysilanes with $\mathrm{T} 6$ silsesquioxane cages: X-ray crystallographic studies of the mono-T6D1 and bis-T6D2 insertion ring expansion products. J. Organomet. Chem. 2003, 687, 1-11. [CrossRef]

44. Janaa, R.N.; Mukundab, P.G.; Nandoa, G.B. Thermogravimetric analysis of compatibilized blends of low density polyethylene and poly(dimethyl siloxane) rubber. Polym. Degrad. Stab. 2003, 80, 75-82. [CrossRef]

45. Fina, A.; Tabuani, D.; Carniato, F.; Frache, A.; Boccaleri, E.; Camino, G. Polyhedral oligomeric silsesquioxanes (POSS) thermal degradation. Thermochim. Acta 2006, 440, 36-42. [CrossRef]

46. Blanco, I.; Abate, L.; Bottino, F.A. Mono substituted octaphenyl POSSs: The effects of substituents on thermal properties and solubility. Thermochim. Acta 2017, 655, 117-123. [CrossRef]

47. Chiang, C.L.; Ma, C.C.M. Synthesis, characterization, and properties of novel ladderlike phosphorus-containing polysilsesquioxanes. J. Polym. Sci. Part A Polym. Chem. 2003, 41, 1371-1379. [CrossRef]

48. Li, Z.Q.; Yang, R.J. Flame retardancy, thermal and mechanical properties of sulfonate-containing polyhedral oligomeric silsesquioxane (S-POSS)/polycarbonate composites. Polym. Degrad. Stab. 2015, 116, 81-87. [CrossRef]

49. Sheen, Y.C.; Lu, C.H.; Huang, C.F.; Kuo, S.W.; Chang, F.C. Synthesis and characterization of amorphous octakis-functionalized polyhedral oligomeric silsesquioxanes for polymer nanocomposites. Polymer 2008, 49, 4017-4024. [CrossRef]

50. Aryabadie, S.; Sadeghi-kiakhani, M.; Arami, M. Antimicrobial and dyeing studies of treated cotton fabrics by prepared chitosan-PAMAM dendrimer/Ag nanoemulsion. Fibers Polym. 2016, 16, 2529-2537. [CrossRef]

51. Gao, L.; Kojima, K.; Nagashima, H. Transition metal nanoparticles stabilized by ammonium salts of hyperbranched polystyrene: Effect of metals on catalysis of the biphasic hydrogenation of alkenes and arenes. Tetrahedron 2015, 71, 6414-6423. [CrossRef]

52. Tang, Q.; Cheng, F.; Lou, X.L.; Liu, H.J.; Chen, Y. Comparative study of thiol-free amphiphilic hyperbranched and linear polymers for the stabilization of large gold nanoparticles in organic solvent. J. Colloid Interface Sci. 2009, 337, 485-491. [CrossRef] [PubMed]

53. Yu, B.; Jiang, X.S.; Yin, J. Responsive hybrid nanosheets of hyperbranched poly(ether amine) as a 2D-platform for metal nanoparticles. Chem. Commun. 2013, 49, 603-605. [CrossRef] [PubMed]

54. Kuo, P.L.; Chen, C.C.; Yuen, S.M. Protection effects of hydrophile-grafted silicone copolymers on the formation of colloidal silver nanoparticles. Macromolecules 2009, 42, 4937-4940. [CrossRef] 
55. Shankar, R.; Jangira, B.; Sharmaa, A. A novel synthetic approach to poly(hydrosiloxane)s via hydrolytic oxidation of primary organosilanes with a AuNPs-stabilized Pickering interfacial catalyst. RSC Adv. 2017, 7, 344-351. [CrossRef]

56. Stochma, E.; Strzezik, J.; Krowiak, A. Preparation and characterization of polysiloxane networks containing metallic platinum particles. J. Appl. Polym. Sci. 2016, 133, 43096. [CrossRef]

(C) 2018 by the authors. Licensee MDPI, Basel, Switzerland. This article is an open access article distributed under the terms and conditions of the Creative Commons Attribution (CC BY) license (http:/ / creativecommons.org/licenses/by/4.0/). 



\section{MDPI}

St. Alban-Anlage 66

4052 Basel

Switzerland

Tel. +41616837734

Fax +41 613028918

www.mdpi.com

Polymers Editorial Office

E-mail: polymers@mdpi.com

www.mdpi.com/journal/polymers

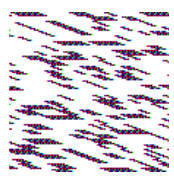



MDPI

St. Alban-Anlage 66

4052 Basel

Switzerland

Tel: +41 616837734

Fax: +41 613028918 